Illinois State University

ISU ReD: Research and eData

Theses and Dissertations

2-16-2021

\title{
An Investigation Of Pre-Service Elementary-School Teachers' Knowledge Of Properties Of Real Numbers
}

Okemwa Ogega Monandi

Illinois State University, oomonandi1@aacc.edu

Follow this and additional works at: https://ir.library.illinoisstate.edu/etd

Part of the Science and Mathematics Education Commons

\section{Recommended Citation}

Monandi, Okemwa Ogega, "An Investigation Of Pre-Service Elementary-School Teachers' Knowledge Of Properties Of Real Numbers" (2021). Theses and Dissertations. 1393.

https://ir.library.illinoisstate.edu/etd/1393

This Dissertation is brought to you for free and open access by ISU ReD: Research and eData. It has been accepted for inclusion in Theses and Dissertations by an authorized administrator of ISU ReD: Research and eData. For more information, please contact ISUReD@ilstu.edu. 


\section{AN INVESTIGATION OF PRE-SERVICE ELEMENTARY-SCHOOL TEACHERS' \\ KNOWLEDGE OF PROPERTIES OF REAL NUMBERS}

\section{OKEMWA OGEGA MONANDI}

237 Pages

Current recommendations for school mathematics include teaching algebra as a $\mathrm{K}-12$ content stranding beginning in the elementary-school grades. The focus of this study was on preservice elementary mathematics teachers' knowledge of algebra content in the intended curriculum for K-8 mathematics. In the Common Core State Standards for Mathematics (CCSSM), great emphasis is placed on numerical structure, and the usefulness of number properties in algebraic manipulation. In this study, preservice teachers' knowledge of structural properties was explored. The purpose of this study was to investigate the knowledge that preservice elementary teachers have about number properties and whether they could apply these properties in meaningful ways. Preservice elementary teachers (PETs) at a community college in the U.S. Mid-Atlantic were sought to participate in this study. Participants were sought from three courses: MT1, MT2, and MT3. The teaching intervention was situated in the MT1 course because part of the course's learning outcomes included fostering numerical and algebraic reasoning. Participating PETs in MT2 and MT3 received no special instruction on properties of real numbers because that content was supposed to have been taught and learned in the MT1 course. Both the quantitative and qualitative analyses revealed that the participating PETs did not demonstrate strong knowledge of properties. The analyses revealed that there was not an educationally significant difference between MT1 and MT2/MT3 participants' knowledge of number of properties. The analyses also revealed that the instructional intervention emphasizing 
properties resulted in modest gains in MT1 PETs' knowledge of properties but the gains were not educationally significant. The findings revealed the challenges of preparing teachers with well-developed structure sense and strong understanding of properties — knowledge that is essential in helping young learners gain relational understandings of early algebra.

KEYWORDS: Early Algebra, Number Properties, Numerical Structure, Preservice Elementary Teachers, Structural Properties, Teacher Preparation 


\title{
AN INVESTIGATION OF PRE-SERVICE ELEMENTARY-SCHOOL TEACHERS' \\ KNOWLEDGE OF PROPERTIES OF REAL NUMBERS
}

OKEMWA OGEGA MONANDI

\author{
A Dissertation Submitted in Partial \\ Fulfillment of the Requirements \\ for the Degree of \\ DOCTOR OF PHILOSOPHY \\ Department of Mathematics \\ ILLINOIS STATE UNIVERSITY
}


(C) 2021 Okemwa Ogega Monandi 


\section{AN INVESTIGATION OF PRE-SERVICE ELEMENTARY-SCHOOL TEACHERS' \\ KNOWLEDGE OF PROPERTIES OF REAL NUMBERS}

OKEMWA OGEGA MONANDI

COMMITTEE MEMBERS:

Edward Mooney, Chair

McKenzie A. Clements

David Barker

Matthew Winsor

John K. Rugutt 


\section{ACKNOWLEDGMENTS}

There are many people who contributed to my education journey that I would like to thank. First, I would like to thank my parents, David Ogega Nyaberi and Norah Nyaboke Mauncho, for their support and encouragement throughout my educational journey. I would like to thank my primary school teachers of mathematics, Mr. Nyaata, Mr. Nyabicha, and Mr. Nyabochwa, for inspiring a lifelong love for mathematics.

I would like to thank Dr. Peter McCarthy and Prof. Yusuf Yildirim for their mentorship in mathematics during my undergraduate years. I would like to thank Dr. Edward Mooney, Dr. John Rugutt, Dr. Ken Clements and Dr. Nerida Ellerton for their guidance, mentorship, and support throughout my doctoral journey. I would like to express gratitude to Dr. David Barker and Dr. Matthew Winsor, member of my dissertation committee, for their assistance and guidance as I worked on my dissertation.

Most importantly, I want to thank my wife, Alberta McCarthy, for her love and patience and encouragement throughout my graduate education journey, for being there through the most difficult of times, and for pushing me to finish my Ph.D. Lastly, I want to thank my son, Samuel Mauncho Monandi, for being my inspiration to seeing anything that I do, especially my Ph.D. studies, to completion.

O. O. M. 


\section{CONTENTS}

Page

ACKNOWLEDGMENTS

TABLES vii

FIGURES Niii

CHAPTER I: INTRODUCTION AND BASIS FOR STUDY 1

Early Algebra

Structure Sense $\quad 2$

The Basis for the Dissertation Study $\quad 4$

First Preliminary Study

$\begin{array}{ll}\text { Second Preliminary Study } & 7\end{array}$

$\begin{array}{ll}\text { Purpose of the Study } & 8\end{array}$

$\begin{array}{ll}\text { Rationale } & 9\end{array}$

Summary of the Present Study 11

$\begin{array}{ll}\text { Research Questions } & 12\end{array}$

CHAPTER II: LITERATURE REVIEW 14

$\begin{array}{ll}\text { Review of Literature } & 14\end{array}$

Importance of Structure in the Early Grades 16

$\begin{array}{ll}\text { Research on Understanding of Structure } & 18\end{array}$

$\begin{array}{ll}\text { Commutative Properties } & 20\end{array}$

$\begin{array}{ll}\text { Associative Properties } & 21\end{array}$

Distributive Property 23

Pre-Service Teachers' Knowledge of Numerical Properties 24 
Theoretical Perspectives

Charles Sanders Peirce and Ferdinand de Saussure 28

$\begin{array}{ll}\text { Semiotic Aspects of Early Algebra } & 29\end{array}$

Semiotic Aspect of Properties of Real Numbers 30

Cognitive Structures Involved in Learning 32

$\begin{array}{ll}\text { Summary } & 37\end{array}$

$\begin{array}{ll}\text { CHAPTER III: RESEARCH METHODS } & 38\end{array}$

$\begin{array}{ll}\text { Setting up for the Study } & 38\end{array}$

$\begin{array}{ll}\text { Mathematics courses for PETs } & 39\end{array}$

$\begin{array}{ll}\text { Teaching Aspect of Study } & 40\end{array}$

Challenges to implementing an instructional intervention 41

Instructional intervention in the MT1 course $\quad 42$

Participants, Instrumentation, and Procedures 48

$\begin{array}{ll}\text { Participants } & 48\end{array}$

$\begin{array}{ll}\text { Instrumentation } & 49\end{array}$

$\begin{array}{lr}\text { Paper-and-pencil assessment instruments } & 49\end{array}$

Audio-taped interviews $\quad 53$

Data Collection Procedures $\quad 55$

$\begin{array}{ll}\text { Methods for Data Analysis } & 57\end{array}$

$\begin{array}{ll}\text { Qualitative Analysis } & 57\end{array}$

$\begin{array}{ll}\text { Quantitative Analysis } & 61\end{array}$

$\begin{array}{ll}\text { Summary } & 67\end{array}$

$\begin{array}{ll}\text { CHAPTER IV: RESULTS } & 68\end{array}$ 
Qualitative Results

MT1 Participants’ Knowledge of Properties 70

$\begin{array}{ll}\text { Commutative property for addition } & 70\end{array}$

$\begin{array}{ll}\text { Commutative property for multiplication } & 72\end{array}$

$\begin{array}{ll}\text { Associative property for addition } & 73\end{array}$

$\begin{array}{ll}\text { Associative property for multiplication } & 75\end{array}$

$\begin{array}{ll}\text { Distributive property } & 76\end{array}$

MT2/MT3 Participants' Knowledge of Properties $\quad 79$

$\begin{array}{ll}\text { Commutative property for addition } & 79\end{array}$

$\begin{array}{ll}\text { Commutative property for multiplication } & 80\end{array}$

$\begin{array}{ll}\text { Associative property for addition } & 81\end{array}$

Associative property for multiplication $\quad 82$

$\begin{array}{ll}\text { Distributive property } & 83\end{array}$

Other Questions on the Paper-and-Pencil Instruments 86

$\begin{array}{lr}\text { Interview Data Findings } & 90\end{array}$

$\begin{array}{ll}\text { MT1 PETs (Ally and Betty) } & 90\end{array}$

$\begin{array}{ll}\text { Knowledge of the distributive property } & 90\end{array}$

Knowledge of the commutative property for addition $\quad 92$

Knowledge of the commutative property for multiplication 93

$\begin{array}{ll}\text { Knowledge of the associative property for addition } & 94\end{array}$

Knowledge of the associative property for multiplication 96

$\begin{array}{ll}\text { Knowledge of inverse and identity properties } & 97\end{array}$

$\begin{array}{lr}\text { MT2/MT3 PETs (Casey and Daisy) } & 98\end{array}$ 
$\begin{array}{ll}\text { Knowledge of the distributive property } & 98\end{array}$

Knowledge of the commutative property for addition 99

Knowledge of the commutative property for multiplication 99

$\begin{array}{ll}\text { Knowledge of the associative property for addition } & 100\end{array}$

$\begin{array}{ll}\text { Knowledge of the associative property for multiplication } & 101\end{array}$

$\begin{array}{ll}\text { Knowledge of inverse and identity properties } & 101\end{array}$

$\begin{array}{ll}\text { Quantitative Results } & 102\end{array}$

Pre-Teaching vs. Post-Teaching Performance of MT1 PETs 103

Cohen's $d$ effect sizes and Performance Improvement Ratios 106

$\begin{array}{ll}\text { MT1 vs. MT2/MT3 Performance } & 108\end{array}$

Comparing MT1 pre-teaching and MT2/MT3 performances 109

Comparing MT1 post-teaching and MT2/MT3 performances $\quad 111$

Summary and Concluding Comments 112

CHAPTER V: DISCUSSION AND CONCLUDING REMARKS 116

Answers to Research Question 1

Answer to Question 1a-PETs’ Knowledge of Commutative Properties 117

Answer to Question 1b_PETs' Knowledge of Associative Properties 118

Answer to Question 1c_-PETs' Knowledge of the Distributive Property 119

Answer to Question 1d_-PETs’ Knowledge of Inverse and Identity Properties $\quad 120$

Answers to Research Question 2

$\begin{array}{ll}\text { Answer to Research Question } 3 & 127\end{array}$

$\begin{array}{ll}\text { Concluding Remarks } & 129\end{array}$

$\begin{array}{ll}\text { Limitations of the Study } & 131\end{array}$ 
Implications

Reflecting on the Teaching Intervention

Implications for Teaching

Implications for Future Interventions

Recommendations for Future Research

APPENDIX A: NUMBER PROPERTIES IN THE COMMON CORE STANDARDS

APPENDIX C: PAPER-AND-PENCIL ASSESSMENTS (TWO PARALLEL VERSIONS) 163

APPENDIX D: CHARTS FOR PROPERTIES OF WHOLE-NUMBER OPERATIONS 


\section{TABLES}

Table

Page

1. NPQ Number Sentences and Corresponding Property

2. Nine Questions on Using Number Properties

3. Pre-T and Post-T Responses to Commutative Property Questions

4. Pre-T and Post-T Responses to Associative Property Questions

5. Pre-T and Post-T Responses to Distributive Property Questions

6. MT2/MT3 Responses to Commutative Property Questions

7. MT2/MT3 Responses to Associative Property Questions

8. MT2/MT3 Responses to Distributive Property Questions

9. Cohen's $d$ effect sizes and PIRs for MT1 pre- and post-teaching scores

10. Results of Independent $t$-tests and Descriptive Statistics MT1 Pre-Teaching vs MT2/MT3

11. Cohen's $d$ effect sizes and $r^{2}$ for MT1 pre-teaching scores vs MT2/MT3 scores

12. Results of Independent $t$-tests and Descriptive Statistics MT1 Post-Teaching vs MT2/MT3

13. Cohen's $d$ effect sizes and $r^{2}$ for MT1 post-teaching scores vs MT2/MT3 scores 


\section{FIGURES}

Figure $\quad$ Page

1. MT1 Course Schedule 42

2. Sample of Participant's Response to Question 2

3. Sample of Participant's Response to Question $3 \quad 60$

4. Change in a Student's Concept Image of the Commutative Property for Multiplication 72

5. A Sample Response to Q14 and Q15 from One of the MT3 Participants

6. Skip-count Models for $5 \times 3$ and $3 \times 5$ 


\section{CHAPTER I: INTRODUCTION AND BASIS FOR STUDY}

For many years, researchers in mathematics education have identified school algebra as a problematic subject-matter for students across all grades including the post-secondary school years (e.g., Booth, 1988; Hart, 1981; Kanbir, Clements, \& Ellerton, 2018; Kieran, 1992, 2007; Welder, 2012). Kieran (1992, 2007) attributed students' challenges in algebra to inadequate understanding of arithmetic. Blanton and Kaput $(2005,2011)$ comparably asserted that students' difficulties could be connected to their inability to transfer fluently from arithmetic to algebra. Kanbir, Clements, and Ellerton (2018) proposed that students' issues with transition from arithmetic to algebra could be attributed to their lack of understanding of structure in numerical aspects of school mathematics.

\section{Early Algebra}

The traditional route of school mathematics involves introducing arithmetic in the elementary school and reserving formal algebra for secondary school (Blanton \& Kaput, 2005, 2011). Maria Blanton and her colleagues (Blanton \& Kaput, 2005, 2011; Blanton et al., 2015; Kaput, 2008; Kaput, Carraher, \& Blanton, 2008) have asserted that school algebra should be approached as a K-12 content strand whereby students are introduced to algebra through ageappropriate algebraic activities in the elementary-school grades and receive continued learning experiences in algebra through their middle-school and secondary-school education. Blanton and Kaput $(2005,2011)$ and Blanton et al. (2015) reported that young children were capable of reasoning and thinking algebraically and supported the inclusion of algebra in the elementary and middle-school grades.

The term early algebra has been used to characterize the elevated focus on algebraic thinking and algebraic concepts in the elementary and middle-school grades. Carraher and 
Schliemann (2007) described early algebra as encompassing "algebraic reasoning and algebrarelated instruction among young learners-from approximately 6 to 12 years of age" (p. 670). Carraher and Schliemann (2007) emphasized that early algebra did not mean introducing the formal algebra that is traditionally introduced in the eighth or ninth grade to elementary-school students. Instead, they emphasized that the mathematics content that is traditionally taught to young learners situated in the domain of arithmetic has an inherently algebraic character that can be exploited to help young learners develop their burgeoning conceptualization of algebra.

Kanbir et al. (2018) contended that special attention should be given to properties of real numbers as part of early algebra instruction because of the important role that these properties have in formal algebra. Howe (2005) wrote that the properties of real numbers form the basis for legal manipulations of expressions in algebra, and these properties, "together with the principles for transforming equations ... summarize the basis for algebraic technique" (p. 1). Accordingly, early algebra instruction should provide young learners of mathematics opportunities to, interact with, and develop their understanding of these properties when operating on numbers (Kanbir et al., 2018; Kaput, 2008; Ma, 1999; Schifter, Monk, Russell, \& Bastable, 2008; Usiskin, 1988).

\section{Structure Sense}

In the context of early algebra, structure sense refers to one's capacity to apply properties of real numbers to manipulate mathematical expressions so that they be readily simplified, evaluated, or interpreted (Kanbir, 2016; Kanbir, Clements, \& Ellerton, 2018; Hoch \& Dreyfus, 2004; Novotna \& Hoch, 2008). Hoch and Dreyfus (2004) described structure sense as the ability to recognize that a mathematical expression is a structural entity that can be manipulated without changing its internal meaning and to recognize which manipulations are legal and valid and which are useful. Kanbir et al. (2018) wrote that manipulations of algebraic expressions relied on 
proper applications of properties of real numbers. Accordingly, proper application of number properties in the manipulation of mathematical expressions require an understanding of structure in mathematics.

To demonstrate further the meaning of structure sense, I discuss different approaches to simplifying a numerical expression that were discussed in the Kanbir et al. (2018) study. In their study, Kanbir et al. asked students to find the value of $4 \times\left(\frac{1}{4} \times 128\right)$ without using a calculator. A student using structure sense would evaluate this numerical expression by associating 4 and $\frac{1}{4}$. A student that recognizes that 4 and $\frac{1}{4}$ can be associated is cognizant of the underlying structure of the expression. Hoch and Dreyfus (2004) would assert that such a student is cognizant of the internal order of the expression because the student sees that $\left(4 \times \frac{1}{4}\right) \times 128$ is equivalent to $4 \times\left(\frac{1}{4} \times 128\right)$ by the associative property for multiplication. By first finding the value of $4 \times \frac{1}{4}$ and multiplying that value by 128 , a student using structure sense recognizes that this approach is more accessible and more useful in this problem situation. However, a student that evaluates the given numerical expression by relying on the mnemonic PEMDAS (Parenthesis, Exponent, Multiplication, Division, Addition, and Subtraction) is not using structure sense. Such as a student would first find the value of $\frac{1}{4} \times 128$ because this part is situated within the parenthesis, and then multiply the resulting value by 4 . It is likely that such a student is not aware of the underlying structure of the numerical expression and is just mechanically applying the mnemonic PEMDAS for order of operations.

The authors of the Common Core State Standards for Mathematics (CCSSM), published by the National Governors Association Center for Best Practices (NGA) and the Council of Chief State School Officers (CCSSO) in 2010, expect students to know and be able to use 
number properties in simplifying and interpreting numerical expressions by the time they begin their secondary-school mathematics studies. The stated expectations listed under algebra for grades 3-5 in the Principles and Standards for School Mathematics (National Council of Teachers of Mathematics [NCTM], 2000) assert that all students are expected to identify the properties of "commutativity, associativity, and distributivity and use them to compute with whole numbers" (p. 158). The goal of this dissertation study was to investigate the knowledge that preservice elementary teachers had about number properties and whether they could apply these properties in meaningful ways - competence expected of students in the middle-school grades.

\section{The Basis for the Dissertation Study}

Kanbir and colleagues' (Kanbir, 2016; Kanbir et al., 2018) research served as the motivation for conducting this dissertation study. Part of their research included examining seventh-graders' knowledge of, and ability to apply, the associate properties for addition and multiplication and the distributive property for multiplication over addition in solving, simplifying, evaluating and interpreting mathematical expressions and equations. According to the CCSSM (2010) and NCTM (2000), students are expected to have developed this competence by the time they enter the seventh grade. Kanbir and colleagues' research revealed that the seventh-grade student participants in their studies did not have adequate knowledge of any of three number properties that were of interest to the researchers (the associate property for addition and multiplication and the distributive property); nor could the student participants apply these properties in useful ways to solve, simplify, or interpret mathematical expressions and equations. Kanbir et al. (2018) speculated that the lack of knowledge of number properties 
which was observed among seventh-grade students would also be observed among beginning teachers of algebra.

In two preliminary studies (Monandi, 2018, 2019), I sought to examine the veracity of Kanbir et al's conjecture. My search for literature regarding preservice teachers' knowledge of number properties yielded scant results. Few studies have been conducted investigating elementary or middle-school teachers' knowledge of properties of real numbers. The scarcity of literature regarding this matter, in part, motivated me to proceed with my preliminary studies. The two preliminary studies revealed that preservice teachers did not have strong knowledge and understanding of properties of real numbers and of how to apply these properties in meaningful ways to solve and simplify algebraic problems. Meixia Ding and her associates (see e.g., Barnett

\& Ding, 2019; Ding, 2016; Ding, Li, \& Capraro, 2013) and Kyungsoon Jeon (2012) are among the few researchers who have studied aspects of preservice teachers' knowledge of number properties, and their findings support those of my preliminary studies. Ding, Li and Capraro (2013) reported that most of the preservice teachers in their study did not have sufficient knowledge of the meaning of the associative property for multiplication and could not use this property in meaningful ways. Jeon (2012) found that many of her preservice teachers knew the mnemonic for PEMDAS but did not have a thorough understanding of when and when not to use it.

\section{First Preliminary Study}

In the first preliminary study (Monandi, 2018), I investigated preservice elementary teachers' knowledge of number properties, specifically the associative property for addition and for multiplication and the distributive property for multiplication over addition, and their knowledge of explicit and recursive representations of numerical patterns. Because the focus of 
this dissertation study is related to the application of properties of real numbers, I will discuss findings from the first preliminary study that pertain to that aspect.

Findings from the first preliminary study revealed that most of the preservice teacher participants were not able to apply number properties in meaningful ways to solve and simplify mathematical expressions. The following three questions were among those that were assessed on the pre-teaching assessment:

1. Suppose you were asked to calculate the value of $940+(60+403)$ in your head (without writing anything down or using a calculator). How would you do it, and which property would you be using?

2. What would be a quick method of finding the value of $7 \times 97+7 \times 3$ without using a calculator? What is the property which allows you to use that quick method?

3. What would be a quick method of finding the value of $64 \times\left(\frac{1}{32} \times 120\right)$, without using a calculator?

The first question (Q1) was intended to assess whether the participating preservice elementary teachers (PETs) could recognize that the numerical expression in this question could be readily simplified by applying the associative property for addition and whether they could name the property. At the pre-teaching stage, only 5 of the 17 participating PETs were able to correctly answer this question by applying the associative property for. Of these 5 , only 2 correctly named the property.

The second question (Q2) was intended to assess whether the participating PETs could recognize that the numerical expression in $\mathrm{Q} 2$ could be readily simplified by applying the distributive property for multiplication over addition and whether the participating PETs could 
name this property. At the pre-teaching stage, none of the 17 participating preservice teachers were able to answer this question correctly by applying the distributive property.

The third question (Q3) was intended to assess whether participating PETs could recognize that the numerical expression in Q3 could be readily simplified by applying the associative property for multiplication. At the pre-teaching stage, only 6 of the 17 participating preservice teachers were able to answer this question correctly by applying the associative property for multiplication.

\section{Second Preliminary Study}

In the second preliminary study (Monandi, 2019), I investigated whether preservice middle-school teachers' exposure to properties of groups and fields influenced their perceptions of properties of real numbers and the relevance of these properties in the middle-school mathematics curricula. The participating preservice middle-school teachers completed two assessments prior to instruction; one of them was on number sense and the other on structure sense. The pre-teaching structure-sense test had the same questions has those utilized on the first preliminary study. So, the three sample questions provided in the preceding section were also on the structure-sense test that the participating preservice middle-school teachers (PMTs) took at the pre-teaching stage.

On the pretest, 8 of the 14 participating PMTs answered Q1 correctly by applying the associative property for addition. However, only 1 of these 8 correctly named the property. On Q2, 10 of the 14 participating PMTs answered the question correctly but only 2 of them identified the distributive property by name. For Q3, 6 of the 14 participating PMTs answered the question correctly. Many preservice teacher participants in both preliminary studies erroneously named the associative property for addition as the commutative property or as the 
commutative property for addition. In the secondary preliminary study, some of the participating PMTs erroneously named that property that allowed them readily to simplify the numerical expression in Q2 as the factoring property. The results from both preliminary studies indicated that the participating preservice teachers did not have strong knowledge of properties of real numbers. Some of them intuitively applied number properties without correctly identifying the properties by name.

\section{Purpose of the Study}

The purpose of this dissertation study was to extend beyond the scope of these preliminary studies and conduct a more comprehensive study on the aspect of structure in school mathematics. Both preliminary studies addressed this aspect, but each study had other aspects that were emphasized. The first preliminary study was focused not only on the structure aspect but also on the patterning and modeling aspect. The second preliminary study was focused on whether exposing preservice middle-school teachers to the axiomatic properties of groups and fields helped develop greater understanding of field properties of real numbers.

The focus of this dissertation study was on preservice elementary-school teachers' structure sense as it relates to early algebra. The scope of investigation included examining what preservice elementary-school teachers (hereafter "PETs") knew about each of the following properties of real numbers: the associative property for addition, the associative for multiplication, the commutative property for addition, the commutative property for multiplication, the distributive property for multiplication over addition, the additive identity, the multiplicative identity, the additive inverse, and the multiplicative inverse. The goal was to investigate the extent of PETs' knowledge of, and capacity to apply, properties of real numbers in meaningful way to solve or simplify algebraic expressions. 


\section{Rationale}

This study was important and necessary because it is concerned with what prospective elementary-school teachers know and understand about properties of real numbers. The CCSSM (2010) and NCTM (2000) documents recommended that young learners be introduced to the numerical aspects of structure in the elementary and middle-school grades. This includes helping them identify the properties of "commutativity, associativity, and distributivity and use them to compute with whole numbers" (NCTM, 2010, p. 158). The CCSSM (2010) authors considered number properties so important that they included a glossary listing all the relevant number properties (see CCSSM, 2010, p. 90). Some researchers (see e.g., Kanbir, 2016; Kanbir et al., 2018; Kaput, 2008; Ma, 1999; Schifter et al., 2008) have supported this recommendation emphasizing structure in numerical aspects of school mathematics beginning in the elementaryschool grades. In this study, I investigated what prospective teachers knew and understood about numerical aspects of structure in school mathematics.

Kanbir (2016) and Kanbir et al. (2018) called to attention that the focus of the majority of early algebra research was on the functional thinking and modeling aspects of school mathematics. Many researchers (see e.g., Blanton et al., 2015; Blanton \& Kaput, 2011; Cai \& Knuth, 2011; Radford, 2006, 2011) have a preference for the idea that young learners of mathematics ought to be, and can be, successfully introduced to algebra through the modeling and functional thinking approach. The modeling aspect of school mathematics is evident across all grades in the CCSSM (2010) document. According to the CCSSM (2010) document, grade 3 students are expected to learn to "solve problems involving the four operations and identify and explain patterns in arithmetic" (p. 23); grade 4 students are expected to learn to generate and analyze patterns and to describe them both explicitly and recursively; and grade 5 students are 
expected to learn to analyze patterns and relationships and to generate a numerical pattern given a rule.

Kanbir (2016) and Kanbir et al. (2018) found that introducing students to algebra through the modeling and functional thinking approach did not support their knowledge of the structural aspects of algebra. Likewise, learning the structural aspects of algebra did not support the modeling and functional thinking aspect. Kanbir (2016) and Kanbir et al. (2018) recommended that young mathematics learners should be afforded learning opportunities that emphasize both the structural and modeling aspects of algebra. This means teachers of young mathematics learners should be provided appropriate educational opportunities so they may learn and understand both the structural and modeling aspects of algebra. This study is concerned with prospective teachers' understanding of the structural aspect; the structural aspect has received lesser attention in the literature compared to the modeling aspect (Kanbir, 2016; Kanbir et al., 2018). Ma (1999) contended that effective elementary-school teachers needed to have strong understandings of structure in elementary mathematics. If we accept Ma's claim, then teacher preparation programs ought to emphasize knowledge of the structural side of algebra. This means the numerical aspects of structure should be given a significant level of focus in mathematics teacher preparation. This study contributes to the body of research on preparing elementary-school teachers and on literature of the importance of preparing teachers that can support a young learner's burgeoning understanding of structure.

Rote learning of the mnemonic PEMDAS for order of operations has shown to be a hindrance on a young learner's burgeoning understanding of structure in mathematics (Dupree, 2016; Kanbir, 2016. Kanbir et al., 2018; Lee, Licwinko, \& Taylor-Buckner, 2013). Although young students can remember that the mnemonic references "parentheses, exponents, 
multiplication, division, addition, and subtraction," many still obtain incorrect calculations. Young students, and even prospective teachers, fail to correctly use the order of operations when performing numerical calculations (Dupree, 2016; Jeon, 2012). Some of the misconceptions associated with the mnemonic PEMDAS include the idea that multiplication must come before division and that addition must come before subtraction. These misconceptions can persist well beyond the elementary-school grades and continue through the post-secondary years and beyond (Dupree, 2016; Jeon, 2012).

The study of order of operations is important, but rote learning of this concept hinders development of one's numerical structure sense (Dupree, 2016; Jeon, 2012: Kanbir, 2016. Kanbir et al., 2018; Lee at al., 2013). "With rigid memorization of the order of operations demanded by PEMDAS, students can not only get wrong answers for calculations, but also fail to learn important structural principles" (Kanbir, 2016, p. 43). Instead of rigid memorization, emphasizing the connections among arithmetic operations would provide students the opportunity "to understand PEMDAS with mathematical reasoning" (Lee at al., 2013, p. 74). Using order of operations is not always the best strategy when evaluating numerical expressions. Having numerical structure sense requires knowing when it is appropriate to use order of operations and when it is appropriate to use properties of operations (Barnett \& Ding, 2019;

Kanbir, 2016; Kanbir et al., 2018). In this study, I investigated prospective teachers' numerical structure sense. I studied whether prospective teachers recognized opportunities when using properties of operations to compute with numbers superseded using order of operations.

\section{Summary of the Present Study}

PETs from three mathematics content courses for elementary school teachers agreed to participate in this dissertation study. The three courses are typically taken in succession. The 
decision to have participants from three different mathematics courses for teachers was made because I wanted to compare PETs' knowledge of properties, at different stages of course completion. The first mathematics course for PETs (hereafter- "MT1") is focused on problemsolving techniques, decimal and non-decimal numeral systems, mental computations, estimation, set theory, numerical reasoning, and algebraic reasoning. The second mathematics course (hereafter- "MT2") is focused on investigating measurement, two- and three-dimensional shapes, and geometry concepts. The third mathematics course (hereafter- "MT3") is focused on data collection, data interpretation, sampling, correlation, regression, distributions, and probability.

Participating PETs in the MT1 course participated in lessons that emphasized properties of real numbers and completed pre-teaching and post-teaching assessments. Two MT1 participants also agreed to participate in pre-teaching and post-teaching audio-taped interviews. Participating PETs in MT2 and MT3 received no special instruction on properties of real numbers because that content was supposed to have been taught and learned in the MT1 course. Because there were no specialized lessons on properties, MT2 and MT3 participants were asked to complete only one single assessment. One MT2 participant and one MT3 participant consented to participating in audio-taped interviews.

\section{Research Questions}

In this dissertation study I investigated participating PETs' knowledge of, and capacity to apply, properties of real of numbers in solving and simplifying algebraic problems. The following research questions were used to guide my study:

1) What knowledge do PETs have of number properties? 
a. What knowledge of the commutative property for addition/multiplication do PET demonstrate?

b. What knowledge of the associative property for addition/multiplication do PETs demonstrate?

c. What knowledge of the distributive property do PETs demonstrate?

d. What knowledge of inverse and identity properties do participating PETs demonstrate?

2) How does the PETs' understanding of number properties prior to a teaching intervention compare to the PETs' understanding of number properties after that teaching intervention?

3) How does the understanding of number properties of PETs in a course focused on number properties compare to the understanding of number properties of PETs who previously took the course? 


\section{CHAPTER II: LITERATURE REVIEW}

In this chapter, the literature related to structure sense in the context of $\mathrm{K}-8$ school algebra is reviewed and discussed. The theoretical perspectives that influenced this study are also presented and discussed in this chapter. This dissertation study is an investigation of pre-service teachers' understanding on properties of real numbers. Properties of real numbers play a fundamental role in arithmetic operations and in algebra and beyond (Barnett \& Ding, 2019; Ding, 2016; Ding et al., 2013; Kanbir, 2016; Kanbir et al., 2018; Ma, 1999). In the extant literature, properties of real numbers are also referred to as properties of operations, arithmetic properties, arithmetic principles, number properties, structural properties or as axiomatic properties. Throughout the literature review, these terminologies which refer to properties of real numbers are used.

\section{Review of Literature}

Mason, Stephens, and Watson (2009) asserted that helping learners of mathematics understand and appreciate structure in mathematics was an important component of mathematics teaching and learning. They posited that students should be provided with opportunities to interact with "mathematical structure, at every age and stage of their exposure to mathematics and to mathematical thinking" (Mason, Stephens, \& Watson, 2009, p. 11). The importance of mathematical structure to mathematical understanding has also been put forward by other researchers including Hoch and Dreyfus (2004), Kanbir, Clements, and Ellerton (2018), Ma (1999), and Novotna and Hoch (2008).

Mason et al. (2009) defined mathematical structure as the identification of general properties which are derivative of, or satisfied under, certain conditions such as those that arise in relationships between elements. 
These elements can be mathematical objects like numbers and triangles, sets with functions between them, relations on sets, even relations between relations in an ongoing hierarchy. Usually it is helpful to think of structure in terms of an agreed list of properties which are taken as axioms and from which other properties can be deduced. (Mason et al., 2009, p. 10)

Mason et al. noted that each area of mathematics has some set of axiomatic properties which form the basis for reasoning and operating within that mathematical area. For example, they noted that Euclidean plane geometry is predicated on axioms and postulates which are accepted as the foundational basis for reasoning and deduction in that area of mathematics.

For Hoch and Dreyfus (2004) and Novotna and Hoch (2008), the term structure could be understood and interpreted differently because it has contextual meaning. They noted that in the realm of abstract algebra, for example, structure could refer to the properties which arise, or are derived, when a set of elements is combined under one or more operations. However, they noted that in the context of school algebra, structure could refer to the manner in which properties of real numbers can be applied in algebraic reasoning. Hoch and colleagues' (Hoch \& Dreyfus, 2004; Novotna \& Hoch, 2008) definition of structure is comparable to that of Mason et al. (2009). Both definitions are concerned with the identification and significance of axiomatic properties in mathematics.

This study is concerned with what prospective elementary-school teachers know and understand about properties of real numbers and their capacity to apply these properties when computing with real numbers or when evaluating or simplifying numerical expressions. Those who prepared the Standards documents (NCTM, 2000; NGA \& CCSSO, 2010) and some mathematics education researchers (e.g., Kanbir et al., 2018; Ma, 1999; Mason et al., 2009) have 
emphasized the importance of helping young learners understand structure in mathematics. Properties of real numbers are considered fundamental ideas in mathematics (Barnett \& Ding, 2019; Kanbir et al., 2018; Ma, 1999), and they are greatly emphasized in Standards documents across the early grades (NCTM, 2000; NGA \& CCSSO, 2010) because they form the basis for arithmetic computation strategies (Barnett \& Ding, 2019; Schifter et al., 2008). CCSSM (2010) content statements containing language highlighting the pertinence of properties of real numbers in elementary and middle-school curricula are presented Appendix A.

\section{Importance of Structure in the Early Grades}

Researchers in mathematics education have identified school algebra as a problematic subject-matter for students, and have attributed difficulty to students' inadequate understanding of arithmetic and their inability to transfer fluently from arithmetic to algebra (Blanton \& Kaput, 2005, 2011; Kanbir et al., 2018; Kieran, 1992, 2007; Wang, 2015; Welder, 2012). Researchers such as Blanton et al. (2015) have recommended that school algebra be approached as a K-12 content strand whereby school children are provided algebra learning experiences beginning in the elementary-school grades. Kanbir, et al. (2018) attributed students' inability to transfer fluently from arithmetic to algebra to their lack of understanding of structure in mathematics. They contended that early algebra instruction should include learning experiences that help nurture young learners' conceptualization of both the functional thinking and structural aspects of algebra. Kanbir et al. (2018) argued that even though the Standards document emphasize the importance of helping young learners understand structure in mathematics, they argued that many early algebra researchers have displayed a preference for introducing young learners of mathematics to algebra through the functional-thinking approach. However, according to Kanbir 
et al. (2018), a comprehensive treatment of school algebra as a $\mathrm{K}-12$ content strand should also include increased attention to structure in the elementary-school grades.

To alleviate students' issues with the transition from arithmetic to algebra, Barnett and Ding (2019) have also supported the fusion of structure into mathematics instruction in the early grades so to increase students' capability to succeed in later algebra instruction. Extant literature on the teaching of early algebra has progressively accentuated the importance of helping young learners of mathematics make connections between arithmetic operations and their properties (Barnett \& Ding, 2019; Kanbir et al., 2018; Lins \& Kaput, 2004). Helping young learners make connections between arithmetic operations and their properties complements their development of "generalized arithmetic" (Barnett \& Ding, 2019; Blanton et al., 2015; Carraher \& Schliemann, 2007; Stephens, Ellis, Blanton, \& Brizuela, 2017; Trent, 2006). Generalized arithmetic involves "generalizing arithmetic relationships, including fundamental properties of number and operation (e.g., the commutative property of addition), and reasoning about the structure of arithmetic expressions rather than their computational value" (Blanton et al., 2015, p. 43). Nurturing young learners' conceptualization of structure also supports their development of the functional thinking and modeling aspects of algebra (Barnett \& Ding, 2019; Blanton \& Kaput, 2005; Carraher, Schliemann, Brizuela, \& Earnest, 2006; Kanbir, 2016; Kanbir et al., 2018).

Barnett and Ding (2019) emphasized that it was not only important to know how to use properties of real numbers implicitly in computational strategies but it was also important to help "students develop an explicit awareness of the role of these properties in justifying those strategies: They need to learn to 'see' the properties of arithmetic operations as a set of 'rules' which justify mathematical manipulation” (p. 149). This present study examines the extent to which prospective elementary-school teachers know and understand structural aspects of early 
algebra and whether participation in a mathematics content course whereby number properties were emphasized helped prospective teachers improve their knowledge of structure in early algebra. This study is framed from the perspective that understanding mathematical structure is a fundamental aspect of mathematical understanding, and all teachers of mathematics should provide learners of mathematics with opportunities to embrace mathematical structure in their learning of fundamental mathematics.

\section{Research on Understanding of Structure}

In the early grades, young learners are introduced to fact-families - such as $9+5=14,5$ $+9=14,14-5=9$, and $14-9=5$ - to relate some addition or subtraction facts involving the same numbers: 5, 9, and 14 . In the case of multiplication and division, a fact-family of three numbers could be $4 \times 3=12,3 \times 4=12,12 \div 4=3$, and $12 \div 3=4$. This multiplication/division fact-family consists of three numbers: 3,4 , and 12 . Young learners are taught about fact-families as a way for them to memorize addition/subtraction and multiplication/division facts relating to the same three numbers. Learning fact-families is "a simple process of forming an association $\ldots$, such as $7+6 \ldots$ and its answer, $13 \ldots$ This basic process requires neither conceptual understanding nor taking into account a child's developmental readiness" (Baroody, 2006, p. 24).

Learning number facts through rote practice and memorization does not help young learners develop skills for basic arithmetic operations. Instead, understanding of number facts is developed when young learners interact with numerous arithmetic patterns and relationships that interconnect the fact-families (Baroody, 2006). This allows young learners to develop number structure sense, which allows them to understand a number in various forms $(9+1=10,8+2=$ 10 , or $10+0=10$ ) and recognize arithmetic relationships such as $8+4=8+2+2=10+2$. Young learners' burgeoning understanding should be promoted by encouraging them "to focus 
on looking for patterns and relationships; to use these discoveries to construct reasoning strategies; and to share, justify, and discuss their strategies" (Baroody, 2006, p. 29).

Numerical structure sense is not something which is imposed on young learners but is rather developed gradually by helping young learners build up fundamental ideas, such as basic arithmetic properties and the role of properties in arithmetic computations (Barnett \& Ding, 2019; Kanbir et al., 2018). Understanding both the composition and decomposition of numbers and the concept of equality in a number sentence is critical for developing number structure sense, and this plays an important role in later learning of structure in school algebra (Barnett \& Ding, 2019; Baroody, 2006; Kanbir et al., 2018). Research has shown that young learners can make intuitive judgements based on properties when performing arithmetic calculations using invented strategies (Baek, 2008; Barnett \& Ding, 2019; Baroody, 2006; Carpenter, Levi, Berman, \& Pligge, 2005). Baek (2008) found that young learners who especially understood the associative and distributive properties were more successful in evaluating multi-digit multiplication problems.

Numerical structure sense requires understanding of both the composition and decomposition of numbers and the concept of equality in a number sentence. Most extant literatures focus on the meaning of the equal sign and the concept of equality, but few focus on numerical structure sense and the importance of properties of numbers. Kanbir et al. (2018) studied middle-school students' understanding of structure of middle-school numerical properties and algebra. They attributed students' difficulty in algebra to their inadequate understanding of structure and their inability to transfer fluently from arithmetic to algebra. To achieve the transition from arithmetic to algebra ... students should first have the knowledge and skills found in the arithmetic step ... This knowledge includes knowledge 
of fundamental properties of operations, such as commutative property, associative property and distributive property, and the skills include the ability to work backward and to recognize that the values on both sides of an equal sign are the same. (Kose \& Kiziltoprak, 2020, p. 136)

\section{Commutative Properties}

Addition fact-families, such as $9+5=14$ and $5+9=14$, and multiplication factfamilies, such as $4 \times 3=12$ and $3 \times 4=12$, offer opportunities through which teachers can introduce young learners to the commutativity of addition and multiplication operations. Thanheiser, Philipp, Fasteen, Strand, and Mills (2013) suggested that commutative properties, and other properties, are mostly taught procedurally in $\mathrm{K}-8$ schools. The commutative properties, for example, are taught procedurally using informal language such as "switch around" and "turnarounds" because the operands in an addition or multiplication number sentence can be interchanged without changing the sum or product, respectively (Anthony \& Walshaw, 2002; Warren \& English, 2000).

Anthony and Walshaw (2002) found that students in their study could not articulate meaningfully the commutative property for multiplication. The students in their study did not make the connection between the array model and the commutative property for multiplication and could not explain accurately the concept of commutativity. Nor could students connect the ideas of factors and multiples and the concept of commutativity in multiplicative thinking. Warren and English (2000) found that although almost all of the students in their study could identify the commutative properties for addition/multiplication, many of them could not generate additional examples of the commutative properties, and only a handful provided acceptable 
explanations of the property. Warren and English noted that students were inclined to reason procedurally rather than conceptually with regards to understanding of mathematics structure.

To help learners of mathematics understand the concept of commutativity, the commutative properties must not be taught superficially. Superficial understanding of commutativity — knowing only that addition and multiplication are commutative because changing the order of the operands is permissible - is not sufficient. It is also important to understand why interchanging operands does not affect the sum or product. Larsen's (2010) attributed students' difficulties with the associative and commutative properties to their imprecise and informal understanding of these properties.

Teachers need to understand the structure of the mathematics around the commutative property and explicitly use terminology such as "factors and multiples" and the array as a model for understanding the property, rather than allow students to continue to discuss commutativity in terms of "switch arounds" and the like. (Hurst, 2017, p. 4)

\section{Associative Properties}

Arithmetic relationships such as $8+4=8+2+2=10+2$ can be justified using the associative property for addition: $8+4=8+(2+2)=(8+2)+2=10+2=12$. When adding real numbers, the change in grouping of three or more addends does not affect the sum: $(a+b)+$ $c=a+(b+c)$. The associative property for addition can be used to support addition strategies such as decomposing addition sentences into easier/known expressions: $7+8=7+(7+1)=(7$ $+7)+1=14+1=15$ (Baroody, 2006). Multiplication of real numbers is also associative. When multiplying real numbers, the change in grouping of three or more factors does not affect the product: $(a \times b) \times c=a \times(b \times c)$. The associative property for multiplication can be used to support multiplication strategies such as double-halving, $16 \times 50=(8 \times 2) \times 50$. The associative 
property can also be employed to re-write number sentences to ease mental computations, $(8 \times 4)$ $\times 25=8 \times(4 \times 25)=8 \times 100$ (Fauskanger $\&$ Bjuland, 2019). Teachers should help students develop an explicit awareness of the role of these properties in the computation strategies (Barnett \& Ding, 2019)

Students, and PETs alike, often conflate the associative and commutative properties (Ding et al., 2013; Monandi, 2018, 2019). Larson (2010) attributed students' issues with associative and commutative properties to poor foundational knowledge of the meanings and definitions of these properties. Kanbir et al. (2018), who studied seventh-graders knowledge and understanding of the associative properties for addition and for multiplication, reported that at the pre-intervention stage, participating seventh-graders had virtually no knowledge of the associative property for addition or for multiplication. This indicates that intended curricula for early algebra is not occurring effectively in schools as recommended in the Standards documents (NCTM, 2000; NGA \& CCSSO, 2010) for K-8 school mathematics.

Barnett and Ding (2019) investigated the teaching of the associative property in elementary-school classrooms in a large urban school district in the United States. They observed that teachers in most classrooms did not help students develop an explicit awareness of the associative property in relation to computation strategies. They found that in most classrooms the associative property was only formally introduced without contextualized examples to illustrate the meaning of the property. Their findings regarding the teaching of the associative property confirmed Thanheiser et al.'s (2013) suggestion that properties of real numbers in K-8 grades are taught procedurally rather than conceptually. Thus, young learners of mathematics are not afforded opportunities to develop conceptual understandings of properties and of structure in mathematics. 


\section{Distributive Property}

The distributive property, which states that $a \times(b+c)=a \times b+a \times c$, is a particularly useful numerical property. The underlying principle of the vertical algorithm for whole-number multiplication which is commonly taught in U.S. elementary-school grades can be justified by the distributive property, and the property can be used to help young learners develop an expanded understanding of the meaning of multiplication beyond the concept of multiplication as repeated addition (Hurst \& Huntley, 2017; Kinzer \& Stafford, 2013). Kinzer and Stafford (2013) wrote that "the distributive property helps students understand what multiplication means, how to break down complicated problems into simpler ones, and how to relate multiplication to area by using array models" (p. 308).

Thinking about multiplication as "part-part whole reasoning with groups also enables children to use the distributive property of multiplication over addition" (Jacob \& Willis, 2003 p. 7) to compute products, such as $11 \times 6$. Rather than stacking these two numbers vertically with 11 at the top and arguing that the product of 11 and 6 is 66 because $6 \times 1=6$ and $6 \times 1=6$; it is more precise and accurate to use the distributive property to contend that the product of 11 and 6 is 66 because $11 \times 6=(10+1) \times 6=10 \times 6+1 \times 6=60+6=66$. (Powell, Ding, Wang, Craven, \& Chen, 2019).

Arithmetic patterns of calculations such as $7 \times 97+7 \times 3$ could be evaluated quickly using the distributive property rather than relying on order of operations. Kanbir et al. (2018) studied seventh-graders' knowledge and understanding of the distributive property. At the preintervention stage, none of the participants in Kanbir et al's study recognized that $7 \times 97+7 \times 3$ was equal to $7 \times(97+3)$, which equals $7 \times 100=700$. Kaminski (2002) studied the extent to which pre-service teachers used the distributive property when evaluating such expressions. 
Kaminski observed that although many pre-service teachers had heard of the distributive property, many were still not aware of how the property could be applied. "Kaminski's sample consisted of pre-service teachers, as opposed to in-service teachers but his observation begs the question as to whether or not the majority of in-service teachers would be clear about how the distributive property can be applied" (Hurst \& Huntley, 2017, p. 326).

\section{Pre-Service Teachers' Knowledge of Numerical Properties}

Fennema and Franke (1992) wrote that the teacher knowledge is one of the most important influences on what happens in the classroom and ultimately affects what students learn. Kanbir et al. (2018) and Ellerton and Clements (2011) asserted that many beginning teachers of mathematics in the elementary and middle-school grades do not have strong knowledge and understanding of the algebra content that they are expected to teach. Ellerton and Clements (2011) contended that elementary and middle-school teachers' poor knowledge of elementary numerical properties contributes to the issues facing beginning students of algebra. For teachers to be able to provide conceptually-rich algebra instruction in the elementary and middle-school grades they must possess strong knowledge and understanding of structure in early algebra (Barnett \& Ding, 2019; Ellerton \& Clements, 2011).

The scarcity of research investigating elementary and middle-school teachers' knowledge of properties of real numbers motivated me to complete two preliminary studies concerning aspects of that matter (Monandi, 2018, 2019). The preliminary studies revealed that participating preservice teachers had not yet developed strong knowledge and understanding of properties of real numbers, and could not consistently apply these properties in meaningful ways to solve and simplify algebraic problems. Other authors who have reported on matters related to preservice 
teachers' structure sense include Meixia Ding and her associates (Barnett \& Ding, 2019; Ding, 2016; Ding et al., 2013) and Kyungsoon Jeon (2012).

Ding and her associates (Barnett \& Ding, 2019; Ding, 2016; Ding et al., 2013) are among the few researchers who have investigated pre-service teachers' knowledge of number properties. Ding (2016) contended that the associative properties, together with the commutative properties and the distributive property, form the basic laws of arithmetic and are foundational to the learning of numerical concepts in the early grades. She added that these properties of numbers constituted fundamental mathematical ideas. She contended that in order to help young learners obtain meaningful initial learning of fundamental mathematical ideas it is essential for teachers (including preservice teachers) to develop strong knowledge of properties of real numbers.

Ding et al. (2013) examined preservice elementary teachers' (PETs') knowledge for teaching the associative property for multiplication. They observed that most PETs in their study did not have a conceptually-rich understanding of the associative property for multiplication. They wrote that most of the PETs could not come up with appropriate pictorial representations of the associative property for multiplication, and most were not able to use the associative property for multiplication in word problem contexts. Ding et al. also found that many PETs in their study misidentified the associative property as the commutative property. That same issue of misidentification was also observed in both of my preliminary studies (Monandi, 2018, 2019).

Jeon (2012) examined the knowledge that PETs exhibited regarding order of operations. Jeon found that PETs could recite the "Please Excuse My Dear Aunt Sally" (PEMDAS) mnemonic for order of operations but many did not know some of the exceptions associated with mnemonic. On the approach to finding the value of $7-3+11$, many of PETs in Jeon's (2012) report believed that the addition of 3 and 11 must be done first because addition, represented by 
"A" in PEMDAS, comes before subtraction, represented by "S" in PEMDAS. The PETs were not aware that addition and subtraction are of the same order and should be carried out as listed from left to right. Kanbir et al. (2018) argued that an overreliance on the PEMDAS mnemonic prevents young learners from developing strong understanding of structure in early algebra. This overreliance on PEMDAS was observed among seventh-grade students in their study. When the seventh-grade students were asked to find value of $4 \times\left(\frac{1}{4} \times 128\right)$ or the value of $7 \times 97+7 \times$ 3, they tended to rely on the PEMDAS mnemonic, and their responses indicated that they were not aware of the structure inherent in evaluating numerical expressions.

If PETs complete their teacher-education programs without a strong knowledge of structural aspects of early algebra, then their prospective students are likely to be greatly disadvantaged. Understanding structure in numerical relationships and knowing the relevance and usefulness of structural properties can support students' analysis of modeling situations. It could also support knowledge of variable manipulation (Kanbir et al., 2018; You, 2006). Ma (1999) also asserted that effective mathematics teachers of young learners needed to have strong knowledge of structure in mathematics.

The main conclusion from the studies of Ding and colleagues (Barnett \& Ding, 2019; Ding, 2016; Ding et al., 2013), together with Jeon's (2012) report and my two preliminary studies (Monandi, 2018, 2019), is that that preservice teachers have an inadequate understanding of properties of real numbers. Although Ding (2016) and Ding et al. (2013) focused only on the teaching of the associative property for multiplication, Barnett and Ding (2019) investigated the teaching of the associative property for addition and for multiplication in a natural classroom setting, and Jeon (2012) focused on preservice teachers' understanding of the order of operations. My two preliminary studies (Monandi, 2018, 2019) were centered on preservice 
teachers' knowledge of the following three properties: the associative property for addition, the associative property for multiplication, and the distributive property for multiplication over addition. In this dissertation study, I took a more comprehensive course and investigated preservice teachers' knowledge of the associative property for addition and multiplication, the commutative property for addition and multiplication, the distributive property, as well as their knowledge of additive and multiplicative inverse and identity properties.

\section{Theoretical Perspectives}

This dissertation study was influenced by mathematics education researchers (e.g., Kanbir et al., 2018; Presmeg, Radford, Roth, \& Kadunz, 2016) who have linked semiotic theories to mathematics teaching and learning and those who have written on the cognitive structures involved in learning (e.g., Gagne, 1985; Gagne \& Merrill, 1990; Gagne \& White, 1978; Tall \& Vinner 1981; Vinner \& Hershkowitz, 1980; Vinner \& Dreyfus, 1989). Semiotic

theories - those that emphasize the role of signs, and the way those signs communicate features about represented objects - were central to the framing of this study. Charles Sanders Peirce (1992, 1998) and Ferdinand de Saussure (1959) were the two most prominent figures taken into account. They are regarded as founders of this field of semiotics (Chandler, 2007; Presmeg et al., 2016).

The term semiotics is typically defined as the study of signs (Chandler, 2007; Presmeg, 2014; Presmeg et al., 2016). Beyond the understanding of semiotics as the study of signs, there is great variation in what leading scholars accept as constituting semiotics (Chandler, 2007). For example, Umberto Eco (1976) presented a very broad definition stating that "semiotics is concerned with everything that can be taken as a sign" (p. 7). Chandler (2007) described semiotics as the study that involves "not only of what we refer to as 'signs' in everyday speech, 
but of anything which stands for something else. In a semiotic sense, signs take the form of words, images, sounds, gestures and objects" (p. 2). I understand semiotics as encompassing signs that are part of some semiotic system in which those signs imbue certain meanings about objects within some accepted reality. Thus, semiotics allows us to ascertain how meanings are conceived and how reality is represented (Chandler, 2007). In the following sections, I describe the underpinnings of semiotic theories put forward by Peirce and de Saussure and describe how researchers have associated these theories with mathematics education and how these theories influenced my research for this dissertation.

\section{Charles Sanders Peirce and Ferdinand de Saussure}

Charles Sanders Peirce $(1992,1998)$ and Ferdinand de Saussure (1959) are both regarded as the co-founders in the field of semiotics (Chandler, 2007; Presmeg, 2014). Their theories emphasize the role of signs, and the way those signs communicate features about represented objects. According to Peirce $(1992,1998)$, a semiotic relation consisted of three basic tenets which form a sign relation: a sign, its object and its interpretant. A sign is anything that represents, and according to Pierce anything can be a sign. The object refers to the sign's subject matter. This could be anything that can be conceived from the sign. The interpretant refers to an individual's interpretation of the meaning of a sign — which may or may not be the object. Peirce explained that a sign relation is an irreducible triadic relation. That is, all three basic tenets of a semiotic relation must be considered to obtain the full meaning of the sign relation.

Instead of a triadic relation as theorized by Peirce $(1992,1998)$, de Saussure (1959) posited that the sign relation is dyadic, consisting of two basic tenets: the signifier and the signified. The signifier is the equivalent of a sign in Peirce's model, and the signified is the concept associated with the signifier - the meaning of the sign [i.e., the "object" with Peirce]. 
For example, de Saussure contended that a person cannot use the word "tree" without mentally envisioning the "concept of tree." In this example, the word "tree" is a signifier and a real-life tree or the mental image of "a tree" is the signified. For Peirce $(1992,1998)$, the same sign (the signifier) may signify different meanings for the same object (the signified) to different people (the referents). Hence, the need for a triadic relation. Peirce's model accounts for, and distinguishes, the sign itself, its subject matter (the object), and its assigned meaning (for an interpretant). Consider de Saussure's "tree" example above, the word "tree" can be regarded as a sign that directs someone to envision the abstract concept of "a tree" (the object), but in the realm of mathematics, specifically graph theory, the same sign can direct a person toward some other meaning (the interpretant) that fits within this realm (Kanbir, 2016; Kanbir et al., 2018).

\section{Semiotic Aspects of Early Algebra}

In this dissertation study, my perspective is that a fundamentally important issue to be associated with the learning of algebra is mainly semiotic. Semiotic aspects of early algebra can be categorized into two areas: those that arise from the structural aspect of early algebra, and those that stem from the symbolism and notation used in school algebra. To illustrate the semiotic features of structure in early algebra, I discuss an example presented by Kanbir et al. (2018). This example was discussed earlier to illustrate the meaning of structure sense. In their study, Kanbir et al. (2018) asked their seventh-grade student participants to "find the value of $4 \times\left(\frac{1}{4} \times 128\right)$ " without using a calculator. The statement "find the value of $4 \times$ $\left(\frac{1}{4} \times 128\right)$ " can viewed as a sign. This sign may signify different mathematical objects to different students. To some students, this sign may signify the application of the associative property for multiplication. To others, the same sign may signify the need to apply the mnemonic 
PEMDAS (Parenthesis, Exponent, Multiplication, Division, Addition, and Subtraction). Many things influence the meaning learners ascribe to signs (Kanbir et al., 2018).

The second aspect of semiotics in early algebra is evident in the symbolism and notation used in algebra. As students transition from arithmetic to algebra, they encounter new symbols and notations. From this theoretical perspective, I contend that students' difficulties in school algebra can be partly attributed to the notation and symbolism in algebra. This should not be taken to mean that the defining feature of algebra is its notational system and symbolism. I am asserting that mathematics is a language with its own syntax and structure, and when young learners are met with new notation and symbolism it can be challenging for them to cope with it (Blanton et al., 2015; Stacey \& MacGregor, 2000; Swafford \& Langrall, 2000).

\section{Semiotic Aspect of Properties of Real Numbers}

From the Peircean perspective, the statement "find the value of $4 \times\left(\frac{1}{4} \times 128\right)$ " can viewed as a sign, which was framed with a certain mathematical object in mind-the associative property for multiplication. Those who attempt to find the value by first computing $\frac{1}{4} \times 128$ see the sign but fail to recognize the mathematical object behind the sign. Those who respond by first computing $\frac{1}{4} \times 128$ probably do so because of the mnemonic PEMDAS ("Please Excuse My Dear Aunt Sally"), which is commonly taught in U.S. elementary and middle-school grades as a way to recall the order of operations four use in complex calculations (Kanbir, 2016; Kanbir et al., 2018). The mnemonic PEMDAS is meant to represent the following: Parentheses ("the operations within any parentheses should be dealt with first"), Exponents ("exponents are dealt with second"), Multiplication or Division ("thirdly, multiplication or division should be dealt 
with as listed from left to right"), and Addition or Subtraction ("lastly, addition or subtraction should be dealt with as listed from left to right").

An overreliance on PEMDAS can prevent young learners from developing number structure sense which is necessary for their later success in algebra and in higher mathematics (Barnett \& Ding, 2019; Kanbir, 2016; Kanbir et al., 2018). "Without number structure sense and with rigid memorization of the order of operations demanded by PEMDAS, students can not only get wrong answers for calculations, but also fail to learn important structural principles" (Kanbir, 2016, p. 43). Consider the following task presented to pre-service teacher participants in this study: "Without using a calculator find the value of $0.6 \times 250+250 \times 0.4$ and explain how you got your answer." Most of the pre-service teacher participants did not recognize the sign inherent in this task; they did not recognize the sign has having anything do with a structural property of real numbers. To most pre-service teacher participants, the sign " $0.6 \times 250+250 \times$ 0.4 " directed them to the mnemonic PEMDAS for order of operations. Because of the popularity of the mnemonic, the most common strategy for evaluating $0.6 \times 250+250 \times 0.4$ was computing the multiplications first and then carrying out the additions. Those who recognized that the mathematical object under consideration for the sign " $0.6 \times 250+250 \times 0.4$ " was the distributive property would have known that $0.6 \times 250+250 \times 0.4$ is equal to $250 \times(0.6+0.4)$ and would have used this fact to evaluate the expression.

It is possible to evaluate $4 \times\left(\frac{1}{4} \times 128\right)$ or $0.6 \times 250+250 \times 0.4$ using the concept of order of operations and obtain a correct answer if calculation are done accurately. Those who use this method may obtain correct answers but would not have "attended to structure inherent in the calculation" (Kanbir, 2016, p. 43). The expressions $4 \times\left(\frac{1}{4} \times 128\right)$ and $0.6 \times 250+250 \times 0.4$ were written with certain mathematical objects in mind and obtaining correct answers is not the 
object of the signs. Thus, students relying on order of operations to evaluate these expressions have not attended to the structural properties inherent in the calculation. Such students did not “give appropriate meanings to signs, where the word 'appropriate' is to be interpreted as meaning 'consistent with the goals of the curriculum."' (Kanbir, 2016, p. 61). Kanbir (2016) asserted that a major task for mathematics teachers is to "assist learners to recognize signs, give the signs appropriate meanings, and then do the mathematics demanded by what is signified in the tasks in which the signs are present" (p. 61).

\section{Cognitive Structures Involved in Learning}

As discussed earlier, the sign "find the value of $4 \times\left(\frac{1}{4} \times 128\right)$ " may signify different mathematical objects to different students. Kanbir (2016) and Kanbir et al. (2018) discussed three examples of hypothetical students responding to this sign. Upon seeing $4 \times(1 / 4 \times 128)$, one hypothetical student may immediately be directed to think of PEMDAS and begin by first computing what is inside the parentheses. A second hypothetical student may see the same sign and think to associate 4 and $1 / 4$. A third hypothetical student might think: "OK-the teacher wants to see if 1 recognize that the associative property for multiplication should be used." (Kanbir et al., 2018, p. 102). From a mathematics perspective, the reasoning of the second and third hypothetical student is preferable compared to that of the first student (Kanbir, 2016; Kanbir et al., 2018). A distinction is made between the reasoning of the second and third hypothetical student because it is also possible for a student to associate 4 and $1 / 4$ in $4 \times$ $\left(\frac{1}{4} \times 128\right)$ but not connect that with the words "associative property for multiplication."

In the preceding paragraph, the three hypothetical students responded to the same sign in different ways. For Kanbir (2016) and Kanbir et al. (2018), this led them to ask the following 
question: "Why do students respond to the same sign in different ways?" Kanbir and colleagues contended that "with respect to any concept it is likely that different students will have different 'cognitive structures,' and that fact will result in their interpreting the signs in different ways" (Kanbir et al., 2018, p. 103). Kanbir and colleagues (Kanbir, 2016; Kanbir et al., 2018) drew from Herbart's theory of apperception to explain why the same sign might be interpreted differently by the different learners. According to Herbart (1898), the apperception of a learner with respect to some concept is influenced by the prior learning experiences of the learner. So, according to Kanbir and colleagues (Kanbir, 2016; Kanbir et al., 2018), the learner's experiences would influence the way that the learner responds to the sign, depending on how the learner's mind has been structured based on prior experiences. Thus, those prior learning experiences would mean that different learners will develop different cognitive structures leading to different ways of interpreting the same sign. The goal for teachers of mathematics is to help young learners "learn to recognize signs, process their meanings appropriately, and then respond to tasks, and even create tasks, in which the signs are used" (Kanbir et al., 2018, p. 103).

Of interest in this dissertation study is the knowledge that pre-service teacher participants have about structural properties of real numbers. Kanbir and colleagues (Kanbir, 2016; Kanbir et al., 2018) showed that middle-school students in their studies did not demonstrate as strong a knowledge of structural properties as would be expected of them according to Standards documents (NCTM, 2000; NGA \& CCSSO, 2010). Part of the problem could be attributed the mathematical experiences that young learners of mathematics receive in the elementary and middle-school grades (Ellerton \& Clements, 2011; Kanbir, 2016; Kanbir et al., 2018). Ellerton and Clements (2011) asserted that many beginning teachers of mathematics did not have wellformed knowledge of the concepts that they were expected to teach. Many beginning (and pre- 
service) teachers may not be able to respond appropriately to signs such as $4 \times\left(\frac{1}{4} \times 128\right)$ and $0.6 \times 250+0.4 \times 250$. The research of Ding and colleagues (Barnett \& Ding, 2019; Ding, 2016; Ding et al., 2013) generated results which supports that assertion.

To ascertain what pre-service teacher participants knew and understood about properties, I needed to consider their cognitive structures with respect to properties of real numbers. Prior learning experiences would have shaped their existing cognitive understandings of structural properties. To describe the extent to which pre-service teacher participants knew and understood properties of real numbers, I examined the findings of this study by relying on theories on cognitive structures which linked Peirce's concepts of "signifier" and "mathematical object" as posited by Kanbir and colleagues (Kanbir, 2016; Kanbir et al., 2018). Researchers who have explored the cognitive structures involved in learning include Robert Gagné and colleagues (Gagné, 1985; Gagné \& Merrill, 1990; Gagné \& White, 1978) and Shlomo Vinner and colleagues (Tall \& Vinner 1981; Vinner \& Hershkowitz, 1980; Vinner \& Dreyfus, 1989).

Gagné and White (1978) posited that a learner's cognitive structure is comprised of four separable components: verbal knowledge, intellectual skills, imagery and episodes. With respect to structural properties, verbal knowledge could be demonstrated by one's capacity to recall the definition or meaning of a structural property; intellectual skills could be demonstrated by one's capacity to recognize and apply a structural property in relevant situations because he or she understands that structural properties supersede PEMDAS rules for order of operations in certain situations; imagery could refer to one's capacity to recognize and respond to tasks, or create tasks, with patterns of arithmetic calculations for which applying a structural property is appropriate; and episodes refers memories to prior learning experiences in which a structural property was used or explained (Kanbir, 2016; Kanbir et al., 2018). In addition to these four 
components of a learner's working memory, Gagné and colleagues (Gagné, 1985; Gagné \& Merrill, 1990) added attitudes and motor skills to the list. In this dissertation, the cognitive structures of participating pre-service teachers were described in terms of verbal knowledge, intellectual skills, imagery, and episodes, with respect to their demonstrated knowledge of properties of real numbers.

Vinner and colleagues (Tall \& Vinner 1981; Vinner \& Hershkowitz, 1980; Vinner \& Dreyfus, 1989) advanced the ideas of concept definition and concept image in order to connect an individual learner's cognitive structure to his or her own unique understanding a mathematics concept or principle. Tall and Vinner (1981) described the term concept image as "the total cognitive structure that is associated with the concept, which includes all the mental pictures and associated properties and processes" (p. 152). They added: "The definition of a concept (if it has one) is quite a different matter. We shall regard the concept definition to be a form of words used to specify that concept. It may be learnt by an individual in a rote fashion or more meaningfully learnt and related to a greater or lesser degree to the concept as a whole" (Tall \& Vinner, 1981, p. 152 , original emphasis).

A student who upon seeing the sign " $4 \times(1 / 4 \times 128)$ " connects it to, and explicitly states, the associative property for multiplication would have demonstrated a well-formed knowledge of the concept image of the property. A student who can recite the description of the associative property for multiplication as $a \times(b \times c)=(a \times b) \times c$ but does not connect this property with the sign " $4 \times(1 / 4 \times 128)$ " would not have demonstrated a well-formed concept image of the property. A student who cannot state definition or meaning of the associative property for multiplication and cannot even recognize that 4 and $1 / 4$ in $4 \times(1 / 4 \times 128)$ can be associated would not have demonstrated a strong knowledge of the concept image associated with the property. 
In this study, some pre-service teacher participants recognized that the 4 and the $1 / 4$ in $4 \times$ $(1 / 4 \times 128)$ could be associated but did not explicitly connect this association with the expression “associative property for multiplication." It was also observed some participating pre-service teacher interviewees provided appropriate examples of the distributive property but did not recognize arithmetic patterns of calculations, such as $7 \times 97+7 \times 3$, for which using the distributive property would be appropriate. This affirmed Kanbir and colleagues' assertion that a learner's "concept image with respect to some topic is not made up solely of separate components (verbal, skills, imagery, episodes, [and] attitudes). Cognitive links, or relationships between the components, are also important in defining the concept image" (Kanbir et al., 2018, p. 164).

Also of interest in this dissertation study was whether participating preservice teachers responded, both receptively and expressively, to signs for which the underlying mathematical objects were the properties of real numbers under consideration. The words "receptively" and “expressively” were used in manner consistent with Ellerton and Clements's (1991) receptive and expressive language and Del Campo and Clements's (1987) receptive and expressive modes of communication. Receptive language involved the 'processing of someone else's “communication" and expressive language the use of one's "own language" (Kanbir et al., 2018, p. 107). With respect to structural properties, receptive communication pertained to participating preservice teachers' capacity to state accurately or write down the meaning of a structural property based on what was learned; expressive communication pertained to participating preservice teachers' capacity to describe accurately the meaning of a structural property, and their capacity to apply the property in appropriate but extemporaneous situations (Kanbir, 2016; Kanbir et al., 2018). 


\section{Summary}

In this chapter, I reviewed the literature which informed the framing of this dissertation study. This study is framed with the perspective that students should be enabled opportunities to interact with "mathematical structure, at every age and stage of their exposure to mathematics and to mathematical thinking" (Mason, Stephens, \& Watson, 2009, p. 11). This perspective regarding the importance of mathematical structure to mathematical understanding has also been put forward by other researchers including Hoch and Dreyfus (2004), Kanbir, Clements, and Ellerton (2018), Ma (1999), and Novotna \& Hoch (2008). This study is concerned with the structure sense of prospective elementary-school teachers; their knowledge and understanding of properties of real numbers, and their capacity to apply these properties when computing with real numbers or when solving or simplifying algebraic expressions. Prior studies (Barnett \& Ding, 2019; Ding, 2016; Ding et al., 2013; Monandi, 2018, 2019) suggest that prospective elementaryschool teachers do not have strong knowledge or understanding of properties of real numbers.

In this chapter, I also discussed the theoretical perspectives which influenced this study. This dissertation study was influenced by mathematics education researchers who have linked semiotic theories to mathematics teaching and learning (e.g., Kanbir et al., 2018; Presmeg et al., 2016). Semiotic theories - those which emphasize the role of signs, and the way those signs communicate features about represented objects-were central to the framing of this study. To describe the extent to which pre-service teacher participants knew and understood properties of real numbers, I examined the findings of this study by relying on theories on cognitive structures that linked Peirce's concepts of "signifier," "interpretant," and "object" as posited by Kanbir and colleagues (Kanbir, 2016; Kanbir et al., 2018). 


\section{CHAPTER III: RESEARCH METHODS}

This dissertation study is a mixed-methods investigation. Both quantitative and qualitative methods for data analysis are utilized. Quantitative data were collected from administered paper-and-pencil instruments which contained 15 questions evaluating pre-service teachers' knowledge of, and capacity to apply, properties of real of numbers in solving and simplifying algebraic problems. The participants in the study were prospective elementaryschool teachers. Qualitative data were collected from participants' written responses to questions on the paper-and-pencil instruments and from audio-taped interviews with a small number of prospective teachers who agreed to be interviewed. Audio-taped interviews provided added insight into the participants' knowledge and understanding of properties of real numbers.

\section{Setting up for the Study}

During the dissertation proposal stage, the goal was to conduct a study that described the knowledge and understanding that prospective elementary-school teachers have about properties of numbers, and study participants were to be sought from three different mathematics courses for pre-service elementary-school teachers (PETs). The initial plan, at the proposal stage, for this dissertation study was to collect data on PETs' knowledge by to issuing a mathematics assessment on number properties in three mathematics courses for PETs and to conduct interviews with some of the prospective teachers. The goal was to examine, assess and describe prospective elementary-school teachers' knowledge of, and capacity to apply, structural properties of real numbers when simplifying and evaluating mathematical expressions. However, because the data from the preliminary studies (Monandi, 2018, 2019) had shown that prospective teachers did not demonstrate strong knowledge or understanding of properties of numbers, it was decided that this dissertation study should include some type of intervention. 
The dissertation study was centered on three mathematics courses for prospective elementary-school teachers that PETs are expected to take in succession. The profiles of these three courses are presented below.

\section{Mathematics courses for PETs}

The first mathematics course for PETs (hereafter- "MT1") that was included in this study is meant to be first mathematics course that prospective elementary and early childhood education teachers are required to take at this community college. In the college's course catalog, this course is described as focusing on problem-solving techniques, decimal and non-decimal numeral systems, mental computations, estimation, set theory, numerical reasoning, and algebraic reasoning.

The second mathematics course (hereafter_ "MT2") for PETs that was included in this study is supposed to be taken by prospective teachers after completing MT1. Although the MT1MT2 sequential order is required, PETs can take the courses out of order by obtaining permission. In the college's course catalog, this course is described as focusing on investigating measurement, two- and three-dimensional shapes, and geometrical concepts.

The third mathematics course (hereafter- "MT3") for PETs that was included in this study is supposed to be taken after prospective teachers have completed both MT1 and MT2. MT1 and MT2 are listed as prerequisites for enrollment in MT3 in the college's course catalog. However, PETs are allowed to be concurrently enrolled MT2 and MT3 in the same semester. None of the participating PETs were concurrently enrolled. In the course catalog, MT3 is described as focusing on statistics topics including data collection, data interpretation, sampling, correlation, regression, distributions, and probability. 


\section{Teaching Aspect of Study}

The teaching intervention was situated in the MT1 course because part of the course's learning outcomes included fostering numerical and algebraic reasoning. There was no teaching intervention conducted in the MT2 and MT3 courses. Participating PETs in these two courses completed Assessment I (see Appendix C) as part of the coursework and one prospective teacher from each of these two courses consented to and participated in an audio-taped interview. MT2 and MT3 PETs completed their assessment approximately midway through the fall 2019 semester. The audio-taped interviews with MT2 and MT3 PETs were also completed approximately midway through the fall 2019 semester at a time convenient for both the interviewer and the interviewee. I, the researcher, was the interviewer.

Participating PETs in the MT1 course participated in lessons that emphasized properties of real numbers and completed pre-teaching and post-teaching assessments. Two MT1 participants also agreed to participate in pre-teaching and post-teaching audio-taped interviews. With the MT1 course, instruction on properties of real numbers was introduced as part of the unit on operating on whole numbers. Participating PETs in the MT1 course completed Assessment I—used as the pre-teaching assessment—in late September 2019 prior to the start of the unit on whole numbers and whole-number operations. After introducing whole numbers in MT1 and modeling addition and subtraction with whole numbers, PETs in MT1 were tasked with completing a chart (see Appendix D) in which they described which properties of operations were true for whole-number addition and subtraction.

Whole-number addition and subtraction was followed by instruction and discussion on the meaning of multiplication and division, and ways of modeling these operations with whole numbers. PETs in MT1 were then tasked with completing another chart (see Appendix D) in 
which they described properties of operations which were true for whole number multiplication and division. Following completion of the whole-number unit, the discussion on number properties continued with operations on fractions, decimals, and integers. Participating PETs in the MT1 course completed Assessment II — used as the post-teaching assessment — in late November 2019 following the completion of the course module on real numbers and operating on real numbers.

Challenges to implementing an instructional intervention. The dissertation study was centered on three mathematics courses for PETs offered at a two-year community college. The intervention was situated in the first of these three mathematics courses for PETs. This college, like most two-year colleges, have articulation agreements with four-year institutions so their students can smoothly transfer their courses when they enroll in the four-year institutions. Articulation agreements are formed typically "between a community college and a four-year institution with the goal of creating a seamless transfer process for students" (Barrington, 2020, para. 7). "Not every community college has them, but most do, and they are an invaluable resource for transfer students" (Barrington, 2020, para. 2). To form and maintain articulation agreements, courses at this community college - whose students are subject in this investigation — are required to go through a formal process of approval and have a syllabus and course schedule recorded with the college curriculum committee [pseudonym]. Deviations from the syllabus and course schedule require approval of the college curriculum committee. This constrained me from making changes to course schedule. Instead, the teaching intervention in the MT1 course involved giving properties of real numbers an elevated focus during instruction on operating on real numbers (whole numbers, integers, fractions and rational numbers, and decimals) - topics that were already part of the course. 
Instructional intervention in the MT1 course. Lessons emphasizing properties of

operations were introduced during instruction on whole number operations. Kanbir (2016) and Kanbir et al. (2018) underscored the need for elementary and middle-school teachers trained to capitalize on the algebraic nature of arithmetic and early mathematics. Accordingly, this includes giving special attention to number properties as part of early algebra instruction (Kanbir, 2016;

Kanbir et al., 2018).

\begin{tabular}{|c|c|c|c|}
\hline Week & Session \# & Topics Covered & Assignments, Assessments, Activities \\
\hline \multirow[t]{2}{*}{4} & 7 & \multicolumn{2}{|c|}{ Pre-Teaching Assessment for Dissertation Study was issued } \\
\hline & 8 & Set Theory, Venn Diagrams & Homework 3 due \\
\hline \multirow[t]{2}{*}{5} & 9 & Catch Up/ Review for Exam 1 & \\
\hline & 10 & \multicolumn{2}{|c|}{ Exam 1: Problem Solving through Venn Diagrams } \\
\hline \multirow[t]{2}{*}{6} & 11 & Addition of Whole Numbers & Addition Properties \\
\hline & 12 & Subtraction of Whole Numbers & \\
\hline \multirow[t]{2}{*}{7} & 13 & Multiplication of Whole Numbers & $\begin{array}{l}\text { Multiplication Properties, } \\
\text { Distributive Property }\end{array}$ \\
\hline & 14 & Division of Whole Numbers & \\
\hline \multirow[t]{2}{*}{8} & 15 & Divisibility and Factorization & Homework 4 due \\
\hline & 16 & GCF and LCM & Quiz 4 \\
\hline \multirow[t]{2}{*}{9} & 17 & \multicolumn{2}{|l|}{ Catch Up/ Review for Exam 2} \\
\hline & 18 & \multicolumn{2}{|c|}{ Exam 2: Whole Number Arithmetic through GCF and LCM } \\
\hline \multirow[t]{2}{*}{10} & 19 & Integer Models and Arithmetic & \\
\hline & 20 & Integer Models and Arithmetic & The Role of Properties, Homework 5 due \\
\hline \multirow[t]{2}{*}{11} & 21 & Fraction Models \& Arithmetic & \\
\hline & 22 & Fraction Addition/Subtraction & The Role of Properties, Homework 6 due \\
\hline \multirow[t]{2}{*}{12} & 23 & Fraction Multiplication/Division & \\
\hline & 24 & Decimal Numbers \& Arithmetic & The Role of Properties, Homework 7 due \\
\hline \multirow[t]{2}{*}{13} & 25 & Decimal Arithmetic & Quiz 5, Exam 3 Review issued \\
\hline & 26 & \multicolumn{2}{|c|}{ Post-Teaching Assessment for Dissertation Study was issued } \\
\hline
\end{tabular}

Figure 1. MT1 course schedule.

For the instructional intervention, it was desired to incorporate the model lessons and materials on numerical structure in the Kanbir (2016) and Kanbir et al. (2018) studies, but the rules regarding using college-approved syllabus and course schedule limited the desired comprehensive overhaul of teaching in MT1. Instead, as stated earlier, the intervention in MT1 
involved giving numerical structure sense an elevated focus during regular instruction called for in the college-endorsed course schedule.

The MT1 course schedule is given in Appendix E. A portion of this course schedule is presented in Figure 1. The MT1 course met twice a week for 15 weeks of classroom instruction, and each meeting was 105 minutes long. Lessons on whole numbers were introduced during the sixth week of the semester. Whole number lessons started with instruction and discussion on whole number addition. A handout (see Appendix F) was issued to PETs for completion as part of the lesson on whole number addition. The goal was for PETs to learn ways of modeling whole number addition, explore strategies and algorithms for adding whole numbers, and to explain and justify why those strategies and algorithms work by making connections to the role of place value and properties of operations. PETs completed the following tasks, in groups of three or four, as part of classroom instruction on whole number addition. During all classroom instruction, PETs were regularly provided opportunities to discuss ideas and concepts in small groups before reporting their groups' findings to classmates. After completing these tasks, PETs were tasked with completing the table in Appendix D on properties of whole number addition.

\section{Classroom Tasks (Whole Number Addition)}

DIRECTIONS: Get into groups of three or four and work on the following tasks. Discuss the questions, work out what is asked, determine the meaning of each, and answer appropriately.

1. Use a number line to find the sum of $5+3$ and $3+5$. What do you find? What can you conjecture about the addition of whole numbers?

- Let $a$ and $b$ be two unique whole numbers. Use a number line to find the sum of $a+b$ and $b+a$ ? What do you find? Repeat this previous step a few more times with different numbers for $a$ and $b$. What can you conclude about the addition of whole numbers?

2. What would be a quick way of determining the value of $99+234+1$ without using a calculator or writing anything down? What did you do and is it related to what you concluded about the addition of whole numbers from above? 
3. If $a=12, b=6$ and $c=2$, what are the values of each of the following?

$(a+b)+c$

$a+(\mathrm{b}+c)$

- Repeat this, only this time let $a=51, b=26$, and $c=12$. What do you find? What can you conjecture about the addition of whole numbers?

- Repeat again, only this time let a, b, and c be any whole numbers that your group chooses. What do you find? What can you conclude about the addition of whole numbers?

4. Do you think $a+b$ will always equal $b+\mathrm{a}$, no matter which numerical values you allow $a$ and $b$ to be? Do you think $a+(b+c)$ will always equal $(a+b)+c$, no matter which numerical values you allow $a, b$ and $c$ to be?

5. In adding 957 and 384, will the strategy of adding 1200, 130, and 11 give the right answer? If so, why? Discuss the role of place value and whether any addition property is used.

6. You may have heard in your prior mathematics education that "when you add number and zero, the result is the number itself." Have you heard this or some similar statement? What does it mean to you? Is it true always? How can we state this concisely?

7. Is the sum of two whole numbers also a whole number? Is this always true for any two whole numbers? Is this always true when adding three or more whole numbers?

Whole number lessons continued with instruction and discussion on whole number subtraction. Another handout (see Appendix F) was issued for PETs to complete as part of the lesson on whole number subtraction. PETs were expected to learn ways of modeling whole number subtraction and explore strategies and algorithms for subtracting whole numbers. PETs were challenged to explain and justify strategies and algorithms for whole number subtraction. Like with whole number addition, PETs were expected to make connections to the role of place value and, possibly, properties of operations when explaining and justifying strategies and algorithms for subtracting whole numbers. PETs completed the following tasks as part of classroom instruction on whole number subtraction. 


\section{Classroom Tasks (Whole Number Subtraction)}

DIRECTIONS: Get into groups of three or four and work on the following tasks. Discuss the questions, work out what is asked, determine the meaning of each, and answer appropriately.

1. Let $a$ and $b$ be two unique whole numbers. Use a number line to find the value of $a-b$ and $b-a$ ? What do you find? Repeat this with different numbers for $a$ and $b$. What can you say about the subtraction of whole numbers? Contrast this with what you found regarding addition of whole numbers?

2. If $a=12, b=6$ and $c=2$, what are the values of each of the following?

$(a-b)-c$

$a-(\mathrm{b}-c)$

- Repeat this, only this time let $a=51, b=26$, and $c=12$. What do you find? What can you conjecture about the addition of whole numbers?

- Repeat again, only this time let a, b, and c be any whole numbers that your group chooses. What do you find? What can you say about the subtraction of whole numbers? Contrast this with what you found regarding addition of whole numbers?

3. In subtracting 214 and 148 , will the strategy of adding $100,-30$, and -4 give the right answer? If so, why?

4. Explain why subtracting 2462 from 4835 is the same as

a. Subtracting 2432 from 4835, and then subtracting 30 more.

b. Subtracting 2462 from 4862, and then subtracting 27 more.

c. Subtracting 2400 from 4800 and then subtracting 27 more.

5. Previously, we saw that $a+0=0+a=a$ for any $a$ that is a whole number. Now, let $a$ be any whole number that your group chooses? What is the value of $a-0$ ? What is the value of $0-a$ ? What can you say about subtraction and contrast it to what you concluded about addition?

6. Let $a$ and $b$ be two unique whole numbers. Is the value of $a-b$ always a whole number? Explain. Contrast it what you found regarding addition.

After completing these tasks, PETs were tasked with completing the table in Appendix D on whether the properties for whole number addition also hold for whole number subtraction.

During the seventh week of the semester, multiplication and division of whole numbers were the topics of the week. Handouts for whole number multiplication and for division (see 
Appendix F) were issued to PETs to complete. PETs learned about the different ways of modeling whole number multiplication and division and investigated common algorithms used when multiplying and dividing whole numbers. PETs were tasked with explaining the logic and reasons why those common algorithms worked. PETs were also tasked with computing whole number multiplication and division without relying on algorithms. They were expected to use invented strategies and were also expect to provide explanations and justifications for why those strategies were valid. The goal was to provide PETs opportunities to interact with, and use, properties of whole numbers multiplication and introduce the distributive property and highlight its applications. PETs completed the following tasks on whole number multiplication.

\section{Classroom Tasks (Whole Number Multiplication \& Properties)}

DIRECTIONS: Get into groups of three or four and work on the following tasks. Discuss the questions, work out what is asked, determine the meaning of each, and answer appropriately.

1. Without using a calculator or relying on any algorithm, compute each of the following products in at least three different ways. State any properties that you use.
a. $24 \times 25$
b. $120 \times 325$

2. How could you use the stated property to evaluate $12 \times 250$ ?

a. How could you use an associative property to find the value of $12 \times 250$ ?

b. How could you use the distributive property to find the value of $12 \times 250$ ?

3. How could you use the distributive property to find the value of $17 \times 97+17 \times 3$ ?

4. Which property would you use to find the value of $p$ ? $16 \times 28=11 \times 28+p \times 28$.

5. How would you quickly determine the value of $25 \times 37 \times 4$ ? Which property (or properties) would you be applying?

After working and interacting with whole number division, PETs were tasked with completing the table in Appendix D on whether the properties for whole number multiplication 
also hold for whole number division. PETs completed the following tasks on whole number division. These tasked focused on emphasizing the meanings of whole number division.

\section{Classroom Tasks (Whole Number Division)}

DIRECTIONS: Get into groups of three or four and work on the following tasks. Discuss the questions, work out what is asked, determine the meaning of each, and answer appropriately.

Question 1: Applewood Elementary School has just bought 24 computers for its 4 fifthgrade classes. How many computers will each classroom get? Use a diagram or a picture to illustrate your answer. Describe in words how you arrived at your answer.

Question 2: Carlos has 24 apples with which to make apple pies. If it takes 4 apples per pie, how many pies can he bake? Use a diagram or a picture to illustrate your answer. How might your reasoning for this problem differ from Question 1? Explain your method.

During the tenth week of the semester, the number system was expanded to include "negative numbers" and instruction included working with and operating on the set of integers: $\mathbb{Z}$ $=\{\ldots-3,-2,-1,0,1,2,3 \ldots\}$. During instruction on integers and integer operations, PETs were challenged to examine the properties of whole number operations and determine whether those properties can be extended to the set of integers. The additive inverse property was introduced during this week after introducing the concept of negative numbers and defining the opposite of a number. The integers handout issued to PETs is given in Appendix F.

During the eleventh week of the semester, the number system was further expanded to include "fractional numbers" and introduced the set of rational numbers: $\mathrm{Q}=\left\{\frac{p}{q} \mid p, q \in \mathbb{Z}, q \neq\right.$ $0\}$. As PETs interacted with and operated on fractions and rational numbers, they were tasked with examining the properties of operations and whether those properties could be extended to the set of rational numbers. The multiplicative inverse property was introduced during this week after the concept of reciprocal was discussed. The fractions handout issued to PETs is also provided in Appendix F. 
During the twelfth week of the semester, the number system was extended to include any number that can be plotted on a real number line to form the set of real numbers $\mathbb{R}$. Decimals were introduced and relationship between fractions and decimals was explored. After working with and operating on decimals and real numbers, PETs were once again challenged to examine the properties of rational numbers and determine whether those properties can be extended to the set of real numbers. The handout on decimals and real numbers issued to PETs is provided in Appendix F as well.

\section{Participants, Instrumentation, and Procedures}

\section{Participants}

The participants in the study consisted of a convenience sample of prospective elementary-school teachers (PETs) enrolled in mathematics courses offered at a community college in the Mid-Atlantic region of the United States of America. No measures were used to control who enrolled in the courses. Participants were sought from three courses for PETs: MT1, MT2, and MT3. The decision to include participants from three different mathematics courses for teachers was made because I wanted to compare MT1 PETs' knowledge of number properties to MT2/MT3 PETs' knowledge. The number of participants in the study was dependent upon student enrollment and willingness to participate. Institutional Review Board (IRB) approval for this study was obtained. The procedures and process that were required by the

college and the department to engage students in the project were followed, with consent from all participants being obtained before the start of the study. 


\section{Instrumentation}

Two paper-and-pencil assessments (see Appendices B and C) were designed for use in this study. There were 15 items on each of the assessments. The paper-and-pencil assessments utilized replicated and modified items that were used in the Kanbir et al. (2018) study and in my two preliminary studies (Monandi, 2018, 2019). Permission to replicate, modify, and utilize items from these studies was sought and granted. Participants were encouraged not to erase if they believe they had made an error in responding to a question on the paper-and-pencil assessments. Instead, they were instructed to draw one line through the erred sentence or inscription and proceed to write what they believed to be correct or appropriate. Participants were allotted 50 minutes to complete the paper-and-pencil instruments.

Paper-and-pencil assessment instruments. The main test instruments in this study were the paper-and-pencil assessment instruments. Two parallel versions (Assessment I and Assessment II — see Appendices B and C) were reproduced for use this study. Assessment I was used as the pre-teaching instrument for MT1 participants and was also used as the sole instrument for MT2 and MT3 participants. Assessment II was used as the post-teaching instrument for MT1 participants. There were 15 questions on the paper-and-pencil assessments. Question 1 and Question 12 were included to assess whether participants could appropriately describe a mathematical property in words.

Question 1. A really important property for numbers and for algebra is called the commutative property for multiplication. Describe this property.

Question 12. Another important property for numbers and for algebra is called the associative property for addition. Describe this property. 
These two questions were identical on both instruments. They were scored based on correctness. If a response to each of these questions was considered appropriate, it was marked as correct.

There were nine questions on the paper-and-pencil instruments which were included to assess whether participating PETs could recognize and use number properties appropriately in order to simplify expressions or solve equations and to assess whether they could identify the properties by name. These questions are hereafter referred to as the "nine property questions." For some of these nine property questions (NPQs), participating PETs were expected to present two different ways of solving the problems. Each of the nine property questions could be addressed by using a number property. Question 2 is an example of one of the nine property questions for which participants were expected to solve the problem in two different ways. It is possible that a student may solve this question by relying on order of operations, but that does not mean that the same student is unaware of the usefulness of the associative property for addition in addressing this problem. Because of this, the student is given an opportunity to describe an alternative way to see if he or she might address the question by applying the associative property for addition. Question 2, Question 3, and Question 4 are examples of the nine property questions on Assessment I (pre-teaching version). Parallel questions were written for Assessment II (post-teaching version).

Question 2. Suppose you were asked to calculate the value of $940+(60+427)$ in your head. How would you do it, and which property would you be using? Can you think of a different way of evaluating $940+(60+427)$ ? Explain.

Question 3. If $13 \times y=9 \times 13$, what must $y$ equal?

Which property allows you quickly to determine the value of $y$ ? 
Question 4. What must $x$ equal if $3 x=21 \times 3+4 \times 3$ ?

Explain how you got your answer.

Is there another way of finding the value of $x$ if $3 x=21 \times 3+4 \times 3$ ? Explain.

These nine property questions received three different scores: correctness, usage of property, and correct identification of property used by name.

Question 6 and Question 13 were included to assess whether participating PETs would inappropriately apply a number property to an operation for which the property does not hold.

Question 6. What must $x$ equal if $12-(8-4)=(12-x)-4$ ?

Explain how you got your answer.

Question 13. What must be the value of $y$ if $(10-y)-(10-5)=0$ ?

Explain your answer.

Question 14. Terry was solving the problem below. Each line shows the property used to move from one step to the next. Write the appropriate expression for each step.

$$
45+(56+-45)
$$

a.

b.

c.

d.

e.

Question 15. Jackie was solving the problem below and completed it as follows. Provide the appropriate property that Jackie applied to move from one step to the next step.

$$
3 \times 10 \times \frac{1}{3} \times 1.5
$$

Commutative property for addition Associative property for addition Additive Inverse Property Additive Identity Property 

a. $3 \times\left(10 \times \frac{1}{3}\right) \times 1.5$
b. $3 \times\left(\frac{1}{3} \times 10\right) \times 1.5$
c. $\left(3 \times \frac{1}{3}\right) \times 10 \times 1.5$
d. $\quad 1 \times 10 \times 1.5$
e. $10 \times 1.5$
f. $\quad 15$

Question 6 and Question 13 were scored based on correctness. If a response to each of these questions was considered appropriate, it was marked as correct. Question 14 and Question 15 assessed whether participating PETs could explain using number properties, the steps taken by some hypothetical students in evaluating numerical expressions. Each of Question 14 and Question 15 was worth one point. For Question 14, one-quarter points were given for each step identified correctly. For Question 15, one-fifth points were given for each step. Parallel versions of these questions were written for Assessment II. The assessments in this study were intended to evaluate what participating PETs knew and understood about properties of real numbers.

Each assessment completed by the participating PETs was evaluated and assigned four different scores: an overall score for the entire assessment, a score on the nine property questions, a score on the usage of properties, and a score on identifying properties by name. The overall score was determined by scoring all 15 questions based on the correctness of the responses to each question on the paper-and-pencil assessments. The maximum possible points for the overall score was 15 . For the nine property questions, each question was scored for correctness, application of the appropriate mathematical property, and identification of the name of the property. This resulted in three categories of scores: the nine property questions (NPQ) 
score, the usage of properties (UOP) score, and the naming properties (NP) score. The maximum possible points for these three types of scores was 9 .

Audio-taped interviews. Protocols (see Appendix B) for interviews were created for use in this study. Audio-taped interview data provided additional insight into participants' understanding that the paper-and-pencil assessments alone could not provide. Each interview was conducted individually and tape-recorded. The interview protocol that was used in this study was replicated and modified from that which was used in the Kanbir et al. (2018) study and in my second preliminary study (Monandi, 2019). There were three identical interview protocols: pre-teaching interview protocol for MT1 participants, post-teaching interview protocol for MT1 participants, and the interview protocol for MT2 and MT3 participants. There were minor differences between the protocols as a result of modifying some of the numerical expressions in the interview prompts. The interview protocol for MT2 and MT3 participants is presented below as an example. During the course the interview, the participant had access to a writing tool, a piece of paper, and the following items:

- A sheet of paper with $482+(18+300)$ on it. [See Question (2) in the Protocol below.]

- A sheet of paper with value of $4 \times(1 / 4 \times 128)$ on it. [See (3) below.]

- A sheet of paper with $315 \times 0.7+315 \times 0.3$ on it. [See (4) below.]

- A sheet of paper with $5.25 x=70 \times 5.25+30 \times 5.25$ on it. [See (5) below.]

- A sheet of paper with $92 \times 5$ on it. [See (6) below.]

- A sheet of paper with $13+y=14+13$ on it. [See (7) below.]

- A sheet of paper with the terms additive identity and multiplicative identity written on it. [See (8) below.]

- A sheet of paper with the terms additive inverse and multiplicative inverse written on it. [See (9) below.]

\section{Interview Prompts}

1. "I am going to say two words and, as soon as I say them, I want you to say something, or draw something, or do something - do the first thing that comes into your head after I say the words. The words are ... "distributive property." Here are the words again: "distributive property." 
2. Without using a calculator, find the value of $482+(18+300)$.

[Once an answer is given ask for the explanation of where that answer came from.] [After the student has given an explanation, ask the student to think of a different way of finding the solution.]

3. Without using a calculator, find the value of $4 \times(1 / 4 \times 128)$.

[Once an answer is given ask for the explanation of where that answer came from.]

[After the student has given explanation, ask the student to think of a different way of finding solution.]

4. Without using a calculator, what is the value of $315 \times 0.7+315 \times 0.3$ ?

[When the student gives an answer, ask her or him to write down how she or he obtained that answer. Also, ask the student to explain what she or he thought, in words. Then ask the student to think of another way of evaluating the expression]

5. Give the student a piece of paper with $5.25 x=70 \times 5.25+30 \times 5.25$ on it, then ask her or him to say which values of $x$ would make the equation true.

[When the student gives an answer, ask her or him to write down how she or he obtained that answer. Also, ask the student to explain what she or he thought, in words. Then ask the student to think of another way of finding solution.]

6. Give the student a piece of paper with $92 \times 5$ on it, then ask her or him to compute $92 \times 5$. [When the student gives an answer, ask her or him to explain how she or he obtained that answer. Also, ask the student to explain why his or her strategy for obtaining the answer works. Challenge the student to explain whether she or he is applying a mathematical property.]

7. Give the student a piece of paper with $13+y=14+13$ on it, then ask her or him to say which values of $y$ would make the equation true.

[Once an answer is given ask for the explanation of where that answer came from.]

[After the student has given explanation, ask the student to think of a different way of finding solution.]

8. "I am going to show you a sheet of paper with some words on it." [Show the pupil a piece of paper with the terms ADDITIVE IDENTITY and MULTIPLICATIVE IDENTITY.] "What do these terms mean?"

[After the pupil gives his or her answer, ask him or her whether he or she had heard of these terms before. If the pupil responded with "I don't know" to the initial question, ask him/her to say what he or she thinks the terms mean.]

9. I am going to show you a sheet of paper with some words on it. [Show the pupil a piece of paper with the terms ADDITIVE INVERSE and MULTIPLICATIVE INVERSE.] "What do these terms mean?" 
[After the pupil gives his or her answer, ask him or her whether he or she had heard of these terms before. If the pupil responded with "I don't know" to the initial question, ask him/her to say what he or she thinks the terms mean.]

\section{Data Collection Procedures}

Data was collected in fall 2019 from a convenience sample of prospective elementaryschool teachers (PETs) enrolled in mathematics courses offered at a community college in the U.S. Mid-Atlantic. All procedures and consent were followed as presented in the IRB approval forms. Potential participants were informed during recruitment that their participation in this study was voluntary and that there would be no penalty of any kind for choosing not to participate. Potential participants were also informed that they could withdraw from the study at any time even after initially agreeing to participate. This study sought participants who were over the age of 18 and who were prospective elementary-school teachers. Data were collected from MT1, MT2, and MT3 courses at the community college in the U.S. Mid-Atlantic. These data were collected from participants' written responses to a mathematics paper-and-pencil instrument and from audio-taped interviews.

The MT1 course associated with this study was taught as a face-to-face lecture class. Prospective teachers in the MT1 course completed two parallel assessment instruments on two separate occasions (at the pre-teaching and post-teaching stages) as part of their regular coursework, but the assessments did not count toward their course grade. A different faculty member served as the recruiter for participants in MT1 course because I was the instructor of the

course. There were 27 prospective teachers enrolled in MT1, but only 17 of them agreed to have their written responses on the pre-teaching and post-teaching instruments analyzed for use in this study. 
Because I was not the instructor of either the MT2 or MT3 course, I visited prospective teachers in these two courses in their respective classes for recruitment. Participants in MT2 and MT3 took one of the paper-and-pencil instruments as part of their regular coursework. Even though this assessment was taken as part of regular coursework, it did not count toward course grade. MT2 and MT3 tend to be low enrollment courses. There were two sections of MT2 running in fall 2019 - one of them was a face-to-face lecture class and the other was hybrid. MT2 participants were sought from the face-to-face lecture class because the nature of the hybrid section posed a challenge to conduct the recruitment and to reserve 50 minutes for the paper-andpencil assessment. There were 14 prospective teachers enrolled in the MT2 course included in this study, but only 6 of them completed the paper-and-pencil instrument and consented to having their responses analyzed for use in this study.

There were two sections of MT3 running in fall 2019, and they were both face-to-face lecture classes. One of the MT3 sections had 11 prospective teachers enrolled, and the other had 13 prospective teachers enrolled. Participants were sought from both sections of MT3. Nine prospective teachers from the first MT3 section and 7 prospective teachers from the second MT3 section completed the paper-and-pencil assessment and consented to having their responses analyzed for use in this study. Thus, there was a total of 16 MT3 prospective teachers who agreed to participate, and data collected from them were combined for analysis.

Assessment I (see Appendix C) was used as the pre-teaching assessment in MT1 course, and Assessment II (see Appendix C) was used as the post-teaching assessment. MT2 and MT3 prospective teachers only took one assessment-Assessment I. For the audio-taped interviews, I had planned to interview at least three students from each of the three mathematics courses. The goal was to interview at least one student with an above average score, one average-performing 
student, and one student with a below average score in each of the three mathematics courses. However, I was not successful in doing this because many prospective teachers said they had hectic class schedules, and many had jobs outside of school. Two prospective teachers from the MT1 course agreed to be interviewed, and they both participated in one-on-one pre-teaching and post-teaching interviews. One MT2 prospective teacher and one MT3 prospective teacher agreed to be interviewed. All interviews were conducted one-on-one to explore each interviewee's understanding of number property concepts.

\section{Methods for Data Analysis}

Data analysis involved both quantitative and qualitative methods. After all data collection was concluded, data from the paper-and-pencil assessments were scored and the audio-taped interviews were transcribed. Participants' written responses on the paper-and-pencil instruments were analyzed and categorized based on correctness and strategies used. The transcribed text of audio-taped interviews was analyzed by examining the strategies used by participating interviewees.

\section{Qualitative Analysis}

Qualitative analysis consisted of analyzing participants' written responses to questions on the paper-and-pencil instruments as well as analyzing participating interviewees' responses to tasks in the interview protocol. Question 1 and Question 12 on the paper-and-pencil instruments required the participants to describe a mathematical property in words. For these two questions, the participant's response was categorized as either appropriate or inappropriate. If the participant's response was deemed appropriate it was assigned a 1, and if the response was deemed inappropriate it was assigned a 0. Analyses of these questions, Question 1 and Question 
12 , included scoring participants' responses and documenting the number of responses that were appropriate, and examining the most common incorrect definitions of the mathematical properties.

There were nine questions, NPQs, on the paper-and-pencil instruments which specifically assessed whether participating PETs could recognize and use number properties appropriately to simplify expressions or solve equations, and to assess whether they could identify these properties by name. The mathematical sentences in each NPQ and corresponding property being assessed are listed in Table 1. For NPQs, analyses included examination of whether participants recognized and used number properties where appropriate and whether they correctly identified the properties by name.

\section{Table 1}

$N P Q$ Number Sentences and Corresponding Property

\begin{tabular}{lccl}
\hline \multirow{2}{*}{ Question } & \multicolumn{2}{c}{ Number Sentence } & \multicolumn{1}{c}{ Property } \\
\cline { 2 - 3 } & Assessment I (Pre-T) & Assessment II (Post-T) & \\
\hline Question 2 & $940+(60+427)$ & $920+(80+533)$ & Associative property for + \\
Question 3 & $13 \times y=9 \times 13$ & $15 \times y=5 \times 15$ & Commutative property for $\times$ \\
Question 4 & $3 x=21 \times 3+4 \times 3$ & $3 x=21 \times 3+4 \times 3$ & Distributive Property \\
Question 5 & $(72 \times 5) \times 2$ & $(36 \times 5) \times 2$ & Associative property for $\times$ \\
Question 7 & $0.6 \times 250+250 \times 0.4$ & $0.8 \times 315+315 \times 0.2$ & Distributive Property \\
& & & \\
Question 8 & $20 \times(10+5)=(20 \times$ & $50 \times(10+5)=(50 \times$ & Distributive Property \\
Question 9 & $10)+(20 \times y)$ & $10)+(50 \times y)$ & \\
Question 10 & $(120 \times(1 / 32)) \times 64$ & $48 \times((1 / 24) \times 150)$ & Associative property for $\times$ \\
Question 11 & $(1 / 5) \times 96+(1 / 5) \times 4$ & $(1 / 5) \times 96+(1 / 5) \times 4$ & Distributive Property \\
\hline
\end{tabular}


Consider the following NPQ:

Question 2. Suppose you were asked to calculate the value of $940+(60+427)$ in your head. How would you do it, and which property would you be using?

Can you think of a different way of evaluating $940+(60+427)$ ? Explain.

This question was framed with the associative property for addition in mind. The question was designed to assess whether participants recognized that the associative property for addition could be applied to find the value of $940+(60+427)$. For such a question, the participants' response is first checked for correctness on all strategies employed. Second, the participants' response is checked for strategy and whether a number property was correctly employed in at least one strategy. Third, the response is checked for whether the participant correctly named the property used. Below is a sample of an MT2 participant's response to Question 2 (see Figure 2).

2. Suppose you were asked to calculate the value of $940+(60+427)$ in your head. How would you do it, and which property would you be using?

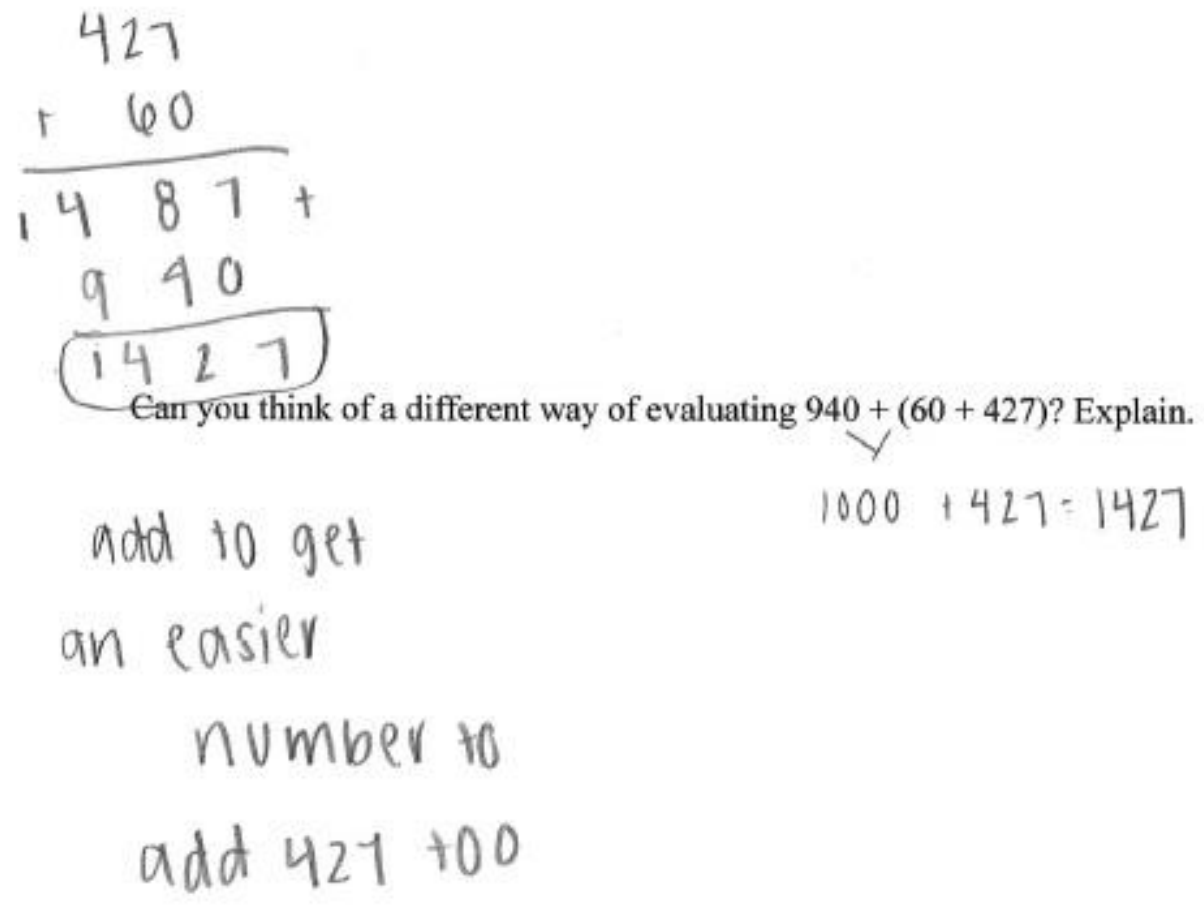

Figure 2. Sample of participant's response to Question 2. 
The MT2 participant, whose work is shown in Figure 2, appropriately responded to Question 2. No errors were observed in the strategies employed the participant employed. So, the participants' overall response to the question was coded as correct. The participant employed a number property in her second strategy. She recognized that 940 and 60 could be associated and correctly responded the problem using this approach. The participant's response was coded as correct for the aspect of applying the appropriate mathematical property. The participant, however, did not name the property used. So, for this aspect of the question, the participant's response was coded as incorrect.

For Question 3, the same MT2 participant responded as shown in Figure 3. The participant correctly responded to Question 3 and mentioned the word “commutative,' so the participant's response was coded as correct for overall response, strategy, and naming the property. Question 3 was framed with the commutative property for multiplication in mind. Even though the participant did not use the words "commutative property for multiplication," her use of "communtative" [taken to mean commutative] communicated that the participant was aware that multiplication is commutative.

3. If $13 \times y=9 \times 13$, what must $y$ equal?

Which property allows you to quickly determine the value of $y$ ?

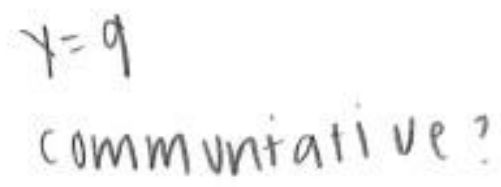

Figure 3. Sample of participant's response to Question 3.

For Question 6 and Question 13, which were included on the paper-and-pencil assessments to assess whether participating PETs would inappropriately apply a number property, analyses included examining the participants' responses and recording the number of 
responses that were correct and recording the number of responses in which a number property was inappropriately applied to an operation in which the property does not hold. Question 14 and Questions 15 on the paper-and-pencils assessments were included to assess whether participating PETs could explain using number properties the steps taken by some hypothetical students in evaluating a numerical expression. Analyses of these questions consisted of examining whether participants correctly recognized the number properties that the hypothetical students used to move from one step to the next when evaluating the given numerical expressions.

Four participating PETs agreed to participate in audio-taped interviews: two MT1 students, one MT2 student, and one MT3 student. Analysis of audio-taped interviews consisted of analyzing the transcribed text of all audio-recordings. The pre-teaching and post-teaching interviews of the participating MT1 interviewees were analyzed first. Then, analysis of interviews with participating MT2 and MT3 interviewees followed. Analysis of these interviews included examining each response to an interview prompt and ascertaining what the participating interviewees knew and understood about the various properties of real numbers. The following questions were among those considered when analyzing the audio-taped interviews. Could the participating interviews provide appropriate definitions of properties or accurately explain the meanings of properties? Did they appropriately apply properties in relevant situations? Did they recognize that in certain situations using properties is more appropriate than using the rules for order of operations?

\section{Quantitative Analysis}

As stated previously, the responses of participating students to tasks on each assessment instrument were evaluated and assigned four different scores: an overall score for the entire assessment, a score on the nine property questions, a score on the usage of properties, and a score 
on identifying properties by name. The overall score was determined by scoring all 15 questions, and the maximum possible points for the overall score was 15 . For the nine property questions, each question was scored for correctness, application of the appropriate mathematical property, and identification of the name of the property. This resulted in three categories of scores: the nine property questions (NPQ) score, the usage of properties (UOP) score, and the naming properties (NP) score. The maximum possible points for the NPQ, UOP, and NP scores was 9. Quantitative analysis not only included summary statistics of the participants' performance on the paper-and-pencil instruments but also provided a means to compare pre-teaching and postteaching performances of MT1 participants as well as a way to compare MT1 participants' performance to that of MT2/MT3 participants. Because the college allows PETs to be concurrently enrolled MT2 and MT3, MT2 and MT3 data were combined and studied together as one MT2/MT3 group. MT2/MT3 PETs were expected to have already completed and passed the MT1 course.

Repeated-measures $t$-tests and independent-samples $t$-tests were used as tools to make comparisons between the groups in the study. Because this study did not involve random samples, these statistical tests were not used to draw general conclusions about population parameters and instead were only used as a means to compare the performance on the paper-andpencil assessments between the groups in the study. Repeated-measures $t$-tests were used to compare the pre-teaching and post-teaching performance of MT1 participants. To augment the repeated-measures t-tests, Cohen's $d$ effect sizes (Cohen, 1988) and performance improvement ratios (PIRs) were calculated. Independent-samples $t$-tests were used to compare the performance between the performance of MT1 participants (both pre-teaching and post-teaching) and the performance of MT2/MT3 participants. 
Repeated-measures $t$-tests. One of the aims of this study was to compare the PETs' MT1 pre-teaching and post-teaching mean performances. Repeated-measures t-tests, with $\alpha=$ 0.05 level of significance, were conducted to test for differences in the mean performance of MT1 PETs on the pre-teaching and post-teaching assessments. The first repeated-measures $t$-test was conducted to test whether there was a statistically significant difference in the overall score of the PETs on the pre-teaching and post-teaching MT1 assessments. The null hypothesis was that: the mean pre-teaching and post-teaching overall scores would not be statistically significantly different. That is to say, if the null and research hypotheses are denoted by $\mathrm{H}_{0}$ and $\mathrm{H}_{1}$, then:

$\mathrm{H}_{0}$ : The difference between the mean of the pre-teaching overall scores and the mean of the post-teaching overall scores would be equal to zero.

$\mathrm{H}_{1}$ : The difference between the mean of the pre-teaching overall scores and the mean of the post-teaching overall scores would not be equal to zero.

The second repeated-measures $t$-test was conducted to test whether the scores of PETs on the nine property MT1 questions (NPQs) differed statistically significantly between the preteaching and post-teaching assessments. The null hypothesis was that the mean pre-teaching and post-teaching NPQ scores were not statistically significantly different. The null and research hypotheses were:

$\mathrm{H}_{0}$ : The difference between the mean of the pre-teaching NPQ scores and the mean of the post-teaching NPQ scores would be equal to zero.

$\mathrm{H}_{1}$ : The difference between the mean of the pre-teaching NPQ scores and the mean of the post-teaching NPQ scores would not be equal to zero. 
The third repeated-measures $t$-test was conducted to test whether there was a statistically significant difference in the usage of properties (UOP) score between the pre-teaching and postteaching mean scores of the MT1 PETs students. The following null hypothesis was considered for this test: the mean pre-teaching and post-teaching UOP scores would not be statistically significantly different. Accordingly, the null and alternative hypotheses were:

$\mathrm{H}_{0}$ : The difference between the mean of the pre-teaching UOP scores and the mean of the post-teaching UOP scores would be equal to zero.

$\mathrm{H}_{1}$ : The difference between the mean of the pre-teaching UOP scores and the mean of the post-teaching UOP scores would not be equal to zero.

The fourth repeated-measures t-test was conducted to test whether there was a statistically significant difference in the naming-properties (NP) score between the pre-teaching and post-teaching assessments completed by MT1 PETs. The following null hypothesis was considered for this test: the pre-teaching and post-teaching NP scores were not statistically significantly different. Accordingly, the null and alternative hypotheses are:

$\mathrm{H}_{0}$ : The difference between the mean of the pre-teaching NP scores and the mean of the post-teaching NP scores would be equal to zero.

$\mathrm{H}_{1}$ : The difference between the mean of the pre-teaching NP scores and the mean of the post-teaching NP scores would not be equal to zero.

Cohen's $\boldsymbol{d}$ effect sizes and Performance Improvement Ratios. Because statistical significance does not mean practical significance (Coe, 2002; Fan, 2001; Sullivan \& Feinn, 2012), Cohen's $d$ effect sizes (Cohen, 1988) and performance improvement ratios (PIRs) were also calculated. Cohen's $d$, which is calculated by subtracting the mean of the post-teaching scores from the mean of the pre-teaching scores and then dividing the difference by the pooled 
standard deviation, is one of the most common measure for quantifying effect size (Coe, 2002; Fan, 2001; Sullivan \& Feinn, 2012).

$$
d=\frac{\text { Mean }_{\text {post }}-\text { Mean }_{\text {pre }}}{\sqrt{\left(\frac{S D_{\text {post }}{ }^{2}}{2}+\frac{S D_{\text {pre }}{ }^{2}}{2}\right)}}
$$

The following conventions are used to interpret Cohen's $d$ effect size (Cohen, 1988): an effect size of $d=0.2$ would be considered as "small," an effect size of $d=0.5$ would be considered as "medium," and an effect size of $d=0.8$ or higher would be considered as "large."

With a small sample size, it is possible to get a large effect size without any practical significance to the effect size (Sullivan \& Feinn, 2012; Vaiyavutjamai, 2003). So, in addition to calculating Cohen's $d$ effect sizes, performance improvement ratios were also calculated as an additional measure for quantifying the effect of teaching in MT1. Duarte (2010) described the performance improvement ratio (PIR) as the "ratio of the difference between post-teaching and pre-teaching mean scores to the difference of maximum possible test score and pre-teaching mean score" (p. 216).

$$
P I R=\frac{\text { Mean }_{\text {post }}-\text { Mean }_{\text {pre }}}{\text { Max Score }- \text { Mean }_{\text {pre }}}
$$

Cohen's $d$ effect sizes and performance improvement ratios (PIRs) were calculated for each of the four scores: Overall score, NPQ score, UOP score, and NP score.

Independent-samples $t$-tests. Another aspect of this study was to compare MT1 PETs' performance to that of MT2/MT3 PETs. First, the pre-teaching performance of MT1 PETs was compared to the assessment performance of MT2/MT3 PETs. Independent-samples $t$-tests, with $\alpha=0.05$ level of significance, were used to test for mean score differences, for all scores (Overall, NPQ, UOP, and NP), between MT1 pre-teaching performance and MT2/MT3 
assessment performance. Second, the post-teaching performance of MT1 PETs was compared to the assessment performance of MT2/MT3 PETs. Independent-samples $t$-tests, with $\alpha=0.05$ level of significance, were again employed to test for mean score differences, for all scores (Overall, NPQ, UOP, and NP), between MT1 post-teaching performance and MT2/MT3 assessment performance. Levene's test was conducted to test for homogeneity of variances. If Levene's test revealed that homogeneity of variances was not met, the Welch-Satterthwaite approximation (Satterthwaite, 1946; Welch, 1947) was employed instead. For unequal sample sizes and unequal variances, the Welch-Satterthwaite approximation has greater robustness (Derrick, Toher, \& White, 2016).

Cohen's $\boldsymbol{d}$ and $\boldsymbol{r}^{2}$ as measures of effect size. For every independent-samples $t$-test that was conducted, Cohen's $d$ and $r^{2}$ were also calculated as measures of effect size. The calculation for Cohen's $d$ effect size for independent-samples $t$-test is given below:

$$
d=\frac{\text { Mean }_{1}-\text { Mean }_{2}}{\sqrt{\left(\frac{S D_{1}{ }^{2}}{2}+\frac{S D_{2}{ }^{2}}{2}\right)}}
$$

The interpretation of Cohen's $d$ was discussed above. In addition to calculating Cohen's d effect sizes, $r^{2}$ effect sizes were calculated as an additional measure of the magnitude of effect between groups. The formula for calculating $r^{2}$ is given below:

$$
r^{2}=\frac{t^{2}}{t^{2}+d f}, \text { where } d f \text { equals the degrees of freedom. }
$$

The following conventions are used to interpret $r^{2}$ : an effect size of $r^{2}=0.01$ would be considered as "small," an effect size of $r^{2}=0.09$ would be considered as "medium," and an effect size of $r^{2}=0.25$ would be considered as "large." It is important to note that small sample 
sizes may yield large effect sizes without any practical significance (Sullivan \& Feinn, 2012; Vaiyavutjamai, 2003).

\section{Summary}

This chapter has provided details of the research methods used in this dissertation study. This dissertation study is a mixed-methods investigation utilizing both quantitative and qualitative methods for data analysis. Data were collected from administered paper-and-pencil assessments and audio-taped interviews. The next chapter will report on results of this study's data analysis. In Chapter 4, the results of the qualitative analysis of data collected from participants' written responses on the paper-and-pencil assessments and from audio-taped interviews are also reported. Also in Chapter 4, results of the repeated-measures $t$-tests and of the independent-samples $t$-tests are presented. 


\section{CHAPTER IV: RESULTS}

Analysis of data involved the use of both quantitative and qualitative techniques for data analysis. The data collected in this dissertation study shed light on prospective teachers' knowledge of number properties. It was decided that a combination of qualitative and quantitative approaches to data analysis would be incorporated in the study. Glesne (1999) noted that qualitative and quantitative methodologies could lead to very different findings but also have the potential to complement each other. In this dissertation study, quantitative analysis not only included summary statistics of the participants' performance on the paper-and-pencil assessment instruments but also provided a means to compare pre-teaching and post-teaching performance of MT1 participants as well as a way to compare MT1 participants' performance to those of MT2 and MT3 participants.

Qualitative analysis techniques were employed to analyze the transcribed audio-taped interviews and participants' written responses to questions on the paper-and-pencil assessments. Theories on cognitive learning structures that linked Peirce's concepts of "signifier," "interpretant," and "object" were central to the interpretation of this study's data. From the Peircean perspective, the following question can be taken as a sign because it was framed with a certain mathematical object in mind - the commutative property for addition: "If $16+26=x+$ 16 , what must $x$ equal? Which property allows you to determine quickly the value of $x$ ?" The same sign may signify different mathematical objects to different students. It is possible for students to see this sign but not respond in a manner that expressively demonstrates that they recognize the sign. Kanbir et al. (2018) wrote, "with respect to any concept it is likely that different students will have different 'cognitive structures,' and that fact will result in their interpreting the signs in different ways" (p. 103). 


\section{Qualitative Results}

There were 15 questions on the paper-and-pencil instruments. The following abbreviations for questions on the paper-and-pencil instruments are used: Q1 for Question 1, Q2 for Question 2, Q3 for Question 3, and so forth. Two questions-Q1 and Q12—required the participants to describe a mathematical property in words. Q6 and Q13 were included to assess whether participating PETs would inappropriately apply a number property to an operation in which the property does not hold.

\section{Table 2}

Nine Questions on Using Number Properties

\begin{tabular}{|c|c|c|}
\hline Q\# & Assessment I (Pre-Teaching) & Assessment II (Post-Teaching) \\
\hline Q2 & $\begin{array}{l}\text { Find the value of } \\
\qquad 940+(60+427)\end{array}$ & $\begin{array}{l}\text { Find the value of } \\
\qquad 920+(80+533)\end{array}$ \\
\hline Q3 & $\begin{array}{l}\text { Find the value of } y \text { : } \\
\qquad 13 \times y=9 \times 13\end{array}$ & $\begin{array}{l}\text { Find the value of } y \text { : } \\
\qquad 15 \times y=5 \times 15\end{array}$ \\
\hline Q4 & $\begin{array}{l}\text { Find the value of } x \text { : } \\
\qquad 3 x=21 \times 3+4 \times 3\end{array}$ & $\begin{array}{l}\text { Find the value of } x \text { : } \\
\qquad 3 x=21 \times 3+4 \times 3\end{array}$ \\
\hline Q5 & $\begin{array}{l}\text { Find the value of } \\
\qquad(72 \times 5) \times 2\end{array}$ & $\begin{array}{l}\text { Find the value of } \\
\qquad(36 \times 5) \times 2\end{array}$ \\
\hline Q7 & $\begin{array}{l}\text { Find the value of } \\
\qquad 0.6 \times 250+250 \times 0.4\end{array}$ & $\begin{array}{l}\text { Find the value of } \\
\qquad 0.8 \times 315+315 \times 0.2\end{array}$ \\
\hline Q8 & $\begin{array}{l}\text { Find the value of } y \text { : } \\
\qquad 20 \times(10+5)=(20 \times 10)+(20 \times y)\end{array}$ & $\begin{array}{l}\text { Find the value of } y \text { : } \\
50 \times(10+5)=(50 \times 10)+(50 \times y)\end{array}$ \\
\hline Q9 & $\begin{array}{l}\text { Find the value of } x \text { : } \\
\qquad 16+26=x+16\end{array}$ & $\begin{array}{l}\text { Find the value of } x \text { : } \\
\qquad 36+18=x+36\end{array}$ \\
\hline Q10 & $\begin{array}{l}\text { Find the value of } \\
\qquad(120 \times(1 / 32)) \times 64\end{array}$ & $\begin{array}{l}\text { Find the value of } \\
\qquad 48 \times((1 / 24) \times 150)\end{array}$ \\
\hline Q11 & $\begin{array}{l}\text { Find the value of } \\
\qquad(1 / 5) \times 96+(1 / 5) \times 4\end{array}$ & $\begin{array}{l}\text { Find the value of } \\
\qquad(1 / 5) \times 96+(1 / 5) \times 4\end{array}$ \\
\hline
\end{tabular}

Note. The nine questions on each paper-and-pencil instrument were such that number properties could be applied.

Nine questions, shown in Table 2 in abbreviated format, specifically assessed whether participating PETs could recognize and use number properties appropriately to simplify 
expressions or solve equations, and to assess whether they could identify these properties by name. From the Peircean perspective, these nine property questions (NPQs) could be taken as signs because they were framed with certain mathematical objects in mind. Q14 and Q15 were included to assess whether participating PETs could explain using number properties the steps taken by some hypothetical students in evaluating a numerical expression.

\section{MT1 Participants' Knowledge of Properties}

A major aspect of this study was to ascertain what participating MT1 PETs knew and understood about properties of real numbers before and after participating in instruction emphasizing number properties. MT1 participants' written responses on the pre-teaching and post-teaching instruments were analyzed, and the results of this analysis are discussed in this section. Seventeen MT1 PETs agreed to participate in this dissertation study. However, only 16 of them were present to respond to the pre-teaching instrument and 16 were again present to respond to the post-teaching instrument. So, what knowledge did participating MT1 PETs exhibit on the pre-teaching and post-teaching instruments regarding each of the number properties?

Commutative property for addition. Q9 was included on both the pre-teaching and post-teaching instruments. Q9 can be taken as a sign because it was framed with a certain mathematical object in mind - the commutative property for addition: "If $16+26=x+16$, what must $x$ equal? Which property allows you to determine quickly the value of $x$ ?" On the preteaching instrument, 15 out of 16 MT1 participants correctly determined the value of $x$ in $16+26$ $=x+16$, but none of them responded in a manner that demonstrated that they understood the mathematical object behind the question. The commutative property for addition was not used or mentioned by any of the 16 participants. The most common response involved isolating the variable by subtracting 16 from both sides of the equation or "canceling" 16 from both sides of 
the equal sign. Those who responded in this manner had seen the sign but failed to respond in a manner that demonstrated that they recognized the mathematical object behind the question, which was the commutative property for addition. One of the MT1 participants correctly answered this question but stated that the property applied was the reflexive property.

\section{Table 3}

Pre-T and Post-T Responses to Commutative Property Questions

\begin{tabular}{|c|c|c|c|c|c|c|}
\hline Property & Q\# & & & $\begin{array}{l}\text { No. of Correct } \\
\text { Responses }(\%)\end{array}$ & $\begin{array}{l}\text { No. Relying on } \\
\text { Property (\%) }\end{array}$ & $\begin{array}{l}\text { No. Naming } \\
\text { Property }(\%)\end{array}$ \\
\hline Commutative & \multirow{2}{*}{ Q9 } & Pre-t & $16+26=x+16$ & $15 \quad(94 \%)$ & $0 \quad(0 \%)$ & $0 \quad(0 \%)$ \\
\hline Property for + & & Post-t & $36+18=x+36$ & $15 \quad(94 \%)$ & $6 \quad(38 \%)$ & $7 \quad(44 \%)$ \\
\hline Commutative & \multirow{2}{*}{ Q1 } & Pre-t & Describe Property & $(13 \%)$ & -------------- & -------------- \\
\hline Property for $x$ & & Post-t & Describe Property & $7 \quad(44 \%)$ & ------------ & -------------- \\
\hline Commutative & \multirow{2}{*}{ Q3 } & Pre-t & $13 \times y=9 \times 13$ & $13 \quad(81 \%)$ & $0 \quad(0 \%)$ & $0 \quad(0 \%)$ \\
\hline Property for $x$ & & Post-t & $15 \times y=5 \times 15$ & $15 \quad(94 \%)$ & $6 \quad(38 \%)$ & $6 \quad(38 \%)$ \\
\hline
\end{tabular}

On the post-teaching instrument, there were 15 out of 16 MT1 participants who correctly stated the value for $x$. However, only 6 of those participants used the words "commutative property" or "commutative property for addition." These 6 participants recognized the sign and responded in way that showed that they recognized the mathematical object under consideration was the commutative property for addition. There was one participant who used the words “commutative property for addition" but did not state the value for $x$. There were five participants correctly stated the value of $x$ but misidentified the property used. One of the participants wrote that the property used was the additive property. Another participant wrote that the property used was the "deductive property." Three other participants wrote that the property used was the associative property. All the other MT1 participants correctly stated the value for $x$ but did not use or mention the commutative property for addition. 
Commutative property for multiplication. Q1 was included on the pre-teaching and post-teaching instruments to assess whether participating MT1 PETs could accurately describe the meaning of the commutative property for multiplication. Out of 16 participating MT1 PETs, only 2 of them correctly responded to $\mathrm{Q} 1$ on the pre-teaching instrument. On the post-teaching instrument, 7 out of 16 participating MT1 PETs correctly responded to Q1. In Figure 4, the student's concept image of the commutative property for multiplication improved between the pre-teaching and post-teaching stages. On the post-teaching instrument, there were four participants who incorrectly described the associative property for multiplication instead. One participant described the commutative property for addition instead of describing the one for multiplication.

\section{Pre-Teaching}

\section{Task: Describe the commutative property for multiplication}

A really important property for numbers and for algebra is called the commutative property for multiplication. Describe this property.

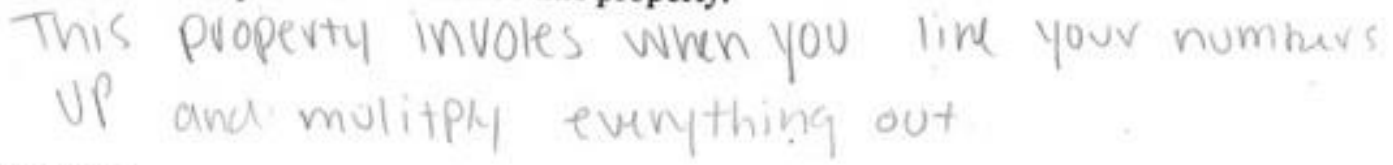

\section{Post-Teaching}

A really important property for numbers and for algebra is called the commutative property for multiplication. Describe this property.

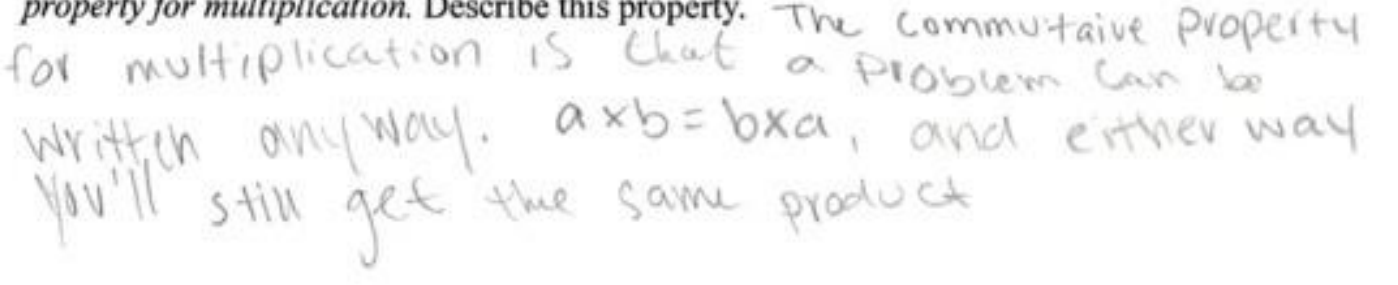

Figure 4. Change in a student's concept image of the commutative property for multiplication.

Q3 was included to assess whether participating MT1 PETs recognized role of the commutative property for multiplication in determining quickly the value of $y$ in the following equations: $13 \times y=9 \times 13$ and $15 \times y=5 \times 15$. On the pre-teaching instrument, there were 13 correct responses to Q3. None of the MT1 PETs responded in a way that demonstrated that they 
recognized the mathematical object under consideration. None of them used the words "commutative property" or "commutative property for multiplication." The most common explanation given was dividing both sides of the equation by 13 to isolate the variable $y$.

On the post-teaching instrument, there were 15 out of 16 MT1 participants who correctly stated the value for $y$. Only 6 of those participants used the words "commutative property" or “commutative property for multiplication." These 6 participants recognized the sign and responded in way that showed that they knew the mathematical object under consideration was the commutative property for multiplication. One participant correctly stated the value for $y$ but wrote the words "multiplicative identity." Another participant wrote the words "inductive property." These two participants did not respond in way that showed that they knew the correct terminology for the mathematical object behind the sign. In most of the other responses, explanations were not given. Because of this and because we cannot know what was going on in the participants' mind, it was not possible to ascertain confidently whether the rest of the participants recognized the mathematical object behind the sign.

Associative property for addition. Q2 and Q12 were included on the pre-teaching and post-teaching instruments to assess what participating MT1 PETs knew about the associative property for addition. For Q12, participants were asked to describe the meaning of the associative property for addition. None of the 16 participating MT1 PETs correctly responded to Q12 at the pre-teaching stage. At the post-teaching stage, 5 of the 16 participating MT1 PETs correctly responded to Q12; six MT1 PETs described the commutative property for addition instead.

For Q2 on the pre-teaching instrument, participants were asked to find the value of $940+$ $(60+427)$ using two different strategies. For this question, participants who initially attempted 
to find the sum of 60 and 427 failed to respond appropriately to the sign for which the mathematical object under consideration was the associative property for addition. There were 9 correct responses with two unique strategies and 5 correct responses with only one unique strategy. The use of order of operations was the most common strategy in all responses; order of operations was used as a strategy in 12 of the 14 correct responses. Five out of 16 MT1 participants associated 940 and 60 in at least one of their strategies. None of them used the words "associative property" or "associative property for addition," except for one who wrote, "Maybe associative?" Two participants incorrectly responded to Q2, and they both attempted to find the answer to Q2 by using order of operations.

\section{Table 4}

Pre-T and Post-T Responses to Associative Property Questions

\begin{tabular}{|c|c|c|c|c|c|c|}
\hline Property & Q\# & & & $\begin{array}{l}\text { No. of Correct } \\
\text { Responses (\%) }\end{array}$ & $\begin{array}{l}\text { No. Relying on } \\
\text { Property (\%) }\end{array}$ & $\begin{array}{l}\text { No. Naming } \\
\text { Property }(\%)\end{array}$ \\
\hline Associative & \multirow{2}{*}{ Q12 } & Pre-t & Describe Property & $(0 \%)$ & -------------- & -------------- \\
\hline Property for + & & Post-t & Describe Property & $(31 \%)$ & --------------- & 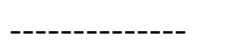 \\
\hline Associative & \multirow{2}{*}{ Q2 } & Pre-t & $940+(60+427)$ & $(88 \%)$ & $\begin{array}{ll}5 & (31 \%)\end{array}$ & $1 \quad(6 \%)$ \\
\hline Property for + & & Post-t & $920+(80+533)$ & $14 \quad(88 \%)$ & $11 \quad(69 \%)$ & $3 \quad(19 \%)$ \\
\hline \multirow{4}{*}{$\begin{array}{c}\text { Associative } \\
\text { Property for } \times\end{array}$} & \multirow{2}{*}{ Q5 } & Pre-t & $(72 \times 5) \times 2$ & $15 \quad(94 \%)$ & $(19 \%)$ & $1 \quad(6 \%)$ \\
\hline & & Post-t & $(36 \times 5) \times 2$ & $14 \quad(88 \%)$ & $10 \quad(63 \%)$ & $5 \quad(31 \%)$ \\
\hline & \multirow{2}{*}{ Q10 } & Pre-t & $(120 \times(1 / 32)) \times 64$ & $(13 \%)$ & $(13 \%)$ & $1(6 \%)$ \\
\hline & & Post-t & $48 \times((1 / 24) \times 150)$ & $(31 \%)$ & $(31 \%)$ & $2(13 \%)$ \\
\hline
\end{tabular}

For Q2 on the post-teaching instrument, participants were asked to find the value of 920 $+(80+533)$ using two different strategies. There were 12 correct responses with two unique strategies and 2 correct responses with only one unique strategy. Eleven out of 16 MT1 participants associated 920 and 80 in at least one of their strategies. Three of them used the 
words "associative property" or "associative property for addition." Four of them incorrectly referred to the property used as the "commutative property" or "commutative property for addition." More participants on the post-teaching assessment than on the pre-teaching assessment recognized and appropriately responded to the sign in a way which showed that they aware of the mathematical object under consideration. There were 2 incorrect responses to Q2, and the use of order of operations was employed in both responses.

Associative property for multiplication. Q5 and Q10 were included on the pre-teaching and post-teaching instruments to assess what participating MT1 PETs knew about the associative property for multiplication. For Q5 on the pre-teaching instrument, participants were asked to find the value of $(72 \times 5) \times 2$ using two different strategies. For this question, there were 3 correct responses with two unique strategies and 12 correct responses with only one unique strategy. The use of order of operations was the most common strategy and was present in all 15 correct responses. Three out of $16 \mathrm{MT} 1$ participants correctly recognized and responded to this sign by first computing $5 \times 2$. Only one of them used the words "associative property." There was one incorrect response. The participant who incorrectly responded to Q5 inappropriately applied the distributive property.

For Q5 on the post-teaching instrument, participants were asked to find the value of $(36 \times$ 5) $\times 2$ using two different strategies. For this question, there were 9 correct responses using two unique strategies and 5 correct responses with only one unique strategy. The use of the associative property for multiplication was present as a strategy in 10 of the 14 correct responses. Five participants used the words "associative property" or "associative property for multiplication." Three participants inappropriately used the words "commutative property" or "commutative property for multiplication." More participants relied on the associative property 
for multiplication on the post-teaching instrument than did on the pre-teaching instrument. There were 2 incorrect responses to Q5 and in both responses the use of order of operations was employed.

For Q10 on the pre-teaching instrument, participants were asked to find the value of $\left(120 \times \frac{1}{32}\right) \times 64$. Only 2 participants correctly responded to this question at the pre-teaching stage, and they both relied on the associative property for multiplication. Only one of these participants identified the associative property by name. Eight participants left this question unanswered. The remaining participants attempted but did not complete Q10. The most common strategy among those who attempted Q10, but did not complete it or answer it incorrectly, was the use of order of operations.

For Q10 on the post-teaching instrument, participants were asked to find the value of $48 \times\left(\frac{1}{24} \times 150\right)$. Five out of 16 MT1 participants recognized and correctly responded to this sign by relying on the associative property for multiplication. Two participants correctly identified the associative property for multiplication by name. One participant misidentified the property used as the "commutative property," and another misidentified it as the "additive identity property." Some of the participating MT1 PETs relied on the associative property for multiplication when they computed $(36 \times 5) \times 2$ but did not also do the same when they computed $48 \times\left(\frac{1}{24} \times 150\right)$. Most of the remaining participants left this question unanswered or did not complete it.

Distributive property. There were four questions on the pre-teaching and post-teaching instruments which were included to assess what participating MT1 PETs knew about the distributive property: Q4, Q7, Q8, and Q11. For Q4 on the pre-teaching instrument, one out of 16 
MT1 participants correctly recognized and responded to the sign by first factoring the right-hand side to yield $3 x=3 \times(21+4)$. However, this participant did not identify the property used by name. On the post-teaching instrument, none of the MT1 participants responded in a manner that showed they understood the mathematical object under consideration; none of the participants used, or attempted to use, the distributive property on the post-teaching assessment. The strategy used in nearly all of the responses was to apply the order of operations to simplify the right-hand side of the equation and then isolating the variable, or attempting to isolate the variable, by dividing by 3 into both sides of the equation.

\section{Table 5}

Pre-T and Post-T Responses to Distributive Property Questions

\begin{tabular}{|c|c|c|c|c|c|c|}
\hline Property & Q\# & & & $\begin{array}{l}\text { No. of Correct } \\
\text { Responses }(\%)\end{array}$ & $\begin{array}{l}\text { No. Relying on } \\
\text { Property (\%) }\end{array}$ & $\begin{array}{l}\text { No. Naming } \\
\text { Property }(\%)\end{array}$ \\
\hline \multirow{10}{*}{$\begin{array}{c}\text { Distributive } \\
\text { Property }\end{array}$} & \multirow{2}{*}{ Q4 } & Pre-t & $3 x=21 \times 3+4 \times 3$ & $9 \quad(56 \%)$ & $1 \quad(6 \%)$ & $0 \quad(0 \%)$ \\
\hline & & Post-t & $3 x=21 \times 3+4 \times 3$ & $10 \quad(63 \%)$ & $0 \quad(0 \%)$ & $0 \quad(0 \%)$ \\
\hline & \multirow{2}{*}{ Q7 } & Pre-t & $0.6 \times 250+250 \times 0.4$ & $\begin{array}{ll}5 & (31 \%)\end{array}$ & $0 \quad(0 \%)$ & $0 \quad(0 \%)$ \\
\hline & & Post-t & $0.8 \times 315+315 \times 0.2$ & $2(13 \%)$ & $2 \quad(13 \%)$ & $0 \quad(0 \%)$ \\
\hline & \multirow{4}{*}{ Q8 } & Pre-t & $20 \times(10+5)$ & $(6007)$ & & \\
\hline & & & $=(20 \times 10)+(20 \times y)$ & (05\%) & $1 \quad(070)$ & $(070)$ \\
\hline & & Post-t & $50 \times(10+5)$ & $(60 \%)$ & $(38 \%)$ & $(100 \%)$ \\
\hline & & & $=(50 \times 10)+(50 \times y)$ & & & \\
\hline & \multirow{2}{*}{ Q11 } & Pre-t & $(1 / 5) \times 96+(1 / 5) \times 4$ & $0 \quad(0 \%)$ & $0 \quad(0 \%)$ & $0 \quad(0 \%)$ \\
\hline & & Post-t & $(1 / 5) \times 96+(1 / 5) \times 4$ & $1 \quad(6 \%)$ & $0 \quad(0 \%)$ & $0 \quad(0 \%)$ \\
\hline
\end{tabular}

For Q7 on the pre-teaching instrument, none of the MT1 participants responded in a manner which indicated that they understood the mathematical object under consideration; none of them used, or attempted to use, the distributive property. The use of order of operations was the most common strategy in all the responses. Q7 could be simplified more readily by 
recognizing that the distributive property could be applied by factoring 250 , yielding $250 \times(0.6$ $+0.4)$. None of the participants responded in a manner which indicated that they understood that the distributive property could be applied in simplifying this manner.

For Q7 on the post-teaching instrument, Only 2 out of 16 MT1 participants recognized and appropriately responded to the sign by factoring 315 , yielding $315 \times(0.8+0.2)=315 \times 1=$ 315. However, neither of those 2 PETs included the words "distributive property" in their explanations. None of the other participants responded in a manner that suggested that they recognized the mathematical object under consideration; none of them used, or attempted to use, the distributive property. Four of the participants inappropriately rewrote $0.8 \times 315+315 \times 0.2$ as $(315+315) \times(0.8+0.2)$.

For Q8 on the pre-teaching instrument, participants were asked to find the value of $y$ in $20 \times(10+5)=(20 \times 10)+(20 \times y)$. There are 10 correct responses to this question. Only one of the participants applied the distributive property to determine the value of $y$. However, this participant did not explicitly state that she was using the distributive property. The most common explanation given for Q8 was isolating the variable by first simplifying both sides of the equation as needed using order of operations. For Q8 on the post-teaching instrument, participants were asked to find the value of $y$ in $50 \times(10+5)=(50 \times 10)+(50 \times y)$. There were 11 correct responses to this question. Six participants applied the distributive property to determine the value of $y$. Three of them correctly identified the property by name. Even at the post-teaching stage, the most common strategy used was order of operations to isolate the variable by first simplifying each side of the equation as needed.

For Q11 on both the pre-teaching and post-teaching instruments, participants were asked to find the value of $\frac{1}{5} \times 96+\frac{1}{5} \times 4$. Q11 could be readily evaluated by relying on the distributive 
property to factor and evaluate as follows: $\frac{1}{5} \times 96+\frac{1}{5} \times 4=\frac{1}{5} \times(96+4)=\frac{1}{5} \times 100=20$.

None of the participants answered this question correctly at the pre-teaching stage. Most of the participants left this question unanswered. At the post-teaching stage, only one of the 16 participants answered this question correctly. This participant correctly relied on the distributive property but incorrectly stated that the property used was the associative property. Most of the participants at the post-teaching stage left this question unanswered or did not complete it.

\section{MT2/MT3 Participants' Knowledge of Properties}

Another major aspect of this study was to ascertain what participating MT2/MT3 PETs knew and understood about properties of real numbers. MT2/MT3 PETs did not participate in instruction emphasizing number properties because such instruction should have been received when they took the MT1 course. Thus, MT2/MT3 participants only completed one paper-andpencil instrument. There were 6 MT2 participants and 16 MT3 participants. The assessment completed by the MT2/MT3 participants was the same as that completed by the MT1 participants at the pre-teaching stage. The results of MT2 and MT3 participants on the paperand-pencil instrument were combined and studied as one to learn what they knew regarding each of the number properties.

Commutative property for addition. MT2/MT3 participants were shown the following sign on their paper-and-pencil instrument: "If $16+26=x+16$, what must $x$ equal? Which property allows you quickly to determine the value of $x$ ?" Four out of 6 MT2 participants correctly stated the value for $x$, but only one of them responded in a way which indicated that he (or she) recognized the mathematical object behind the question; only one participant used the words "commutative property." Two MT2 participants correctly stated the value of $x$, but one misidentified the property used as the "associative property" and another misidentified it as 
“transitive." Another MT2 participant who did not state the correct value for $x$ used was words “transitive property." Fifteen out of 16 MT3 participants correctly stated the value for $x$, but only 5 of them responded in a way which indicated they recognized the mathematical object behind the question; four of them used the words "commutative property," and one explained that the value for $x=26$ "because $a+b=b+a$." One of the MT3 participants correctly stated the value for $x$ but wrote, "I don't remember the name of the property." Many of the remaining MT3 participants stated the value for $x$ without writing an explanation or naming a property.

\section{Table 6}

MT2/MT3 Responses to Commutative Property Questions

\begin{tabular}{cccccc}
\hline Property & Q\# & & $\begin{array}{c}\text { No. of } \\
\text { Correct } \\
\text { Responses } \\
(\%)\end{array}$ & $\begin{array}{c}\text { No. Relying } \\
\text { on Property } \\
(\%)\end{array}$ & $\begin{array}{c}\text { No. } \\
\text { Naming } \\
\text { Property } \\
(\%)\end{array}$ \\
\hline $\begin{array}{c}\text { Commutative } \\
\text { Property for + }\end{array}$ & Q9 & $16+26=x+16$ & $19(86 \%)$ & $6(27 \%)$ & $5(23 \%)$ \\
\hline $\begin{array}{c}\text { Commutative } \\
\text { Property for } \times\end{array}$ & Q1 & Describe Property & $5(23 \%)$ & ------------- & ------------ \\
\hline $\begin{array}{c}\text { Commutative } \\
\text { Property for } \times\end{array}$ & Q3 & $13 \times y=9 \times 13$ & $19(86 \%)$ & $6(27 \%)$ & $6(27 \%)$ \\
\hline
\end{tabular}

Commutative property for multiplication. For Q1, MT2/MT3 participants were expected to describe the meaning of the commutative property for multiplication. Only one of the 6 participating MT2 PETs correctly responded to Q1, and among 16 participating MT3 PETs only 4 of them correctly responded to Q1. For Q3, MT2/MT3 participants were expected to identify the property which allows them to determine quickly the value of $y$ in the following equation: $13 \times y=9 \times 13$. All 6 MT2 participants correctly stated the value for $y$, but only 3 of them responded in a way which demonstrated that they recognized the mathematical object 
under consideration. One of the MT2 participants used the words "associative property," and another wrote "reflexive property." Thirteen of the 16 MT3 participants correctly stated the value for $y$, but only 3 of them responded in a way which demonstrated they recognized the mathematical object under consideration; three participants used the words "commutative property."

Associative property for addition. Q2 and Q12 were included to assess what participating MT1 PETs knew about the associative property for addition. For Q12, MT2/MT3 participants were expected to describe the meaning of the associative property for addition. Only one of the 6 participating MT2 PETs correctly responded to Q12, and none of 16 participating MT3 PETs correctly responded to Q12.

\section{Table 7}

MT2/MT3 Responses to Associative Property Questions

\begin{tabular}{|c|c|c|c|c|c|}
\hline Property & Q\# & & $\begin{array}{c}\text { No. of } \\
\text { Correct } \\
\text { Responses } \\
(\%)\end{array}$ & $\begin{array}{c}\text { No. Relying } \\
\text { on Property } \\
(\%)\end{array}$ & $\begin{array}{c}\text { No. } \\
\text { Naming } \\
\text { Property } \\
(\%)\end{array}$ \\
\hline $\begin{array}{c}\text { Associative } \\
\text { Property for + }\end{array}$ & Q12 & Describe Property & $1(5 \%)$ & --------------- & $\begin{array}{c}---------- \\
-\end{array}$ \\
\hline $\begin{array}{c}\text { Associative } \\
\text { Property for }+\end{array}$ & Q2 & $940+(60+427)$ & $14(64 \%)$ & $10(45 \%)$ & $1(5 \%)$ \\
\hline Associative & Q5 & $(72 \times 5) \times 2$ & $18(82 \%)$ & $8(36 \%)$ & $1(5 \%)$ \\
\hline Property for $x$ & Q10 & $(120 \times(1 / 32)) \times 64$ & $3(14 \%)$ & $3(14 \%)$ & $0(0 \%)$ \\
\hline
\end{tabular}

For Q2, MT2/MT3 participants were asked to find the value of $940+(60+427)$ using two different strategies. Four out of 6 MT2 participants recognized and appropriately responded to this sign by associating 940 and 60 in at least one of their strategies; one of them used the 
words "associative property," and another associated 940 and 60 but wrote the words “commutative property." Two of the 16 MT2 participants simplified $940+(60+427)$ by using only order of operations. These two participants saw the sign but did not recognize it because they did not respond in a way which showed they recognized that the mathematical object under consideration was the associative property for addition.

Among the 16 MT3 participants, 6 of them recognized and appropriately responded to this sign by associating 940 and 60 in either their first strategy or second strategy. None of the six participants appropriately identified the associative property for addition by name. One participant correctly used order of operations but stated that the "associative property" was used. The same participant correctly used the associative property for addition as an alternate strategy but referred to it as the "commutative property." Four other MT3 participants correctly evaluated $940+(60+427)$ using only order of operations, and 6 others attempted, but incorrectly used, order of operations to evaluate $940+(60+427)$. These 10 MT3 participants failed to recognize the mathematical object behind the sign.

Associative property for multiplication. Q5 and Q10 were included to assess what participating PETs knew about the associative property for multiplication. For Q5 the mathematical object under consideration was the associative property for multiplication: "Without using a calculator find the value of $(72 \times 5) \times 2$ and explain how you got your answer." Three out of the 6 MT2 participants correctly recognized and responded to this sign by first computing $5 \times 2$ as either their first strategy or second strategy. The other three MT2 participants evaluated $(72 \times 5) \times 2$ using only order of operations; these participants failed to respond in a way that showed they recognized that the mathematical object under consideration was the associative property for multiplication. None of the MT2 participants used the words 
"associative property" or "associative property for multiplication." Five of the 16 MT3

participants correctly recognized and responded to the question above by first associating 5 and 2 as either their first strategy or second strategy. Only one of the MT3 participants used the words “associative property." Ten MT3 participants correctly evaluated $(72 \times 5) \times 2$ using only order of operations; these participants failed to respond in a way that showed they recognized that the mathematical object under consideration was the associative property for multiplication.

For Q10, MT2/MT3 participants were asked to find the value of $\left(120 \times \frac{1}{32}\right) \times 64$. Two of the 6 MT2 participants correctly recognized and responded to this sign by relying on the associative property for multiplication. However, neither of them used the words "associative property" or "associative property for multiplication." One of the participants gave a correct response using the associative property for multiplication but stated that the name (or names) of the property used was "commutative and additive inverse." One of the 16 MT3 participants correctly recognized and responded to the question by relying on the associative property for multiplication. However, that participant did not identify the associative property for multiplication by name. All other MT2 and MT3 participants failed to respond in a way which showed they recognized which mathematical object was under consideration.

Distributive property. Q4, Q7, Q8, and Q11 were included to assess what participating PETs knew about the distributive property (for multiplication over addition). For Q4, MT2/MT3 participants were asked to find the value of $x$ in $3 x=21 \times 3+4 \times 3$ using two different strategies. Of the 6 MT2 participants, only one addressed Q4 with two different strategies but neither of the strategies was the application of the distributive property. Three MT2 participants correctly addressed Q4 with one unique strategy, which was the use of order of operations to simplify the right-hand side of the equation and then dividing by 3 on both sides of the equal 
sign. Two MT2 participants incorrectly addressed Q4. In both their responses, the participants attempted to isolate the variable by first using order of operations to simplify the right-hand side of the equation.

\section{Table 8}

MT2/MT3 Responses to Distributive Property Questions

\begin{tabular}{|c|c|c|c|c|c|}
\hline Property & Q\# & & $\begin{array}{c}\text { No. of } \\
\text { Correct } \\
\text { Responses } \\
(\%)\end{array}$ & $\begin{array}{c}\text { No. Relying } \\
\text { on Property } \\
(\%)\end{array}$ & $\begin{array}{c}\text { No. } \\
\text { Naming } \\
\text { Property } \\
(\%)\end{array}$ \\
\hline \multirow{4}{*}{$\begin{array}{c}\text { Distributive } \\
\text { Property }\end{array}$} & Q4 & $3 x=21 \times 3+4 \times 3$ & $17(77 \%)$ & $0(0 \%)$ & $0(0 \%)$ \\
\hline & Q7 & $0.6 \times 250+250 \times 0.4$ & $11(50 \%)$ & $3(14 \%)$ & $1(5 \%)$ \\
\hline & Q8 & $\begin{array}{l}20 \times(10+5) \\
=(20 \times 10)+(20 \times y)\end{array}$ & $19(86 \%)$ & $6(27 \%)$ & $2(9 \%)$ \\
\hline & Q11 & $(1 / 5) \times 96+(1 / 5) \times 4$ & $5(23 \%)$ & $1(5 \%)$ & $0(0 \%)$ \\
\hline
\end{tabular}

Three out of 16 MT3 participants correctly addressed Q4 with two unique strategies. However, none of them used the distributive property as one of their strategies. The two strategies present in all 3 responses with two strategies were either (a) simplifying each side and then isolating the variable or (b) isolating the variable by first dividing each term in the equation by 3. Ten MT3 participants correctly responded to Q4 with one unique strategy—simplifying each side and then isolating the variable. Three participants incorrectly responded to Q4. These 3 participants attempted to address Q4 by simplifying each side and then isolating the variable. None of the MT2 or MT3 participants responded to Q4 in a way that the demonstrated that they understood the underlying mathematical object was the application of the distributive property.

For Q7, MT2/MT3 participants were asked to find the value of $0.6 \times 250+250 \times 0.4$ using two different strategies. Only one of the 6 MT2 participants correctly responded to Q7 
using two unique strategies; that participant used order of operations as one of the strategies and the distributive property as the alternate strategy. The participant did not identify the distributive property by name. One MT2 participant correctly responded to Q7 using one strategy—the order of operations. The other 4 participants incorrectly responded to Q7. The strategy of choice among these 4 participants in their attempt to respond to Q7 was the use of order of operations.

Only one MT3 participant correctly responded to Q7 with two unique strategies - the use of order of operations and the use of the distributive property without naming the property. Eight MT3 participants correctly responded to Q7 using one unique strategy. Seven of them relied on order of operations; one of them relied on the distributive property without identifying the property by name. All other responses by MT3 participants were incorrect, and the strategy employed in most of these responses was the use of the order of operations.

So, only one of the 4 MT2 participants and 2 of the 16 MT3 participants correctly recognized the mathematical object under consideration for Q7, but none of them used the words "distributive property." None of the other MT2 or MT3 participants responded in a manner which indicated they recognized the mathematical object under consideration; none of them used, or attempted to use, the distributive property. The preferred strategy for most participants was using order of operations.

For Q8, MT2/MT3 participants were asked to find the value of $y$ in $20 \times(10+5)=(20 \times$ $10)+(20 \times y)$. One MT2 participant appropriately responded to the question by using the distributive property; the participant also identified the property by name. Fourteen of the 16 MT3 participants correctly stated the value for $y$. Five MT3 participants appropriately responded to the question by using the concept of the distributive property, but only one of them actually used the words "distributive property." All other MT2 participants and most MT3 participants 
determined the value of $y$, or attempted to determine the value of $y$, by using order of operations and isolating the variable. These participants failed to recognize the underlying mathematical object behind the question.

For Q11, MT2/MT3 participants were asked to find the value of $\frac{1}{5} \times 96+\frac{1}{5} \times 4$. Only one MT2 participant correctly responded to the question by applying the distributive property but that student incorrectly used the words "commutative property." None of the MT3 participants appropriately responded to the sign; none of them used, or attempted to use, the distributive property. All other MT2 participants and most MT3 participants incorrectly evaluated, or did not complete evaluating, $\frac{1}{5} \times 96+\frac{1}{5} \times 4$; the strategy common in most of their responses was order of operations. Four MT2 participants correctly evaluated $\frac{1}{5} \times 96+\frac{1}{5} \times 4$ using order of operations, but these participants failed to recognize the underlying mathematical object behind the question.

\section{Other Questions on the Paper-and-Pencil Instruments}

Two questions, Q6 and Q13, were included to assess whether participating PETs would inappropriately apply a number property to an operation in which the property does not hold. For Q6 on all assessments - including both the pre-teaching and post-teaching assessments issued to MT1 participants and the assessments issued to MT2 and MT3 participants_-participants were asked to determine the value of $x$ given $12-(8-4)=(12-x)-4$. This question was included to see whether participating PETs would inappropriately apply the associative property to the subtraction operation.

Sixteen MT1 participants completed the pre-teaching instrument, and 12 of them incorrectly answered Q6. Most MT1 participants who incorrectly responded to Q6 on the preteaching instrument attempted to simplify each side of the equal sign so to isolate the variable. 
However, there were 3 participants who incorrectly concluded that $x=8$. Although not explicitly stated by of the 3 participants, it is possible that these participants inappropriately applied the associative property to the subtraction operation. Sixteen MT1 participants completed the postteaching instrument. There were 14 incorrect responses to Q6 on the post-teaching instrument among MT1 participants, and 10 of them incorrectly concluded that $x=8$. It is possible that these 10 participants incorrectly concluded that $x=8$ because they inappropriately applied the associative property to the subtraction operation. However, only a handful of them explicitly stated so. Three of them explicitly stated they were using the "associative property" (or "associative property for subtraction"), and one other stated that the property used to conclude that $x=8$ was the "commutative property."

MT2 and MT3 only completed one assessment. Six MT2 participants completed the assessment. Three of the 6 MT2 participants correctly responded to Q6. Three others incorrectly answered this question or did not complete the question; one of them wrote that " $x=8$ ", and another remarked that she was unsure whether correct answer was $x=8$ or $x=0$. If an individual incorrectly assumes that the associative property holds for subtraction, then the individual would be likely to conclude incorrectly that for the equation $12-(8-4)=(12-x)-4, x=8$. Sixteen MT3 participants completed the assessment, and 9 of them correctly answered Q6. There were 7 incorrect responses to Q6 among the MT3 participants. Out of the 7 incorrect responses, 5 of them incorrectly concluded that $x=8$. Although not explicitly stated by of the 5 participants, it is possible that these participants inappropriately applied the associative property to the subtraction operation.

For Q13 on the MT1 pre-teaching instrument, participants were asked to find the value of $y$ given $(10-y)-(10-5)=0$. Out of 16 participating MT1 PETs, 10 of them correctly 
responded to Q13 on the pre-teaching instrument. Six of these 10 MT1 PETs solved for $y$ by first simplifying each side of the equation and then isolating the variable; four of them recognized that $10-y$ must equal $10-5$ for the difference to equal 0 . One of the MT1 participants recognized that $y=5$ if $10-y$ equals $10-5$, but then erroneously stated that she used the "substitution property."

For Q13 on the MT1 post-teaching instrument, participants were asked to find the value of $y$ given $(15-y)-(15-5)=0$. Of the 16 participating MT1 PETs, 14 of them correctly responded to Q13 on the post-teaching assessment. Two of these 14 MT1 PETs solved for $y$ by first simplifying each side of the equation and then isolating the variable; five of them recognized that $10-y$ must equal $10-5$ for the difference to equal 0 . One participant correctly stated that $y=5$ but erroneously wrote, " $y$ must equal 5 in order to abide by the associative property." Another participant wrote, " $y=5$ due to the associative prop. \& additive inverse property." The associative property does not hold for subtraction, and it is not evident that the associative property for addition (or multiplication) could be applied to readily determine the value for $y$ in $\mathrm{Q} 13$.

For Q13, MT2/MT3 participants were also expected to find the value of $y$ given $(10-y)$ $-(10-5)=0$. Six MT2 participants completed the assessment, and all 6 correctly answered Q13. None of them mentioned any properties in their responses. Sixteen MT3 participants completed the assessment, and 13 of them correctly answered Q13. Eleven of them recognized $y$ $=5$ because $10-y$ must equal $10-5$. None of the MT3 participants mentioned relying on any properties in their responses. Two other questions, Q14 and Q15, were included to assess whether participating PETs could explain using number properties, the steps taken by some hypothetical students in evaluating a numerical expression. For Q14, participants were asked to 
identify the appropriate expression for each step corresponding the property used to move from one step to the next. For Q15, participants were asked to identify the appropriate property used to move from one step to the next. Refer to Figure 5 for the detailed versions of Q14 and Q15. Parallel versions of Q14 and Q15 were written for the post-teaching instrument issued to MT1 participating PETs.

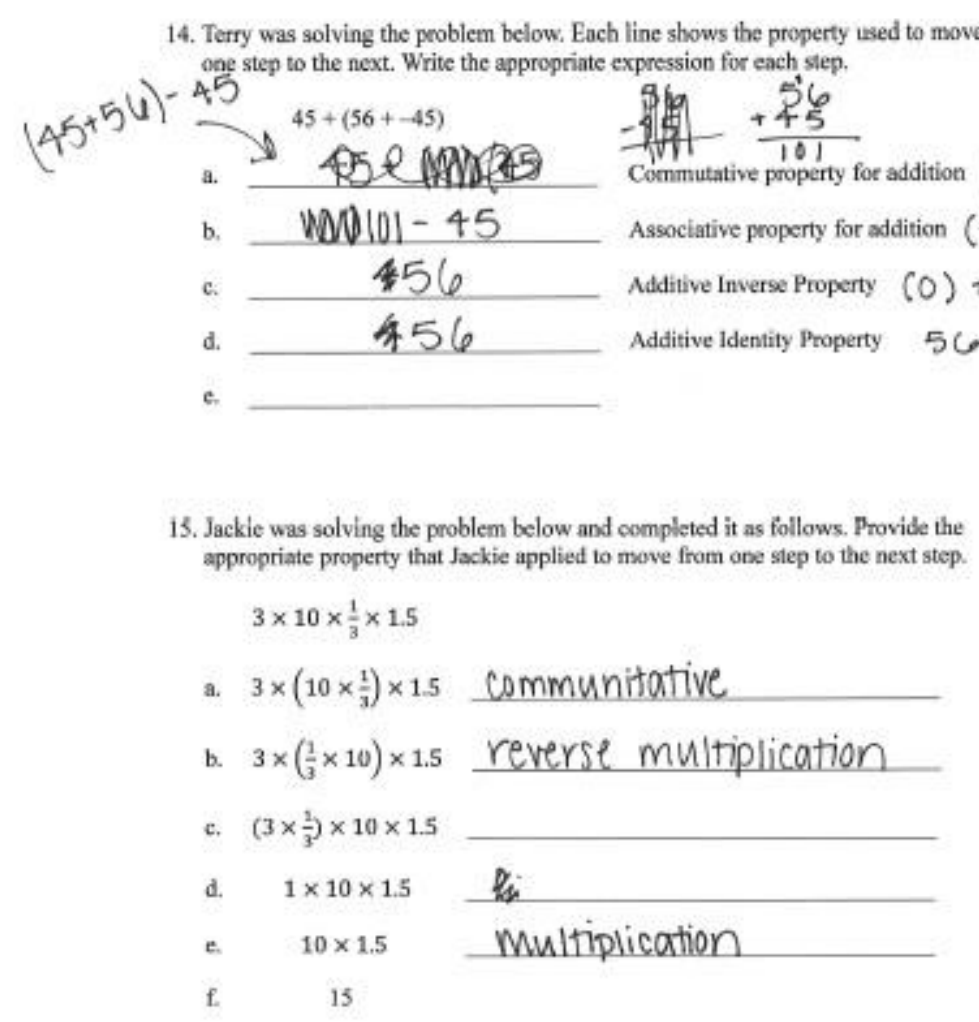

Figure 5. A sample response to Q14 and Q15 from one of the MT3 participants.

A sample response to Q14 and Q15 from one of the MT3 participants is shown in Figure 5. Of the 16 MT1 participants who completed the pre-teaching instrument, none of them responded adequately to Q14 or Q15. Of the $16 \mathrm{MT} 1$ participants who completed the postteaching instrument, there were 2 participants who responded adequately to Q14 and Q15. None of the 6 MT2 participants who completed the assessment adequately responded to Q14 or Q15. Two MT3 participants responded adequately to Q14. 


\section{Interview Data Findings}

\section{MT1 PETs (Ally and Betty)}

Two MT1 PETs, Ally and Betty (pseudonyms), agreed to participate in audio-taped interviews. Based on pre-teaching assessment data, Ally was categorized as a high performing student, and Betty was categorized as an average performing student. Ally's pre-teaching assessment score was 2 standard deviations above the class mean, and Betty's score was within 2 standard deviations of the class mean. Ally and Betty participated in both the pre-teaching and post-teaching interviews (see Appendix G for interview data). Both the pre-teaching and postteaching interviews began with me, the interviewer, asking the participants to describe the distributive property. I started each interview by making the following statement:

I am going to say two words to you and, as soon as I say them, I want you to say something, or draw something, or do something — do the first thing that comes into your head after I say the words. Here are the words ... "distributive property." Here are the words again: "distributive property." What comes into your mind?"

Knowledge of the distributive property. In both the pre-teaching and post-teaching interviews, both Ally and Betty gave appropriate examples of the distributive property. Even though they both generated appropriate examples of the distributive property, they did not consistently apply the property in relevant situations. When they were asked to calculate the value of $315 \times 0.7+315 \times 0.3$ (during the pre-teaching interview) and the value of $7 \times 97+7 \times$ 3 (during the post-teaching interview), neither of them willingly employed the distributive property. They did not recognize that the rules for order of operations were superseded by the distributive property when calculating the values of $315 \times 0.7+315 \times 0.3$ and $7 \times 97+7 \times 3$, even after teaching had taken place. 
Ally and Betty were asked to compute the value of $92 \times 5$ (during the pre-teaching interview) and the value of $84 \times 5$ (during the post-teaching interview) and to describe whether a number property was applied in their multiplication strategy. During the pre-teaching interview, Ally guessed that property used in her algorithm was the distributive property. During the postteaching interview, she recognized that $84 \times 5=80 \times 5+4 \times 5$ but could not name the property she was using. During the pre-teaching interview, Betty could not justify why her procedure for multiplying was valid. During the post-teaching interview, she was more successful at articulating why her strategy works. Betty recognized that $84 \times 5=80 \times 5+4 \times 5=400+20=$ 420. However, when asked if any property was used, she replied, "associative property."

During the post-teaching interview, Ally and Betty were asked to determine the value of $x$ that would make the following equation true: $5.25 x=70 \times 5.25+30 \times 5.25$. In order to find the value $x$, Ally relied on the concept of factoring but did not connect it to the distributive property. Betty was not able to determine successfully the value of $x$. Her strategy for finding the value of $x$ was incorrect and inappropriate for this prompt.

The interview data suggests that both Ally's and Betty's concept image of the distributive property did not change in a qualitatively significant way between the pre- and post-teaching stages. They did not develop a well-formed concept image of the distributive property. Ally, for example, factored when solving for $x$ in $5.25 x=70 \times 5.25+30 \times 5.25$ but did not use the words "distributive property." Betty recognized that $84 \times 5=80 \times 5+4 \times 5$ but incorrectly used the words "associative property." Ally and Betty both provided appropriate examples of the distributive property but did not recognize arithmetic patterns, such as $0.8 \times 315+315 \times 0.2$, for which using the distributive property would be appropriate. This illustrates "the fact that someone's concept image with respect to some topic is not made up solely of separate 
components (verbal, skills, imagery, episodes, [and] attitudes). Cognitive links, or relationships between the components, are also important in defining the concept image" (Kanbir et al., 2018, p. 164).

Knowledge of the commutative property for addition. During the pre-teaching interviews, Ally and Betty were asked to solve for $y$ in $13+y=14+13$. Ally's strategy of choice during the pre-teaching interview was to determine the value of $y$ by isolating the variable. When asked about an alternative way of determining the value $y$, Ally referred to idea of "canceling" the common term on both sides of the equal sign. When pressed to state whether she used any properties, she replied, "Yes ... subtraction." Based on her responses, Ally did not demonstrate awareness of the how the commutative property for addition could be correctly applied. She did not directly employ or mention the commutative property for addition when responding to the task of solving for $y$ in $13+y=14+13$. When calculating the value of $y$ in in $13+y=14+13$ during the pre-teaching interview, Betty argued that what is done one side of the equal sign must be done to the other side. She contended that if we start with 13 equals 13 , then adding 14 to 13 on one side of the equal sign means that 14 must also be added to 13 on the other side of the equal sign. When asked about the property that applied, she responded, "The commutative property, but I don't know if that's right." Although Betty demonstrated awareness of the commutative property for addition, she did not directly employ the property when solving for $y$ in $13+y=14+13$.

During the post-teaching interviews, Ally and Betty were asked to solve for $y$ in $14+5=$ $5+y$. Ally's strategy of choice for determining the value of $y$ during the post-teaching interview was the same as the one used during the pre-teaching interview. She determined the value of y by isolating the variable. In both the pre-teaching and post-teaching interviews, Ally did not exhibit 
a willingness to use the commutative property for addition. The evidence does not suggest any change in Ally's concept image of the commutative property for addition. During the postteaching interview, Betty correctly stated the value of $y$ but stated that the property used was "the associative property." When asked to elaborate, she described a strategy in which she determined the value for $y$ by subtracting 5 from the sum of the left-hand side of the equal sign. The evidence does not indicate improvement in Betty's concept image of the commutative property for addition between the pre-teaching and post-teaching stages.

Knowledge of the commutative property for multiplication. During the pre-teaching interviews, Ally and Betty were asked to solve for $y$ in $5 y=4 \times 5$. Ally's strategy of choice during the pre-teaching interview was finding the value of $y$ by dividing both sides of the equal sign by 5 . She used that approach to obtain $y=4$. When asked whether there was another way she could determine the value $y$, she responded by cancelling the number 5 on both sides of the equal sign. When pressed on whether she used any properties, she replied, "division." Ally did not demonstrate an awareness of how the commutative property for multiplication might be involved. She did not directly employ or mention the commutative property for multiplication when responding to the task of solving for $y$ in $5 y=4 \times 5$. Betty's strategy of choice during the pre-teaching interview was determining the value for $y$ by dividing 5 into the product of the numbers on the right-hand side of the equal sign. She correctly identified $y=4$ but did not use or mention the commutative property for multiplication.

During the post-teaching interviews, Ally and Betty were asked to solve for $y$ in $4 \times 112$ $=112 y$. Ally did not correctly determine the value of $y$ which would make the equation, $4 \times 112$ $=112 y$, true. Betty divided both sides of the equal sign by 112 to determine the value for $y$. During both the pre-teaching and post-teaching interviews, neither Ally nor Betty referred to the 
commutative property for multiplication when finding the value for $y$. Both Ally and Betty did not respond to the tasks in ways which demonstrated an awareness of the underlying mathematical object. They did not demonstrate awareness of how and when the commutative property for multiplication can be applied. The evidence does not suggest a change between the pre- and post-teaching stages in either Ally's or Betty's concept image of the commutative property for multiplication.

Knowledge of the associative property for addition. One of the questions on both the pre-teaching and post-teaching instrument required participants to describe the associative property for addition. Out of 16 participating MT1 PETs, only 2 of them correctly described the associative property for addition on the pre-teaching instrument. On the post-teaching instrument, 7 of the 16 participating MT1 PETs who completed this assessment correctly described the associative property for addition. Neither Ally nor Betty correctly described the associative property for addition on the pre-teaching instrument. During the pre-teaching interviews, Ally and Betty were asked to evaluate $482+(18+300)$. Ally initially described evaluating the expression by adding 482 to 318 . When she was asked to describe an alternative strategy, she described adding 482 to 18 and then adding that sum to 300 , which she described as “the property for addition." Betty also calculated $482+(18+300)$ using order of operations. She recalled: "So, this takes me back. Uh, there was a principle I was taught called PEMDAS: Please Excuse My Dear Aunt Sally. It's pretty much the order of operations ... So, with $18+300$ in the parentheses, that is $318 \ldots$ So, you have $482+318 \ldots$ So, your answer would be $800 . "$ When asked to describe an alternative strategy, Betty replied, “I don’t think so. I don't know any. If there are any, I don't know any." 
At the pre-teaching stage, neither Ally nor Betty accurately expressed in writing the meaning of the associative property for addition. Neither correctly responded, either receptively nor expressively, to questions asked during the pre-teaching interview for which the underlying mathematical object was the associative property for addition. On the post-teaching instrument, Ally appropriately described the associative property for addition, but Betty's description was erroneous. During the post-teaching interviews, Ally and Betty were asked to evaluate $940+(60$ +403 ), and although they both evaluated the expression by relying on the associative property for addition as a first strategy, they both incorrectly identified the property used as "the commutative property."

Between pre-teaching and post-teaching stages, Ally's concept image of the associative property for addition positively changed with respective to verbal knowledge, intellectual skills, and imagery. At the post-teaching stage, Ally accurately described, both receptively and expressively, the associative property for addition. She responded to the task of evaluating $482+$ $(18+300)$ in a manner which demonstrated she understood that applying the associative property for addition superseded relying on the rules for order of operations. However, after evaluating $482+(18+300)$ using the associative property for addition, she referred to the property used as the "commutative property." Even though Ally's concept image of the associative property for addition had changed, she had not yet developed a well-formed concept image of the property.

Between the pre- and post-teaching stages, Betty's concept image of the associative property for addition changed positively with respective to intellectual skills, and imagery. With respect to verbal knowledge at the post-teaching stage, Betty did not accurately describe the associative property for addition. However, she did respond to the task of evaluating $482+(18+$ 
300) in a manner which demonstrated that she was aware that thinking of $482+(18+300)$ as $(482+18)+300$ superseded relying on the rules for order of operations. However, she incorrectly referred to the property as the "commutative property." Even though Betty's concept image for the associative property for addition changed, she had not yet developed a well-formed concept image of the property.

Knowledge of the associative property for multiplication. During the pre-teaching interviews, Ally and Betty were asked to evaluate $4 \times(1 / 4 \times 128)$. They both relied on order of operations as a first strategy. However, when asked to describe an alternative strategy, only Ally recognized that the 4 and $1 / 4$ in $4 \times(1 / 4 \times 128)$ could be associated, but she did not identify the associative property for multiplication by name. When asked to identify the property that allowed her to associate the 4 and $1 / 4$, she responded, "inverse operations." This response was partially appropriate because 4 and $1 / 4$ are multiplicative inverses.

During the post-teaching interviews, Ally and Betty were asked to evaluate $(72 \times 5) \times 2$ and $64 \times\left(\frac{1}{32} \times 120\right)$. When calculating the value of $(72 \times 5) \times 2$, both Ally and Betty relied on the associative property for multiplication as a first strategy. However, as with the associative property for addition, both misidentified the associative property for multiplication as the commutative property. When calculating the value of $64 \times\left(\frac{1}{32} \times 120\right)$, only Ally associated 64 and $\frac{1}{32}$. Even though Ally recognized that 64 and $\frac{1}{32}$ could be associated, she again misidentified the property she had used as the commutative property of multiplication. Betty did not successfully calculate the value of $64 \times\left(\frac{1}{32} \times 120\right)$. Even though Betty was unsuccessful in evaluating $64 \times\left(\frac{1}{32} \times 120\right)$, she recognized that 64 and $\frac{1}{32}$ could be associated. However, she was not sure about why she might be allowed to link the 64 and the $\frac{1}{32}$. She was not confident on how 
to deal with the fraction $\frac{1}{32}$. When asked about the property that would allow her to associate 64 and $\frac{1}{32}$, she misidentified it as the commutative property.

Both Ally's and Betty's concept image of the associative property for multiplication changed between the pre-teaching and post-teaching stages. At the post-teaching stage, they both demonstrated improved skills and imageries for the associative property for multiplication.

During the post-teaching interviews, both Ally and Betty demonstrated a willingness to directly apply the associative property for multiplication to calculate the values of $(72 \times 5) \times 2$ and $64 \times$ $\left(\frac{1}{32} \times 120\right)$. They demonstrated that they understood that associating 5 and 2 in $(72 \times 5) \times 2$ and associating 64 and $\frac{1}{32}$ in $64 \times\left(\frac{1}{32} \times 120\right)$ superseded using the rules for order of operations. However, they both confused the names of the associative properties with those of the commutative properties.

Knowledge of inverse and identity properties. In both the pre- and post-teaching interviews, Ally and Betty were asked to describe the meanings of the following terms: additive identity, additive inverse, multiplicative identity, and multiplicative inverse. They did not demonstrate strong understanding of the meanings of these terms in either pre- or post-teaching interviews. At both the pre-teaching and post-teaching stages, Ally could not provide appropriate descriptions of any of the identity or inverse properties when prompted to do so. She could not recall from memory anything she had ever learned about identity or inverse properties. She demonstrated some knowledge of the concept of an inverse but did not exhibit any well-formed knowledge or imagery of the additive inverse property or the multiplicative inverse property. At the pre-teaching stage, Betty stated she had not previously seen, or been taught the meaning of the identity or inverse properties. By the post-teaching stage, she was able to describe the 
meanings of the terms "additive inverse" and "multiplicative identity" but could not describe the meaning of "additive identity" and gave an incorrect description of "multiplicative inverse."

\section{MT2/MT3 PETs (Casey and Daisy)}

One MT2 participant, Casey (pseudonym), and one MT3 participant, Daisy (pseudonym), agreed to participate in audio-taped interviews (see Appendix G for interview data). Both Casey and Daisy were average performing students with their scores being within 2 standard deviations of their respective class means. Casey and Daisy only completed one interview each because there was no teaching intervention in the MT2 and MT3 courses.

Knowledge of the distributive property. Both Casey and Daisy were asked to describe the distributive property during their interviews, but only Casey provided an appropriate description. Even though Casey provided an appropriate description of the distributive property, she stated that she was not sure that she was right. Even though Casey had previously provided an appropriate description of the distributive property, she did not recognize that the property could be used to evaluate $315 \times 0.7+315 \times 0.3$ readily. In determining the value of $x$ in $5.25 x=$ $70 \times 5.25+30 \times 5.25$, Casey's first strategy of choice was dividing all terms in the equation by 5.25 and then simplifying the right-hand side of the equation by finding the sum of 70 and 30 . When asked to describe any other suitable strategy for finding the value of $x$, Casey factored 5.25 on the right-hand side yielding $5.25 x=5.25 \times(70+30)$. When asked if she used any properties, she responded, "equality ... is that the property?" Casey did not connect factoring to the distributive property.

Daisy did not provide an appropriate example or description of the distributive property. She relied on order of operations to evaluate $315 \times 0.7+315 \times 0.3$ or solve for $x$ in $5.25 x=$ $70 \times 5.25+30 \times 5.25$. Thus, Daisy demonstrated no evidence that she knew what the 
distributive property was or how it could be applied. Casey demonstrated some knowledge of the distributive property but did not possess a well-formed concept image. Both Casey and Daisy did not recognize that the rules for order of operations were superseded by the distributive property; they did not recognize the arithmetic patterns for which the distributive property was appropriate.

Knowledge of the commutative property for addition. Casey and Daisy were asked to evaluate $13+y=14+13$ during their interviews. Both Casey and Daisy correctly stated that the value for $y$ was 14, but they did not offer any explanation. When Daisy was asked to explain how she obtained $y=14$, she responded, "Because you will subtract 13 from both sides. You're left with $y=14$." However, when she was asked to describe another way of explaining why $y=14$, she could not provide one. Daisy did not demonstrate knowledge, or awareness of how the commutative property for addition could be recognized or applied. When Casey was asked to explain how she arrived at $y=14$, she responded, "I would subtract 13 from the sides, and this would cancel out, so $y$ would be 14 ." When asked for an alternative explanation for why $y=14$, Casey referred to the "reflexive property." When pressed about what that property stated, she was unsure whether it described $a=a$, or $a+b=b+a$. She did, however, clarify that she knew $y=14$ because $a+b=b+a$. Casey did not initially rely on the commutative property for addition but later acknowledged the role of that property in explaining why $y=14$.

Knowledge of the commutative property for multiplication. Casey and Daisy were not presented with a "commutative property for multiplication" task during the interview. Instead, their responses on the paper-and-pencil assessments were examined to ascertain what they knew and understood about the commutative property for multiplication. On the paper-and-pencil assessment, MT2/MT3 were asked to describe the commutative property for multiplication. Neither Casey nor Daisy correctly described, in writing, the commutative property for 
multiplication. Casey described the distributive property instead, and Daisy wrote, "I don’t know." The following question on the paper-and-pencil instrument was framed with a certain mathematical object in mind-the commutative property for multiplication: "If $13 \times y=9 \times 13$, what must $y$ equal? Which property allows you to quickly determine the value of $y$ ?" Neither Casey nor Daisy responded in a way that demonstrated that they recognized the mathematical object under consideration. Casey gave the correct answer for $y$, but wrote, questioning, “transitive?” Daisy gave the correct answer for $y$, but wrote, "I don't know what property allows me to quickly determine the value of $y . "$

Knowledge of the associative property for addition. MT2/MT3 PETs only completed one pencil-and-paper instrument. Only 1 of the 6 participating MT2 PETs correctly described the associative property for addition, and none of the participating MT3 PETs correctly described the property. Neither Casey nor Daisy correctly described, in writing, the associative property for addition. During the audio-taped interviews, both Casey and Daisy relied on order of operations as their first strategy for evaluating $482+(18+300)$. Casey's first attempt to evaluate $482+(18$ $+300)$ led her to conclude, incorrectly, that the value of $482+(18+300)$ is 600 . When asked to describe an alternate strategy, she drew a number line in which she started with 482, added 300, and then added 18. She realized that her initial answer was incorrect and corrected it to 800 . When Casey was asked whether she used any properties, she replied with a question, "the addition property?" Daisy evaluated $482+(18+300)$ by first computing $18+300$ and then adding this sum to 482. When she was asked to explain, she responded, "You do the 'Please Excuse My Dear Aunty Sally." When asked to describe alternative strategy, Daisy responded, “Add 300 to 482 to get 782, and then add the 18." When asked whether she used any properties, 
she replied, "I don't know." Neither Casey nor Daisy had a well-formed concept image of the associative property for addition.

Knowledge of the associative property for multiplication. Casey and Daisy were asked to evaluate $4 \times(1 / 4 \times 128)$ during their interviews. They both relied on order of operations as a first strategy for calculating the value of $4 \times(1 / 4 \times 128)$. Casey initially emphasized that she needed to first calculate what was in the parenthesis. When asked to describe an alternate strategy, she responded, "If I could just get the thought out of my head that I have to acknowledge the parentheses. Um, I would know that $4 \times 1 / 4$ is 1 , and then that would be $1 \times$ 128." When asked to about the property employed, she responded with a question:

“Multiplication?" Daisy evaluated $4 \times(1 / 4 \times 128)$ by first computing $1 / 4 \times 128$. She changed $1 / 4$ into a decimal and computed $0.25 \times 128$. Daisy stated that she changed the fraction to its decimal equivalent because she was more comfortable with decimals than fractions. When asked to use an alternate strategy to evaluate $4 \times(1 / 4 \times 128)$, Daisy did not provide one. Both Casey and Daisy did not respond in a manner which demonstrated well-formed concept images of the associative property for multiplication. Neither showed that they understood that the rules for order of operations were superseded by the associative property for multiplication.

Knowledge of inverse and identity properties. Casey and Daisy were also asked to describe the meanings of the following terms during their interviews: additive identity, additive inverse, multiplicative identity, and multiplicative inverse. They demonstrated practically no knowledge of the meanings of any of those terms. They could not recall learning anything about any of the identity or inverse properties. They guessed at what the terminology meant but neither provided appropriate descriptions. 


\section{Quantitative Results}

There were 15 questions on the paper-and-pencil instruments. Nine questions, presented previously in Table 2 in abbreviated format, specifically assessed whether participating PETs could recognize and use number properties appropriately to simplify expressions or solve equations, and to assess whether they could identify these properties by name. Hereafter, these nine questions will be referred to as the "nine-property questions." Participating MT1 PETs completed and responded to a pre-teaching and post-teaching assessment instrument that were administered before and after participating in instruction emphasizing number properties. Participating MT2/MT3 PETs did not participate in instruction emphasizing number properties and, therefore, only completed one assessment instrument. Because of the lack of clear distinction between participating MT2 and MT3 PETs, their data was combined into one group - MT2/MT3 PETs—and studied as one.

Each assessment instrument completed by the participating PETs was evaluated and assigned four different scores: an overall score for the entire assessment, a score on the nineproperty questions, a score on the usage of properties as evidenced on the nine-property questions (hereafter, referred to as "usage of properties score"), and a score on identifying properties by name as evidenced on the nine-property questions (hereafter, referred to as "naming properties score"). The overall score was determined by finding the sum of scores on the 15 questions based on the correctness of the responses to each question on the paper-andpencil instruments. The maximum possible overall score was 15 . For the nine-property questions, each question was scored on three levels: correctness, application of the appropriate mathematical property, and identification of the name of the property. This resulted in three categories of scores: the nine-property questions (NPQ) score, the usage of properties (UOP) 
score, and the naming properties (NP) score. The maximum possible score for these three types of scores was 9 .

This study was carried out with non-random samples. There was no attempt to control the allocation of students to the three classes - the individual participants were not randomly allocated to the three classes from any well-defined population. Repeated-measures $t$-tests and independent samples $t$-tests were used as statistical tools whereby comparisons were made between the groups in the study. These statistical tools were only used in this fashion and were not used for the purpose of making generalizations about population parameters. It was recognized that because this study did not make use of random samples, it would not be appropriate use statistical tests to draw general conclusions about population parameters.

\section{Pre-Teaching vs. Post-Teaching Performance of MT1 PETs}

Repeated-measures $t$-tests were conducted to test for differences in the mean performances of MT1 PETs on the pre- and post-teaching instruments. It should be noted that even though there were 17 participating MT1 PETs, one of the MT1 PETs only completed the pre-teaching assessment instrument and another only completed the post-teaching assessment instrument because of absences when then the instruments were administered. So, only 15 participating MT1 PETs completed both the pre-teaching and post-teaching instruments, and the scores of these 15 participating PETs were the only ones included when conducting the repeatedmeasures $t$-test. The repeated-measures $t$-test was conducted for each of the four types of scores in order to determine whether there was a statistically significant difference between MT1 PETs' pre- and post-teaching mean scores. The repeated-measures $t$-tests used $\alpha=0.05$ as the criterion for significance. 
The first repeated-measures $t$-test was conducted to test whether there was a statistically significant difference in the overall score of MT1 PETs on the pre- and post-teaching instruments. The following null hypothesis was considered: the pre- and post-teaching overall scores for each group were not statistically significantly different. If the null and alternative hypotheses are denoted by $\mathrm{H}_{0}$ and $\mathrm{H}_{1}$, then:

$\mathrm{H}_{0}$ : The difference between the mean of the pre-teaching overall scores and the mean of the post-teaching overall scores would be equal to zero.

$\mathrm{H}_{1}$ : The difference between the mean of the pre-teaching overall scores and the mean of the post-teaching overall scores would not be equal to zero.

This test was statistically significant, $t(14)=2.69, n=15, p=0.02,95 \%$ C. I. [0.30, 2.64]. The null hypothesis can be rejected. The mean overall score of MT1 PETs on the post-teaching instrument $(M=7.29, S D=2.12)$ was statistically significantly higher than the mean overall score on the pre-teaching instrument $(M=5.83, S D=2.34)$.

The second repeated-measures $t$-test was conducted to test whether the mean of MT1 PETs' pre-teaching scores on the nine property questions (NPQ) was statistically significantly different from the mean of the post-teaching scores. The following null hypothesis was considered for this test: the means of the pre-teaching and post-teaching NPQ scores were not statistically significantly different. The null and alternative hypotheses were:

$\mathrm{H}_{0}$ : The difference between the means of the pre- and post-teaching NPQ scores would be equal to zero.

$\mathrm{H}_{1}$ : The difference between the means of the pre-t and post-teaching NPQ scores not be equal to zero. 
This test did not yield a statistically significant, result: $t(14)=1.10, n=15, p=0.288,95 \%$ C. I. $[0.38,1.18]$. The null hypothesis cannot be rejected. The mean NPQ score of MT1 PETs on the post-teaching instrument $(M=5.10, S D=1.48)$ was not statistically significantly higher than the mean NPQ score on the pre-teaching assessment $(M=4.70, S D=1.66)$. On average, the score for the nine property questions on the post-teaching assessment completed by MT1 PETs was 0.40 points higher than the score for the nine property questions on the pre-teaching assessment.

The third repeated-measures $t$-test was conducted to test whether the mean of MT1 PETs' pre-teaching UOP scores was statistically significantly different from the mean of the postteaching UOP scores. The following null hypothesis was considered for this test: the means of the pre-teaching and post-teaching UOP scores were not statistically significantly different. The null and alternative hypotheses were:

$\mathrm{H}_{0}$ : The difference between the means of the pre- and post-teaching UOP scores would be equal to zero.

$\mathrm{H}_{1}$ : The difference between the means of the pre-t and post-teaching UOP scores not be equal to zero.

This test yielded a statistically significant, result: $t(14)=4.97, n=15, p<0.01,95 \%$ C. I. [1.14, 2.86]. The null hypothesis can be rejected. The mean UOP score of MT1 PETs on the postteaching instrument $(M=3.00, S D=1.56)$ was statistically significantly higher than the mean UOP score on the pre-teaching instrument $(M=1.00, S D=1.07)$. On average, the UOP scores on the post-teaching instrument completed by MT1 PETs were 2.00 points higher than the UOP scores on the pre-teaching instrument.

The fourth repeated-measures $t$-test was conducted to test whether the mean of MT1 PETs' pre-teaching NP scores was statistically significantly different from the mean of the post- 
teaching NP scores. The following null hypothesis was considered for this test: the means of the pre-teaching and post-teaching NP scores were not statistically significantly different. The null and alternative hypotheses were:

$\mathrm{H}_{0}$ : The difference between the means of the pre- and post-teaching NP scores would be equal to zero.

$\mathrm{H}_{1}$ : The difference between the means of the pre-t and post-teaching VP scores not be equal to zero.

This test was statistically significant, $t(14)=3.00, n=15, p=0.01,95 \%$ C. I. [0.38, 2.29]. The null hypothesis can be rejected. The mean NP score of MT1 PETs on the post-teaching instrument $(M=1.53, S D=1.64)$ was statistically significantly higher than the mean NP score on the pre-teaching instrument $(M=0.20, S D=0.56)$. On average, the NP scores on the postteaching instrument completed by MT1 PETs were 1.33 points higher than the NP scores on the pre-teaching instrument.

Cohen's $\boldsymbol{d}$ effect sizes and Performance Improvement Ratios. The repeated-measures $t$-tests were used in this study to assess whether the pre-teaching means and post-teachings means on assessments completed by participating MT1 PETs were statistically significantly different. The repeated-measures $t$-tests were used only in this fashion and not to make inferences about population parameters. The tests revealed that the pre-teaching means and postteachings means for the overall score, the usage of properties score, and the naming properties score were statistically significantly different. Statistical significance, by itself, is not meaningful without consideration of the size of effect (Coe, 2002; Fan, 2001; Sullivan \& Feinn, 2012). Fan (2001) wrote that statistical significance and effect size could be thought of as two sides of the same coin which complement each other. Because statistical significance and effect size are 
complementary, Fan suggested that these measures should be used in tandem rather than as a substitute for the other.

Cohen's $d$ effect sizes (Cohen, 1988) were calculated to measure the size of the effect of teaching in MT1. Cohen's $d$ effect size was calculated by subtracting the mean of the postteaching scores from the mean of the pre-teaching scores and dividing the difference by the pooled standard deviation:

$$
d=\frac{\text { Mean }_{\text {post }}-\text { Mean }_{\text {pre }}}{\sqrt{\left(\frac{S D_{\text {post }}{ }^{2}}{2}+\frac{S D_{\text {pre }}{ }^{2}}{2}\right)}}
$$

Cohen's $d$ "has become the measure of choice for effect size" (Fan, 2001, p. 277). Cohen (1988) presented the following conventions for interpreting effect size: an effect size of $d=0.2$ would be considered as "small," an effect size of $d=0.5$ would be considered as "medium," and an effect size of $d=0.8$ or higher would be considered as "large." According to Cohen's conventions, an effect size of $d=0$ would imply that the pre-teaching and post-teaching means have $0 \%$ non-overlap - the pre-teaching mean is the same as the post-teaching mean; an effect size of $d=0.2$ would imply that the pre-teaching and post-teaching means have a non-overlap of about $14.7 \%$; an effect size of $d=0.5$ would imply that the pre-teaching and post-teaching means have a non-overlap of about 33.0\%; and an effect size of $d=0.8$ would imply that the preteaching and post-teaching means have a non-overlap of about $47.4 \%$.

In addition to calculating Cohen's $d$ effect sizes, performance improvement ratios were also calculated as an additional measure for quantifying the effect of teaching in MT1. Duarte (2010) used performance improvement ratios as one of the ways for quantifying the effect between pre-teaching and post-teaching stages. Duarte (2010) described the performance improvement ratio (PIR) as the "ratio of the difference between post-teaching and pre-teaching 
mean scores to the difference of maximum possible test score and pre-teaching mean score" (p. 216). For example, the means for the pre-teaching and post-teaching overall scores were 5.83 and 7.29, respectively, out of a maximum possible score of 15 . For this example, the PIR would be calculated as $\frac{7.29-5.83}{15-5.83}=0.16$. This PIR implies that the overall score mean gain was $16 \%$ of the maximum possible gain achievable. In Table 9, Cohen's $d$ effect sizes and PIRs are summarized.

Table 9

Cohen's d effect sizes and PIRs for MT1 pre-and post-teaching scores

\begin{tabular}{|c|c|c|c|c|c|c|c|}
\hline Score & & $n$ & $\begin{array}{l}\text { Max. } \\
\text { Score }\end{array}$ & Mean & $S D$ & $\begin{array}{l}\text { Cohen's } d \\
\text { Effect Size }\end{array}$ & PIR \\
\hline \multirow{2}{*}{ Overall } & Pre-T & 15 & 15 & 5.83 & 2.34 & \multirow{2}{*}{0.65} & \multirow{2}{*}{0.16} \\
\hline & Post-T & 15 & 15 & 7.29 & 2.12 & & \\
\hline \multirow{2}{*}{ NPQ } & Pre-T & 15 & 9 & 4.70 & 1.66 & \multirow{2}{*}{0.25} & \multirow{2}{*}{0.09} \\
\hline & Post-T & 15 & 9 & 5.10 & 1.48 & & \\
\hline \multirow{2}{*}{ UOP } & Pre-T & 15 & 9 & 1.00 & 1.07 & \multirow{2}{*}{1.50} & \multirow{2}{*}{0.25} \\
\hline & Post-T & 15 & 9 & 3.00 & 1.56 & & \\
\hline \multirow{2}{*}{ NP } & Pre-T & 15 & 9 & 0.20 & 0.56 & \multirow{2}{*}{1.09} & \multirow{2}{*}{0.15} \\
\hline & Post-T & 15 & 9 & 1.53 & 1.64 & & \\
\hline
\end{tabular}

\section{MT1 vs. MT2/MT3 Performance}

In addition to testing whether there was a statistically significant difference between the pre- and post-teaching performances of MT1 PETs, it was also important to test whether statistically significant differences existed between the performance of MT1 and MT2/MT3 PETs. Because participating MT1 PETs completed both pre-teaching and post-teaching instruments, comparing the performance of participating MT1 and MT2/MT3 PETs was done by conducting two separate independent-samples $t$-tests: (1) testing for statistically significant differences between MT1 pre-teaching and MT2/MT3 performances; and (2) testing for statistically significant differences between MT1 post-teaching and MT2/MT3 performances. In 
this next sub-section, the pre-teaching performance of MT1 PETs is compared to the performance of MT2/MT3 PETs. Sixteen MT1 PETs completed the pre-teaching instrument. Six MT2 PETs and 16 MT3 PETs completed the paper-and-pencil instrument. Altogether, 22 MT2/MT3 PETs participated.

For every independent-samples $t$-test that was conducted, Cohen's $d$ and $r^{2}$ were also calculated as measures of effect size. The calculation for Cohen's $d$ effect size for independentsamples $t$-test is given below:

$$
d=\frac{\text { Mean }_{1}-\text { Mean }_{2}}{\sqrt{\left(\frac{S D_{1}{ }^{2}}{2}+\frac{S D_{2}{ }^{2}}{2}\right)}}
$$

The interpretation of Cohen's $d$ was discussed above. In addition to calculating Cohen's d effect sizes, $r^{2}$ effect sizes were calculated as an additional measure of the magnitude of effect between groups. The formula for calculating $r^{2}$ is given below:

$$
r^{2}=\frac{t^{2}}{t^{2}+d f}, \text { where } d f \text { are the degrees of freedom. }
$$

The following conventions are used to interpret $r^{2}$ : an effect size of $r^{2}=0.01$ would be considered as "small," an effect size of $r^{2}=0.09$ would be considered as "medium," and an effect size of $r^{2}=0.25$ would be considered as "large."

Comparing MT1 pre-teaching and MT2/MT3 performances. Independent-samples $t$ tests, with $\alpha=0.05$ level of significance, were conducted to test for mean score differences between MT1 pre-teaching scores and MT2/MT3 scores. Levene's test was conducted to test for homogeneity of variances. Levene's test revealed that homogeneity of variances was not met for NP scores, $\mathrm{F}(1,36)=7.90, p=0.008$. The results of the independent-samples $t$-tests test for 
mean score differences between MT1 pre-teaching and MT2/MT3 performances are presented in Table 10.

Table 10

Results of Independent t-tests and Descriptive Statistics MT1 Pre-Teaching vs MT2/MT3

\begin{tabular}{|c|c|c|c|c|c|c|c|c|c|c|}
\hline \multirow{3}{*}{$\begin{array}{l}\text { Score } \\
\text { Type }\end{array}$} & \multicolumn{6}{|c|}{ Group } & \multirow{2}{*}{\multicolumn{2}{|c|}{$\begin{array}{l}\text { 95\% CI for } \\
\text { Mean } \\
\text { Difference }\end{array}$}} & \multirow[b]{3}{*}{$t$} & \multirow[b]{3}{*}{$d f$} \\
\hline & \multicolumn{3}{|c|}{ MT1 Pre-Teaching } & \multicolumn{3}{|c|}{ MT2/MT3 } & & & & \\
\hline & M & SD & $n$ & M & SD & $n$ & $\mathrm{~L}$ & $\mathrm{U}$ & & \\
\hline Overall & 5.74 & 2.28 & 16 & 7.00 & 2.33 & 22 & -0.29 & 2.80 & 1.65 & 36 \\
\hline NPQ & 4.56 & 1.69 & 16 & 5.05 & 1.67 & 22 & -0.63 & 1.61 & 0.89 & 36 \\
\hline UOP & 0.94 & 1.06 & 16 & 2.00 & 1.80 & 22 & 0.04 & 2.09 & $2.11^{*}$ & 36 \\
\hline $\mathrm{NP}^{* * *}$ & 0.19 & 0.54 & 16 & 0.59 & 0.91 & 22 & -0.08 & 0.88 & 1.71 & 34.93 \\
\hline
\end{tabular}

**Note: Welch-Satterthwaite approximation employed due to unequal group variances $* p<.05$

There were statistically significant differences, at the .05 alpha level, between MT1 preteaching and MT2/MT3 performance in UOP scores, but not in the overall score or NPQ and NP scores. Results indicate that MT2/MT3 UOP scores were statistically significantly higher.

Table 11

Cohen's d effect sizes and $r^{2}$ for MT1 pre-teaching scores vs MT2/MT3 scores

\begin{tabular}{|c|c|c|c|c|c|c|c|c|c|}
\hline \multirow[t]{3}{*}{ Score } & \multicolumn{6}{|c|}{ Group } & \multirow{3}{*}{$\begin{array}{l}\text { Max. } \\
\text { Score }\end{array}$} & \multirow{3}{*}{$\begin{array}{c}\text { Cohen's } \\
d\end{array}$} & \multirow{3}{*}{$r^{2}$} \\
\hline & \multicolumn{3}{|c|}{ MT1 Pre-Teaching } & \multicolumn{3}{|c|}{ MT2/MT3 } & & & \\
\hline & $\mathrm{M}$ & $\mathrm{SD}$ & $n$ & M & SD & $n$ & & & \\
\hline Overall & 5.74 & 2.28 & 16 & 7.00 & 2.33 & 22 & 15 & 0.55 & 0.07 \\
\hline NPQ & 4.56 & 1.69 & 16 & 5.05 & 1.67 & 22 & 9 & 0.29 & 0.02 \\
\hline UOP & 0.94 & 1.06 & 16 & 2.00 & 1.80 & 22 & 9 & 0.72 & 0.11 \\
\hline NP & 0.19 & 0.54 & 16 & 0.59 & 0.91 & 22 & 9 & 0.53 & 0.08 \\
\hline
\end{tabular}


There was no statistical difference between MT2/MT3 and MT1 pre-teaching performance in terms of the overall score or NPQ and NP scores. In Table 11, Cohen's $d$ effect sizes and $r^{2}$ are summarized.

Comparing MT1 post-teaching and MT2/MT3 performances. Independent-samples $t$ tests, with $\alpha=0.05$ level of significance, were also conducted to test for mean score differences between MT1 post-teaching scores and MT2/MT3 scores. Levene's test was conducted to test for homogeneity of variances. Levene's test revealed that homogeneity of variances was not met for NP scores, $\mathrm{F}(1,36)=8.47, p=0.006$. The results of the independent-samples $t$-tests test for mean score differences between MT1 post-teaching and MT2/MT3 performances are presented in Table 12.

Table 12

Results of Independent t-tests and Descriptive Statistics MT1 Post-Teaching vs MT2/MT3

\begin{tabular}{|c|c|c|c|c|c|c|c|c|c|c|}
\hline \multirow[t]{3}{*}{$\begin{array}{l}\text { Score } \\
\text { Type }\end{array}$} & \multicolumn{6}{|c|}{ Group } & \multirow{2}{*}{\multicolumn{2}{|c|}{$\begin{array}{l}95 \% \text { CI for } \\
\text { Mean } \\
\text { Difference }\end{array}$}} & \multirow[b]{3}{*}{$t$} & \multirow[b]{3}{*}{$d f$} \\
\hline & \multicolumn{3}{|c|}{ MT1 Post-Teaching } & \multicolumn{3}{|c|}{ MT2/MT3 } & & & & \\
\hline & $\mathrm{M}$ & SD & $n$ & M & SD & $n$ & $\mathrm{~L}$ & $\mathrm{U}$ & & \\
\hline Overall & 7.14 & 2.13 & 16 & 7.00 & 2.33 & 22 & -1.65 & 1.36 & -0.20 & 36.00 \\
\hline NPQ & 4.97 & 1.52 & 16 & 5.05 & 1.67 & 22 & -0.99 & 1.16 & 0.16 & 36.00 \\
\hline UOP & 2.88 & 1.59 & 16 & 2.00 & 1.80 & 22 & -2.02 & 0.27 & -1.55 & 36.00 \\
\hline $\mathrm{NP}^{* *}$ & 1.44 & 1.63 & 16 & 0.59 & 0.91 & 22 & -1.78 & 0.09 & $-1.87^{*}$ & 21.73 \\
\hline
\end{tabular}

**Note: Welch-Satterthwaite approximation employed due to unequal group variances $* p<.05$

There were statistically significant differences, at the .05 level of significance, between MT1 post-teaching and MT2/MT3 performance in NP scores, but not in the overall score or NPQ and UOP scores. Results indicate that MT1 post-teaching NP scores were statistically significantly higher. There was no statistical difference between MT2/MT3 and MT1 post- 
teaching performance in terms of the overall score or NPQ and UOP scores. In Table 13, Cohen's $d$ effect sizes and $r^{2}$ are summarized.

Table 13

Cohen's d effect sizes and $r^{2}$ for MT1 post-teaching scores vs MT2/MT3 scores

\begin{tabular}{|c|c|c|c|c|c|c|c|c|c|}
\hline \multirow[t]{3}{*}{ Score } & \multicolumn{6}{|c|}{ Group } & \multirow{3}{*}{$\begin{array}{l}\text { Max. } \\
\text { Score }\end{array}$} & \multirow{3}{*}{$\begin{array}{c}\text { Cohen's } \\
d\end{array}$} & \multirow{3}{*}{$r^{2}$} \\
\hline & \multicolumn{3}{|c|}{ MT1 Post-Teaching } & \multicolumn{3}{|c|}{ MT2/MT3 } & & & \\
\hline & $\mathrm{M}$ & SD & $n$ & $\mathrm{M}$ & SD & $n$ & & & \\
\hline Overall & 7.14 & 2.13 & 16 & 7.00 & 2.33 & 22 & 15 & 0.06 & 0.001 \\
\hline NPQ & 4.97 & 1.52 & 16 & 5.05 & 1.67 & 22 & 9 & 0.05 & 0.001 \\
\hline UOP & 2.88 & 1.59 & 16 & 2.00 & 1.80 & 22 & 9 & 0.52 & 0.06 \\
\hline NP & 1.44 & 1.63 & 16 & 0.59 & 0.91 & 22 & 9 & 0.64 & 0.14 \\
\hline
\end{tabular}

\section{Summary and Concluding Comments}

This chapter has reported on the results of this study's data. This dissertation study involved the collection and analysis of both quantitative and qualitative data, using paper-andpencil instruments and verbal interviews. Participating PETs' written responses to tasks on the paper-and-pencil instrument provided qualitative data which were examined for correctness and strategies employed. The transcribed text of the four audio-taped interviews with participating interviewees provided additional qualitative data.

The qualitative analysis of pre- and post-teaching data revealed that the instructional intervention was not as successful as desired. Participating MT1 PETs demonstrated only trivial knowledge of any of the properties at the pre-teaching stage. For some MT1 participants, their knowledge of the associative and commutative properties improved between the pre- and postteaching stages but many of them still confused the names of the associative properties with those of the commutative properties. With respect to questions framed with commutative and 
associative properties in mind, more participants at the post-teaching stage than at the preteaching stage recognized and responded to these questions in a manner that demonstrated they were aware of the mathematical objects under consideration. With respect to questions framed with the distributive property in mind, none or nearly none of the participants appropriately responded to "distributive property" signs at the pre-teaching stage; at the post-teaching stage, the number of participants appropriately responding to "distributive property" signs was extremely low as to be negligible. For nearly all the participants, their concept images of the distributive property did not improve in meaningful ways.

Most of the participating MT2/MT3 PETs demonstrated very little knowledge or understanding of properties. With respect to questions framed with commutative and associative properties in mind, some MT2/MT3 participants recognized and appropriately responded to the signs. Even so, none demonstrated well-formed concept images of the properties. Some of the MT2/MT3 participants confused the names of the associative properties with those of the commutative properties. Others used the associative property for multiplication to evaluate $(72 \times$ $5) \times 2$ but did not use the same property to evaluate $\left(120 \times \frac{1}{32}\right) \times 64$. This was also observed among MT1 participants even after participating in lessons emphasizing properties.

The PETs' performances on the paper-and-pencil instruments provided quantitative data. Pre- and post-teaching performance of participating MT1 PETs was compared using repeatedmeasures $t$-tests. Independent $t$-tests were conducted to test whether MT1 PETs' performance was statistically significantly different from MT2/MT3 PETs’ performance.

The quantitative analysis of pre- and post-teaching data complemented the qualitative analysis, both suggesting the instructional intervention had not been as successful as expected. The results of the repeated-measures $t$-tests indicate that the overall performance of participating 
MT1 PETs improved after participating in the instructional intervention. Cohen's $d$ effect sizes (Cohen, 1988) showed that the instructional intervention had a moderately large effect on the overall score $(d=0.65)$, a small effect on the NPQ score $(d=0.25)$, and a large effect on both the UOP score $(d=1.50)$ and the NP score $(d=1.09)$. The results show that the performance of participating MT1 PETs improved after participating in lessons emphasizing properties but were the improvements educationally significant?

Performance improvement ratios (PIRs) were calculated as a measure for quantifying the effect of teaching in MT1. It would have been desired to observe PIRs of at least 50\%. If such PIRs were observed for any of the scores, it would have suggested that mean gain for the score was at least $50 \%$ of the maximum possible gain achievable. The overall score mean gain was $16 \%$ of the maximum possible gain achievable. The NPQ score mean gain was $9 \%$ of the maximum possible gain achievable. The UOP score mean gain was $25 \%$ of the maximum possible gain achievable. The NP score mean gain was $15 \%$ of the maximum possible gain achievable. Even though large effect sizes were observed, the corresponding PIRs were relatively low. This implies that improvements in the performance of MT1 PETs were not educationally significant. The instructional intervention emphasizing properties had not been as successful as desired.

Independent-samples $t$-tests, with $\alpha=0.05$ level of significance, were conducted to test for mean score differences between MT1 pre-teaching scores and MT2/MT3 scores. There were statistically significant differences, at the .05 level of significance, between MT1 pre-teaching and MT2/MT3 performance in UOP scores, but not in the overall score or NPQ and NP scores. There was no statistical difference between MT2/MT3 and MT1 pre-teaching performance in terms of the overall score or NPQ and NP scores. The magnitude of effect (Cohen's $d$ effect sizes 
and $r^{2}$ ) between MT2/MT3 and MT1 pre-teaching performances was moderate for the overall scores $\left(d=0.55, r^{2}=.07\right)$ and NP scores $\left(d=0.53, r^{2}=0.08\right)$, small for the NPQ scores $(d=$ $\left.0.29, r^{2}=0.02\right)$, and large for the UOP scores $\left(d=0.72, r^{2}=0.11\right)$. It is important to note that small sample sizes may yield large effect sizes but no practical significance to those effect sizes.

To test for mean score differences between MT1 post-teaching scores and MT2/MT3 scores, independent-samples $t$-tests, with $\alpha=0.05$ level of significance, were conducted. There were statistically significant differences, at the .05 level of significance, between MT1 postteaching and MT2/MT3 performance in NP scores, but not in the overall score or NPQ and UOP scores. There was no statistical difference between MT2/MT3 and MT1 post-teaching performances in terms of the overall score or NPQ and UOP scores. The magnitude of effect (Cohen's $d$ effect sizes and $r^{2}$ ) between MT2/MT3 and MT1 post-teaching performances was very small for the overall scores $\left(d=0.06, r^{2}=.001\right)$ and NPQ scores $\left(d=0.05, r^{2}=0.001\right)$, moderate for the UOP scores $\left(d=0.52, r^{2}=0.06\right)$, and moderately large for the NP scores $(d=$ $\left.0.64, r^{2}=0.14\right)$

In summary, both the quantitative and qualitative analyses revealed that the participating PETs did not demonstrate strong knowledge of properties. The analyses revealed that there was not an educationally significant difference between MT1 and MT2/MT3 participants' knowledge of number of properties. The analyses also revealed that the instructional intervention emphasizing properties did not yield educationally significant gains in MT1 PETs' knowledge of properties. In the next and final chapter of this dissertation, answers to research questions are presented, implications of this study are discussed, and recommendations for further study and comments on this study's limitations are considered and discussed as well. 


\section{CHAPTER V: DISCUSSION AND CONCLUDING REMARKS}

In this final chapter, quantitative and qualitative findings are drawn together and answers to the research questions are given. Then, implications of this study are discussed, and recommendations for further study and comments on this study's limitations are considered and stated. Both the quantitative and qualitative analyses revealed that the participating PETs did not demonstrate strong knowledge of properties of numbers. The analyses revealed that the differences between MT1 and MT2/MT3 participants' knowledge of properties were not educationally significant.

\section{Answers to Research Question 1}

Question 1: What knowledge of properties of real numbers did PETs have?

A major goal of this dissertation was to ascertain the knowledge of participating PETs with respect to properties of real of numbers. Qualitative analysis of verbal interviews, and of PETs' written responses to the paper-and-pencil instruments, were studied to answer Research Question 1. To address this research question appropriately, the question was separated into the following four parts:

a) What knowledge of the commutative property for addition/multiplication did participating PETs demonstrate?

b) What knowledge of the associative property for addition/multiplication did participating PETs demonstrate?

c) What knowledge of the distributive property did participating PETs demonstrate?

d) What knowledge of inverse and identity properties did participating PETs demonstrate? 


\section{Answer to Question 1a-PETs' Knowledge of Commutative Properties}

A well-defined study on prospective teachers' knowledge should not just report on what PETs do not know, it should also inform on what they do know. Participating PETs exhibited knowledge relating to the commutativity of addition and multiplication. MT2/MT3 PETs made comments such as “order doesn't matter" with respect to addition and multiplication. MT1 PETs also demonstrated knowledge of this idea even before participating in the instructional intervention. Even though participating PETs demonstrated knowledge of the idea that "order doesn't matter" when adding and multiplying, they did not know the formal language “commutative property for addition" and "commutative property for multiplication."

There were many participating PETs who conflated the commutative and associative properties. This conflation was observed among MT1 PETs even after participating in the instructional intervention. Even though participating PETs were aware that changing the order of the operands in a sum or product did not affect the result, they did not seem to have knowledge of how this concept could be useful in evaluating expressions such as " $101+12+99$ " or " $5 \times 6$ $\times 1 / 5$." Superficial understanding of commutativity — knowing only that for addition and multiplication changing the order of the operands is permissible - is not sufficient and hinders the development of well-formed conceptual images of commutative properties in simplifying arithmetic calculations (Anthony \& Walshaw, 2002; Kanbir, 2016; Kanbir et al., 2018; Warren \& English, 2000).

Participating PETs were inclined to reason procedurally rather than conceptually with regards to the commutative properties for addition/multiplication. When presented with signs such as " $16+26=x+16$ " and " $13 \times y=9 \times 13$," most participating PETs did not explicitly demonstrate that they understood the mathematical objects under consideration were the 
commutative properties for addition and for multiplication, respectively. Most did not use the words "commutative property," "commutative property for addition" or "commutative property for multiplication.”

\section{Answer to Question 1b-PETs' Knowledge of Associative Properties}

Some MT2/MT3 PETs exhibited knowledge of the idea that the parentheses in expressions such as "940 $+(60+427)$ " or " $(72 \times 5) \times 2$ " can be ignored when evaluating the expressions, but they did not connect this knowledge to the associative properties for addition/multiplication. Most MT1 PETs did not exhibit knowledge of this idea prior to participating in the instructional intervention. It was not just important to know that the parentheses can be ignored in such expressions, it was also important to understand why ignoring the parentheses did not affect the sum or product by making connections with the concepts of addition and multiplication associativity. Difficulties in attaining well-developed knowledge of the associative and commutative properties can be attributed to imprecise and informal understanding of these properties and their applications (Kanbir, 2016; Kanbir et al., 2018; Larsen, 2010).

Participating PETs showed preference for reasoning procedurally rather than conceptually with regards to the associative properties for addition/multiplication. When presented with signs such as "940+ $(60+427)$ " or “ $(72 \times 5) \times 2$," most participating PETs did not explicitly demonstrate that they recognized the mathematical objects under consideration were the associative properties for addition and for multiplication, respectively. Most did not use the words "associative property," "associative property for addition" or "associative property for multiplication." 
Many MT2/MT3 PETs did not demonstrate appropriate expressive understandings that associating 940 and 60 in $940+(60+427)$ and associating 5 and 2 in $(72 \times 5) \times 2$ superseded using the rules for order of operations. Among MT1 PETs, more participants at the post-teaching stage than at the pre-teaching stage demonstrated correct expressive understandings of associativity for addition and multiplication operations. However, as reported above, conflation between the names of associative and commutative properties persisted even after participating in the instructional intervention. This conflation was not unique to MT1 PETs because it was also observed among MT2/MT3 PETs.

\section{Answer to Question 1c-PETs' Knowledge of the Distributive Property}

Participating PETs did not demonstrate appropriate receptive or expressive knowledge or understandings of the distributive property and its applications. With respect to signs for which the mathematical "object" was the distributive property, virtually none of the participating MT1 PETs responded appropriately at pre-teaching stage; at the post-teaching teaching, the number of MT1 PETs that recognized and responded to "distributive property" signs was extremely small. Participating in the instructional intervention did not help MT1 participants improve their concept images of the distributive property in meaningful ways. Participating MT1 interviewees provided appropriate examples of the distributive property during both their pre-teaching and post-teaching interviews but did not recognize arithmetic patterns, such as $0.8 \times 315+315 \times 0.2$, for which using the distributive property would be appropriate, even after participating in the instructional intervention.

Most of the participating MT2/MT3 PETs also demonstrated very little knowledge or understanding of distributive property and its applications. Some of them had imprecise memories of the distributive property but lacked accurate expressive knowledge of the property 
and did not recognize the property's usefulness when responding to signs such as " $3 x=21 \times 3+$ $4 \times 3$ " or " $0.6 \times 250+250 \times 0.4$." There were some participating PETs who recognized the role of the distributive property in tasks such as " $0.6 \times 250+250 \times 0.4$ " and " $20 \times(10+5)=(20 \times$ $10)+(20 \times y)$," but did not explicitly state that they using the distributive property. They did not

use the words "distributive property," or they may have given an incorrect name. Knowledge of the actual names of the properties being used is important. "Consider what might happen, for example, if the teacher were to be talking about the associative property for multiplication, or the distributive property, but the student mixed the labels" (Kanbir, 2016, p. 156). The might lead the student not understand what the teacher was saying.

\section{Answer to Question 1d-PETs' Knowledge of Inverse and Identity Properties}

Participating interviewees were asked to describe the meanings of the following terms during their interviews: additive identity, additive inverse, multiplicative identity, and multiplicative inverse. MT2/MT3 interviewees demonstrated practically no knowledge of the meanings of any of those terms. They could not recall learning anything about any of the identity or inverse properties. They made guesses about what the terminology meant but none provided appropriate descriptions.

MT1 interviewees also did not demonstrate strong understanding of the meanings of these aforementioned terms in either pre- or post-teaching interviews. Neither of the two MT1 interviewees could provide appropriate descriptions of any of the identity or inverse properties during the pre-teaching interview. One of the MT1 interviewees, Betty, stated that she had not previously seen, or been taught the meaning of identity or inverse properties. At the postteaching stage, Betty was able to describe the meanings of the terms "additive inverse" and 
"multiplicative identity" but could not describe the meaning of "additive identity" and gave an incorrect description of "multiplicative inverse."

The other MT1 interviewee, Ally, was not able to provide appropriate descriptions of any of the identity or inverse properties during the post-teaching interview. This interviewee could not recall from memory anything she had ever learned about identity or inverse properties. The interviewee demonstrated some knowledge of the concept of an inverse but did not exhibit any well-formed knowledge or imagery of the additive inverse property or the multiplicative inverse property.

\section{Answers to Research Question 2}

Question 2: How does the PETs' understanding of number properties prior to a teaching intervention compare to the PETs' understanding of number properties after a teaching intervention?

With respect to participating MT1 PETs, part of the goal was to describe their knowledge of properties before and after participating in lessons emphasizing properties of real numbers. Participating MT1 PETs demonstrated only trivial knowledge of any of the properties at the preteaching stage. For some MT1 participants, their knowledge of the associative and commutative properties improved between the pre- and post-teaching stages but many of them still confused the names of the associative properties with those of the commutative properties. At the preteaching stage, none of the MT1 participants recognized signs for which the mathematical objects were the commutative properties for addition and for multiplication. At the post-teaching stage, six MT1 participants recognized and appropriately responded to "commutative property" signs. 
To help learners of mathematics understand the concept of commutativity, the commutative properties must not be taught superficially (Hurst, 2017; Larsen, 2010). I made efforts to ensure that I was not just pushing PETs to superficially remember that for addition and multiplication sentences changing the order of the operands does not change the result. Commutativity of addition was introduced and discussed by exploring various sums, such as $5+$ 3 and $3+5$, on a number line and making judgements about the nature of addition.

\section{Classroom Tasks (Whole Number Addition)}

DIRECTIONS: Get into groups of three or four and work on the following tasks. Discuss the questions, work out what is asked, determine the meaning of each, and answer appropriately.

8. Use a number line to find the sum of $5+3$ and $3+5$. What do you find? What can you conjecture about the addition of whole numbers?

- Let $a$ and $b$ be two unique whole numbers. Use a number line to find the sum of $a+b$ and $b+a$ ? What do you find? Repeat this previous step a few more times with different numbers for $a$ and $b$. What can you conclude about the addition of whole numbers?

9. What would be a quick way of determining the value of $99+234+1$ without using a calculator or writing anything down? What did you do and is it related to what you concluded about the addition of whole numbers from above?

10. If $a=12, b=6$ and $c=2$, what are the values of each of the following? $(a+b)+c$ $a+(\mathrm{b}+c)$

- Repeat this, only this time let $a=51, b=26$, and $c=12$. What do you find? What can you conjecture about the addition of whole numbers?

Anthony and Walshaw (2002) and Warren and English (2000) argued that commutative properties are typically taught procedurally and young learners are not provided learning opportunities to understand why interchanging operands does not affect the sum or product. In the MT1 classroom, PETs explored various sums, such as $5+3$ and $3+5$, on a number line and were asked to observe the results they obtained and conjecture what it meant about whole 
number addition. Other tasks, as shown above, we included in the lesson to encourage PETs to think about and explore the commutativity nature of addition, as well as the associativity nature of addition. As the semester progressed, the number system was expanded to include integers, then rational numbers, and then finally the set of real numbers. As we expanded the number system, PETs were challenged to examine whether the properties we found, or agreed to, about whole numbers could be extended to the set of integers, then rational numbers, and then finally the set of real numbers.

Commutativity of multiplication was introduced through the various way of modeling multiplication. MT1 PETs explored various products, such as $5 \times 3$ and $3 \times 5$, using different models: repeated addition, array/area, and skip counting. As the semester progressed and the number system was expanded, PETs were challenged to examine whether what we found, or agreed to, about whole numbers could be extended to the set of integers, then rational numbers, and then finally the set of real numbers.

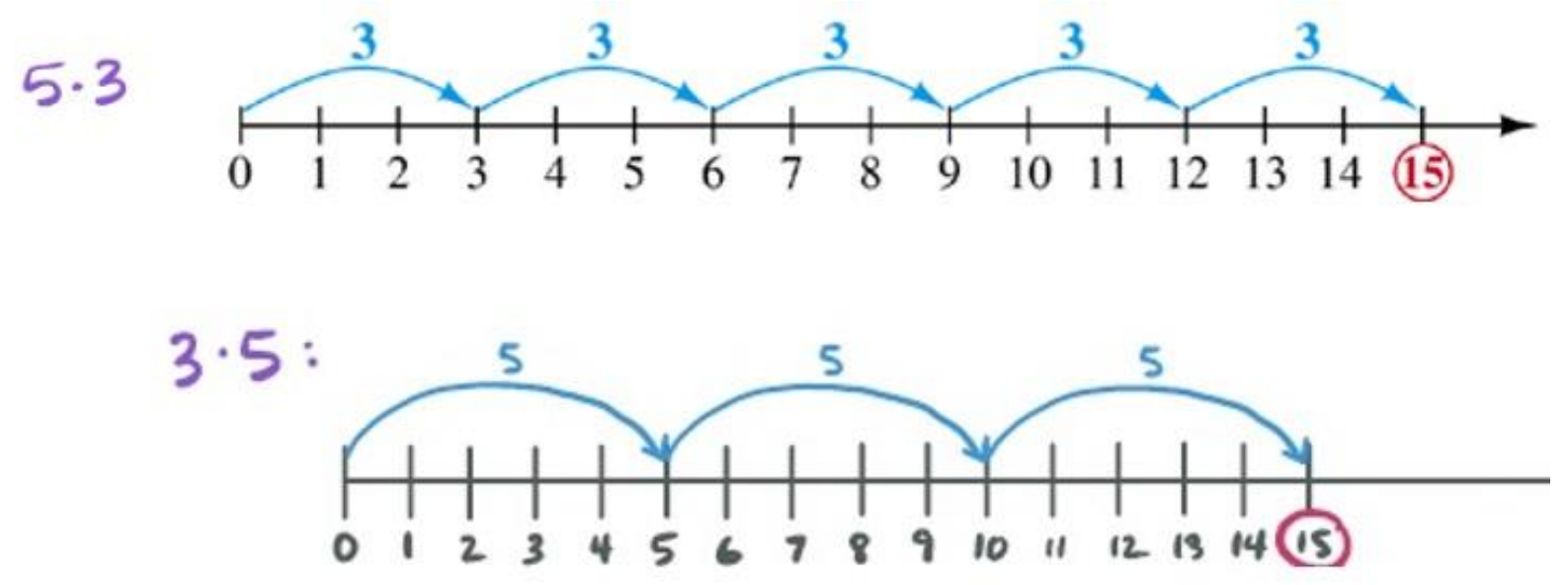

Figure 6. Skip-count models for $5 \times 3$ and $3 \times 5$.

The goal was provide learning opportunities for MT1 PETs to develop understanding of the commutative properties that was deeper than memorization of the definitions of the properties. MT1 PETs were provided opportunities to learn how commutative properties could 
be used to simplify arithmetic calculations such as $99+234+1$ or $2 \times 29 \times 5$. These efforts of emphasizing properties in lessons did not yield educationally significant improvements in PETs' concept images of the commutative properties. Both the quantitative and qualitative analysis of pre- and post-teaching data revealed that the instructional intervention had not been as successful as desired. I posit that the time constraints of packing the intervention in one semester did not allow participating MT1 PETs enough time to interact with, and appreciate the power of, these properties in simplifying, evaluating, interpreting arithmetic expressions.

With respect to questions framed with the associative properties in mind, more MT1 participants on the post-teaching instrument than on the pre-teaching instrument recognized and responded to those questions in a manner which showed that they understood the mathematical objects under consideration. However, most participating PETs' knowledge did not improve in educationally significant ways between the pre-teaching and post-teaching stages. During classroom instruction and discussion, efforts were made to introduce and discuss the associative properties in educationally meaningfully ways during lessons on whole number operations (see class handouts in Appendix F). And, as mentioned above, as the semester progressed and the number system was expanded, PETs were challenged to examine whether the properties we learned, and agreed to, about whole numbers could be extended to the set of integers, then rational numbers, and then finally the set of real numbers. There was some evidence of positive changes in the concept images of a handful of MT1 PETs with respect to the associative and commutative properties. Even so, I observed those in this group use the associative property for multiplication when computing $(36 \times 5) \times 2$ but not when computing $48 \times\left(\frac{1}{24} \times 150\right)$. This may point to another issue of PETs' apprehension when working with fractions (Desta, 2019). 
With respect to signs for which the mathematical "object" was the distributive property, virtually none of the participating MT1 PETs responded appropriately at the pre-teaching stage, and hardly any improvement was observed between the pre-teaching and post-teaching stages. When presented with questions framed with the distributive property in mind, MT1 PETs showed preference for procedural calculations heavily dependent on order of operations. MT1 interviewees, Ally and Betty, for example, gave acceptable examples of the distributive property in both pre- and post-teaching interviews but did not demonstrate well-formed concept images of this property, even after participating in the instructional intervention. Ally, for example, used factoring when solving for $x$ in $5.25 x=70 \times 5.25+30 \times 5.25$ but did not use the words “distributive property," and Betty recognized that $84 \times 5=80 \times 5+4 \times 5$ but incorrectly used the words "associative property." Ally and Betty both exhibited correct expressive knowledge of some applications of the distributive property but did not connect their work in explicit terms to the distributive property. Because neither Ally nor Betty's concept image of the distributive was well-developed, neither of them recognized or responded appropriately to arithmetic patterns, such as $0.8 \times 315+315 \times 0.2$, for which using the distributive property was appropriate.

During classroom instruction and discussion, efforts were made to introduce and discuss the distributive property in educationally meaningfully ways during lessons on whole number operations (see class handouts in Appendix F). And then as the semester progressed and the number system was expanded, PETs were challenged to examine whether the distributive property could be extended and be applied to the set of integers, rational numbers, and the set of real numbers. 


\section{Classroom Tasks (Whole Number Multiplication \& Properties)}

DIRECTIONS: Get into groups of three or four and work on the following tasks. Discuss the questions, work out what is asked, determine the meaning of each, and answer appropriately.

6. Without using a calculator or relying on any algorithm, compute each of the following products in at least three different ways. State any properties that you use.
a. $24 \times 25$
b. $120 \times 325$

7. How could you use the stated property to evaluate $12 \times 250$ ?

a. How could you use an associative property to find the value of $12 \times 250$ ?

b. How could you use the distributive property to find the value of $12 \times 250$ ?

8. How could you use the distributive property to find the value of $17 \times 97+17 \times 3$ ?

9. Which property would you use to find the value of $p$ ? $16 \times 28=11 \times 28+p \times 28$.

10. How would you quickly determine the value of $25 \times 37 \times 4$ ? Which property (or properties) would you be applying?

\section{Table 14}

Pre-T and Post-T Responses to NPQs

\begin{tabular}{|c|c|c|c|c|c|c|}
\hline Property & Q\# & & & $\begin{array}{l}\text { No. of Correct } \\
\text { Responses (\%) }\end{array}$ & $\begin{array}{l}\text { No. Relying on } \\
\text { Property (\%) }\end{array}$ & $\begin{array}{l}\text { No. Naming } \\
\text { Property }(\%)\end{array}$ \\
\hline \multirow{2}{*}{$\begin{array}{l}\text { Commutative } \\
\text { Property for + }\end{array}$} & \multirow{2}{*}{ Q9 } & Pre-t & $16+26=x+16$ & $(94 \%)$ & $(0 \%)$ & $0 \quad(0 \%)$ \\
\hline & & Post-t & $36+18=x+36$ & $(94 \%)$ & $(38 \%)$ & $(44 \%)$ \\
\hline \multirow{2}{*}{$\begin{array}{l}\text { Commutative } \\
\text { Property for } x\end{array}$} & \multirow{2}{*}{ Q3 } & Pre-t & $13 \times y=9 \times 13$ & $(81 \%)$ & $(0 \%)$ & $(0 \%)$ \\
\hline & & Post-t & $15 \times y=5 \times 15$ & $(94 \%)$ & $(38 \%)$ & $(38 \%)$ \\
\hline \multirow{2}{*}{$\begin{array}{c}\text { Associative } \\
\text { Property for }+\end{array}$} & \multirow{2}{*}{ Q2 } & Pre-t & $940+(60+427)$ & $(88 \%)$ & $(31 \%)$ & $(6 \%)$ \\
\hline & & Post-t & $920+(80+533)$ & $14 \quad(88 \%)$ & $(69 \%)$ & $(19 \%)$ \\
\hline \multirow{4}{*}{$\begin{array}{l}\text { Associative } \\
\text { Property for } x\end{array}$} & \multirow{2}{*}{ Q5 } & Pre-t & $(72 \times 5) \times 2$ & $(94 \%)$ & $(19 \%)$ & $(6 \%)$ \\
\hline & & Post-t & $(36 \times 5) \times 2$ & $(88 \%)$ & $(63 \%)$ & $(31 \%)$ \\
\hline & \multirow{2}{*}{ Q10 } & Pre-t & $(120 \times(1 / 32)) \times 64$ & $(13 \%)$ & $(13 \%)$ & $(6 \%)$ \\
\hline & & Post-t & $48 \times((1 / 24) \times 150)$ & $(31 \%)$ & $(31 \%)$ & $(13 \%)$ \\
\hline \multirow{8}{*}{$\begin{array}{c}\text { Distributive } \\
\text { Property }\end{array}$} & \multirow{2}{*}{ Q4 } & Pre-t & $3 x=21 \times 3+4 \times 3$ & $(56 \%)$ & $(6 \%)$ & $(0 \%)$ \\
\hline & & Post-t & $3 x=21 \times 3+4 \times 3$ & $(63 \%)$ & $(0 \%)$ & $(0 \%)$ \\
\hline & \multirow{2}{*}{ Q7 } & Pre-t & $0.6 \times 250+250 \times 0.4$ & $(31 \%)$ & $(0 \%)$ & $(0 \%)$ \\
\hline & & Post-t & $0.8 \times 315+315 \times 0.2$ & $(13 \%)$ & $(13 \%)$ & $(0 \%)$ \\
\hline & \multirow{2}{*}{ Q8 } & Pre-t & $\begin{array}{l}20 \times(10+5) \\
=(20 \times 10)+(20 \times y)\end{array}$ & $(63 \%)$ & $1 \quad(6 \%)$ & $0 \quad(0 \%)$ \\
\hline & & Post- $\mathrm{t}$ & $\begin{array}{l}50 \times(10+5) \\
=(50 \times 10)+(50 \times y)\end{array}$ & $11 \quad(69 \%)$ & $(38 \%)$ & $(19 \%)$ \\
\hline & \multirow{2}{*}{ Q11 } & Pre-t & $(1 / 5) \times 96+(1 / 5) \times 4$ & $(0 \%)$ & $(0 \%)$ & $(0 \%)$ \\
\hline & & Post-t & $(1 / 5) \times 96+(1 / 5) \times 4$ & $(6 \%)$ & $(0 \%)$ & $0 \quad(0 \%)$ \\
\hline
\end{tabular}


In summary, with respect to the properties of operations under consideration, participating MT1 PETs did not exhibit strong receptive or expressive understanding. Participating in the instructional intervention resulted only in modest gains in positively influencing participating PETs' concept images of properties of operations. The intervention did not result in qualitatively significant improvements in PETs' cognitive structures, or concept images, as had been desired and expected. MT1 PETs' responses to number property questions (NPQs) are summarized in Table 14 above.

\section{Answer to Research Question 3}

Question 3: How does the understanding of number properties of PETs in a course focused on number properties compare to the understanding of number properties of PETs who previously took the course?

MT2/MT3 did not participate in lessons emphasizing properties because that content is covered in the MT1 course. The goal was to ascertain what they knew and understood about structural properties based on their knowledge at the time they responded to the paper-and-pencil instruments and interviews. With respect to signs for which the underlying mathematical objects were the associative properties for addition and for multiplication, some MT2/MT3 participants recognized the signs and appropriately responded but not all who did so used the words "associative property," "associative property for addition" or "associative property for multiplication." Some of the MT2/MT3 participants confused the names of the associative properties with those of the commutative properties. Others used the associative property for multiplication to evaluate $(72 \times 5) \times 2$ but did not use the same property to evaluate $\left(120 \times \frac{1}{32}\right.$

) $\times 64$. Overall, only a few of the MT2/MT3 participants exhibited some knowledge of the 
associative properties, and even those few rarely demonstrated well-formed concept images of the associative properties.

With respect to signs for which the underlying mathematical object was the distributive property, no MT2/MT3 participant demonstrated a strong knowledge of the underlying mathematical object. For some of the "distributive property" questions, a handful of the participants recognized the concept of factoring or distributing multiplication over addition, but nearly none of them used the words "distributive property." They did not exhibit well-formed concept images of the distributive property. Practically none of them recognized arithmetic patterns, such as $315 \times 0.8+315 \times 0.2$, for which using the distributive property would be appropriate.

MT2/MT3 PETs' responses to number property questions (NPQs) are summarized in Table 15. Most MT2/MT3 participants did not demonstrate strong knowledge of properties of real numbers. They did not demonstrate exhibit well-formed concept images, or cognitive structures, of any of the commutative properties (for addition and for multiplication), associative properties (for addition and for multiplication), or the distributive property (for multiplication over addition).

There appeared to be a discernable qualitative difference between MT1 PETs' preteaching knowledge and MT2/MT3 PETs' knowledge of properties. There were more MT2/MT3 PETs recognizing and appropriately responding to "number property" signs than there were MT1 PETs doing the same at the pre-teaching stage (see Table 14 and Table 15 for comparison and contrast). MT1 PETs made modest gains with respect to their knowledge of number properties between the pre-teaching and post-teaching stages. However, as noted earlier, the gains were not educationally significant and did not result in qualitatively significant improvements in MT1 
PETs' concept images, or cognitive structures, of any of the commutative properties (for addition and for multiplication), associative properties (for addition and for multiplication), or the distributive property (for multiplication over addition). MT2/MT3 PETs' knowledge of properties was more comparable to the post-teaching knowledge of MT1 PETs than their preteaching knowledge.

Table 15

MT2/MT3 Responses to the NPQs

\begin{tabular}{|c|c|c|c|c|c|}
\hline Property & Q\# & & $\begin{array}{l}\text { No. of Correct } \\
\text { Responses (\%) }\end{array}$ & $\begin{array}{l}\text { No. Relying on } \\
\text { Property (\%) }\end{array}$ & $\begin{array}{l}\text { No. Naming } \\
\text { Property (\%) }\end{array}$ \\
\hline $\begin{array}{l}\text { Commutative } \\
\text { Property for }+\end{array}$ & Q9 & $16+26=x+16$ & $19(86 \%)$ & $6(27 \%)$ & $5(23 \%)$ \\
\hline $\begin{array}{l}\text { Commutative } \\
\text { Property for } x\end{array}$ & Q3 & $13 \times y=9 \times 13$ & $19(86 \%)$ & $6(27 \%)$ & $6(27 \%)$ \\
\hline $\begin{array}{c}\text { Associative } \\
\text { Property for }+\end{array}$ & Q2 & $940+(60+427)$ & $14(64 \%)$ & $10(45 \%)$ & $1(5 \%)$ \\
\hline Associative & Q5 & $(72 \times 5) \times 2$ & $18(82 \%)$ & $8(36 \%)$ & $1(5 \%)$ \\
\hline Property for $x$ & Q10 & $(120 \times(1 / 32)) \times 64$ & $3(14 \%)$ & $3(14 \%)$ & $0(0 \%)$ \\
\hline \multirow{4}{*}{$\begin{array}{c}\text { Distributive } \\
\text { Property }\end{array}$} & Q4 & $3 x=21 \times 3+4 \times 3$ & $17(77 \%)$ & $0(0 \%)$ & $0(0 \%)$ \\
\hline & Q7 & $0.6 \times 250+250 \times 0.4$ & $11(50 \%)$ & $3(14 \%)$ & $1(5 \%)$ \\
\hline & Q8 & $\begin{array}{l}20 \times(10+5) \\
=(20 \times 10)+(20 \times y)\end{array}$ & $19(86 \%)$ & $6(27 \%)$ & $2(9 \%)$ \\
\hline & Q11 & $(1 / 5) \times 96+(1 / 5) \times 4$ & $5(23 \%)$ & $1(5 \%)$ & $0(0 \%)$ \\
\hline
\end{tabular}

\section{Concluding Remarks}

According to the Standards documents (NCTM, 1989, 2000, 2006; NGA \& CCSSO, 2010), by the time students are in the middle-school grades they are expected to recognize and be 
able to apply properties of real numbers to solve or simplify mathematical expressions. Kanbir et al. (2018) wrote that the implemented curriculum on structural properties of real numbers might not be occurring in the elementary and middle-school grades as recommended by the Standards documents. They found that the seventh-grade students in their study hardly demonstrated any knowledge of the associative and distributive properties prior to participating in their study's teaching intervention. Prior to participating in the teaching intervention, seventh-grade students in the Kanbir et al. (2018) study could not recognize or apply the associative or distributive properties in simplifying expressions. Similarly, participating preservice elementary teachers in this dissertation study also did not demonstrate strong knowledge of structural properties of real numbers, and that remained the case for most of those who participated in instruction emphasizing properties of real numbers. Most of them were not aware of the usefulness of the properties in simplifying numerical expressions or solving algebraic equations.

Preservice teachers in this study did not have strong knowledge of properties of real numbers even though they completed their elementary and secondary education in the United States of America during the Standards era (NCTM, 1989, 2000, 2006; NGA \& CCSSO, 2010). This supports Kanbir and colleagues' (Kanbir et al., 2018) claim that the "intended curriculum had not had the effect that was desired by those who prepared the Standards documents" (Monandi, 2018, p. 11). Ellerton and Clements (2011) contended that part of the issue could be attributed to inadequate preparation of elementary and middle-school teachers. Poor preparation of teachers to teach early algebra makes young learners vulnerable to failure (Blanton et al., 2015), and the solution is for teacher education programs to provide preservice teachers with sufficient support and learning opportunities for them to meet the current standards for teaching early algebra. 
The findings in this dissertation study collaborate the results of Barnett and Ding (2019), Ding (2016) and Ding et al. (2013). Barnett and Ding (2019) investigated the teaching of the associative property for addition and for multiplication in a natural classroom setting. They found the associative properties and the commutative properties were often conflated. They found that the associative properties were not presented with substantial explanation or representation in most classrooms and were mostly mentioned in the context of computation strategies. Few classrooms taught the associative properties with rich concrete representations. Ding et al. (2013) examined preservice elementary teachers' knowledge for teaching the associative property for multiplication and found that many of the preservice elementary teachers in their studies did not possess a conceptually-rich understanding of the associative property for multiplication, and many of them confused the associative properties with commutative properties.

\section{Limitations of the Study}

This study generated important data related to early algebra and preservice teachers' knowledge of content they will eventually be expected to teach. However, there are certain limitations that must be acknowledged. First, this study was conducted with non-random samples, and the number of participants selected for interviews was very small. Second, because this study was conducted with a non-random samples, this study's findings are not generalizable to any well-defined population of preservice elementary-school mathematics teachers. Even though the findings are not generalizable, they reveal important information about what the participating preservice teachers knew and understood regarding properties of real numbers. I contend that the observations made in this study are not unique to the group of preservice teachers who participated in this study; similar observations regarding preservice teachers' 
knowledge of properties are likely to also be observed among any other group of preservice elementary- and middle-school teachers.

Other limitations of this study include the constraints associated with the implementation of the teaching intervention. This study was centered on three mathematics courses for teachers offered at a two-year community college. The teaching intervention occurred in the first of these three courses. To form and maintain articulation agreements with four-year institutions to allow for a seamless transfer process for its students, this community college-whose courses and students are subject in this investigation-requires its courses to go through a formal process of approval and have a syllabus and course schedule recorded with the college's curriculum committee. Rules regarding deviations from the approved syllabus and course schedule constrained me from making major changes to the course schedule for MT1. Instead, the teaching intervention in the MT1 course involved giving properties of real numbers an elevated focus during instruction on operating on real numbers (whole numbers, integers, fractions and rational numbers, and decimals) — topics that were already part of the course.

\section{Implications}

The findings of this study suggest that preservice teachers might be completing their teacher education programs without appropriate preparation to teach early algebra content. The findings also show the challenges that teacher education programs have in preparing wellrounded elementary-school teachers that are knowledgeable and have strong understandings of content and concepts they are expected to teach after completing their respective teacher education programs. Elementary school teachers are expected to help young students of mathematics learn about properties and be able to use those properties when investigating and calculating with whole numbers and, more generally, rational numbers. However, if teachers 
themselves do not have well-formed knowledge of structural properties, then they cannot help young learners develop appropriate understandings of those properties.

\section{Reflecting on the Teaching Intervention}

This study was concerned with pre-service teachers' early algebra knowledge. Carraher and Schliemann (2007) described early algebra as encompassing "algebraic reasoning and algebra-related instruction among young learners - from approximately 6 to 12 years of age" (p. 670). This meant that, according to Carraher and Schliemann, early mathematics learning needed to emphasize the inherently algebraic character situated in the domain of arithmetic. According to Kanbir (2016) and Kanbir et al. (2018), exploiting the algebraic nature of early mathematics included giving special attention to properties of real numbers as part of early algebra instruction. This study explored what preservice teachers knew and understood about properties of real numbers - an important aspect of early algebra. This study was also concerned with preservice teachers' structure sense. In the context of early algebra, structure sense refers to one's capacity to apply properties of real numbers to manipulate mathematical expressions so that they be readily simplified, evaluated, or interpreted (Kanbir, 2016; Kanbir et al., 2018; Hoch \& Dreyfus, 2004; Novotna \& Hoch, 2008). This study investigated whether pre-service teachers could apply properties of real numbers to simplify, evaluate, and interpret mathematical expressions.

The goal of the teaching intervention was for MT1 PETs to gain relational understandings of properties of real numbers and of early algebra. Consequently, the intervention was expected to help MT1 PETs develop improved cognitive structures, and concept images, with respect to structure sense. Following Carraher and Schliemann's (2007) proposal that early algebra instruction should emphasize the inherent algebraic character of arithmetic, the 
intervention in this study sought to do so in the MT1 course by giving properties of real numbers an elevated focus during instruction on real number operations. After participating in the instructional intervention, the objective was to get MT1 PETs to the point where they could express confidently, using their own words, the early algebra concepts under consideration in this study. This objective was largely not achieved. Despite the constraints of implementing the instructional intervention, I expected to observe educationally significant gains between the preteaching and post-teaching knowledge and understandings of participating MT1 PETs.

Part of the challenge in this dissertation study was situating the MT1 intervention within one semester while using the pre-determined course schedule and ensuring all other course topics were also covered. The intervention involved emphasizing the role of properties, and usefulness of properties, in simplifying and evaluating arithmetic calculations. The intervention had two phases. The first phase included introducing the properties, defining them, and using them to compute with whole numbers. This phase covered four sessions of regular classroom time and spread over a period of two weeks. The second phase included the expanding the number system and using number properties when operating on integers, rational numbers, and real numbers, progressively and respectively. The second phase covered four sessions of classroom instruction spread out over a period of four weeks.

Numerical structure sense is not something that can be imposed on any learner. It must be developed gradually by helping learners build up fundamental ideas, such as basic arithmetic properties and the role of properties in arithmetic computations (Barnett \& Ding, 2019; Kanbir et al., 2018). Kanbir (2016) successfully helped the middle-school students in his study gain relational understandings of properties of real numbers and develop improved cognitive structures, and concept images, of numerical structure sense. The intervention in the Kanbir 
study comprised "a combination of two lesson sequences which altogether would cover 14 sessions of regular mathematics classroom time spread over a period of six weeks" (Kanbir, 2016, p. 91).

Another challenge in implementing the intervention involved situating the study in an undergraduate course that was constrained by time and the wide of range of topics that were required to be covered in one semester. College rules regarding deviations from the approved syllabus and course schedule created further constraints for implementing the intervention. If I were to complete this study again, I would petition to have more freedom over the course schedule and the intervention would be spread out over several sessions as needed. Kanbir (2016) and Kanbir et al. (2018) created model lessons and materials that can be used, or replicated, by any teacher to help learners develop relational understanding of properties and of numerical structure in school mathematics. I would employ those model lessons and materials if I were to complete another study on preservice teachers' numerical structure sense.

Kanbir (2016) "emphasized the need to take account of what was already 'in the minds' of learners during mathematics lessons" (p. 28). An effective intervention is one that builds from what participants already know. Preservice teachers in this study did not demonstrate wellformed concept images of the properties of real numbers. However, they did exhibit vague memories of prior knowledge regarding structural properties. Some the participating PETs knew that the operands in addition and multiplication sentences could be interchanged with affecting the result. Others expressed knowledge of the fact that ignoring the parentheses in addition or multiplication sentences did not affect the result of the sum or product, respectively. Participating PETs may have exhibited knowledge of these aforementioned ideas but hardly any of them connected those ideas to the commutative properties for addition/multiplication or the 
associative properties for addition/multiplication. When planning another investigation, I would take this into account; I would consider the existing cognitive structures that may exist in PETs' minds.

\section{Implications for Teaching}

With respect to tasks framed with properties of real numbers in mind, mathematics teachers need to "assist learners to recognize signs, give the signs appropriate meanings, and then do the mathematics demanded by what is signified in the tasks in which the signs are present" (Kanbir, 2016, p. 61). If prospective teachers themselves do not have opportunities to form welldeveloped knowledge of structural properties while completing their teacher preparation programs, then they cannot help young learners develop appropriate understandings of properties. Thus, the challenge for teacher education programs is to prepare well-rounded elementary-school teachers that are knowledgeable and have strong understandings of content and concepts they are expected to teach, including strong knowledge and understandings of properties of operations.

The emphasis in this study has been on the importance of structural properties and the need to prepare teachers with well-formed cognitive structures, and concept images, of structural properties. It should not be taken to mean that learning about order of operations is unimportant. I am just calling attention to rote learning of order of operations, which has been shown to be a hindrance on young learners' burgeoning understanding of structure in mathematics (Dupree, 2016; Kanbir, 2016. Kanbir et al., 2018; Lee, Licwinko, \& Taylor-Buckner, 2013). "With rigid memorization of the order of operations demanded by PEMDAS, students can not only get wrong answers for calculations, but also fail to learn important structural principles" (Kanbir, 2016, p. 43). The study of order of operations is important. Instead of rigid memorization 
demanded by PEMDAS, emphasizing the connections among arithmetic operations would provide students the opportunity "to understand PEMDAS with mathematical reasoning" (Lee at al., 2013, p. 74).

\section{Implications for Future Interventions}

In a future related intervention I will keep the observations of this study in mind. The intervention in this study did not produce educationally significant improvements with regards to MT1 PETs' knowledge of properties. In a future intervention, I may restrict the focus to whole numbers - introducing the properties, defining them, and using them to compute with whole numbers. The goal is to help PETs learn and understand the meanings and usefulness of properties when operating on real numbers, but PETs' limited understanding of decimals and fractional numbers dissuades them from fully appreciating the power of properties when operating on decimals and fractions. For example, in this study, when computing $(36 \times 5) \times 2$ some participating PETs recognized and used the associative property for multiplication, but the

same PETs did not do the same and rely on the associative property when computing $48 \times\left(\frac{1}{24} \times\right.$ 150). Desta (2019) highlighted the challenges that PETs have in "connecting different representations of rational number concepts and [PETs'] limitations with the language of fractions" (p. 203).

It is my desire to have a more comprehensive intervention that encompasses not only PETs' knowledge of number properties but also their knowledge of decimal and fractional numeration. Such an intervention would be part of an investigation that examines whether the MT1 course sufficiently helps PETs sharpen their knowledge of whole numbers, decimals, fractions and operations on these numbers. PETs' participation in a course such as MT1 should 
enable them to become aware of their misconceptions regarding fundamental mathematics, and to then help them amend those misconceptions (Clements \& Ellerton, 2009; Duarte, 2010). In accordance with NCTM's (2000) call to help learners identify and use properties, a course such as MT1 should assist PETs to recognize properties, to accurately define properties, and to use those properties when operating on whole numbers, decimals, and fractional numbers.

The aspiration is to have a comprehensive intervention that overhauls the MT1 course, but I may need to start with smaller targeted interventions that focus on assisting PETs to recognize properties, to define properties, and to use those properties to compute with whole numbers. For small targeted interventions on properties of whole numbers, I would allot ten sessions of classroom instruction spread out over a period of five weeks. The goal will be to have an instructional intervention that not only produces educationally significant gains but also results in long-term retention of what is learned. Clements and Ellerton's (2009) instructional intervention sequence of assessing $\rightarrow$ teaching $\rightarrow$ reflecting $\rightarrow$ revisiting could be useful in helping PETs learn and retain knowledge on properties of operations. Clements and Ellerton (2009) articulated the assessing $\rightarrow$ teaching $\rightarrow$ reflecting $\rightarrow$ revisiting intervention sequence in their " $5-\mathrm{R}$ Intervention Model," which contains five ordered intervention components-Realize, Review, Reflect, Revisit and Retain.

Each of the five components in the 5-R Intervention Model requires action on the part of the learner. In a prospective targeted intervention on number properties, participating PETs will first complete a pre-assessment before teaching occurs. After whole-class teaching, PETs will be asked to reflect on their pre-teaching assessment and individually write reflections about where they went wrong and why. Following the written reflections, PETs will complete a parallel postteaching assessment. The intervention process is expected to help participating PETs realize and 
confront what they do not know about properties of whole numbers; review and be guided toward appropriate conceptual understandings of properties during teaching; reflect by writing individual reflections; revisit their conceptual understanding of properties of whole numbers over some extended period of time. Lastly, participating PETs will complete an assessment to ascertain whether they acquired and retained their new/improved understandings of properties of whole numbers.

\section{Recommendations for Future Research}

This study investigated preservice elementary-school teachers' knowledge of the properties of real numbers. It was framed from the perspective that elementary-school teachers of mathematics should help young learners develop numerical structure sense and stronger conceptual understanding of early algebra for later success in algebra and higher mathematics. This study contributes to the literatures on structure sense, early algebra, and preservice teachers' readiness to teach early algebra concepts.

Future research in this area should improve on the design of this study. In this study, I attempted to deal with all major structural aspects of early algebra: commutative properties, associative properties, identity elements, inverse properties, and the distributive property. Closure properties and order of operations were part of classroom instruction but were not the subject of investigation in this dissertation study. Future research should include interventions that incorporate the model lessons and materials in Kanbir (2016) and Kanbir et al. (2018) studies and should take into account all major structural aspects including closure properties and order of operations.

A major objective for education research should be preparing "curriculum materials that would be easily usable by practicing teachers" (Kanbir, 2016, p. 90). Future researchers should 
create model lessons and materials on early algebra structure for use in an undergraduate mathematics course for preservice elementary teachers. Further, future researchers should plan an intervention study that follows preservice teachers from their university mathematics classrooms, where they are students, to classrooms in the field, where they are teachers. The goal for doing this is to not only investigate the pre- and post-intervention structural knowledge of prospective teachers, but also investigate the same group of prospective teachers teach lessons on the content covered in the intervention. It would be useful to see the effect participating in the intervention has on the students of prospective teachers after they have become credentialed teachers. Such a study would inform us whether classroom interventions can prepare wellknowledgeable elementary-school teachers that effect authentic learning in their own classrooms. 


\section{REFERENCES}

Anthony, G., \& Walshaw, M. (2002). Swaps and switches: Students' understandings of commutativity. In B. Barton, K. C. Irwin, M. Pfannkuch \& M. O. J. Thomas (Eds.), Proceedings of the 25 th annual conference of the Mathematics Education Research Group of Australasia (pp. 91-99). Auckland, New Zealand: Mathematics Education Research Group of Australasia.

Baek, J.-M. (2008). Developing algebraic thinking through explorations in multiplication. In C. E. Greenes \& R. Rubenstein (Eds.), Algebra and Algebraic Thinking in School Mathematics: Seventieth yearbook of the National Council of Teachers of Mathematics (NCTM; pp. 141-154). Reston, VA: NCTM.

Barrington, K. (2020, January 29). What is an articulation agreement and why should you care? Community College Review. https://www.communitycollegereview.com/blog/what-isan-articulation-agreement

Barnett, E., \& Ding, M. (2019). Teaching of the associative property: A natural classroom investigation. Investigations in Mathematics Learning, 11(2), 148-166. https://doi.org/10.1080/19477503.2018.1425592

Baroody, A. J. (2006). Why children have difficulties mastering the basic number combinations and how to help them. Teaching Children Mathematics, 13(1), 22-31.

Blanton, M. L., \& Kaput, J. J. (2005). Characterizing a classroom practice that promotes. Journal for Research in Mathematics Education, 36(5), 412-444. https://doi.org/10.2307/30034944 
Blanton, M. L., \& Kaput, J. J. (2011). Functional thinking as a route into algebra in the elementary grades. In J. Cai \& E. Knuth (Eds.), Early algebraization: A global dialogue from multiple perspectives (pp. 5-23). Heidelberg, Germany: Springer.

Blanton, M. L., Stephens, A., Knuth, E., Gardiner, A. M., Isler, I., \& Kim, J.-S. (2015). The development of children's algebraic thinking: The impact of a comprehensive early algebra intervention in third grade. Journal for Research in Mathematics Education, 46(5), 511-558. https://doi.org/10.5951/jresematheduc.46.1.0039

Booth, L. (1988). Children's difficulties in beginning algebra. In A. F. Coxford \& A. P. Shulte (Eds.), The ideas of algebra, K-12. 1988 Yearbook (pp. 20-32). Reston, VA: National Council of Teachers of Mathematics.

Cai, J., \& Knuth, E. (2011). Early algebraization: A global dialogue from multiple perspectives. Heidelberg, Germany: Springer.

Carpenter, T. P., Levi, L., Berman, P., \& Pligge, M. (2005). Developing algebraic reasoning in the elementary school. In T. A. Romberg, T. P. Carpenter, \& F. Dremock (Eds.), Understanding Mathematics and Science Matters (pp. 81-98). Mahwah, NJ: Lawrence Erlbaum.

Carraher, D. W., \& Schliemann, A. D. (2007). Early algebra and algebraic reasoning. In F. K. Lester (Ed.), Second handbook of research on mathematics teaching learning (pp. 669705). Charlotte, NC: Information Age Publishing.

Carraher, D. W., Schliemann, A. D., Brizuela, B. M., \& Earnest, D. (2006). Arithmetic and algebra in early mathematics education. Journal for Research in Mathematics Education, $32(2), 87-115$.

Chandler, D. (2007). Semiotics: The basics. New York, NY: Routledge. 
Clements, M. A., \& Ellerton, N. F. (2009). A model for improving prospective mathematics teachers' algebra content knowledge. Brunei International Journal of Science and Mathematics Education, 1(1), 65-83.

Coe, R. (2002, September). It's the effect size, stupid: What effect size is and why it is important. Presented at the Annual Conference of the British Educational Research Association, University of Exeter, England. Retrieved from www.leeds.ac.uk/educol/documents/00002182.htm

Cohen, J. (1988). Statistical power analysis for the behavioral sciences (2nd ed.). Hillsdale, NJ: Lawrence Erlbaum Associates.

de Saussure, F. (1959). Course in general linguistics. New York, NY: The Philosophical Library.

Del Campo, G., \& Clements, M. A. (1987). A manual for the professional development of teachers of beginning mathematicians. Melbourne, Australia: Association of Independent Schools of Victoria.

Derrick, B., Toher, D., \& White, P. (2016). Why Welchs test is Type I error robust. The Quantitative Methods for Psychology, 12 (1), 30-38. https://doi.org/10.20982/tqmp.12.1.p030

Desta, D. D. (2019). Preservice teachers making sense of fraction concepts and operations in a mathematics education content course (Ph.D. Dissertation). Illinois State University, Normal, IL.

Ding, M. (2016). Developing preservice elementary teachers' specialized content knowledge: The case of associative property. International Journal of STEM Education, 3(9), 1-19. https://doi.org/10.1186/s40594-016-0041-4 
Ding, M., Li, X., \& Capraro, M. M. (2013). Preservice elementary teachers' knowledge for teaching the associative property of multiplication: A preliminary analysis. The Journal of Mathematical Behavior, 32(1), 36-52. https://doi.org/10.1016/j.jmathb.2012.09.002

Duarte, J. (2010). The effects of an undergraduate algebra course on prospective middle-school teachers' understanding of functions, especially quadratic functions (Ph.D. Dissertation). Illinois State University, Normal, IL.

Dupree, K. M. (2016). Questioning the order of operations. Mathematics Teaching in the Middle School, 22(3), 152-59.

Eco, U. (1976). A theory of semiotics. Bloomington, IN: Indiana University Press.

Ellerton, N. F., \& Clements, M. A. (1991). Mathematics in language: A review of language factors in mathematics learning. Geelong, Australia: Deakin University.

Ellerton, N. F., \& Clements, M. A. (2011). Prospective middle-school mathematics teachers' knowledge of equations and inequalities. In J. Cai \& E. Knuth (Eds.), Early algebraization: A global dialogue from multiple perspectives (pp. 379-409). Heidelberg, Germany: Springer.

Fan, X. (2001). Statistical significance and effect size in education research: Two sides of a coin. The Journal of Educational Research, 94(5), 275-282. https://doi.org/10.1080/00220670109598763

Fauskanger, J., \& Bjuland, R. (2019). Learning ambitious teaching of multiplicative properties through a cycle of enactment and investigation. Mathematics Teacher Education and Development, 2019(1), 125-144. 
Fennema, E., \& Franke, M. L. (1992). Teachers' knowledge and its impact. In D. Grouws (Ed.), Handbook of research on mathematics teaching and learning (pp. 147-164). New York, NY: Macmillan.

Gagné, R. M. (1985). The conditions of learning (4th ed.). New York, NY: Holt, Rinehart \& Winston.

Gagné, R. M., \& Merrill, M. D. (1990). Integrative goals for instructional design. Educational Technology Research and Development, 38(1), 23-30. https://doi.org/10.1007/BF02298245

Gagné, R. M., \& White, R. T. (1978). Memory structures and learning outcomes. Review of Education Research, 48(2), 87-222. https://doi.org/10.3102/00346543048002187

Glesne, C. (1999). Becoming qualitative researchers: An introduction. Don Mills, Canada: Longman.

Hart, K. M. (1981). Children's understanding of mathematics: 11-16. London, United Kingdom: Murray.

Herbart, J. F. (1898). Letters and lectures on education. (H. M. Felkin \& E. Felkin, Trans.). London, United Kingdom: Swan Sonnenschein \& Co.

Hoch, M., \& Dreyfus, T. (2004). Structure sense in high school algebra: The effect of brackets. In M. J. Hoines \& A. B. Fuglestad (Eds.), Proceedings of the 28th Conference of the International Group for the Psychology of Mathematics Education (Vol. 3, pp. 49-56). Bergen, Norway: International Group for the Psychology of Mathematics Education. Howe, R. (2005). Comments on NAEP algebra problems. Retrieved from https://www.brookings.edu/wp-content/uploads/2012/04/Howe_Presentation.pdf 
Hurst, C. (2017). Children have the capacity to think multiplicatively, as long as .... European Journal of STEM Education, 2(3), 1-14. https://doi.org/10.20897/ejsteme/78169

Hurst, C., \& Huntley, R. (2017). Explicitly connecting mathematical ideas: How well is it done? In A. Downton, S. Livy, \& J. Hall (Eds.), Proceedings of the $40^{\text {th }}$ Annual Conference of the Mathematics Education Research Group of Australasia (pp. 325-332). Melbourne, Australia: Mathematics Education Research Group of Australasia.

Jeon, K. (2012). Reflecting on PEMDAS. Teaching Children Mathematics, 18(6), 370-377. https://doi.org/10.5951/teacchilmath.18.6.0370

Kaminski, E. (2002). Promoting mathematical understanding: Number sense in action. Mathematics Education Research Journal, 14(2), 133-149.

Kanbir, S, Clements, M. A., \& Ellerton, N. F. (2018). Using design research and history to tackle a fundamental problem with school algebra. New York, NY: Springer.

Kanbir, S. (2016). An intervention study aimed at enhancing seventh-grade students' development of the concept of a variable (Ph.D. Dissertation). Illinois State University, Normal, IL.

Kaput, J. J. (2008). What is algebra? What is algebraic reasoning? In J. J. Kaput, D. W. Carraher, \& M. L. Blanton (Eds.), Algebra in the early grades (pp. 5-17). New York, NY: Lawrence Erlbaum Associates.

Kaput, J. J., Carraher, D. W., \& Blanton, M. L. (Eds.). (2008). Algebra in the early grades. New York, NY: Lawrence Erlbaum Associates.

Kieran, C. (1992). The learning and teaching of school algebra. In D. Grouws (Ed.), Handbook of research on mathematics teaching and learning (pp. 309-419). New York, NY: Macmillan. 
Kieran, C. (2007). Learning and teaching algebra at the middle-school through college levels: Building meaning for symbols and their manipulation. In F. K. Lester (Ed.), Second handbook of research on mathematics teaching and learning (pp. 707-762). Charlotte, NC: Information Age Publishing.

Kinzer, C. J., \& Stanford, T. (2013). The distributive property: The core of multiplication. Teaching Children Mathematics, 20(5), 302-309.

Kose, N. Y., \& Kiziltoprak, A. (2020). Development of secondary school students' relational thinking skills with a teaching experiment. Eurasian Journal of Educational Research, 85(2020), 135-168.

Larsen, S. (2010). Struggling to disentangle the associative and commutative properties. For the Learning of Mathematics, 30(1), 37-42.

Lee, J. K., Licwinko, S., \& Taylor-Buckner, N. (2013). Exploring mathematical reasoning of the order of operations: Rearranging the procedural component PEMDAS. Journal of Mathematics Education at Teachers College, 4(2), 73-78. https://doi.org/10.7916/jmetc.v4i2.633

Lins, R., \& Kaput, J. J. (2004). The early development of algebraic reasoning: The current state of the field. In K. Stacey, H. Chick, \& M. Kendal (Eds.), The future of the teaching and learning of algebra The 12th ICMI Study (pp. 45-70). Melbourne, Australia: Springer, Dordrecht.

Ma, L. (1999). Knowing and teaching elementary mathematics: Teachers' understanding of fundamental mathematics in China and the United States. Mahwah, NJ: Erlbaum. 
Mason, J., Stephens, M., \& Watson, A. (2009). Appreciating mathematical structure for all. Mathematics Education Research Journal, 21(2), 10-32. https://doi.org/10.1007/BF03217543

Monandi, O. O. (2018). Pre-service elementary teachers' knowledge of number properties and patterns in the context of early algebra. Issues in the Undergraduate Mathematics of School Teachers (IUMPST): The Journal, 1. Retrieved from http://www.k12prep.math.ttu.edu/journal/1.contentknowledge/monandi01/article.pdf

Monandi, O. O. (2019). Connecting concepts in group theory to properties of real numbers in a mathematics course for preservice teachers. Unpublished manuscript, Illinois State University.

National Council of Teachers of Mathematics. (1989). Curriculum and evaluation standards for school mathematics. Reston, VA: Author.

National Council of Teachers of Mathematics. (2000). Principles and standards for school mathematics. Reston, VA: Author.

National Council of Teachers of Mathematics. (2006). Curriculum focal points for Prekindergarten through grade 8 mathematics: A quest for coherence. Reston, VA: Author.

National Governors Association for Best Practices, \& Council of Chief State School Officers. (2010). Common Core State Standards for Mathematics. Washington, DC: Authors.

Novotná, J., \& Hoch, M. (2008). How structure sense for algebraic expressions or equations is related to structure sense for abstract algebra. Mathematics Education Research Journal, 20(2), 93-104. https://doi.org/10.1007/BF03217479 
Peirce, C. S. (1992). The essential Peirce: Selected philosophical writings (Vol. 1). Bloomington, IN: Indiana University Press.

Peirce, C. S. (1998). The essential Peirce (Vol. 2). Bloomington, IN: Indiana University Press.

Powell, S., Ding, Y., Wang, Q., Craven, J., \& Chen E. (2019). Exploring strategy use for multiplication problem solving in college students. International Journal of Research in Education and Science (IJRES), 5(1), 374-387.

Presmeg, N. (2014). Mathematics education research embracing arts and sciences. In M. Fried \& T. Dreyfus (Eds.), Mathematics and mathematics education: Searching for common ground (pp. 361-378). New York, NY: Springer.

Presmeg, N., Radford, L., Roth, W.-M., \& Kadunz, G. (2016). Semiotics in theory and practice in mathematics education. In G. Kaiser (Ed.), Semiotics in Mathematics Education. ICME-13 Topical Surveys. Hamburg, Germany: Springer, Cham.

Radford, L. (2006). Algebraic thinking and the generalization of patterns: A semiotic perspective. In S. Alatorre, J. J. Cortina, M. Saiz, \& A. Mendez (Eds.), Proceedings of the 28th Annual Meeting of the North American Chapter of the International Group for the Psychology of Mathematics Education (Vol. 1, pp. 2-21). Merida, Mexico: International Group for the Psychology of Mathematics Education.

Radford, L. (2011). Grade 2 students' non-symbolic algebraic thinking. In J. Cai, \& E. Knuth (Eds.), Early algebraization: A global dialogue from multiple perspectives (pp. 303-322). Heidelberg, Germany: Springer.

Satterthwaite, F. E. (1946). An approximate distribution of estimates of variance components. Biometrics Bulletin, 2, 110-114. https://doi.org/10.2307/3002019 
Schifter, D., Monk, S., Russell, S. J., \& Bastable, V. (2008). Early algebra: What does understanding laws of arithmetic mean in the elementary grades? In J. J. Kaput, D. W. Carraher, \& M. L. Blanton (Eds.), Algebra in the early grades (pp. 413-447). New York, NY: Lawrence Erlbaum Associates.

Stacey, K., \& MacGregor, M. (2000). Learning the algebraic method of solving problems. Journal of Mathematical Behavior, 18(2), 149-167. https://doi.org/10.1016/S07323123(99)00026-7

Stephens, A. C., Ellis, A. B., Blanton, M., \& Brizuela, B. M. (2017). Algebraic thinking in the elementary and middle grades. In J. Cai (Ed.), Compendium for research in mathematics education (pp. 386-420). Reston, VA: The National Council of Teachers of Mathematics.

Sullivan, G. M., \& Feinn, R. (2012). Using effect size—or why the $p$ value is not enough. Journal of Graduate Medical Education, 4(3), 279-282. https://doi.org/10.4300/JGMED-12-00156.1

Swafford, J. O., \& Langrall, C. W. (2000). Grade 6 students' preinstructional use of equations to describe and represent problem situations. Journal for Research in Mathematics Education, 31(1), 89-112. https://doi.org/10.2307/749821

Tall, D., \& Vinner, S. (1981). Concept image and concept definition in mathematics with particular reference to limits and continuity. Educational Studies in Mathematics, 12(2), 151-169. https://doi.org/10.1007/BF00305619

Thanheiser, E., Philipp, R. A., Fasteen, J., Strand, K., \& Mills, B. (2013). Preservice teacher interviews: A tool for motivating mathematics learning. Mathematics Teacher Educator, 1(2), 137-147. https://doi.org/10.5951/mathteaceduc.1.2.0137 
Trent, M. W. (2006). Understanding the properties of arithmetic: A prerequisite for success in algebra. Mathematics Teaching in the Middle School, 12(1), 22-25.

Usiskin, A. (1988). Conceptions of school algebra and uses of variables. In A. P. Schulte (Ed.), The ideas of algebra, K-12, 1988 Yearbook (pp. 8-19). Reston, VA: National Council of Teachers of Mathematics.

Vaiyavutjamai, P. (2003). Dilemmas associated with quantitative analyses of effects of traditional mathematics teaching. In H. S. Dhindsa, S. B. Lee, P. Achleitner, \& M. A. Clements (Eds.), Studies in science, mathematics and technical education (pp. 259-268). Gadong, Brunei Darussalam: Universiti Brunei Darussalam.

Vinner, S., \& Dreyfus, T. (1989). Images and definitions for the concept of function. Journal for Research in Mathematics Education, 20(4), 356-366.

Vinner, S., \& Hershkowitz, R. (1980). Concept images and some common cognitive paths in the development of some simple geometric concepts. In R. Karplus (Ed.), Proceedings of the Fourth International Conference for the Psychology of Mathematics Education (pp. 177184). Berkeley, CA: International Group for the Psychology of Mathematics Education.

Wang, X. (2015). The literature review of algebra learning: Focusing on the contributions to students' difficulties. Creative Education, 6, 144-153. https://doi.org/10.4236/ce.2015.62013

Warren, E. \& English, L. (2000). Primary school children's knowledge of arithmetic structure. In J. Bana and A. Chapman (Eds.), Proceedings of $23^{\text {rd }}$ annual conference of the Mathematics Education Research Group of Australasia (pp. 624-631). Fremantle, Australia: Mathematics Education Research Group of Australasia. 
Welch, B. L. (1947). The generalization of "student's" problem when several different population variances are involved. Biometrika, 34, 28-35. https://doi.org/10.2307/2332510

Welder, R. M. (2012). Improving algebra preparation: Implications from research on student misconceptions and difficulties. School Science and Mathematics, 112(4), 255-264. https://doi.org/10.1111/j.1949-8594.2012.00136.x

You, Z. (2006). Preservice teachers' knowledge of linear functions within multiple representation modes (Ph.D. Dissertation). Texas A \& M University. 


\section{APPENDIX A: NUMBER PROPERTIES IN THE COMMON CORE STANDARDS}

The following are samples of CCSSM content statements containing language highlighting the pertinence of properties of real numbers in elementary and middle-school curricula.

\section{Grade 1}

\section{CCSS.MATH.CONTENT.1.OA.B.3}

Apply properties of operations as strategies to add and subtract. Examples: If $8+3=11$ is known, then $3+8=11$ is also known (commutative property of addition). To add $2+6+4$, the second two numbers can be added to make a ten, so $2+6+4=2+10=12$ (associative property of addition) (NGA \& CCSSO, 2010, p. 15).

\section{Grade 2}

\section{CCSS.MATH.CONTENT.2.NBT.B.5}

Fluently add and subtract within 100 using strategies based on place value, properties of operations, and/or the relationship between addition and subtraction. (NGA \& CCSSO, 2010, p. 19)

\section{CCSS.MATH.CONTENT.2.NBT.B.6}

Add up to four two-digit numbers using strategies based on place value and properties of operations. (NGA \& CCSSO, 2010, p. 19)

\section{CCSS.MATH.CONTENT.2.NBT.B.9}

Explain why addition and subtraction strategies work, using place value and the properties of operations. (NGA \& CCSSO, 2010, p. 19) 


\section{Grade 3}

\section{CCSS.MATH.CONTENT.3.OA.B.5}

Apply properties of operations as strategies to multiply and divide. Examples: If $6 \times 4=24$ is known, then $4 \times 6=24$ is also known (commutative property of multiplication). $3 \times 5 \times 2$ can be found by $3 \times 5=15$, then $15 \times 2=30$, or by $5 \times 2=10$, then $3 \times 10=30$ (associative property of multiplication).

Knowing that $8 \times 5=40$ and $8 \times 2=16$, one can find $8 \times 7$ as $8 \times(5+2)=(8 \times 5)+(8 \times 2)$ $=40+16=56$ (distributive property). (NGA \& CCSSO, 2010, p. 23)

\section{CCSS.MATH.CONTENT.3.NBT.A.2}

Fluently add and subtract within 1000 using strategies and algorithms based on place value, properties of operations, and/or the relationship between addition and subtraction. (NGA \& CCSSO, 2010, p. 24)

\section{Grade 4}

\section{CCSS.MATH.CONTENT.4.OA.A.1}

Interpret a multiplication equation as a comparison, e.g., interpret $35=5 \times 7$ as a statement that 35 is 5 times as many as 7 and 7 times as many as 5 . Represent verbal statements of multiplicative comparisons as multiplication equations. (NGA \& CCSSO, 2010, p. 29)

\section{CCSS.MATH.CONTENT.4.OA.A.2}

Multiply or divide to solve word problems involving multiplicative comparison, e.g., by using drawings and equations with a symbol for the unknown number to represent the problem, distinguishing multiplicative comparison from additive comparison. (NGA \& CCSSO, 2010, p. 29) 


\section{Grade 5}

\section{CCSS.MATH.CONTENT.5.OA.A.1}

Use parentheses, brackets, or braces in numerical expressions, and evaluate expressions with these symbols. (NGA \& CCSSO, 2010, p. 35)

\section{CCSS.MATH.CONTENT.5.OA.A.2}

Write simple expressions that record calculations with numbers and interpret numerical expressions without evaluating them. For example, express the calculation "add 8 and 7, then multiply by $2^{\prime \prime}$ as $2 \times(8+7)$. Recognize that $3 \times(18932+921)$ is three times as large as 18932 +921, without having to calculate the indicated sum or product. (NGA \& CCSSO, 2010, p. 35)

\section{Grade 6}

\section{CCSS.MATH.CONTENT.6.EE.A.1}

Write and evaluate numerical expressions involving whole-number exponents. (NGA \& CCSSO, 2010, p. 43)

\section{CCSS.MATH.CONTENT.6.EE.A.2}

Write, read, and evaluate expressions in which letters stand for numbers. (NGA \& CCSSO, 2010, p. 43

\section{CCSS.MATH.CONTENT.6.EE.A.3}

Apply the properties of operations to generate equivalent expressions. For example, apply the distributive property to the expression $3(2+x)$ to produce the equivalent expression $6+3 x$; apply the distributive property to the expression $24 x+18 y$ to produce the equivalent expression $6(4 x+3 y) ;$ apply properties of operations to $y+y+y$ to produce the equivalent expression $3 y$. (NGA \& CCSSO, 2010, p. 44) 


\section{Grade 7}

\section{CCSS.MATH.CONTENT.7.EE.A.1}

Apply properties of operations as strategies to add, subtract, factor, and expand linear

expressions with rational coefficients. (NGA \& CCSSO, 2010, p. 49)

CCSS.MATH.CONTENT.7.EE.A.2

Understand that rewriting an expression in different forms in a problem context can shed light on the problem and how the quantities in it are related. For example, $a+0.05 a=1.05 a$ means that "increase by 5\%" is the same as "multiply by 1.05." (NGA \& CCSSO, 2010, p. 49) 


\section{APPENDIX B: PROTOCOLS FOR INTERVIEWS (TWO PARALLEL VERSIONS)}

\section{(MT1 Pre-Teaching, MT2 and MT3 Interview Protocol)}

1. The interviews are audio-recorded.

2. The interviewer has the following:

a) A sheet of paper with $482+(18+300)$ on it. [See (2) below.]

b) A sheet of paper with value of $4 \times(1 / 4 \times 128)$ on it. [See (3) below.]

c) A sheet of paper with $315 \times 0.7+315 \times 0.3$ on it. [See (4) below.]

d) A sheet of paper with $5.25 x=70 \times 5.25+30 \times 5.25$ on it. [See (5) below.]

e) A sheet of paper with $92 \times 5$ on it. [See (6) below.]

f) A sheet of paper with $13+y=14+13$ on it. [See (7) below.]

g) A sheet of paper with $5 y=4 \times 5$ on it. [See (8) below.]

h) A sheet of paper with the terms additive identity and multiplicative identity written on it. [See (9) below.]

i) A sheet of paper with the terms additive inverse and multiplicative inverse written on it. [See (10) below.]

1. "I am going to say two words and, as soon as I say them, I want you to say something, or draw something, or do something — do the first thing that comes into your head after I say the words. The words are ... "distributive property." Here are the words again: "distributive property."

2. Without using a calculator, find the value of $482+(18+300)$.

[Once an answer is given ask for the explanation of where that answer came from.]

[After the student has given explanation, ask the student to think of a different way of finding solution.] 
3. Without using a calculator, find the value of $4 \times(1 / 4 \times 128)$.

[Once an answer is given ask for the explanation of where that answer came from.] [After the student has given explanation, ask the student to think of a different way of finding solution.]

4. Without using a calculator, what is the value of $315 \times 0.7+315 \times 0.3$ ?

[When the student gives an answer, ask her or him to write down how she or he obtained that answer. Also, ask the student to explain what she or he thought, in words. Then ask the student to think of another way of evaluating the expression]

5. Give the student a piece of paper with $5.25 x=70 \times 5.25+30 \times 5.25$ on it, then ask her or him to say which values of $x$ would make the equation true.

[When the student gives an answer, ask her or him to write down how she or he obtained that answer. Also, ask the student to explain what she or he thought, in words. Then ask the student to think of another way of finding solution.]

6. Give the student a piece of paper with $92 \times 5$ on it, then ask her or him to compute $92 \times 5$. [When the student gives an answer, ask her or him to explain how she or he obtained that answer. Also, ask the student to explain why his or her strategy for obtaining the answer works. Challenge the student to explain whether she or he is applying a mathematical property.] 
7. Give the student a piece of paper with $13+y=14+13$ on it, then ask her or him to say which values of $y$ would make the equation true.

[Once an answer is given ask for the explanation of where that answer came from.] [After the student has given explanation, ask the student to think of a different way of finding solution.]

8. Give the student a piece of paper with $5 y=4 \times 5$ on it, then ask her or him to say which values of $y$ would make the equation true.

[Once an answer is given ask for the explanation of where that answer came from.] [After the student has given explanation, ask the student to think of a different way of finding solution.]

9. "I am going to show you a sheet of paper with some words on it." [Show the pupil a piece of paper with the terms ADDITIVE IDENTITY and MULTIPLICATIVE IDENTITY.] "What do these terms mean?" [After the pupil gives his or her answer, ask him or her whether he or she had heard of these terms before. If the pupil responded with "I don't know" to the initial question, ask him or her to say what he or she thinks the terms mean.]

10. I am going to show you a sheet of paper with some words on it." [Show the pupil a piece of paper with the terms ADDITIVE INVERSE and MULTIPLICATIVE INVERSE.]

"What do these terms mean?"

[After the pupil gives his or her answer, ask him or her whether he or she had heard of these terms before. If the pupil responded with "I don't know" to the initial question, ask him or her to say what he or she thinks the terms mean.] 


\section{PROTOCOL FOR INTERVIEWS (TWO PARALLEL VERSIONS) \\ (MT1 POST-Teaching Interview Protocol)}

1. The interviews are audio-recorded.

2. The interviewer has the following:

a) A sheet of paper with $940+(60+403)$ on it. [See (2) below.]

b) A sheet of paper with value of $64 \times\left(\frac{1}{32} \times 120\right)$ on it. [See (3) below.]

A sheet of paper with value of $(72 \times 5) \times 2$ on it. [See (3) below.]

c) A sheet of paper with $7 \times 97+7 \times 3$ on it. [See (4) below.]

d) A sheet of paper with $5.25 x=70 \times 5.25+30 \times 5.25$ on it. [See (5) below.]

e) A sheet of paper with $84 \times 5$ on it. [See (6) below.]

f) A sheet of paper with $14+5=5+y$ on it. [See (7) below.]

g) A sheet of paper with $4 \times 112=112 y$ on it. [See (8) below.]

h) A sheet of paper with the terms additive identity and multiplicative identity written on it. [See (9) below.]

i) A sheet of paper with the terms additive inverse and multiplicative inverse written on it. [See (10) below.]

1. "I am going to say two words and, as soon as I say them, I want you to say something, or draw something, or do something — do the first thing that comes into your head after I say the words. The words are ... "distributive property." Here are the words again:

\section{“distributive property."}

2. Without using a calculator, find the value of $940+(60+403)$.

[Once an answer is given ask for the explanation of where that answer came from.] 
[After the student has given explanation, ask the student to think of a different way of finding solution.]

3. Without using a calculator, find the value of $64 \times\left(\frac{1}{32} \times 120\right)$. [Once an answer is given ask for the explanation of where that answer came from.] [After the student has given explanation, ask the student to think of a different way of finding solution.]

Without using a calculator, find the value of $(72 \times 5) \times 2$.

[Once an answer is given ask for the explanation of where that answer came from.]

[After the student has given explanation, ask the student to think of a different way of finding solution.]

4. Without using a calculator, what is the value of $7 \times 97+7 \times 3$ ?

[When the student gives an answer, ask her or him to write down how she or he obtained that answer. Also, ask the student to explain what she or he thought, in words. Then ask the student to think of another way of evaluating the expression]

5. Give the student a piece of paper with $5.25 x=70 \times 5.25+30 \times 5.25$ on it, then ask her or him to say which values of $x$ would make the equation true.

[When the student gives an answer, ask her or him to write down how she or he obtained that answer. Also, ask the student to explain what she or he thought, in words. Then ask the student to think of another way of finding solution.]

6. Give the student a piece of paper with $84 \times 5$ on it, then ask her or him to compute $92 \times 5$. [When the student gives an answer, ask her or him to explain how she or he obtained that answer. Also, ask the student to explain why his or her strategy for obtaining the answer works. Challenge the student to explain whether she or he is applying a mathematical property.] 
7. Give the student a piece of paper with $14+5=5+y$ on it, then ask her or him to say which values of $y$ would make the equation true.[Once an answer is given ask for the explanation of where that answer came from.] [After the student has given explanation, ask the student to think of a different way of finding solution.]

8. Give the student a piece of paper with $4 \times 112=112 y$ on it, then ask her or him to say which values of $y$ would make the equation true.

[Once an answer is given ask for the explanation of where that answer came from.] [After the student has given explanation, ask the student to think of a different way of finding solution.]

9. "I am going to show you a sheet of paper with some words on it." [Show the pupil a piece of paper with the terms ADDITIVE IDENTITY and MULTIPLICATIVE IDENTITY.] "What do these terms mean?" [After the pupil gives his or her answer, ask him or her whether he or she had heard of these terms before. If the pupil responded with "I don't know" to the initial question, ask him or her to say what he or she thinks the terms mean.]

10. I am going to show you a sheet of paper with some words on it." [Show the pupil a piece of paper with the terms ADDITIVE INVERSE and MULTIPLICATIVE INVERSE.] "What do these terms mean?" [After the pupil gives his or her answer, ask him or her whether he or she had heard of these terms before. If the pupil responded with "I don't know" to the initial question, ask him or her to say what he or she thinks the terms mean.] 
APPENDIX C: PAPER-AND-PENCIL ASSESSMENTS (TWO PARALLEL VERSIONS)

\section{Assessment I (MT1 Pre-Teaching, MT2 and MT3 Version)}

1. A really important property for numbers and for algebra is called the commutative property for multiplication. Describe this property.

2. Suppose you were asked to calculate the value of $940+(60+427)$ in your head. How would you do it, and which property would you be using?

Can you think of a different way of evaluating $940+(60+427) ?$ Explain.

3. If $13 \times y=9 \times 13$, what must $y$ equal?

Which property allows you to quickly determine the value of $y$ ? 
4. What must $x$ equal if $3 x=21 \times 3+4 \times 3$ ? Explain how you got your answer.

Is there another way of finding the value of $x$ if $3 x=21 \times 3+4 \times 3$ ? Explain.

5. Without using a calculator find the value of $(72 \times 5) \times 2$ and explain how you got your answer.

Can you think of a different way of evaluating $(72 \times 5) \times 2$ ? Explain.

6. What must $x$ equal if $12-(8-4)=(12-x)-4$ ? Explain how you got your answer. 
7. Without using a calculator find the value of $0.6 \times 250+250 \times 0.4$ and explain how you got your answer.

Can you think of a different way of evaluating $0.6 \times 250+250 \times 0.4$ ? Explain.

8. If $20 \times(10+5)=(20 \times 10)+(20 \times y)$, what must $y$ equal?

Explain how you got your answer. 
9. If $16+26=x+16$, what must $x$ equal?

Which property allows you to quickly determine the value of $x$ ?

10. What would be a quick method of finding the value of $\left(120 \times \frac{1}{32}\right) \times 64$, without using a calculator? What is the property which allows you to use that quick method?

11. What would be a quick method of finding the value of $\frac{1}{5} \times 96+\frac{1}{5} \times 4$ without using a calculator? What is the property which allows you to use that quick method?

12. Another important property for numbers and for algebra is called the associative property for addition. Describe this property. 
13. What must be the value of $y$ if $(10-y)-(10-5)=0$ ?

Explain your answer.

14. Terry was solving the problem below. Each line shows the property used to move from one step to the next. Write the appropriate expression for each step.

$$
45+(56+-45)
$$

a.

b.

c.

d.

e.
Commutative property for addition

Associative property for addition

Additive Inverse Property

Additive Identity Property

15. Jackie was solving the problem below and completed it as follows. Provide the appropriate property that Jackie applied to move from one step to the next step.

$$
3 \times 10 \times \frac{1}{3} \times 1.5
$$
a. $3 \times\left(10 \times \frac{1}{3}\right) \times 1.5$
b. $3 \times\left(\frac{1}{3} \times 10\right) \times 1.5$
c. $\left(3 \times \frac{1}{3}\right) \times 10 \times 1.5$
d. $\quad 1 \times 10 \times 1.5$
e. $\quad 10 \times 1.5$
f. 15 


\section{PAPER-AND-PENCIL ASSESSMENTS (TWO PARALLEL VERSIONS) Assessment II (MT1 Post-Teaching Version)}

1. A really important property for numbers and for algebra is called the commutative property for multiplication. Describe this property.

2. Suppose you were asked to calculate the value of $920+(80+533)$ in your head. How would you do it, and which property would you be using?

Can you think of a different way of evaluating $920+(80+533)$ ? Explain.

3. If $15 \times y=5 \times 15$, what must $y$ equal?

Which property allows you to quickly determine the value of $y$ ? 
4. What must $x$ equal if $3 x=21 \times 3+4 \times 3$ ? Explain how you got your answer.

Is there another way of finding the value of $x$ if $3 x=21 \times 3+4 \times 3$ ? Explain.

5. Without using a calculator find the value of $(36 \times 5) \times 2$ and explain how you got your answer.

Can you think of a different way of evaluating $(36 \times 5) \times 2$ ? Explain.

6. What must $x$ equal if $12-(8-4)=(12-x)-4$ ? Explain how you got your answer. 
7. Without using a calculator find the value of $0.8 \times 315+315 \times 0.2$ and explain how you got your answer.

Can you think of a different way of evaluating $0.8 \times 315+315 \times 0.2$ ? Explain.

8. If $50 \times(10+5)=(50 \times 10)+(50 \times y)$, what must $y$ equal?

Explain how you got your answer.

9. If $36+18=x+36$, what must $x$ equal?

Which property allows you to quickly determine the value of $x$ ? 
10. What would be a quick method of finding the value of $48 \times\left(\frac{1}{24} \times 150\right)$, without using a calculator? What is the property which allows you to use that quick method?

11. What would be a quick method of finding the value of $\frac{1}{5} \times 96+\frac{1}{5} \times 4$ without using a calculator? What is the property which allows you to use that quick method?

12. Another important property for numbers and for algebra is called the associative property for addition. Describe this property.

13. What must be the value of $y$ if $(15-y)-(15-5)=0$ ?

Explain your answer. 
14. Terry was solving the problem below. Each line shows the property used to move from one step to the next. Write the appropriate expression for each step.

$$
75+(86+-75)
$$

a.

b.

c.

d.

e.
Commutative property for addition

Associative property for addition

Additive Inverse Property

Additive Identity Property

15. Jackie was solving the problem below and completed it as follows. Provide the appropriate property that Jackie applied to move from one step to the next step.

$$
12 \times 100 \times \frac{1}{12} \times 1.5
$$
a. $12 \times\left(100 \times \frac{1}{12}\right) \times 1.5$
b. $12 \times\left(\frac{1}{12} \times 100\right) \times 1.5$
c. $\left(12 \times \frac{1}{12}\right) \times 100 \times 1.5$
d. $\quad 1 \times 100 \times 1.5$
e. $\quad 100 \times 1.5$
f. $\quad 150$ 
APPENDIX D: CHARTS FOR PROPERTIES OF WHOLE-NUMBER OPERATIONS

Properties of Whole Number Addition - Let $a, b$, and $c$ be whole numbers.

\begin{tabular}{|l|l|l|l|}
\hline \multicolumn{1}{|c|}{ Property } & Property Rule & Property Summarized & Example \\
\hline Commutative & & & \\
\hline Identity & & & \\
\hline Associative & & & \\
\hline Closure & & & \\
\hline
\end{tabular}

\section{EXAMPLE OF APPROPRIATELY COMPLETED CHART}

Properties of Whole Number Addition - Let $a, b$, and $c$ be whole numbers.

\begin{tabular}{|l|l|l|l|}
\hline Property & Property Rule & Property Summarized & Example \\
\hline Commutative & $a+b=b+a$ & $\begin{array}{l}\text { The order that I add terms } \\
\text { doesn't change the sum }\end{array}$ & $\begin{array}{l}7+5=12 \\
5+7=12\end{array}$ \\
\hline Identity & $\begin{array}{l}a+0=a \\
0+a=a\end{array}$ & $\begin{array}{l}\text { Adding zero doesn't } \\
\text { change my sum }\end{array}$ & $\begin{array}{c}0+17,842=17,482 \\
17,842+0=17,842\end{array}$ \\
\hline Associative & $(a+b)+c=a+(b+c)$ & $\begin{array}{l}\text { I can regroup terms (thereby } \\
\text { changing the order) without } \\
\text { changing my sum }\end{array}$ & $\begin{array}{l}(5+4)+3=9+3=12 \\
5+(4+3)=5+7=12\end{array}$ \\
\hline Closure & $a+b=c$ & $\begin{array}{l}\text { The sum of any two whole } \\
\text { is still } a \text { whole \# }\end{array}$ & $\begin{array}{l}57,872+837,294 \\
\text { numbers is a whole number. }\end{array}$ \\
$\begin{array}{l}\text { whaterer it is, it will be } \\
\text { a whole number. }\end{array}$ \\
\hline
\end{tabular}


Which Properties of Whole Number Addition Hold for Whole Number Subtraction?

Let $a, b$, and $c$ be whole numbers.

\begin{tabular}{|l|l|l|l|}
\hline Property Name & Property Rule/Summary & Example & $\begin{array}{c}\text { Does it hold for } \\
\text { Subtraction? }\end{array}$ \\
\hline Commutative & & & \\
\hline Identity & & & \\
\hline Associative & & & \\
\hline Closure & & & \\
\hline
\end{tabular}

EXAMPLE OF APPROPRIATELY COMPLETED CHART

Which Properties of Whole Number Addition Hold for Whole Number Subtraction? Let $a, b$, and $c$ be whole numbers.

\begin{tabular}{|c|c|c|c|}
\hline Property Name & Property Rule/Summary & Example & $\begin{array}{c}\text { Does it hold } \\
\text { for Subtraction? }\end{array}$ \\
\hline Commutative & $a+b=b+a$ & $\begin{array}{l}7-3=4 \\
3-7=-4\end{array}$ & \\
\hline Identity & $\begin{array}{l}a+0=a \\
0+a=a\end{array}$ & $\begin{array}{l}74,831-0=74,831 \\
0-74,831=-74,831\end{array}$ & $\begin{array}{l}\text { Not } \\
\text { Quite! }\end{array}$ \\
\hline Associative & $(a+b)+c=a+(b+c)$ & $\begin{array}{l}(12-5)-2=7-2=5 \\
12-(5-2)=12-3=9\end{array}$ & \\
\hline Closure & $\begin{array}{c}a+b=c \\
\text { is a whole \# }\end{array}$ & $\begin{array}{c}17-30=-13 \\
\text { Not a whole number }\end{array}$ & No! \\
\hline
\end{tabular}


Which Properties of Whole Number Addition Hold for Whole Number Multiplication?

Let $a, b$, and $c$ be whole numbers.

\begin{tabular}{|c|c|c|c|}
\hline Property Name & $\begin{array}{c}\text { Property } \\
\text { Rule/Summary }\end{array}$ & Example & $\begin{array}{c}\text { Does the Property Hold } \\
\text { for Multiplication? }\end{array}$ \\
\hline Commutative & & & \\
\hline Identity & & & \\
\hline Associative & & & \\
\hline Closure & & & \\
\hline
\end{tabular}

Are there any other properties that we have only for Multiplication?

\begin{tabular}{|c|c|c|c|}
\hline Property Name & Property Rule & Property Summarized & Example \\
\hline $\begin{array}{c}\text { Multiplication by } \\
\text { Zero Property }\end{array}$ & & & \\
\hline Distributive & & & \\
Property of & & & \\
Multiplication & & & \\
over Addition & & & \\
\hline
\end{tabular}

EXAMPLE OF APPROPRIATELY COMPLETED CHART (NEXT PAGE) 
Which Properties of Whole Number Addition Hold for Whole Number Multiplication? Let $a, b$, and $c$ be whole numbers.

\begin{tabular}{|c|c|c|c|}
\hline Property Name & $\begin{array}{c}\text { Property } \\
\text { Rule/Summary }\end{array}$ & Example & $\begin{array}{l}\text { Does the Property Hold } \\
\text { for Multiplication? }\end{array}$ \\
\hline Commutative & $\begin{array}{l}\text { For }+: \\
a+b=b+a\end{array}$ & $\begin{array}{l}3 \cdot 4=4+4+4=12 \\
4 \cdot 3=3+3+3+3=12\end{array}$ & $\begin{array}{l}\text { Yes! } \\
\qquad a \cdot b=b \cdot a\end{array}$ \\
\hline Identity & $\begin{array}{l}\text { For }+: \\
\qquad \begin{array}{l}a+0=a \\
0+a=a\end{array}\end{array}$ & $\begin{array}{ll}17 \cdot 0=0 & \text { No... } \\
17 \cdot 1=17 & \text { yes! } \\
1 * 17=17 & \text { yes! }\end{array}$ & $\begin{array}{l}\text { Yes, but for } 1 \\
1^{*} a=a \cdot 1=a \\
1 \text { is the "multiplicative identity" }\end{array}$ \\
\hline Associative & $\begin{array}{l}\text { For }+: \\
(a+b)+c=a+(b+c)\end{array}$ & $\begin{array}{l}(4 \cdot 3) \cdot 2=12 \cdot 2=24 \\
4 \cdot(3 \cdot 2)=4 \cdot 6=24\end{array}$ & $\begin{array}{c}\text { Yes! } \\
(a \cdot b) \cdot c=a \cdot(b \cdot c)\end{array}$ \\
\hline Closure & $\begin{array}{l}\text { For }+ \text { : } \\
\qquad \begin{array}{l}a+b=c \\
\tau\end{array} \\
\text { is a whole number }\end{array}$ & $\begin{array}{l}1758 \times 3718342 \\
\text { whatever it is, it's a } \\
\text { whole number! }\end{array}$ & $\begin{array}{l}\text { Yes! } \\
a \cdot b=c \\
\text { is a whole number }\end{array}$ \\
\hline
\end{tabular}

Are there any other properties that we have only for Multiplication?

\begin{tabular}{|c|c|l|c|}
\hline Property Name & Property Rule & Property Summarized & Example \\
\hline $\begin{array}{c}\text { Multiplication by } \\
\text { Zero Property }\end{array}$ & $a \cdot 0=0$ & $\begin{array}{l}\text { The product of any } \\
\text { whole number and zero } \\
\text { is zero. }\end{array}$ & $37,342,897 \cdot 0=0$ \\
\hline $\begin{array}{c}\text { Distributive } \\
\text { Property of } \\
\text { Multiplication } \\
\text { over Addition }\end{array}$ & $a(b+c)=a \cdot b+a \cdot c$ & $\begin{array}{l}\text { We can distribute } \\
\text { multiplication over } \\
\text { addition or subtraction }\end{array}$ & $3(4+7)=3(11)=33$ \\
\hline
\end{tabular}

Note: $a(b c) \neq a b \cdot a c \quad$ Multiplication does not distribute over multiplication.

$$
\text { Example: } \quad \begin{aligned}
& 5(3 \cdot 2)=5(6)=30 \\
& 5 \cdot 3+5 \cdot 2=15+10=25
\end{aligned}
$$


Which Properties of Whole Number Multiplication Hold for Whole Number Division?

Let $a, b$, and $c$ be whole numbers.

\begin{tabular}{|c|c|c|c|}
\hline Property Name & $\begin{array}{c}\text { Property Rule/ } \\
\text { Summary }\end{array}$ & Example & $\begin{array}{c}\text { Does the } \\
\text { Property Hold } \\
\text { for Division? }\end{array}$ \\
\hline Commutative & & & \\
\hline Identity & & & \\
\hline Associative & & & \\
\hline Closure & & & \\
\hline Zero Property & & & \\
\hline Distributive Property & & & \\
\hline
\end{tabular}

\section{EXAMPLE OF APPROPRIATELY COMPLETED CHART}

Which Properties of Whole Number Multiplication Hold for Whole Number Division? Let $a, b$, and $c$ be whole numbers.

\begin{tabular}{|c|c|c|c|}
\hline Property Name & $\begin{array}{l}\text { Property Rule/ } \\
\text { Summary }\end{array}$ & Example & $\begin{array}{l}\text { Does the Property } \\
\text { Hold for Division? }\end{array}$ \\
\hline Commutative & $\begin{array}{l}\text { For } \times \text { : } \\
a \times b=b \times a\end{array}$ & $\begin{array}{l}12 \div 4=3 \\
4 \div 12=1 / 3\end{array}$ & NO! \\
\hline Identity & $\begin{array}{l}\text { For } \times \text { : } \\
a \times 1=1 \times a=a\end{array}$ & $\begin{array}{ll}4 \div 1=4 & \checkmark \\
1 \div 4=1 / 4 & \times\end{array}$ & Not Quite! \\
\hline Associative & $\begin{array}{l}\text { For } \times \text { : } \\
a \times(b \times c)=(a \times b) \times c\end{array}$ & $\begin{array}{l}12 \div(6 \div 3)=6 \\
(12 \div 6) \div 3=2 / 3\end{array}$ & NO! \\
\hline Closure & $\begin{array}{l}\text { For } \times: \quad a, b, c \text { are } \\
\text { all whole } \\
a \times b=c \text { numbers }\end{array}$ & $\begin{array}{l}\text { Counterexample } \\
\qquad \begin{aligned} 4 \div 12 & =1 / 3 \\
1 / 3 & \text { is not a whole number }\end{aligned}\end{array}$ & No! \\
\hline Zero Property & $\begin{array}{l}\text { For } \times: \\
a \times 0=0 \times a=0\end{array}$ & $\begin{array}{l}12 \div 0 \text { is undefined } \times \\
0 \div 12=0\end{array}$ & Not Quite! \\
\hline \multirow{2}{*}{$\begin{array}{l}\text { Distributive } \\
\text { Property }\end{array}$} & $\begin{aligned} a & \times(b+c) \\
& =a \times b+a \times c\end{aligned}$ & $\begin{array}{l}12 \div(3+9)=1 \\
(12 \div 3)+(12 \div 9) \neq 1\end{array}$ & \multirow[t]{2}{*}{ Not Quite! } \\
\hline & $\begin{aligned} a \times b & +a \times c \\
& =a \times(b+c)\end{aligned}$ & $\begin{array}{l}(3+9) \div 12=1 \\
(3 \div 12)+(9 \div 12)=1\end{array}$ & \\
\hline
\end{tabular}




\section{APPENDIX E: MT1 COURSE SCHEDULE}

\begin{tabular}{|c|c|c|c|}
\hline Week & Session \# & Topics Covered & Assignments, Assessments, Activities \\
\hline \multirow{2}{*}{1} & 1 & Introduction & \\
\hline & 2 & Problem Solving & \\
\hline \multirow{2}{*}{2} & 3 & Problem Solving, & Homework 1 due \\
\hline & 4 & Ancient Numerals & \\
\hline \multirow{2}{*}{3} & 5 & Ancient Numerals & Homework 2 due \\
\hline & 6 & Non-decimal Number Systems & Quiz 1 \\
\hline \multirow{2}{*}{4} & 7 & \multicolumn{2}{|c|}{ Pre-Teaching Assessment for Dissertation Study was issued } \\
\hline & 8 & Set Theory, Venn Diagrams & Homework 3 due \\
\hline \multirow{2}{*}{5} & 9 & Catch Up/ Review for Exam 1 & \\
\hline & 10 & \multicolumn{2}{|c|}{ Exam 1: Problem Solving through Venn Diagrams } \\
\hline \multirow{2}{*}{6} & 11 & Addition of Whole Numbers & Addition Properties \\
\hline & 12 & Subtraction of Whole Numbers & \\
\hline \multirow[t]{2}{*}{7} & 13 & Multiplication of Whole Numbers & $\begin{array}{l}\text { Multiplication Properties, } \\
\text { Distributive Property }\end{array}$ \\
\hline & 14 & Division of Whole Numbers & \\
\hline \multirow{2}{*}{8} & 15 & Divisibility and Factorization & Homework 4 due \\
\hline & 16 & GCF and LCM & Quiz 4 \\
\hline \multirow{2}{*}{9} & 17 & Catch Up/ Review for Exam 2 & \\
\hline & 18 & \multicolumn{2}{|c|}{ Exam 2: Whole Number Arithmetic through GCF and LCM } \\
\hline \multirow{2}{*}{10} & 19 & Integer Models and Arithmetic & \\
\hline & 20 & Integer Models and Arithmetic & The Role of Properties, Homework 5 due \\
\hline \multirow{2}{*}{11} & 21 & Fraction Models \& Arithmetic & \\
\hline & 22 & Fraction Addition/Subtraction & The Role of Properties, Homework 6 due \\
\hline \multirow{2}{*}{12} & 23 & Fraction Multiplication/Division & \\
\hline & 24 & Decimal Numbers \& Arithmetic & The Role of Properties, Homework 7 due \\
\hline \multirow{2}{*}{13} & 25 & Decimal Arithmetic & Quiz 5, Exam 3 Review issued \\
\hline & 26 & \multicolumn{2}{|c|}{ Post-Teaching Assessment for Dissertation Study was issued } \\
\hline \multirow{2}{*}{14} & 27 & \multicolumn{2}{|c|}{ Exam 3: Integers, Fractions, and Decimals } \\
\hline & 28 & \multicolumn{2}{|c|}{ Thanksgiving Day } \\
\hline \multirow[t]{2}{*}{15} & 29 & Catch Up/ Review for Final Exam & \\
\hline & 30 & Catch Up/ Review for Final Exam & \\
\hline $\begin{array}{l}\text { Finals } \\
\text { Week }\end{array}$ & \multicolumn{3}{|c|}{ FINAL EXAM } \\
\hline
\end{tabular}




\section{APPENDIX F: LESSON HANDOUTS FOR MT1 PETS}

\section{Addition of Whole Numbers}

\section{Ways of Modeling Addition}

Set Model of Addition - Let $a$ and $b$ be any two whole numbers. If $A$ and $B$ are any two disjoint sets for which $a=n(A)$ and $b=n(B)$, then the sum of $a$ and $b$, written $a+b$ is given by $a+$ $b=n(A \cup B)$.

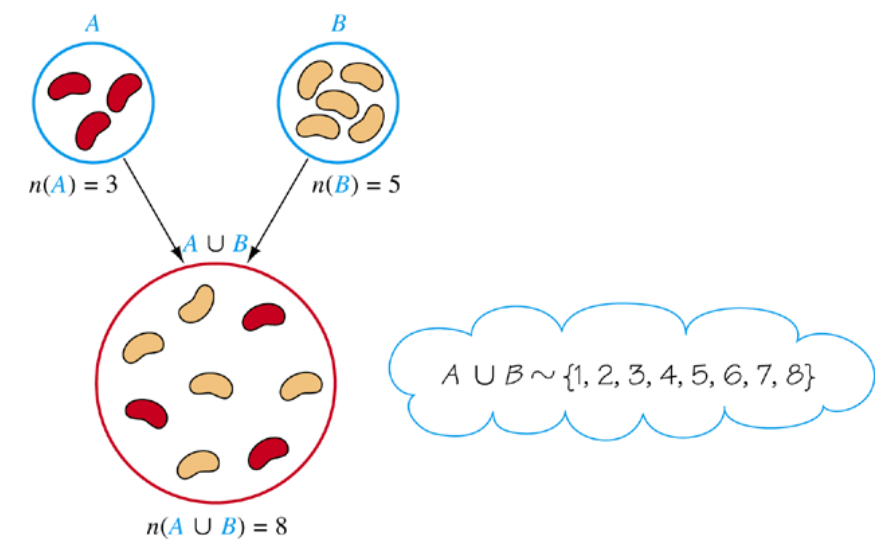

Number-Line (Measurement) Model of Addition

- On a Number Line, whole numbers are geometrically interpreted as distances.

- Addition can be visualized as combining two distances to get a total distance.

- Individual distances do not overlap to combine to the total distance.

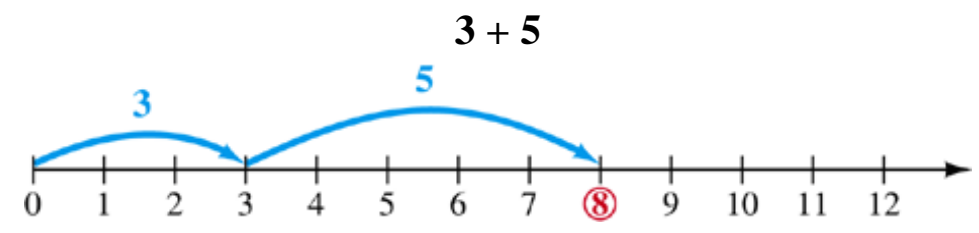

\section{Strategies/Algorithms for Whole Number Addition}

- Using the standard algorithm

- Using Base 10 blocks

Compute the following sum using the standard algorithm. Then model each step of the algorithm with Base 10 Blocks. Illustrate the blocks on your paper.

$$
139+273
$$


- Other Addition algorithms

- Lattice method for addition

○ Cross-Out/Scratch-Up Algorithm

The standard algorithm for addition may be familiar to you, but the others may be unfamiliar. Children in elementary classrooms may learn several strategies for addition. Students are also capable of inventing their own algorithms for addition. It is important for you as future teachers to learn about the different methods and to compare and contrast these methods when completing the same addition problem.

As we explore different strategies for adding whole numbers, let's evaluate each strategy based on the following: accuracy, generality, efficiency, ease of accurate use, and transparency. You will be tasked with explaining and justifying why the strategies work. Strategies can be justified by identifying the role of place value and/or ascertaining the number property applied.

\section{Classroom Tasks (Whole Number Addition)}

DIRECTIONS: Get into groups of three or four and work on the following tasks. Discuss the questions, work out what is asked, determine the meaning of each, and answer appropriately.

1. Use a number line to find the sum of $5+3$ and $3+5$. What do you find? What can you conjecture about the addition of whole numbers?

Let $a$ and $b$ be two unique whole numbers. Use a number line to find the sum of $a+b$ and $b+a$ ? What do you find? Repeat this previous step a few more times with different numbers for $a$ and $b$. What can you conclude about the addition of whole numbers?

2. What would be a quick way of determining the value of $99+234+1$ without using a calculator or writing anything down? What did you do and is it related to what you concluded about the addition of whole numbers from above?

3. If $a=12, b=6$ and $c=2$, what are the values of each of the following?

$(a+b)+c$

$a+(\mathrm{b}+c)$

Repeat this, only this time let $a=51, b=26$, and $c=12$. What do you find? What can you conjecture about the addition of whole numbers?

Repeat again, only this time let a, b, and $\mathrm{c}$ be any whole numbers that your group chooses. What do you find? What can you conclude about the addition of whole numbers?

4. Do you think $a+b$ will always equal $b+\mathrm{a}$, no matter which numerical values you allow $a$ and $b$ to be? Do you think $a+(b+c)$ will always equal $(a+b)+c$, no matter which 
numerical values you allow $a, b$ and $c$ to be?

5. In adding 957 and 384, will the strategy of adding 1200, 130, and 11 give the right answer? If so, why? Discuss the role of place value and whether any addition property is used.

6. You may have heard in your prior mathematics education that "when you add number and zero, the result is the number itself." Have you heard this or some similar statement? What does it mean to you? Is it true always? How can we state this concisely?

7. Is the sum of two whole numbers also a whole number? Is this always true for any two whole numbers? Is this always true when adding three or more whole numbers?

\section{Summary of Properties of Whole Number Addition}

Properties of Whole Number Addition - Let $a, b$, and $c$ be whole numbers.

\begin{tabular}{|l|l|l|l|}
\hline Property & Property Rule & Property Summarized & Example \\
\hline Commutative & & & \\
\hline Identity & & & \\
\hline Associative & & & \\
\hline Closure & & & \\
\hline
\end{tabular}

\section{Questions on the Homework pertaining Addition Properties}

1. Which property allows you to quickly state the value of $m$ in the following equation: $37+44=44+m ?$

2. How can you quickly determine the value of the following numerical expressions? Which property (or properties) would you be using?

a. $102+798$

b. $89+101+10$ 
c. $990+(10+435)$

3. Add $462+835$ using three different numerical strategies. Explain why each of your strategies is valid by making connections to place value and whether number properties are applied.

\section{Subtraction of Whole Numbers}

\section{Understanding Subtraction}

Subtraction of Whole Numbers - Let $a$ and $b$ be two whole numbers. The difference of $a$ and $b$, written $a-b$, is the unique whole number $c$ such that $a=b+c$. That is, $a-b=c$ if and only if there is a whole number $c$ such that $a=b+c$.

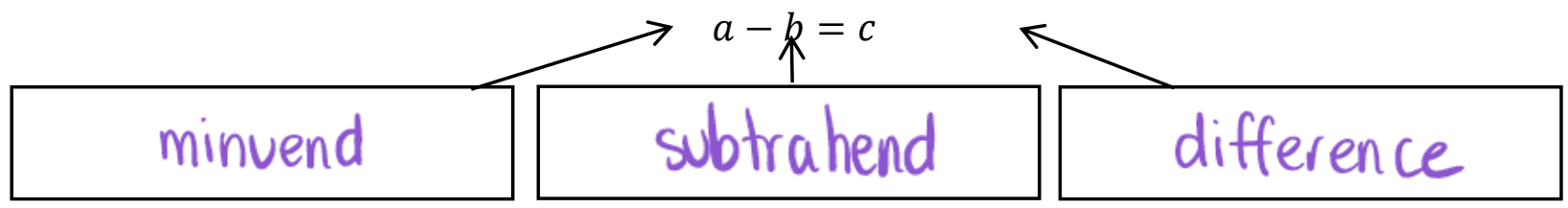

Relationships between Addition and Subtraction:

- Knowing facts about addition produces subtraction facts: $5+3=8 \longrightarrow 8-5=3$
These are called "fact families".

\section{Ways of Modeling Subtraction}

Subtraction can be illustrated using many different models. In order to use subtraction fluently in mathematical problem solving, students must become familiar with $\boldsymbol{A} \boldsymbol{L} \boldsymbol{L}$ of these models.

For each of the following models, we will illustrate the subtraction problem: $\mathbf{8}-\mathbf{5}$.

1) Take Away Model

- Start with 8 objects.

- Take away 5 objects.

- How many objects are left?

2) Missing-Addend (Adding Up) Model

- Start with 5 objects.

- How many more objects are needed to give a total of 8 objects?

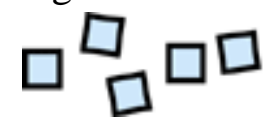

3) Comparison Model

- Start with two collections, with 8 objects in one collection and 5 in the other. 
- How many more objects are in the larger collection?

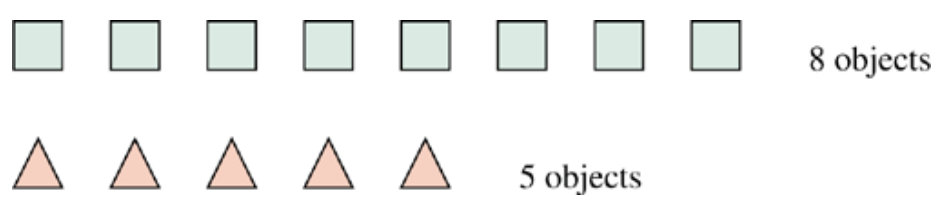

4) Number-Line (Measurement) Model

- On a Number Line, whole numbers are geometrically interpreted as distances.

- Subtraction can be visualized as the distance between two values, similar to the MissingAddend Model.

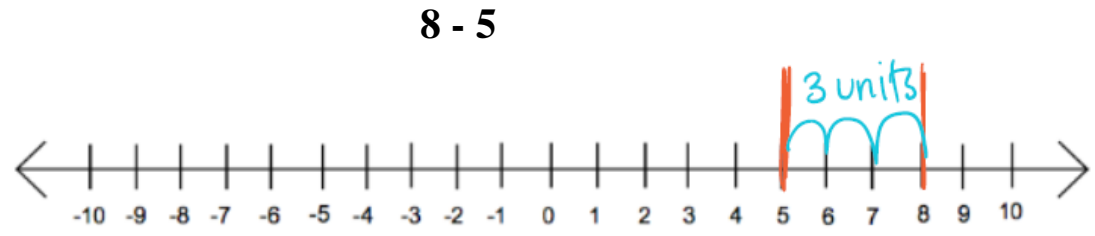

- In more physical terms, on a number line, subtraction can be viewed as "jumping" between values, similar to the Take-Away Model.

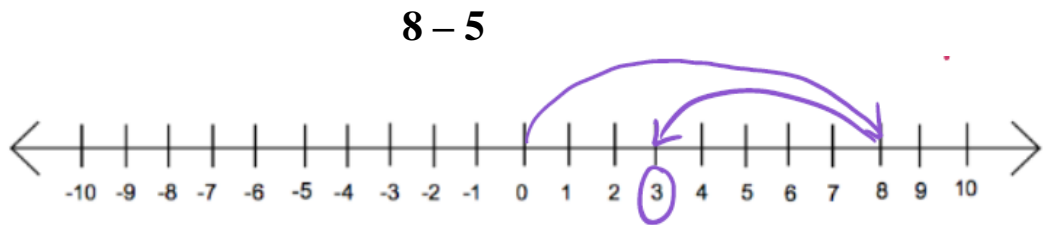

\section{Strategies/Algorithms for Whole Number Subtraction}

- Using the standard algorithm

- Using Base 10 blocks

Compute the following difference using the standard algorithm. Then model each step of the algorithm with Base 10 Blocks. Illustrate the blocks on your paper.

$$
300-148
$$

- Other Subtraction algorithms
- Adding/Counting Up Algorithm
○ Indian/Cross-Out Algorithm
○ European Algorithm

The standard algorithm for subtraction may be familiar to you, but the others may be unfamiliar. Children in elementary classrooms may learn several strategies for subtraction. Students are also 
capable of inventing their own algorithms for subtraction. It is important for you as future teachers to learn about the different methods and to compare and contrast these methods when completing the same subtraction problem.

As we explore different strategies for subtracting whole numbers, let's evaluate each strategy based on the following: accuracy, generality, efficiency, ease of accurate use, and transparency. You will be tasked with explaining and justifying why the strategies work. Strategies can be justified by making connections to place value and by ascertaining whether a number property is applied.

\section{Classroom Tasks (Whole Number Subtraction)}

DIRECTIONS: Get into groups of three or four and work on the following tasks. Discuss the questions, work out what is asked, determine the meaning of each, and answer appropriately.

1. Let $a$ and $b$ be two unique whole numbers. Use a number line to find the value of $a-b$ and

$b-a$ ? What do you find? Repeat this with different numbers for $a$ and $b$. What can you say about the subtraction of whole numbers? Contrast this with what you found regarding addition of whole numbers?

2. If $a=12, b=6$ and $c=2$, what are the values of each of the following?

$(a-b)-c$

$a-(\mathrm{b}-c)$

Repeat this, only this time let $a=51, b=26$, and $c=12$. What do you find? What can you conjecture about the addition of whole numbers?

Repeat again, only this time let a, b, and c be any whole numbers that your group chooses. What do you find? What can you say about the subtraction of whole numbers? Contrast this with what you found regarding addition of whole numbers?

3. In subtracting 214 and 148, will the strategy of adding 100, -30 , and -4 give the right answer? If so, why?

4. Explain why subtracting 2462 from 4835 is the same as

a. Subtracting 2432 from 4835 , and then subtracting 30 more.

b. Subtracting 2462 from 4862, and then subtracting 27 more.

c. Subtracting 2400 from 4800 and then subtracting 27 more.

5. Previously, we saw that $a+0=0+a=a$ for any $a$ that is a whole number. Now, let $a$ be any whole number that your group chooses? What is the value of $a-0$ ? What is the value of $0-a$ ? What can you say about subtraction and contrast it to what you concluded about addition? 
6. Let $a$ and $b$ be two unique whole numbers. Is the value of $a-b$ always a whole number? Explain. Contrast it what you found regarding addition.

Which Properties of Whole Number Addition Hold for Whole Number Subtraction? Let $a, b$, and $c$ be whole numbers.

\begin{tabular}{|l|l|l|l|}
\hline Property Name & Property Rule/Summary & Example & $\begin{array}{c}\text { Does it hold for } \\
\text { Subtraction? }\end{array}$ \\
\hline Commutative & & & \\
\hline Identity & & & \\
\hline Associative & & & \\
\hline Closure & & & \\
\hline
\end{tabular}

\section{Multiplication of Whole Numbers}

Multiplication of Whole Numbers as Repeated Addition - Let $a$ and $b$ be any two whole numbers. Then the product of $a$ and $b$, written as $a \cdot b$, is defined by

$$
\begin{gathered}
a \cdot b=b+b+b+b+\cdots+b \text { when } a \neq 0 \\
\text { and by } 0 \cdot b=0
\end{gathered}
$$

\section{Note about order:}

Read $a \cdot b$ aloud as " $a$ copies of $b$ ", i.e. $\underbrace{b+b+b+\ldots+b}_{a \text { times }}$.

We know this is the same value as $b \cdot a=\underbrace{a+a+a+\ldots+a}_{b \text { times }}$, but we want to have different models for e.g. $2 \cdot 3=3+3$ versus $3 \cdot 2=2+2+2$.

Multiplication can be illustrated using many different models.

\section{1) Array Model}

Lisa had to plant 5 rows of bean seeds with each row containing 8 seeds.

How many seeds did she plant?

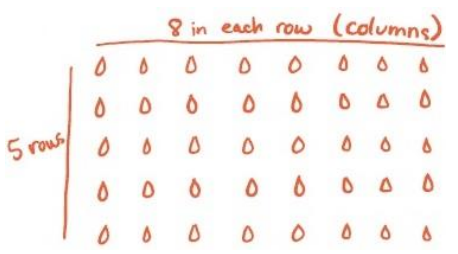


2) Skip-Count Model

Example: $5 \cdot 3$

$5 \cdot 3$

- Start at 0

- Skip to 3

- $\quad$ Skip to 6

- $\quad$ Skip 3 spaces, 5 times

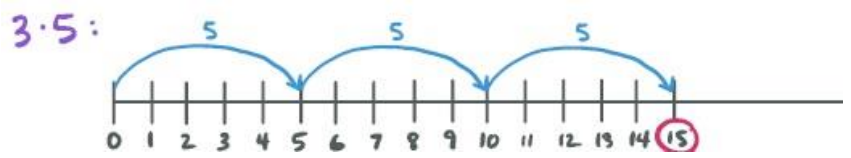

3) Multiplication Tree Model

Melissa has a box of 4 flags, colored red, yellow, green, and blue. How many ways can she display 2 of the flags on a flagpole?

4 first choices $\times 3$ second choices $=$

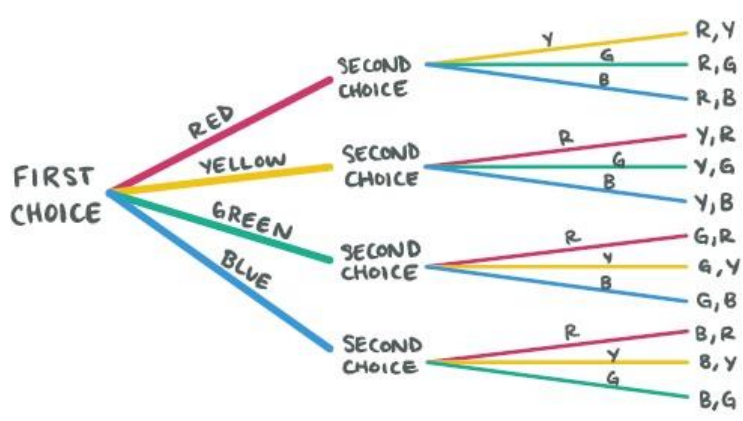
12 ways

4) Rectangular Area Model

Janet wants to order square ceramic tiles to cover the floor of her $4 \mathrm{ft}-b y-3 \mathrm{ft}$ hall space. If the tiles are each 1 square foot, how many will see need to order?

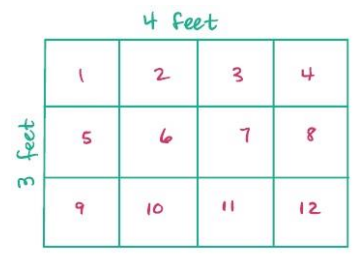

Which Properties of Whole Number Addition Hold for Whole Number Multiplication?

Let $a, b$, and $c$ be whole numbers.

\begin{tabular}{|c|c|c|c|}
\hline Property Name & $\begin{array}{c}\text { Property } \\
\text { Rule/Summary }\end{array}$ & Example & $\begin{array}{c}\text { Does the Property Hold } \\
\text { for Multiplication? }\end{array}$ \\
\hline Commutative & & & \\
\hline Identity & & & \\
\hline Associative & & & \\
\hline Closure & & & \\
\hline
\end{tabular}


Are there any other properties that we have only for Multiplication?

\begin{tabular}{|c|c|c|c|}
\hline Property Name & Property Rule & Property Summarized & Example \\
\hline $\begin{array}{c}\text { Multiplication by } \\
\text { Zero Property }\end{array}$ & & & \\
\hline $\begin{array}{c}\text { Distributive } \\
\text { Property of }\end{array}$ & & & \\
Multiplication \\
over Addition
\end{tabular}

Students are capable of inventing their own algorithms for multiplication. However, sometimes their limited understanding of the meaning of the Base Ten system causes them to invent algorithms that are not mathematically valid. Most common algorithms and strategies for multiplication rely on the use of the distributive property.

\section{Strategies/Algorithms for Whole Number Multiplication}

- Using the standard algorithm for multiplication

Find the value of $90 \times 34$ using the standard multiplication algorithm. Why does this algorithm work? Does the algorithm rely on any number property?

- Partial Products/Area Model Algorithm

Find the value of $90 \times 34$ using the Partial Products/Area Model algorithm. Does the algorithm rely on any number property? Compare and contrast this algorithm with the standard algorithm for multiplication.

- Other Multiplication Algorithms

○ Lattice Algorithm for Multiplication

○ Russian-Peasant/Double-Half Algorithm

\section{Classroom Tasks (Whole Number Multiplication \& Properties)}

DIRECTIONS: Get into groups of three or four and work on the following tasks. Discuss the questions, work out what is asked, determine the meaning of each, and answer appropriately.

11. Without using a calculator or relying on any algorithm, compute each of the following products in at least three different ways. State any properties that you use.

a. $24 \times 25$

b. $120 \times 325$ 
12. How could you use the stated property to evaluate $12 \times 250$ ?

a. How could you use an associative property to find the value of $12 \times 250$ ?

b. How could you use the distributive property to find the value of $12 \times 250$ ?

13. How could you use the distributive property to find the value of $17 \times 97+17 \times 3$ ?

14. Which property would you use to find the value of $p$ ? $16 \times 28=11 \times 28+p \times 28$.

15. How would you quickly determine the value of $25 \times 37 \times 4$ ? Which property (or properties) would you be applying?

\section{Questions on the Homework pertaining Multiplication Properties}

1. Without using a calculator or relying on any algorithm, compute each of the following products in at least three different ways. State any properties that you use.
a. $16 \times 25$
b. $16 \times 35$

2. Solve for $m$ only by applying a number property (or properties)? DO NOT use algebraic manipulation.
a. $15 \times 13=9 \times 13+m \times 13$
b. $16 m=8 m+32$

\section{Division of Whole Numbers}

\section{Ways of Modeling Division}

1) Repeated Subtraction Model

The repeated subtraction model can be realized easily with physical objects; the process is called division bu aroupina.

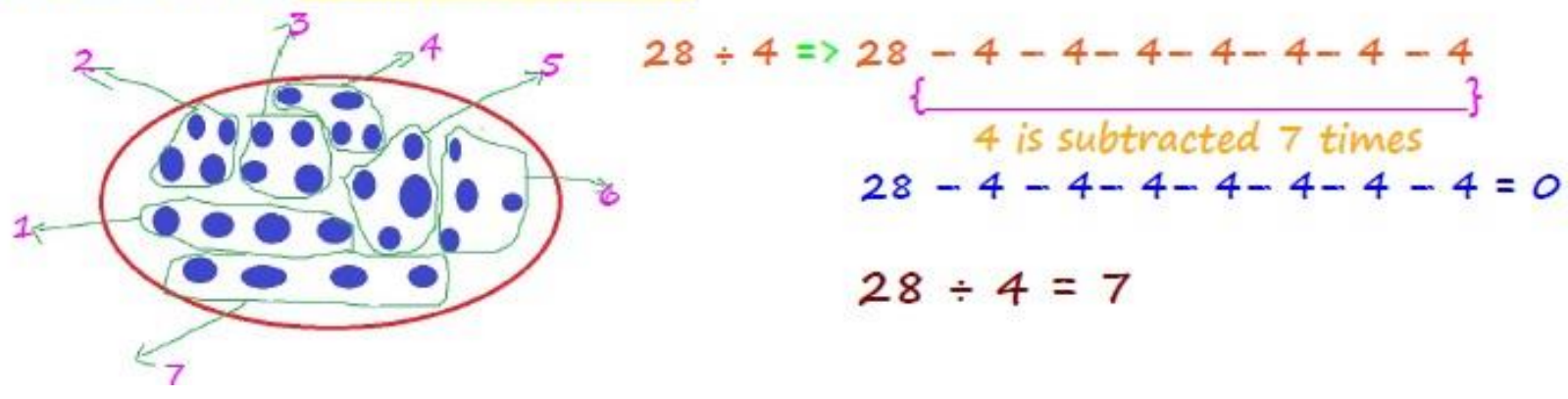


2) Partition Model

The partition model is also realized easily with physical objects, in which case the process is called division by equal sharing or partitive division model.

\begin{tabular}{|c|}
\hline$x \times \times \times \times \times x$ \\
\hline$x \times \times \times \times \times x$ \\
\hline$\times \times \times \times \times \times x$ \\
\hline$x \times \times \times \times \times x$ \\
\hline
\end{tabular}

Division as a partition: $28 \div 4=7$ because when 28 objects are partitioned into 4 equal-sized sections, there are 7 objects in each partition.

3) Missing Factor Model

In the repeated-subtraction model, $28 \div 4=7$ because seven groups are formed when $4 s$ are subtracted repeatedly. That is,

$$
28=4+4+4+4+4+4+4=7 \times 4 \text {. }
$$

However, in the partition model, $28 \div 4=7$ because

$$
28=7+7+7+7=4 \times 7 \text {. }
$$

In both cases, the division $28 \div 4$ can be viewed as finding the factor $c$ for which

$$
28=4 \times c \text { or } 28=c \times 7 \text {. }
$$

The missing-factor model is usually the concept adopted to define division formally.

\section{Defining Division}

Division of Whole Numbers - Let $a$ and $b$ be Whole Numbers with $b \neq 0$, then $a \div b=c$ if and only if $a=b \cdot c$ for a unique Whole Number $c$.

- This is based off of the Missing Factor Model

- This illustrates the relationship back to multiplication which in turn shows the relationship to addition.

Division Vocabulary:

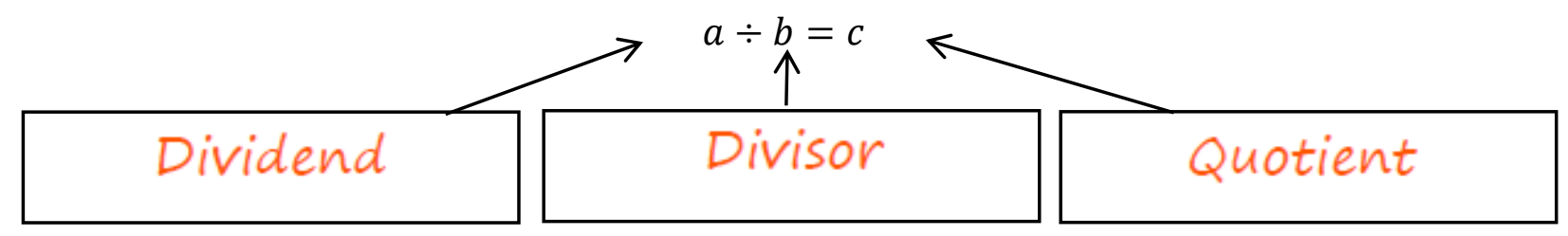


What if $a \div b$ is not a whole number? In this case, we will have a remainder.

Notation for remainders: Consider $11 \div 4$.

$$
11 \div 4=2 r 3
$$

$11=2 \times 4+3$

It is common to write $a \div b=q R r$

if $a=q \times b+r, O \leq r<b$

Which Properties of Whole Number Multiplication Hold for Whole Number Division?

Let $a, b$, and $c$ be whole numbers.

\begin{tabular}{|c|c|c|c|}
\hline Property Name & $\begin{array}{c}\text { Property Rule/ } \\
\text { Summary }\end{array}$ & Example & $\begin{array}{c}\text { Does the Property } \\
\text { Hold for Division? }\end{array}$ \\
\hline Commutative & & & \\
\hline Identity & & & \\
\hline Associative & & & \\
\hline Closure & & & \\
\hline $\begin{array}{c}\text { Zero Property } \\
\text { Distributive } \\
\text { Property }\end{array}$ & & & \\
\hline
\end{tabular}

\section{Strategies/Algorithms for Whole Number Division}

- Using the standard algorithm for Division

- Dividing by Subtracting Powers of Ten

- Scaffolding Algorithm

Compute the following by (a) using the standard algorithm for division, (b) subtracting powers of ten, and (c) using the scaffolding algorithm.

$$
462 \div 3
$$

\section{Classroom Tasks (Whole Number Division)}

DIRECTIONS: Get into groups of three or four and work on the following tasks. Discuss the questions, work out what is asked, determine the meaning of each, and answer appropriately. 
Question 1: Applewood Elementary School has just bought 24 computers for its 4 fifth-grade classes. How many computers will each classroom get? Use a diagram or a picture to illustrate your answer. Describe in words how you arrived at your answer.

Question 2: Carlos has 24 apples with which to make apple pies. If it takes 4 apples per pie, how many pies can he bake? Use a diagram or a picture to illustrate your answer. How might your reasoning for this problem differ from Question 1? Explain your method. 


\section{Integer Operations}

Integers: The integers, $Z=\{\ldots-3,-2,-1,0,1,2,3, \ldots\}$ consists of:

- The Natural Numbers $N=\{1,2,3,4, \ldots$.$\} now called the positive integers$

- The negative numbers $\{\ldots,-3,-2,-1\}$ and

- The number zero which is neither positive nor negative

Absolute Value: If $n$ is an integer, then its absolute value is the nonnegative integer $|n|$ given by:

$$
|n|=\left\{\begin{array}{l}
n \text { if } \mathrm{n} \text { is positive or } 0 \\
-n \text { if } \mathrm{n} \text { is negative }
\end{array}\right.
$$

Opposite/Negative of a Number - Pairs of numbers whose absolute values are equal.

Additive Inverse - The opposite of any integer $n$ such that $n+(-n)=0=(-n)+n$.

This is the inverse property of addition: the sum of a number and its opposite equals 0 .

\section{Representing Integers - Number Line Model}

We can continue the number line in both directions to represent positive and negative numbers.

Example: Plot $2,-3,0$, and -5 on one number line below.

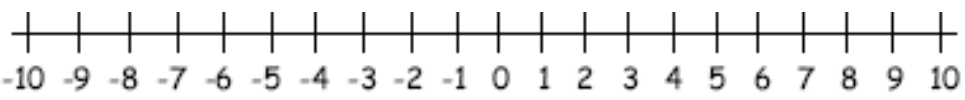

\section{Integer Addition}

We will use the number line model to illustrate addition of integers. This model can be used to develop rules for addition of integers.

In an elementary classroom, models are often physical in nature and are not typically drawn out in as much detail as we do here. However, to write complete lesson plans you'll need pictures of all of the stages of the model and verbal descriptions to tell you what to do.

\section{Number Line}

We often discuss number line models in terms of "walking along the number line." Don't forget-we ALWAYS start at zero!

1. View the following number line to see how to illustrate $5+3$ on the number line.

Describe the model in words. What is the solution? 


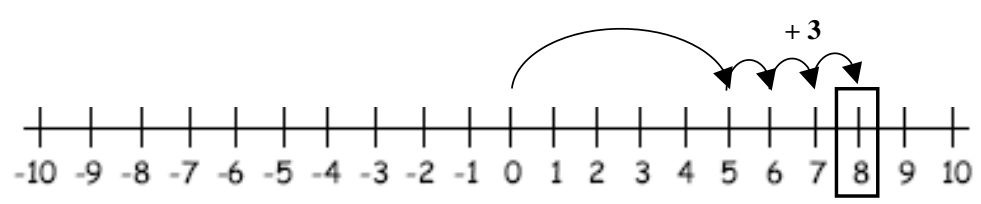

2. Use the number line to illustrate $-5+3$, and describe the model in words. What is the solution?

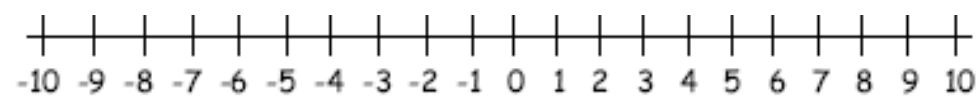

3. Use the number line to illustrate $5+(-3)$, and describe the model in words.

Remember, if you are adding a negative, you move backwards (left). What is the solution?

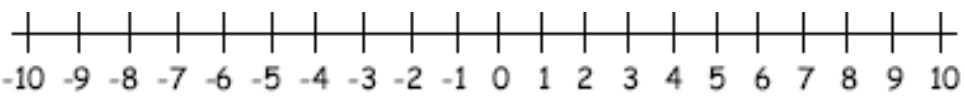

4. Use the number line to illustrate $(-5)+(-3)$, and describe the model in words. What is the solution?

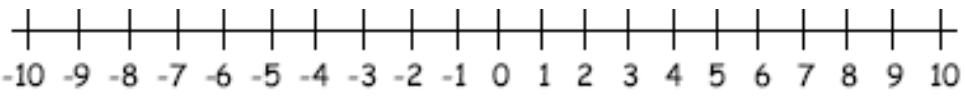

\section{Rules for Addition of Integers}

Fill in the table of your own explanation of how to add the two types of numbers stated. Use the models as a basis to develop a rule that can be used for all numbers (large and small).

\begin{tabular}{|c|c|c|c|}
\hline Number & Number & Example & Explanation of Rule \\
\hline+ & + & $4+3$ & \\
\hline+ & - & $4+(-3)$ or $5+(-7)$ & \\
\hline- & + & $(-4)+3$ or $(-5)+7$ & \\
\hline- & - & $(-4)+(-3)$ & \\
\hline
\end{tabular}




\section{Integer Subtraction}

We will use the number line model to illustrate subtraction of integers. This model can be used to develop rules for subtraction of integers.

\section{Number Line}

We often describe out number line models physically, using words like "facing to the right", "facing to the left", "walking forwards" or "walking backwards." There are a few ways to put these words together to come up with a coherent picture of integer addition/subtraction, but let's use the following model:

$>$ For addition, face to the right (towards infinity). For subtraction, face to the left.

$>$ If the number being added/subtracted is positive, walk forwards. If negative, walk backwards.

1. View the following number to line to see how to illustrate $7-3$ on the number line. Describe the model in words. What is the solution to $7-3$ ?

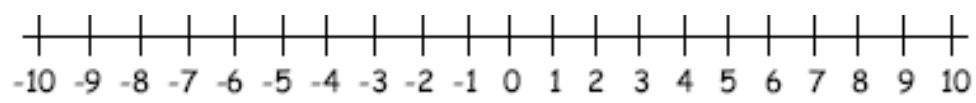

2. Use the number line to illustrate $-7-3$, and describe the model in words. What is the solution?

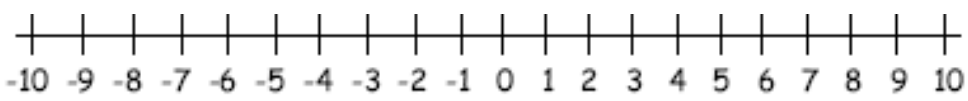

When faced with subtraction of a negative number, such as $7-(-3)$, you may have heard the phrase "keep change change." While this phrase produces the correct procedural results in this one specific type of problem, it does not help a student to understand why the subtraction above is equivalent to $7+3$. In order to build good number sense in our students, it's important to work with a variety of physical, visual, and verbal models for addition and subtraction of integers.

3. Use the number line to illustrate $7-(-3)$, and describe the model in words. What is the solution?

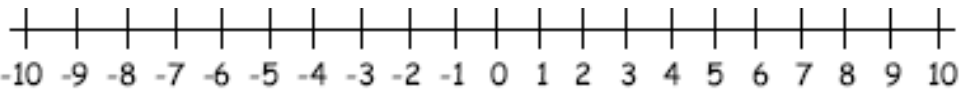

4. Use the number line to illustrate $(-7)-(-3)$. What is the solution?

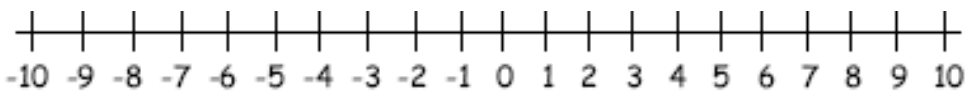




\section{Rules for Subtraction of Integers}

1. Using your results from the above models, rewrite the following subtraction problems as equivalent addition problems to yield the appropriate solution.
a) $3-4=-1$
b) $\quad-2-5=-7$
c) $\quad-7-(-3)=-4$
d) $6-4=2$

2. Hopefully you were successfully able to rewrite each of these subtraction problems as equivalent addition problems. All subtraction problems can be rewritten as such. Use this idea to help develop a general rule for subtraction of integers.

\section{Integer Multiplication}

We will use the number line to model multiplication of integers. This model can be used to explain rules for multiplication of integers.

\section{Number Line}

1. View the following to see how to illustrate $2 \cdot 4$ on the number line. Use the idea of repeated addition to illustrate the movement on the number line and the solution. $2 \cdot 4$ means the same thing as $4+4$ (or $2+2+2+2$ using the commutative property). Start at 0 .

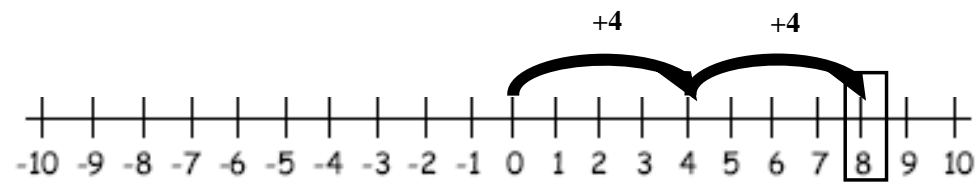

2. Use the number line to illustrate $2 \cdot(-4)$. What is the solution?

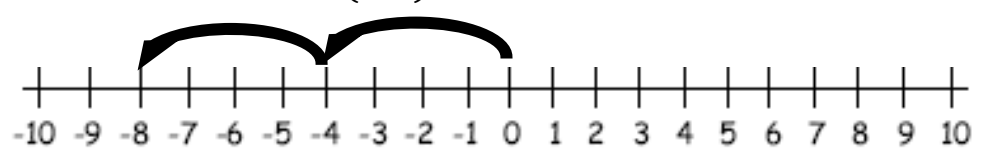


3. Use the number line to illustrate $(-2) \cdot 4$. What is the solution? Can you represent the solution on the number line?

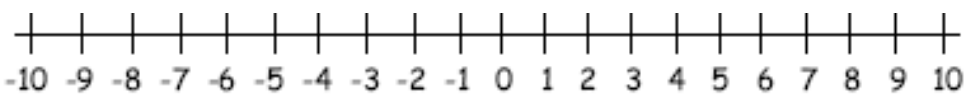

4. Use the number line to illustrate $(-2) \cdot(-4)$. What is the solution? Can you represent the solution on the number line?

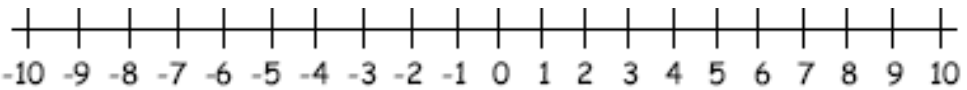

\section{Rules for Multiplication of Integers}

Fill in the table of your own explanation of how to multiply the two types of numbers stated. Use your results from the models above to help develop your rule. Use the models as a basis to develop a rule that can be used for all numbers (large and small).

\begin{tabular}{|c|c|c|c|}
\hline Number & Number & Example & Explanation of Rule \\
\hline+ & + & $4 \cdot 5=$ & \\
\hline- & - & $(-4) \cdot(-5)=$ & \\
\hline- & + & $(-4) \cdot 5=$ & \\
\hline+ & - & $4 \cdot(-5)=$ & \\
\hline
\end{tabular}

\section{Integer Division}

We will use the connection between multiplication and division to develop the rule for division of integers as the rules are similar. 


\section{$\underline{\text { Rules for Division of Integers }}$}

Fill in the table of your own explanation of how to divide the two types of numbers stated. Use your results from the table for Rules for Multiplication of Integers to help develop your rule.

\begin{tabular}{|c|c|c|c|}
\hline Number & Number & Example & Explanation of Rule \\
\hline+ & + & $8 \div 2=$ & \\
\hline- & - & $(-8) \div(-2)=$ & \\
\hline- & + & $(-8) \div 2=$ & \\
\hline+ & - & $8 \div(-2)=$ & \\
\hline
\end{tabular}

Working with negative integers can be quite a challenge for young students; your skill at facilitating their learning activities can be a major factor in determining whether something like "signed number" arithmetic is a struggle or something that becomes easy because the "rules" make sense. Memorizing rules is necessary for their efficient use, but understanding "why" they work makes using them much easier.

\section{Properties of Operations}

In the previous chapters we worked with the set of whole numbers, operating on whole numbers, and the properties of whole number operations. Now, we expand our number system to include "negative numbers" and form the set of integers. The set of integers is $Z=\{\ldots-3,-2,-1$, $0,1,2,3 \ldots\}$.

Can the properties of whole number operations be extended to the set of integers? 
Properties of Addition - Let $a, b$, and $c$ be integers.

\begin{tabular}{|l|l|l|l|}
\hline Property & Property Rule & Property Summarized & Example \\
\hline Commutative & & & \\
\hline Identity & & & \\
\hline Associative & & & \\
\hline Closure & \multicolumn{3}{|l|}{} \\
\hline \multicolumn{2}{|c|}{ We introduce another Property } & \\
\hline Inverse & \multicolumn{2}{|l}{} \\
\hline
\end{tabular}

Properties of Multiplication - Let $a, b$, and $c$ be integers.

\begin{tabular}{|c|l|l|l|}
\hline Property Name & Property Rule & Property Summarized & Example \\
\hline Commutative & & & \\
\hline Identity & & & \\
\hline Associative & & & \\
\hline Closure & & & \\
\hline $\begin{array}{c}\text { Multiplication } \\
\text { by Zero } \\
\text { Property }\end{array}$ & & & \\
\hline $\begin{array}{c}\text { Distributive } \\
\text { Property }\end{array}$ & & & \\
\hline
\end{tabular}




\section{Fractions}

\section{Basic Concepts of Fractions and Rational Numbers}

$\underline{\text { Fraction }}$ - A number whose value can be expressed as the quotient or ratio of any two numbers

$a$ and $b$ represented as $\frac{a}{b}$ where $b \neq 0$. The number $a$ is called the numerator. The number $b$ is called the denominator.

$$
\text { Examples of fractions: } \frac{-2}{3}, \frac{15}{65}, \frac{3.2}{6.4}, \frac{\pi}{4}
$$

$\underline{\text { Rational Number }}$ - A number whose value can be expressed as the quotient or ratio of two $\underline{\text { integers }} a$ and $b$, represented as $\frac{a}{b}$ where $b \neq 0$. The integer $a$ is called the numerator. The integer $b$ is called the denominator.

\section{Note:}

- In the list of fractions above, the first two, $\frac{-2}{3}$ and $\frac{15}{65}$, are rational numbers expressed as the quotient of two integers.

- The fraction $\frac{3.2}{6.4}$ is not expressed as the quotient of two integers, but it can be. How?

- The fourth fraction, $\frac{\pi}{4}$, is not a rational number. There is no way to express this fraction as a quotient of two integers.

\section{$\underline{\text { Representation of Fractions }}$}

A Fraction must clearly answer the following questions:

1. What is the unit? (What is the "whole"?)

2. Into how many equal parts (the denominator) has the unit been subdivided?

3. How many of these parts (the numerator) are under consideration?

Unit Fraction - A Rational Number written as a fraction where the numerator is one and the denominator is a positive integer.

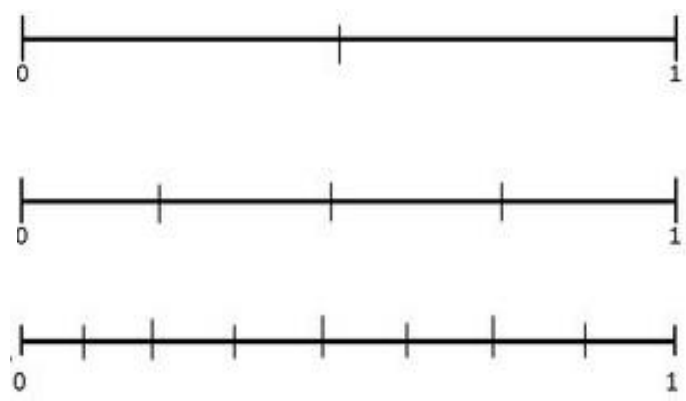




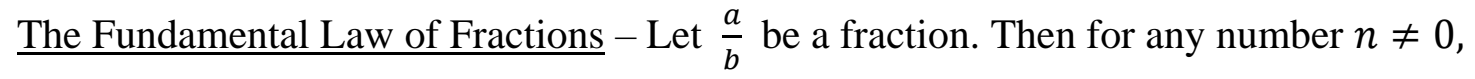

Note: This gives us a way to rewrite our example, $\frac{3.2}{6.4}=$

Cross Product Property of Equivalent Fractions -

NOTE:

A fraction $\frac{a}{b}$ is said to be in simplest form if $a$ and $b$ share no common factors other than 1 .

Models for Illustrating the Concept of Fraction

- Set Model

- Fraction Strips Model

- Fraction Circles Model

- Number Line Model

\section{Using Diagrams to Show the Equivalence of Fractions}

We are familiar with the idea that the two fractions $3 / 4$ and $6 / 8$ represent the same value. They are referred to as equivalent fractions. We will use diagrams, fraction circles, and Base 10 blocks to discuss the equivalence of fractions and then discuss how to write a fraction in its simplest form. If, over the years, you have had trouble understanding what is meant by equivalent fractions, you may find that working with the diagrams will alleviate some of your confusion.

\section{Methods for Expressing a Fraction in Simplest Form (Reducing a Fraction)}

Method \#1: Divide successively by common factors

Method \#2: Divide the numerator, $a$, and denominator, $b$, by $G C D(a, b)$. 
Method \#3: "Cancel out" or divide by common factors in the prime factorizations of $a, b$

\section{Types of Fraction Numbers}

Proper Fraction - A fraction $\frac{a}{b}$ for which $0 \leq|a|<b$.

Fraction Larger than One $-\mathrm{A}$ fraction where the numerator is at least as large as the denominator.

Note: Fractions larger than one are also called "improper fractions," but many schools are abandoning this term.

Mixed Number - A number that has a whole number component and a fraction component.

The Density Property of Rational Numbers - Let $r$ and $s$ be any two rational numbers, with $r<$ $s$. Then there is a rational number $t$ between $r$ and $s$; that is $r<t<s$.

In other words, if $\frac{a}{b}<\frac{c}{d}$, then there is a fraction $\frac{e}{f}$ for which $\frac{a}{b}<\frac{e}{f}<\frac{c}{d}$

\section{Addition and Subtraction of Fractions}

We will use diagrams, fraction circles, and Base 10 blocks to discuss what it means to add and subtract fractions. If, over the years, you have had trouble understanding fraction addition and subtraction, you may find that working with the diagrams and physical models will help you develop a better understanding.

\section{Methods for Adding and Subtracting Fractions}

You will notice that there are examples using different physical models that are meant to help you understand why addition and subtraction of fractions requires that the fractions be expressed with a common denominator. 
You should be able to add or subtract proper fractions, improper fractions and mixed numbers. You should also be able to illustrate examples of either process possibly using strip diagrams, polygon or circle diagrams.

Discussion: Examine the methods for adding and subtracting fractions. Which method do you find easier? Why? Why do you think students are taught to add and subtract fractions using the least common denominator?

\section{Arithmetic Methods for Adding Mixed Numbers}

Method \#1 -

Method \#2-

\section{Arithmetic Methods for Subtracting Mixed Numbers}

\section{Method \#1 -}

Method \#2-

The Role of Properties: Examine the methods above for adding and subtracting mixed numbers. Why do these methods work? Do properties of operations play a role in any of these methods?

\section{Multiplication and Division of Fractions}

The procedures for multiplying and dividing fractions and mixed numbers are reviewed. We also study models that illustrate the how and why these procedures work. Working with the diagrams and physical models will help you develop a better understanding. So, I recommend you should actually draw the diagrams so you understand how they might be useful in the case that you are teaching this topic. 


\section{Methods for Multiplying Fractions}

Method \#1-

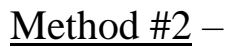

\section{Methods for Dividing Fractions}

The reciprocal: The reciprocal (the "multiplicative inverse") for a number $n$, is a number $1 / n$ such that

$n \times(1 / n)=1$. This is the inverse property of multiplication: the product of a number and its reciprocal equals 1 .

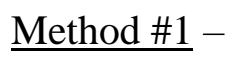

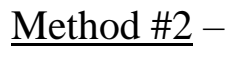

Method \#3-

Discussion: What is the value of $3 \frac{1}{2} \times 2$ ? Compute this using at least two different methods?

Consider how the distributive property can be used to quickly evaluate $3 \frac{1}{2} 2 \times 2$.

Using Properties: How can you use the distributive property to evaluate $81 / 4 \times 1 / 2$ ?

\section{Properties of Rational Numbers}

In the previous chapters we worked with the set of whole numbers, operating on whole numbers, and the properties of whole number operations. Then, we expanded our number system to include "negative numbers" and form the set of integers. The set of integers is $Z=\{\ldots-3,-2,-1$, $0,1,2,3 \ldots\}$. Now, we have further expanded our number system to include "fractional numbers" and form the set of rational numbers. The set of rational numbers is $\mathrm{Q}=\left\{\frac{p}{q} \mid p, q \in \mathbb{Z}, q \neq 0\right\}$. 
Can the properties of operations be extended to the set of rational numbers?

Properties of Addition - Let $a, b$, and $c$ be rational numbers.

\begin{tabular}{|l|l|l|l|}
\hline Property & Property Rule & Property Summarized & Example \\
\hline Commutative & & & \\
\hline Identity & & & \\
\hline Associative & & & \\
\hline Closure & & & \\
\hline Inverse & & & \\
\hline
\end{tabular}

Properties of Multiplication - Let $a, b$, and $c$ be rational numbers.

\begin{tabular}{|l|l|l|l|}
\hline Property Name & Property Rule & Property Summarized & Example \\
\hline Commutative & & & \\
\hline Identity & & & \\
\hline Associative & & & \\
\hline Closure & & & \\
\hline $\begin{array}{l}\text { Multiplication } \\
\text { by Zero } \\
\text { Property }\end{array}$ & & & \\
\hline $\begin{array}{l}\text { Distributive } \\
\text { Property }\end{array}$ & & & \\
\hline We introduce another Property & & \\
\hline Inverse & & & \\
\hline
\end{tabular}




\section{Decimals}

\section{Decimals and Real Numbers}

In previous chapters we studied, probably in greater depth than you ever thought possible, the whole numbers, integers and rational numbers. Now, we will explore what are commonly called decimal numbers like 17.568 or 0.34592 .

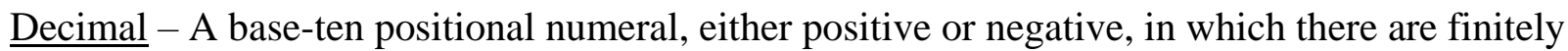
many digits extending to the left of a point called the decimal point that represents units, tens, hundreds, and so on, and a finite or infinite sequence of digits extending to the right of the decimal point that represents tenths, hundredths, thousandths, and so on.

\section{Expanded Form of Decimals}

Notice that you can express a number such as 3.854 in expanded form and that it is also possible to use manipulatives (blocks, mats, strips \& cubes) to represent such values.

\section{Fractions and Decimals}

You should note that any fraction can be written using decimal notation. Either the decimal has a finite number of digits and is described as a "terminating decimal" or the portion of the number to the right of the decimal point will contain a repeating pattern that never ends.

- Convert Fractions to Decimals

- Convert Decimals to Fractions

\section{Decimal Numbers on a Number Line}

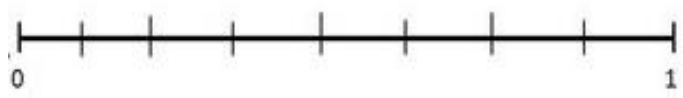

- Decimal place value

- Terminating and Repeating Decimals

- Ordering and Rounding Decimals

\section{The Set of Real Numbers}

Any number that you can plot on the number line is a real number. This includes all of the rational numbers and all of the irrational numbers. An irrational number is a number that cannot be written as a quotient of two integers such as $\pi$ and $e$.

\section{Adding and Subtracting Decimals}

To add or subtract decimal numbers, we must simply keep track of place value.

Compute each of the following:
a. $23.47+7.81$ 
b. $351.42-417.815$

\section{Multiplying Decimals}

You may remember the following algorithm for multiplying decimal numbers:

Multiplication Algorithm To multiply two decimals, do the following:

1. Multiply the two numbers as with integers.

To multiply $71.37 \times 6.125$, first multiply the two integers $7,137 \times 6,125=$ $43,714,125$.

2. Count the number of digits to the right of the decimal point in each factor in the product, add these numbers, and call their sum $t$.

There are 2 digits to the right of the decimal in 71.37 , and 3 to the right of the decimal in 6.125, so $t=2+3=5$.

3. Place the decimal point in the product that is obtained so that there are $t$ digits to the right of the decimal point.

Our final answer becomes $71.37 \times 6.125=437.14125$.

In order to understand why the multiplication algorithm works so well, let's explore why it works.

Using powers of ten:

$\underline{\text { Using improper fractions: }}$

Practice: Compute by hand.
a. $471.2 \times 2.3$
b. $36.34 \times 1.2$

\section{Dividing Decimals}

To divide decimals, students are frequently told to move the decimal place of both the dividend and the divisor the same number of places until both numbers are integers.

For example, when dividing $537.6 \div 2.56$, we would change the problem to: 
$537.60 \div 2.56 \quad 53760 \div 256$

Complete the division below.

Why does this division algorithm work?

Practice: Compute by hand.

$$
36.33 \div 1.2
$$

\section{Properties of Real Numbers}

In previous chapters we studied the whole numbers, integers and rational numbers. We started with the set of whole numbers: $W=\{0,1,2,3 \ldots\}$. Then, we expanded our number system to include whole numbers and their opposites to form the set of integer: $\mathbb{Z}=\{\ldots-3,-2,-1,0,1,2,3 \ldots\}$. Further, we expanded our number system to include "fractional numbers" and form the set of rational numbers: $\mathbb{Q}=\left\{\frac{p}{q} \mid p, q \in \mathbb{Z}, q \neq 0\right\}$.

Now, we expand our number system to include all of the rational numbers and all of the irrational numbers to form the set of real numbers $\mathbb{R}$.

Can the properties of operations be extended to the set of real numbers?

Properties of Addition - Let $a, b$, and $c$ be real numbers.

\begin{tabular}{|l|l|l|l|}
\hline Property & Property Rule & Property Summarized & Example \\
\hline Commutative & & & \\
\hline Identity & & & \\
\hline Associative & & & \\
\hline Closure & & & \\
\hline Inverse & & & \\
\hline
\end{tabular}


Properties of Multiplication - Let $a, b$, and $c$ be real numbers.

\begin{tabular}{|c|l|l|l|}
\hline Property Name & Property Rule & Property Summarized & Example \\
\hline Commutative & & & \\
\hline Identity & & & \\
\hline $\begin{array}{c}\text { Associative } \\
\text { Closure }\end{array}$ & & & \\
\hline $\begin{array}{c}\text { Multiplication by } \\
\text { Zero Property }\end{array}$ & & & \\
\hline $\begin{array}{c}\text { Distributive } \\
\text { Property }\end{array}$ & & & \\
\hline Inverse & & & \\
\hline
\end{tabular}




\section{APPENDIX G: DATA FROM AUDIO-TAPED INTERVIEWS}

\section{Ally and Betty}

Two MT1 PETs, Ally and Betty (pseudonyms), agreed to participate in audio-taped interviews. Ally and Betty participated in both the pre-teaching and post-teaching interviews. Both the pre-teaching and post-teaching interviews began with me, the interviewer, asking the participants to describe the distributive property. I started each interview by making the following statement:

I am going to say two words to you and, as soon as I say them, I want you to say something, or draw something, or do something - do the first thing that comes into your head after I say the words. Here are the words ... "distributive property. "Here are the words again: "distributive property." What comes into your mind?"

Both Ally and Betty gave appropriate responses to this prompt. Below in Figure G1 and Figure G2, their pre-teaching and post-teaching responses are presented.

Ally [Pre-Teaching]: To me, it means to take the mumber outside of the parentheses and distribute it to the mumbers or variables inside the parentheses.

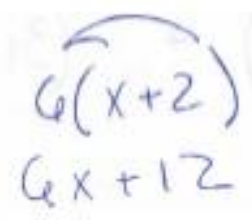

Ally [Post-Teaching]: So, basically you have to distribute when you are multiplying ... you have to distribute each term, um, to the other terms.

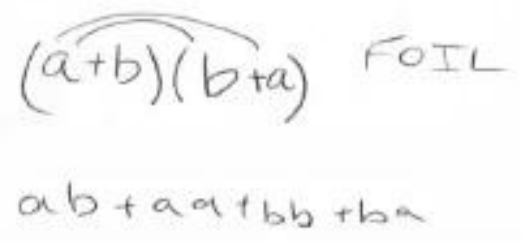

Figure G1. Ally's pre-teaching and post-teaching response to the first interview prompt. 
Betty [Pre-Teaching]: The first thing that comes to my mind is this thing that I was taught in algebra called FOIL. So, you have to, uh ... I don't know what to call them. But the FOIL acronym is First, Outer, Inner, Last, and you distribute to get, ... combine ... I would assume polynomial ... So, $x$ to $x$ is $x^{2}$, $x$ to 9 is $9 x, 9$ to $x$ is $9 x$, and the $9 \times 9$ is 81 . And then you can easily factor that out.

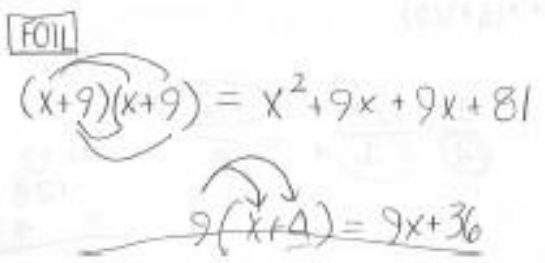

Betty [Post-Teaching]: So, distributive property the way that I think about it is that, if you have a term outside of the parentheses, we'll call it $a$, and you have two terms inside ... a mumerical expression inside, say $b+c$. The distributive property allows you to take $a$, and then multiply it within that mumerical expression to both terms.

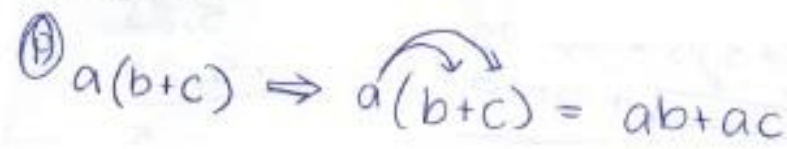

Figure G2. Betty's pre-teaching and post-teaching response to the first interview prompt.

All the interview prompts related to aspects of properties of real numbers. Three prompts dealt with aspects or use of the distributive property. One such interview prompt required participants to calculate the value of $315 \times 0.7+315 \times 0.3$ (pre-teaching) and $7 \times 97+7 \times 3$ (post-teaching) without the use of a calculator. Another interview prompt required participants to compute the value of $92 \times 5$ (pre-teaching) and $84 \times 5$ (post-teaching) and describe whether a number property was applied in the strategy used. An additional prompt on the post-teaching interview required participants to determine the value of $x$ that would make the following equation true: $5.25 x=70 \times 5.25+30 \times 5.25$.

Even though both Ally and Betty gave appropriate examples of the use of the distributive property during the pre-teaching interviews, neither of them recognized that the distributive property could applied to readily evaluate $315 \times 0.7+315 \times 0.3$. They both attempted to find the value of this expression by relying on order of operations. When Ally was asked about an alternate strategy for evaluating $315 \times 0.7+315 \times 0.3$, she was not able to articulate an alternate 
strategy that relied on order of operations. For Betty, the alternate strategy that she described was incorrect. Betty described a strategy that involved calculating the following sums, $0.7+0.3$ and $315+315$, and then determining the product of these two sums. Neither Ally nor Betty recognized that 315 could be factored from $315 \times 0.7+315 \times 0.3$ to yield $315 \times(0.7+0.3)=$ $315 \times 1=315$.

During the post-teaching interviews, both Ally and Betty also gave appropriate examples of the distributive property. One of the post-teaching interview prompts required Ally and Betty to evaluate $7 \times 97+7 \times 3$. Both Ally and Betty evaluated this expression using order of operations. Below, excerpts of Ally’s and Betty’s responses are given.

Ally: Um. So ... first I did $7 \times 3$ because that's easy. Um ... then I did $97 \times 7$... and then ... which got 679 and then I added 21 to get 700 .

Interviewer: Okay. Is there another way of doing it other than that?

Ally: Um ... I mean you have to like ... do it in that order.

Betty: So, I did $7 \times 3$ which is 21 ... that's a childhood fact. And then, I wasn't sure mentally what $97 \times 7$ is, so I used the standard algorithm for $97 \times 7$ to get 679 . Because we already had 21, we add it to 679 to get 700 as the final answer.

Interviewer: Uh, can you think of another way of doing it?

Betty: Do $7 \times 7$ which is 49 . Same process with $97 \times 3$. Same though. That's the same way.

Interviewer: Will that still lead you to 700 ?

Betty: It might. [Works it out on paper] No.

Interviewer: Which way are you more comfortable is appropriate? ...

Betty: The first way. 'Cause the second way you get 291, 291 plus 21 is not correct, at all. Too small.

Neither Ally nor Betty was able to describe an alternate way of evaluating $7 \times 97+7 \times 3$ that did not rely on order of operations. Even though both Ally and Betty presented appropriate examples of the distributive property during both the pre-teaching and post-teaching interviews, neither of them demonstrated strong understanding of how to apply the distributive property even after teaching had taken place. 
During the pre-teaching interviews, Ally and Betty were asked to compute the value of $92 \times 5$. The both used the traditional algorithm for multiplication that involves the concept of "carrying." When Ally was pressed to justify her strategy and to identify whether a number property was applied in her strategy, she responded as follows: "I guess it is the distributive property ... You are distributing the 5 to the 2 and the 9 , and then you are adding it." When Betty was pressed to justify why her strategy for evaluating $92 \times 5$ works, she responded, "here we go with that question [Betty laughs]. Uh, just works. I mean ... the validity of it I think it's just something that was not taught, and I don't know off the top of my head."

During the post-teaching interview, Ally and Betty were asked to compute the value of $84 \times 5$. Ally used the traditional algorithm for multiplication that involves the concept of "carrying." Ally's response to this prompt is presented in the excerpt below.

Ally: Um ... I took the 5 and multiplied it by 4. So, I got 20. Um ... so, I wrote down 0 and then carried the 2 . Then I did $80 \times 5$ and got 400 . And then, I still have the 20. So, I added $400+20$.

Interviewer: Why does your strategy work?

Ally: Um ... because it's like holding the place values ... and ... I'm basically like breaking it down into like $80 \times 5$ plus $4 \times 5$... which still ... yeah.

Interviewer: In your strategy, are you applying any property?

Ally: Um ... it's like the last one that we did. Except for this time, you kind of writing it out.

Interviewer: Can you specify which last one?

Ally: The one where it was $70 \times 5.25+30 \times 5.25$.

During the pre-teaching interview, Ally seemed to guess that the property she used in her algorithm was the distributive property. However, during the post-teaching interview, she could not name the property she used.

Betty also used the traditional algorithm for multiplication that involves the concept of "carrying." Betty's response to the prompt is presented in the excerpt below.

Betty: Standard algorithm ... [talks as she works it out] ... 5 times 4 is 20, carry the 2 to the next place value, 5 times is 40, plus 2, 42 zero ... 420. 
Interviewer: Okay. Can justify why that is a valid process?

Betty: Why that's a valid process?

Interviewer: Yeah. Why does that algorithm work?

Betty: The algorithm works because you kind of break down into its place value. So, you're multiplying your one's together. So, $4 \times 5$ you still get 20 .

Betty: And then you get 4 over here.

Interviewer: Anything else you would like to add beyond that?

Betty: You could do it another way. I found another way to do it.

Interviewer: What is the other way?

Betty: So, $80 \times 5$ is 400 because 8 times 5 is 40 and add the extra zero. And the $4 \times 5$ is 20. $400+20=420$.

Interviewer: What property are you relying on?

Betty: Associative property.

During the pre-teaching interview, Betty could not justify why her procedure for multiplying was valid. During the post-teaching interview, she was more successful at articulating why her strategy works. Even though she could articulate why her strategy works, she did not use the words "distributive property." Betty recognized that $84 \times 5=80 \times 5+4 \times 5=400+20=420$. However, when asked if any property was used, she replied, "associative property."

At the post-teaching stage, both Ally and Betty recognized that $84 \times 5=80 \times 5+4 \times 5$ but neither of them identified this as the use of the distributive property. During the post-teaching interview, the interviewees were asked to find the value of $x$ that would make the following equation true: $5.25 x=70 \times 5.25+30 \times 5.25$. In finding the value $x$, Ally relied on the concept of factoring but did not connect it to the distributive property. Ally's response and solution to this prompt are shown in Figure G3. 
Ally: Yeah ... since you are multiplying this by the same ... since you are multiplying 70 and 30 by the same number, you can combine them to make it 100-

Interviewer: You can combine which ones?

Ally: 70 and 30 .

Interviewer: Are you relying on any of the properties?

Ally: Yes. Um ... (long pause) ...

Interviewer: If you can't name it that's fine.

Ally: Transitive? I don't know.

$$
\begin{aligned}
& 5.25 x=70 \cdot 5.25+30 \times 5 \\
& 5.25 x= 70 \cdot 5.25+30 \cdot 5.25 \\
& 100 \times 5.25 \\
& 5.25 x= 525 \\
& 5.25 \\
& x=100
\end{aligned}
$$

Figure G3. Ally's response to $5.25 \mathrm{x}=70 \times 5.25+30 \times 5.25$ on the post-teaching interview.

Betty was not able to determine successfully the value of $x$. Her strategy for finding value of $x$ was incorrect and inappropriate for this prompt. Betty's response and solution to this prompt is given in Figure G4. The value of $x$ could be determined more readily by recognizing that the distributive property could be used to factor the right-hand side of the equation to obtain $5.25 x=$ $5.25 \times(70+30)$, which would mean $x=70+30=100$. 
Betty: We do have 5.25 on both sides, and if it was only one 5.25 on the right side, I would say divide each side by 5.25 and the do the math that follows. But there's two of them. Interviewer: Okay.

Betty: But because it's multiplication and addition you can rewrite it a little bit. Interviewer: How? Go ahead.
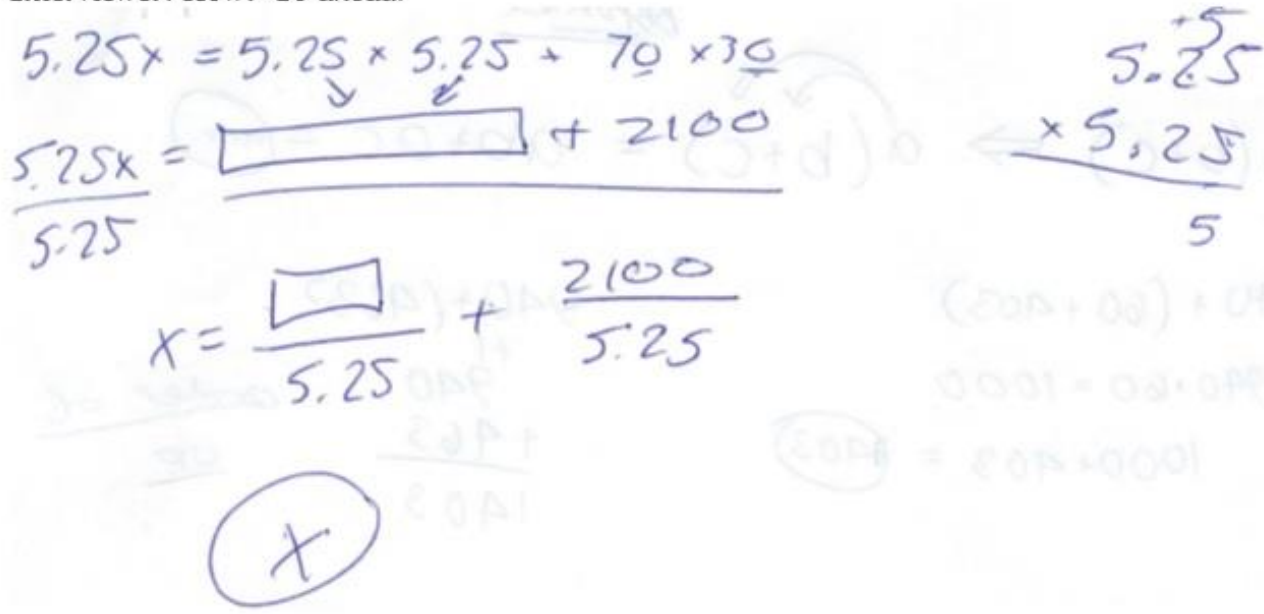

Figure G4. Betty's response to $5.25 \mathrm{x}=70 \times 5.25+30 \times 5.25$ on the post-teaching interview.

The preceding paragraphs dealt with Ally's and Betty's responses to prompts regarding their knowledge of, and capacity to apply, the distributive property to simplify or solve mathematical expressions. Other interview prompts covered other properties of real numbers including the associative, commutative, identity, and inverse properties.

During the pre-teaching interviews, interviewees were also given the following two prompts dealing with the associative property for addition and the associative property for multiplication:

- Without using a calculator, find the value of $482+(18+300)$.

- Without using a calculator, find the value of $4 \times(1 / 4 \times 128)$.

Both Ally and Betty relied on order of operations as a first strategy for calculating the value of $482+(18+300)$. An excerpt of Ally's response is presented in the exchange below.

Ally: So, I added what is in the parentheses first. I didn't do the distributive property because there was nothing to distribute outside of the parentheses. And then I added 482 and 318 to get 800. 
Interviewer: Can you think of a different way, a different process of finding the answer to the initial problem $482+(18+300)$ ?

Ally: Uhm ... [She draws on paper] you draw a number line.

Interviewer: Okay. What would that mean?

Ally: [She chuckles] It would be a really big number line.

Interviewer: Alright, it's okay.

Ally: But you could do ... you would like just, keep adding ... like 482 and then you would take the 18 and then you would add that ... and then add 300.

Interviewer: Are you able to tell me what property would make that valid?

$\ldots$

Ally: The property of addition

An excerpt of Betty's response is presented in the exchange below.

Betty: Okay. So, this takes me back. Uh, there was a principle I was taught called PEMDAS: Please Excuse My Dear Aunt Sally. It's pretty much the order of operations. Uh, and that tells you do anything in parentheses first. So, I'm just gonna copy down that problem so it is easier for me that way. So, with $18+300$ in the parentheses, that is 318. [As she works out the problem] And at that point you can just take the parenthesis off. So, you have $482+318$, and I cannot do mental math very well. So, I'm gonna do off to the side, like a stacked vertical algorithm I guess you could call it. And that's 800. So, your answer would be 800.

Interviewer: Can you think of a different way of doing it? [Student does not readily respond] Is there another way you could do it without relying on the process of PEMDAS?

Betty: I don't think so. I don't know any. If there are any, I don't know any.

The value of $482+(18+300)$ could be determined directly by applying the associative property for addition, which would mean $482+(18+300)=(482+18)+300=500+300=800$.

However, neither Ally nor Betty calculated the value of $482+(18+300)$ by using that property.

In calculating the value of $4 \times(1 / 4 \times 128)$, both Ally and Betty relied on order of operations as a first strategy. The value of $4 \times(1 / 4 \times 128)$ could also be determined more readily by applying the associative property for multiplication, which would mean $4 \times(1 / 4 \times 128)=(4 \times$ $1 / 4) \times 128=1 \times 128=128$. An excerpt of Ally's response to this prompt is presented in the exchange below.

Ally: Um ... So, first you have to do what is in the parentheses. So, I took 128 and divided by 4 , which is the same thing as multiplying it by $1 / 4 . \mathrm{Um}$... and then ... that the 
same ... [pause] you end up with 32 and then if you multiply by 4, you're just gonna go back to 128.

Interviewer: Can you think of a different way or a different process of doing it?

Ally: I mean you could just look at it and see that [referring to 4 and 1/4] these just cancels out.

Interviewer: Okay, and then?

Ally: You would just see 128 is the answer.

Interviewer: What mathematical property makes that legal?

Ally: Inverse operations

An excerpt of Betty's response to this prompt is presented in the exchange below.

Betty: So, again, I would jump right into PEMDAS. Now, I'm not gonna lie. That fraction had me for a second. [Works out problem on paper] So, you can easily figure out what is $1 / 4$ of 128 by doing long division. ... So, we know that this in the parentheses has to be 32 . So, we just to $4 \times 32 \ldots$ [works it out on paper] ... it's 128.

Interviewer: Is there a different way you could have done that?

Betty: I feel like there could be, if you were to figure out to do a least common denominator. But I am not sure, unless I work it out ...

Both Ally and Betty were asked to describe an alternative strategy, but Ally was the only one who recognized that the 4 and $1 / 4$ in $4 \times(1 / 4 \times 128)$ could be associated. However, she did not identify the associative property for multiplication by name. When asked to identify the property that allowed her to associate the 4 and $1 / 4$, she responded, "inverse operations." This is response is partially appropriate because 4 and $1 / 4$ are multiplicative inverses, and their product is 1 and $1 \times$ $128=128$.

During the post-teaching interviews, the following prompt dealing with the associative property for addition was presented: "without using a calculator, find the value of $940+(60+$ 403)." In calculating the value of $940+(60+403)$, both Ally and Betty relied on the associative property for addition as a first strategy. However, they both misidentified this property as the commutative property. An excerpt of Ally's response to this prompt is presented in the exchange below. 
Ally: Um ... So, I got rid of the parentheses because you don't ... [pauses] ... need them. Because even if you change the order it is still the same problem.

Interviewer: Okay.

Ally: So, then I added $940+60$ because that brings you up to a round even number of 1000. And then add the 403.

Interviewer: So, when you say you change the order, are you talking about the order of the numbers or the order of the parentheses? Or, both?

[No immediate response]

Interviewer: Or, I have confused you?

Ally: I didn't really change anything, it's just like in my head, like I just kind of took out the parentheses...

Interviewer: Are you applying any properties in your entire process?

Ally: Yes.

Interviewer: Can you name the property?

Ally: The ... [pauses] ... commutative.

Interviewer: Is this the only way to do it? Is there a different way of doing it?

Ally: Um, there is ... you could do it in different ways.

Interviewer: What would be a different for you to do it?

Ally: Um, you could do it with like base ten blocks or you could ... add ... more ... like you could add in a different order than I did. There's, just other things to do.

An excerpt of Betty's response to this prompt is presented in the exchange below.

Betty: So that one right there, $940+(60+403)$. Um ... because of the commutative

property, I would take the 60 out, add it to 940 to get 1000. And then add 403. So, you're left with 1403.

Interviewer: Okay. Now go ahead and write down what you-

Betty: Okay. [Proceeds to write]

Interviewer: Now, this is a second question. What if I ask if there's a different way to do it?

Betty: There is, but it would be a little longer.

Interviewer: Okay.

Betty: Do it as is. Keep 940 outside of the parentheses. 60 plus 403 would be 463. So, $940+463$... [as she works out on paper] would be ... 1403.

During the pre-teaching interviews, the first strategy of choice for both Ally and Betty was the

use of order of operations. Betty could not even articulate an alternative way for evaluating 482

$+(18+300)$ during the pre-teaching interview. However, in evaluating a similar expression 940

$+(60+403)$ during the post-teaching interview, Betty's first strategy of choice was associating

940 and 60. Even though both Ally and Betty relied on the associative property for addition as a 
first strategy for evaluating $940+(60+403)$, they both misidentified it as the commutative property.

During the post-teaching interview, Ally and Betty were presented with two prompts in which they could apply the associate property for multiplication.

- Without using a calculator, find the value of $(72 \times 5) \times 2$.

- Without using a calculator, find the value of $64 \times\left(\frac{1}{32} \times 120\right)$.

In calculating the value of $(72 \times 5) \times 2$, both Ally and Betty relied on the associative property for multiplication as a first strategy. However, as with the associative property for addition, both misidentified the associative property for multiplication as the commutative property. When calculating the value of $64 \times\left(\frac{1}{32} \times 120\right)$, only Ally associated 64 and $\frac{1}{32}$. An excerpt of Ally's response to this prompt is presented in the exchange below.

Interviewer: How did you find your answer?

Ally: Um, well ... $64 \times \frac{1}{32}$ is 2 . Because you essentially just taking 64 and dividing it by 32, which is 2. Um, and then you multiply that by 120, which is 240.

Interviewer: Okay. Assuming that what you are saying is right, what property would make that valid?

Ally: Um ... the commutative property of multiplication.

Even though Ally recognized that 64 and $\frac{1}{32}$ could be associated, she again misidentified the property used as the commutative property of multiplication. Betty, however, did not successfully calculate the value of $64 \times\left(\frac{1}{32} \times 120\right)$. An excerpt of Betty's response to this prompt is presented in the exchange below.

Betty: So, I know 32 goes into 64 twice.

Interviewer: Uh huh.

Betty: So, that what I'm like trying to figure it out ... [pauses] ...

Interviewer: Go ahead and solve it on paper. How you- 
Betty: I'm trying to like ... that's why I'm stuck like ... I know 32 times 2 [repeats] times 2 is 64 . But there is $\frac{1}{32}$. So, it's a portion of the whole which is 32 . Which would just be ... let me try it.

Uhm ... [pauses]. Made a little loophole till I figure out what to do with that fraction. You can always do $120 \times 64$. [Works it out on paper] ... wow, why did I do that? It'll be fine anyway ... 7680.

We have to do something with that 1 and 32 . Whatever it would be ... it'll have to be $7680 \times \frac{1}{32} \ldots$ we can always, maybe, multiply both sides by 32. Probably wrong, but I'm gonna take a shot. Just cancel out the denominator on that side. So, then it would be whatever $7680 \times 32$ is ... [inaudible speech as she works it out] ... really long-winded way to do it but gets the job done ... Sorry, my brain is like fried after that test.

Interviewer: It's alright.

Betty: Wow, why didn't I think of that? ... [Inaudible speech as she continues to work] ... really big number 245,760. It's probably way off. But that's best as I could do.

Interviewer: You've tried solving it one way. Is there a different way that maybe you could have approached it?

Betty: I feel like if I knew ... I just can't figure how if I could figure out what $\frac{1}{32}$ is in relationship to 64. If I knew what that answer was, then you could take that answer whatever it may be and then multiply by 120 to get your final solution.

Interviewer: Okay.

Betty: I just can't-

Interviewer: So, if you knew that ... are you applying any property?

Betty: The commutative property, pulls this out, pulls the $\frac{1}{32}$ out of the parentheses.

[Inaudible] do it by the 32 and you're still multiplying the whole thing by 120. So, that's the best that I can do on that one.

Even though Betty was unsuccessful in evaluating $64 \times\left(\frac{1}{32} \times 120\right)$, she recognized that 64 and $\frac{1}{32}$ could be associated. However, she was not sure about the appropriate operation for associating the 64 and $\frac{1}{32}$. When asked about the property that would allow her to associate 64 and $\frac{1}{32}$, she misidentified it as the commutative property.

The following prompts on the commutative property for addition were presented to Ally and Betty during the pre-teaching and post-teaching interviews.

- [Pre-teaching] Which value of $y$ would make the following equation true? $13+y=14+$ 
- [Post-teaching] Which value of $y$ would make the following equation true? $14+5=5+y$

These prompts were included to see whether participating interviewees would ascertain the $y$ by using the commutative property for addition. The excerpt below shows Ally's response to this prompt during the pre-teaching interview.

Ally: So, we subtract 13 from both sides. And, $y=14$.

Interviewer: Okay. Is there another way you could have done it? Suppose I had asked you to do it without writing anything down. How would you know what y is?

Ally: I mean you could just look at it and see that these [referring to the 13 on both sides of the equal sign] cancel out.

Interviewer: Okay. What mathematical property are we relying on? [Student does not Ally: 13 readily respond] You said something cancels out. What cancels out?

Interviewer: Are you relying on any mathematical property?

Ally: Yes.

Interviewer: Do you know the property?

Ally: Subtraction.

Ally's strategy of choice during the pre-teaching interview was determining the value of $y$ by isolating the variable. When asked about an alternate way of determining the value $y$, Ally referred to idea of "canceling" the common term on both sides of the equal sign. When pressed to state whether she used any properties, she replied, "Yes ... subtraction."

During the post-teaching interview, Ally's strategy of choice for determining the value of $y$ was the same as the one used during the pre-teaching interview. She determined the value of $y$ by isolating the variable. Her response is presented in the excerpt below.

Ally: Um ... we wanna ... to find the value of $y$. $y$ has to be the only thing on that side of the equation. To get rid of the 5, you have to subtract 5 to get 0 . Then y would be the only thing left. So then, you subtract 5 from both sides then you're left with 14 $=y$.

Interviewer: Alright. That is fine. Can you make an argument ... for why ... for why the value for $y$ is 14 without having to subtract 5 from both sides?

Ally: Um ... I mean you can just like ... see that there's the same value [referring to 5] on these sides and just kind of ignore them 
In both the pre-teaching and post-teaching interviews, Ally did not use the commutative property for addition to find the value for $y$.

The excerpt below shows Betty's response to the prompt on commutative property for addition during the pre-teaching interview.

Betty: So, if left side of $13+y$ is equal $14+13$, we know that 13 must be left alone because it's equal on both sides. But we have 14 on one side, and what you did on one side you have to do the other to make it equal. So, y has to be 14 in order to satisfy the fact that it's equal on both sides.

Interviewer: Okay. Um, do you ... is there property that allows you to say?

Betty: I would want to say that the commutative property, but I don't think that's right. But that the best shot I have.

To find the value of $y$, Betty argued that what is done one side of the equal sign must be done to the other side. She contended that if we start with 13 equals 13, then adding 14 to 13 on one side of the equal sign means that 14 must also be added to 13 on the other side of the equal sign.

When asked about the property being applied, she responded, "The commutative property, but I don't think that's right." During the post-teaching interview, Betty misidentified the property used as the associative property. However, when pressed to explain, she described a strategy in which she determined the value for $y$ by subtracting 5 from the sum of the left-hand side of the equal sign. Her response is presented in the excerpt below.

Betty: So, for both sides of the equation to be equal to each other, you must have y be equal to 14. Because 14 plus 5 ...

Interviewer: How do you know that $y$ is 14 ?

Betty: There's a property for ... I am pretty sure it is the associative property. It could be like throwing something out there hoping it sticks. But even if it's like, forgetting about properties for a second, if you have $14+5$ on this side is $19.5+y$ on that side. You have to have something to equal 19 on that side. So, you can always do $19-5=y$. You are left with $14=y$.

Interview prompts on the commutative property for multiplication were also presented to during the pre-teaching and post-teaching interviews. 
- [Pre-teaching] Which value of $y$ would make the following equation true? $5 y=4 \times 5$

- [Post-teaching] Which value of $y$ would make the following equation true? $4 \times 112=$ $112 y$

These prompts were included to see whether participating interviewees would ascertain the $y$ by using the commutative property for multiplication. Ally's strategy of choice during the preteaching interview was finding the value of $y$ by dividing both sides of the equal sign by 5 . She correctly identified $y=4$. When asked about an alternate way of determining the value $y$, Ally responded by cancelling the number 5 on both sides of the equal sign. When pressed on whether she used any properties, she replied, "division."

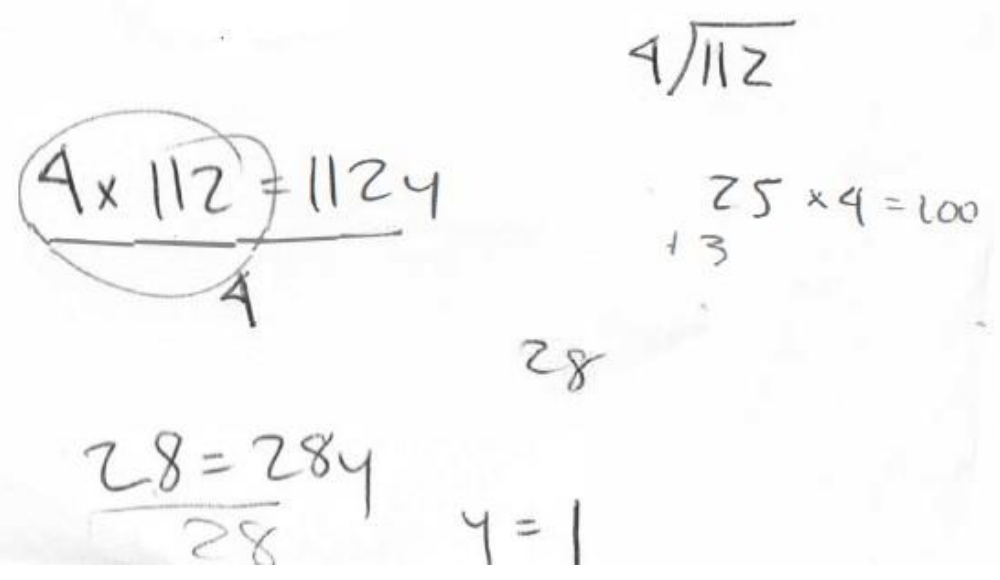

Figure G5. Ally's written response to $4 \times 112=112 \mathrm{y}$ on the post-teaching interview.

During the post-teaching interview, Ally did not correctly determine the value of $y$ would make the equation, $4 \times 112=112 y$, true. Her post-teaching interview response is presented in the excerpt below. Her written work is presented in Figure G5.

Ally: Okay. Um ... So, I re-wrote the problem and I'm getting rid of the 4. And I know 112 is divisible by 4 , so I don't have to do $4 \times 112$ first. So, I can straight across divide by 4 . And that's 28 . And I figured that out because $25 \times 4$ is 100 , and then that leaves 12. So, then $3 \times 4$ is $12.25+3$ is 28 . So, then $28=28 y$, and then just divide by 28 . And then $y=1$. 
Interviewer: Alright. Can you find your solution a different way? Is there a different way of finding your solution?

Ally: Um ... hmm ... I don't think so.

Betty's strategy of choice during the pre-teaching interview was determining the value for $y$ by dividing 5 from the product of the right-hand side of the equal sign. She correctly identified $y=4$. During the post-teaching interview, Betty referred to a strategy in which she divided both sides of the equal sign by 112 to determine the value for $y$. Her response is presented in the excerpt below.

Betty: That's pretty easy. Divide 112 by both sides. 112 is gone. So, 4 must equal y.

Interviewer: Okay. Can you make a case for why the value for $y$ is 4 without having to divide both sides by 112 ?

Betty: On both sides you are multiplying 112 by something. So, you are left with whatever 112 is being multiplied by. And again, to make it equal on both sides ... obviously the left side is $4 \times 112$. So, it's gotta be 4 on the right-hand side.

During both the pre- and post-teaching interviews, neither Ally nor Betty referred to the commutative property for multiplication when finding the value for $y$.

In both the pre-teaching and post-teaching interviews, the participating interviewees were asked to describe the meanings of the following terms: additive identity, additive inverse, multiplicative identity, and multiplicative inverse. The participating interviewees, Ally and Betty, did not demonstrate strong understanding of the meanings of this terminology during both the pre-teaching and post-teaching interviews.

During the pre-teaching interview, Ally gave following responses. When she was asked to describe the meaning of additive identity, she responded, "It means you can add numbers together." When she was asked to describe the meaning of multiplicative identity, she responded, "You can multiply numbers." When asked to describe the meaning of additive inverse, she first responded asking, "uh, the property of subtraction?" She then clarified, "it is the opposite of the 
additive property." When asked to describe the meaning of multiplicative inverse, she responded, "It is the opposite of the multiplicative property. So, the division property."

During the post-teaching interview, Ally gave following responses. When she was asked to describe the meaning of additive identity, she responded, "I don't know." When she was asked to describe the meaning of multiplicative identity, she responded that it means "there's a multiplication sign." When asked to describe the meaning of additive inverse, she responded, "When you do inverse operation you do the opposite of it. So, subtract." When asked to describe the meaning of multiplicative inverse, she gave the following response: "Multiplicative inverse $\ldots$ so like $\ldots$ this is the same as $7=7 x$. Instead of multiplying 7 and $x \ldots$ in order to solve for $x$ you have to do the inverse, which is division. So, you get $x=1 . "$

During the pre-teaching interview, Betty gave following responses. When she was asked to describe the meaning of additive identity, she responded, "I have never seen it before, but I would assume maybe it's a number in math problem that is being used in an addition problem." When she was asked to describe the meaning of multiplicative identity, she responded, "The two numbers in the multiplication problem would be your multiplicative identities." When asked to describe the meaning of multiplicative inverse, she responded, "We know inverse is when you do the opposite of something. So, I mean the only thing I can really think of that comes to mind when you think of the opposite of a multiplication problem is division." When asked to describe the meaning of additive inverse, she responded, "I can't."

During the pre-teaching interview, Betty stated she had not previously seen, or been taught the meaning of, any of these terms: additive identity, additive inverse, multiplicative identity, or multiplicative inverse. Her responses during the post-teaching interview are presented in the following excerpt. 
Interviewer: I'm gonna show you some terminology. I want you to state the terminology and tell me what it means to you.

Betty: Okay. The additive identity, I knew something like this would come back to bite me in the butt. Uhm, so ... the additive identity...

Interviewer: Write down ... you can try to write down to make sense of it.

Betty: I'm just blanking out on this one. I know the multiplicative identity of 1 and 0. Anything times 1 is 1 and anything 0 is 0 . But additive identity ... isn't it just ... I think it has something to do with negative numbers. So, if you have 4 and adding -4 to it ... because this is ... oh ... that's the inverse, that's the inverse. I don't know.

Interviewer: Are you sure?

Betty: Yeah. I am gonna pass on that one. That's the only one I am gonna probably like pass on. I'm like...

Interviewer: There's a card that fell. That's the next card I'm wanna ask you.

Betty: This one was the one that I was talking about ... [inaudible] ... multiplicative identity is pretty much anything times ... [slight pause] ... there's two identities for multiplicative stuff. So, it's any number, I'm gonna use 9 because we are on number 9, $9 \times 1$ would always equal itself. Anything times 1 equals itself. And then there's the other one for 0 , which is anything times 0 equals 0.

Interviewer: Okay ... what about this card: additive inverse? What does it mean?

Betty: So, the additive inverse is what I was trying to do on that one for additive identity. But ... so again if have 4 and you add -4 to it. Because -4 is the inverse of 4 they kind of cancel each other out as you would hear a young kid, or like myself, even say. So, then you are left with 0.

Interviewer: What about multiplicative inverse?

Betty: So, this one ... oh ... umm ... if you have a positive number, I'm just gonna use 4 again. I don't why I like 4's, multiply it by a negative number, the inverse. You still get a negative number. But you get what that value normally would be. So, the multiplicative inverse is if you multiply a positive by negative you get a negative, a positive by a positive you get a positive, a negative by a positive you get a positive. Because it is commutative, it doesn't matter whichever way you write it.

During the post-teaching interview, Betty appropriately described additive inverse and multiplicative identity. However, she was not able to describe the meaning of additive identity and gave an incorrect description of multiplicative inverse.

\section{Casey and Daisy}

One MT2 participant, Casey (pseudonym), and one MT3 participant, Daisy (pseudonym), agreed to participate in audio-taped interviews. Casey and Daisy only completed one interview each because there was no teaching intervention in the MT2 and MT3 courses. Each interview 
began with me, the interviewer, asking the participants to describe the distributive property. Only

Casey provided an appropriate description of the distributive property. Casey's response is

presented in the excerpt below. Casey's written work is provided in Figure G6.

Interviewer: So, I am going to say two words, and as soon as I say them, I want you to say or draw or do something. Do the first thing that comes to your head after I say these words on the piece of paper. The words are "distributive property." Here are the words again: "distributive property."

Casey: Um. I mean like I know [writes on piece of paper]

[As she writes] I don't even know if this is right. [After she finishes writing] I know the properties, but I don't know the names... I can't ... [pause]

Interviewer: You struggle with the names ... [Casey chuckles] that's fine

Casey: I know transitive, cause uh ... [chuckles, pauses]

Interviewer: That's okay

Casey: I like the word.

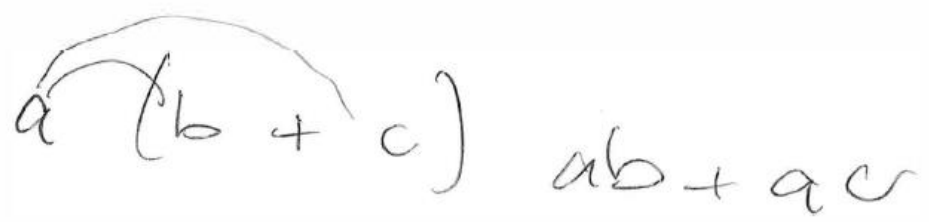

Figure G6. Casey's written description of the distributive property.

Even though Casey provided an appropriate description of the distributive property, she stated that she was not sure whether she was right. Daisy did not provide an appropriate description of the distributive property. She, instead, described the traditional procedures for adding multi-digit numbers. Daisy's response is presented in the excerpt below. Daisy's written work is provided in Figure G7.

Interviewer: Alright, I am going to say two words, and as soon as I say them, I want you to say something or draw something or do something. Do the first thing that

Daisy: Okay. comes to your head after I say these words.

Interviewer: The words are "distributive property." Here are the words again, "distributive property."

Daisy: So, if I was to do something like ... [begins to write] ... That is ridiculous. I know what I meant in my head.

Interviewer: It's okay. 
Daisy: Wait...

Interviewer: Can you also say in words what you did?

Daisy: So, I just... I wrote down 19 and 12 ... and added the two numbers together so I added $9+2$...to get 11 and then carried the 1 and added the 3 once to get 31 .

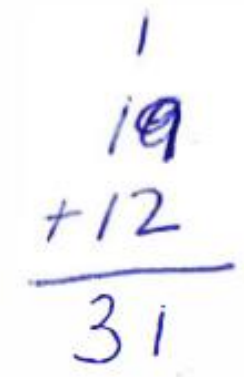

Figure G7. Daisy's written description of the distributive property.

There were three other interview prompts that dealt with aspects of, or use of, the distributive property.

- Calculate the value of $315 \times 0.7+315 \times 0.3$ without the use of a calculator.

- Determine the value of $x$ that would make the following equation true: $5.25 x=70 \times$ $5.25+30 \times 5.25$

- Compute the value of $92 \times 5$ and describe whether a number property is applied in your strategy.

Even though Casey had previously provided an appropriate description of the distributive property, she did not recognize that the property could be used to readily evaluate $315 \times 0.7+$ $315 \times 0.3$. She inappropriately attempted to find the value of $315 \times 0.7+315 \times 0.3$ by first adding like terms and then determining the product. When asked about an alternate way of evaluating $315 \times 0.7+315 \times 0.3$, Casey attempted to multiply $315 \times 0.7$ but was unsuccessful because she was not comfortable working with decimals. Casey's written work for this prompt is provided in Figure G8. 


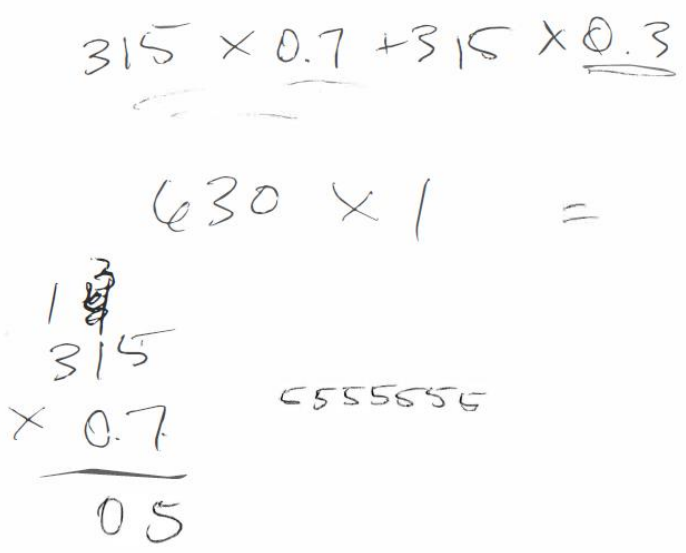

Figure G8. Casey's written work for $315 \times 0.7+315 \times 0.3$.

Daisy relied on order of operations to find the value of $315 \times 0.7+315 \times 0.3$. When asked about an alternate way of evaluating this expression, she replied, "I'm sure there is, but I don't know what that will be." Daisy's written work for this prompt is provided in Figure G9. An excerpt of Daisy's verbal response is presented below.

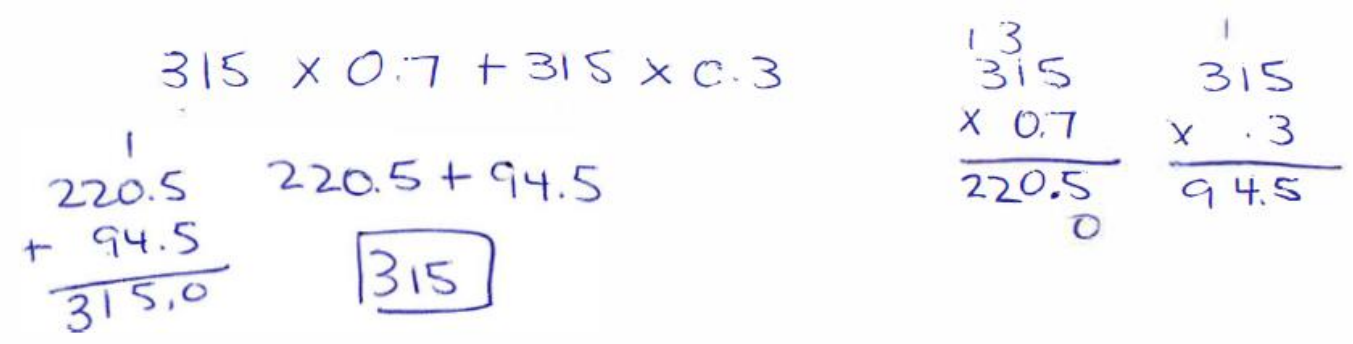

Figure G9. Daisy's written work for $315 \times 0.7+315 \times 0.3$.

Daisy: I wrote out the problem. I multiplied 315 by .7 to get 220.5, and I multiplied 315 by .3 to get 94.5 , and added the two together to get 315 .

Interviewer: So, why did you do that?

Daisy: I just went from either side ... so, to multiply the numbers out first, and then add them together.

Interviewer: You multiplied first because of?

Daisy: I know that this is not the right answer, but "Please Excuse My Dear Aunt Sally" Interviewer: There is nothing wrong with using-

Daisy: I don't know what the actual terminology is for, but I know that's why I'm doing it.

Interviewer: Okay. Is there any other way you that you could have done it without relying on that ... a different strategy from the one you used? 
Daisy: Um, I'm sure there is, but I don't know what that will be.

In determining the value of $x$ in the following equation, $5.25 x=70 \times 5.25+30 \times 5.25$, Casey's first strategy of choice was dividing all terms in the equation by 5.25 and then simplifying the right-hand side of the equation by finding the sum of 70 and 30 . When asked about an alternate strategy for finding the value of $x$, Casey factored 5.25 on the right-hand side yielding $5.25 x=5.25 \times(70+30)$. When asked if she used any properties, she responded, "equality ... is that the property?" Casey was not able connect the concept of factoring to the distributive property. Her written work for this prompt is presented in Figure G10.

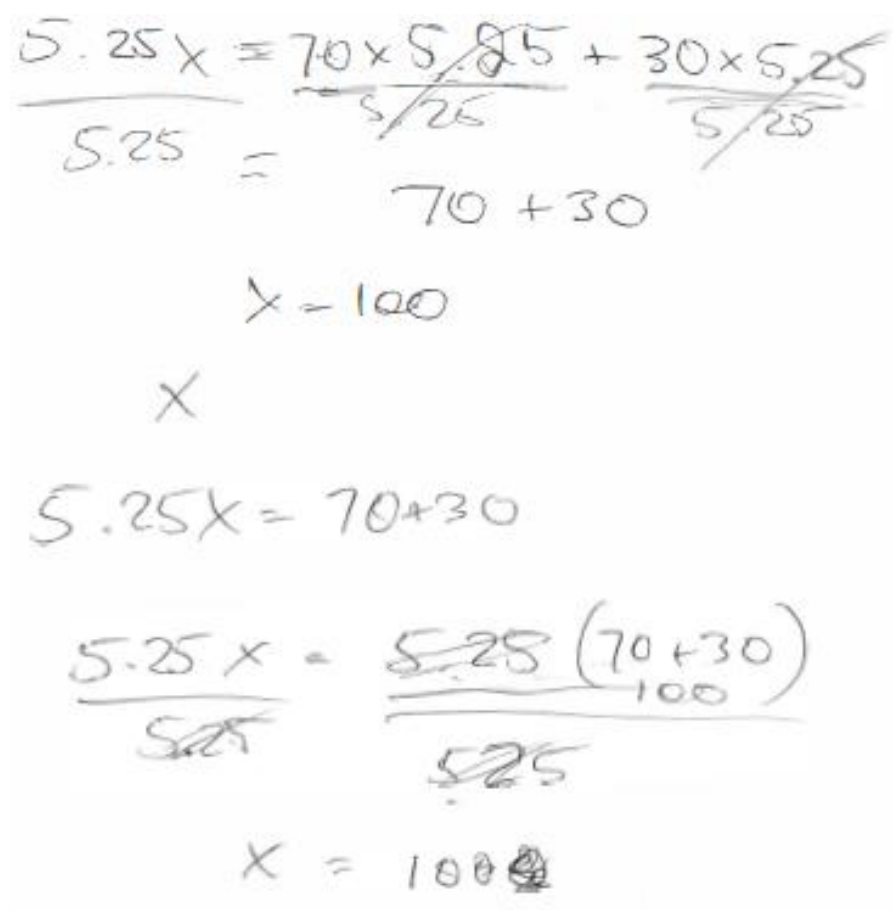

Figure G10. Casey's written work for $5.25 \mathrm{x}=5.25 \times(70+30)$.

In determining the value of $x$ in the following equation, $5.25 x=70 \times 5.25+30 \times 5.25$, Daisy's first strategy of choice was relying on order of operations to simplify the right-hand side of the equation and then dividing by 5.25 on both sides of the equal sign. When asked to describe an alternative strategy, she divided all terms in the equation by 5.25 and then simplified the right- 
hand side of the equation by finding the sum of 70 and 30 . She did not mention anything relating to the distributive property. Daisy's written work for this prompt is presented in Figure G11.

$$
\begin{aligned}
& 5.25 x=70 \times 5.25+30 \times 5.25 \times 19.2 .25 \\
& 5.25 x=367.50+157.50 \\
& \frac{5.25}{5.25} \times \frac{525}{5.25} \\
& 000 \\
& 367.50 \\
& 5.2^{1} 5 \\
& \begin{array}{r}
30 \\
\hline 000
\end{array} \\
& 157.50 \\
& \begin{array}{l}
11 \\
367.50
\end{array} \\
& 157.50 \\
& 525.00 \\
& 5 . 2 5 \longdiv { 5 2 5 } \\
& 5.25 x=70(5.25)+30(5.25) \\
& 5.25 \quad 5.25 \\
& x=70+30 \\
& x=100
\end{aligned}
$$

Figure G11.Daisy's written work for $5.25 \mathrm{x}=5.25 \times(70+30)$.

During the interviews, Casey and Daisy were asked to compute the value of $92 \times 5$ and to describe whether a number property was applied in their strategies. Casey's response is presented in the excerpt below.

Interviewer: Alright, tell me what you did.

Casey: Um ... Long multiplication ... [laughs]

Interviewer: What is long multiplication? 
Casey: So, I learned how to multiply by putting the numbers vertical. And then I do $5 \times 2$, which is 10, and then I carry it over ... the zero, $5 \times 9$ is 45, and then I add 1 to it. And put 46.

Interviewer: Okay. What makes this process valid? Why should I trust that this process works?

Casey: [Laughs] ...um, let me think about that for a second ...

Interviewer: Yeah, why does that work? [No response from Casey] Does it rely on any property? And why did you have to carry 1? Why couldn't you just write 10? What would have happened if you had written, like $2 \times 5$ is 10 ? What would happen if you put 10 down?

Casey: So ... I couldn't put 10 because I haven't finished the hundreds place or the... the... second one. So, I would have to add that to that, and [inaudible] 0. If I put 10 there, I would end up getting 460.
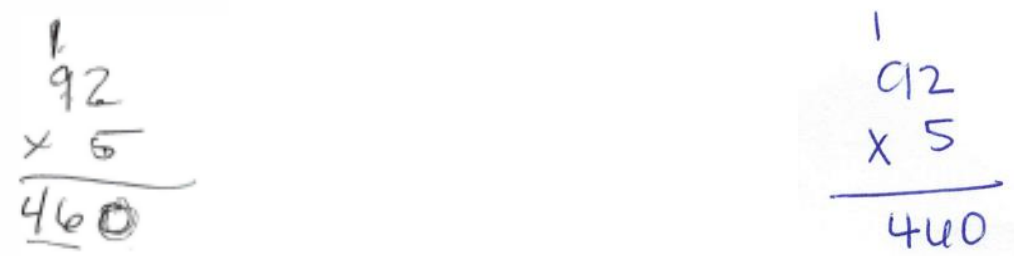

Figure G12. Casey's written work for $92 \times 5$. Figure G13. Daisy's written work for $92 \times 5$.

Daisy's verbal response is presented in the excerpt below. When Casey and Daisy were asked to describe why their strategies for multiplication were valid, neither was able to articulate the reasoning in their strategies. Neither Casey nor Daisy connected their strategies for multiplication to the distributive property.

Interviewer: Okay, tell me what you did.

Daisy: So, I wrote $92 \times 5$. I multiply $5 \times 2$, brought down the 0 and carried the 1. Did 9 $\times 5$ which is 45 , added the 1 and got 460 .

Interviewer: So, why does this process work? Why should I trust this process works?

Daisy: I mean, I guess I will say because it's ... the numbers are there, if you know the math ... [laughs]

Interviewer: Why do you have to carry 1?

Daisy: Um...

Interviewer: When you did $5 \times 2$, why didn't you just write 10 down?

Daisy: Because it's the one's place, tens place, and hundreds place. So, there is tens and ones, and the number 10. So, I had to write the ones in the one place and carry the other number.

Interviewer: And how come you started with 2 times 5? Why not start with the 9 and 5?

Daisy: Um, it's the order that you multiply in.

Interviewer: Can ... if I change the order, will I be wrong? 
Daisy: Yes.

Interviewer: Okay. Does this strategy apply or rely on any number property?

Daisy: Like you have to know your multiplication facts, like your times tables.

In the preceding paragraphs, I presented Casey's and Daisy's responses to the prompts assessing their knowledge of, and capacity to apply, the distributive property to simplify or solve mathematical expressions. The following two interview prompts dealt with the associative property for addition and the associative property for multiplication:

- Without using a calculator, find the value of $482+(18+300)$.

- Without using a calculator, find the value of $4 \times(1 / 4 \times 128)$.

Both Casey and Daisy relied on order of operations as a first strategy for calculating the value of $482+(18+300)$. However, Casey's first attempt led her to conclude incorrectly that the value of $482+(18+300)$ is 600 .

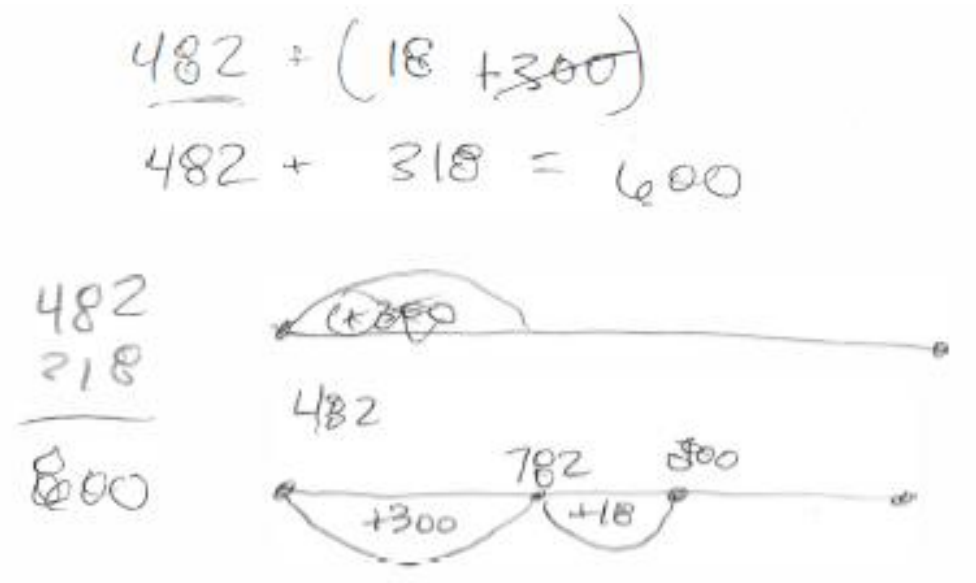

Figure G14. Casey's written work for $482+(18+300)$.

When asked to describe an alternate strategy, she drew a number line in which she started with 482, added 300, and then added 18. After using this strategy, Casey realized that her initial answer was incorrect. So, she corrected her answer to 800. When she was asked whether she used any properties, she replied with a question, “the addition property?" Casey's written response is shown in Figure G14. 
Daisy evaluated $482+(18+300)$ by first computing $18+300$ and then adding this sum to 482. When she was asked to explain, she responded, "You do the 'Please Excuse My Dear Aunty Sally." When asked to describe alternative strategy, Daisy responded, “Add 300 to 482 to get 782, and then add the 18." When asked whether she used any properties, she replied, "I don't know." Daisy's written response to $482+(18+300)$ is shown in Figure G15.

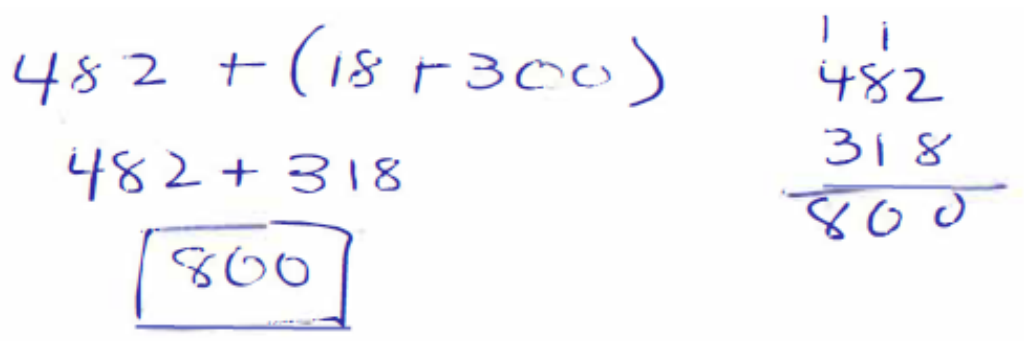

Figure G15. Daisy's written work for $482+(18+300)$.

When calculating the value of $4 \times(1 / 4 \times 128)$, both Casey and Daisy relied on order of operations as a first strategy. Casey initially emphasized that she needed to first calculate what was in the parenthesis. When asked to describe an alternate strategy, she responded, "If I could just get the thought out of my head that I have to acknowledge the parentheses. Um, I would know that $4 \times 1 / 4$ is 1 , and then that would be $1 \times 128$." When asked to about the property employed, she responded with a question, "multiplication?" Casey's written work for $4 \times(1 / 4 \times$ 128) is shown in Figure G16.

Daisy evaluated $4 \times(1 / 4 \times 128)$ by first computing $1 / 4 \times 128$. She changed $1 / 4$ into a decimal and computed $0.25 \times 128$. Daisy stated that she changed the fraction to its decimal equivalent because she was more comfortable with decimals than fractions. When asked about using an alternate strategy to evaluate $4 \times(1 / 4 \times 128)$, she did not respond except with some laughter. Her written work for $4 \times(1 / 4 \times 128)$ is reproduced in Figure G17. 

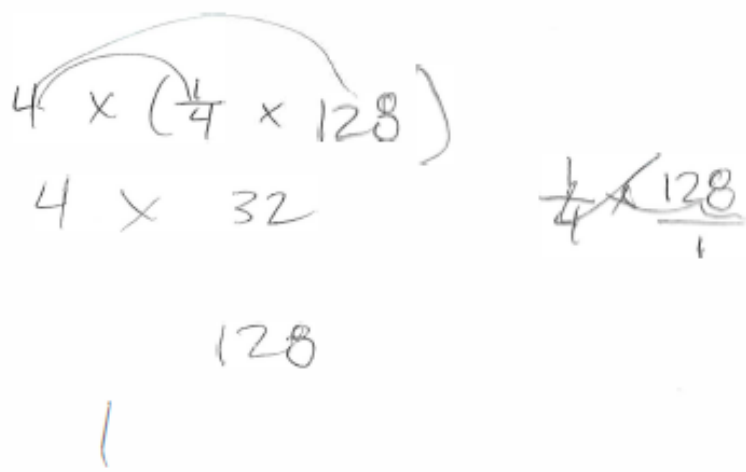

Figure G16. Casey's written work for $4 \times(1 / 4 \times 128)$.

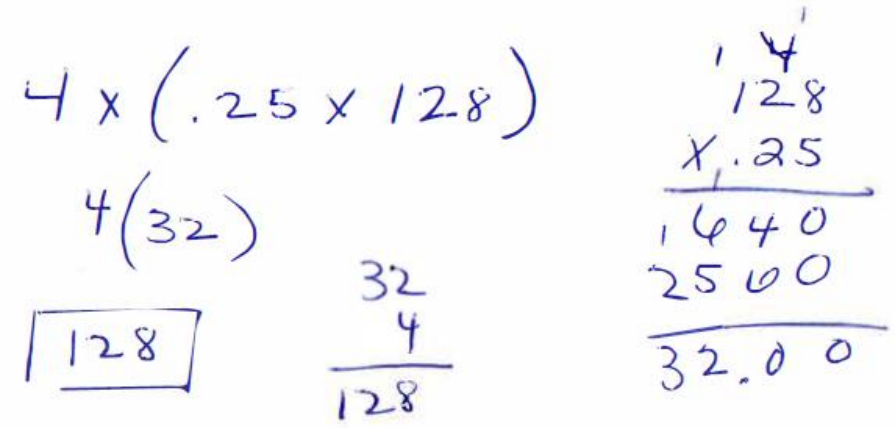

Figure G17. Daisy's written work for $4 \times(1 / 4 \times 128)$.

The following interview prompts dealt with the commutative property for addition: Without using a calculator, find the value of $13+y=14+13$. This prompt was included to see whether participating interviewees would ascertain the $y$ by using the commutative property for addition. Both Casey and Daisy correctly stated that the value for $y=14$ without giving an explanation. When Daisy was asked to explain how she obtained $y=14$, she responded, "Because you will subtract 13 from both sides. You're left with $y=14$." However, when I asked to think of an alternate way of determining $y=14$, she could not come up with one.

When I asked Casey to explain how she arrived at $y=14$, she responded, "I would subtract 13 from the sides, and this would cancel out, so $y$ would be 14." Her strategy relied on 
isolating the variable. The excerpt below shows the exchange between me, the interviewer, and Casey after I had asked her for alternate way of finding the value of $y$.

Interviewer: Okay. Without having to try and isolate y, can you make a case why y is 14 ? Without having to try to isolate $y$, make a case for why y is 14 ?

Casey: [inaudible]

Interviewer: Can you make a case? Can you make for why y is 14 without having to-?

Casey: Reflexive

Interviewer: Reflexive, what is reflexive property?

Casey: So, it's ... um ... $a=a$, or $a+b=b+a$.

Interviewer: So, which one are you relying on? This one or this one?

Casey: I guess this one [referring to $a+b=b+a$ ] for that one [referring to $13+y=14$ $+13]$.

Interviewer: But they're not the same thing [referring to $a=a$, and $a+b=b+a$ ]?

Casey: They're not. I know. I still get confused with that.

When asked about an alternate way for why $y=14$, Casey referred to the "reflexive property."

When pressed about what this property states, she was unsure of whether it described $a=a$, or $a$ $+b=b+a$. She did, however, clarify that she knew $y=14$ because $a+b=b+a$.

As with Ally's and Betty's pre-teaching and post-teaching interviews, Casey and Daisy were also asked to describe the meanings of the following terms: additive identity, additive inverse, multiplicative identity, and multiplicative inverse. Casey and Daisy had practically no knowledge of any of the meanings of this terminology.

When I asked Casey to tell me the meaning of additive identity, she replied, "The additive identity is something ... I know that I have learned it ... uh ... It has something to do with addition, and it identified something." When I asked her to tell me the meaning of multiplicative identity, she acknowledged she did not know how to respond. When I asked her for the meaning of additive inverse, she wrote $a+b=b+a$, and asked: "Is that what this is?" Lastly, when I asked her for the meaning of multiplicative inverse, she responded, "Multiplicative inverse ... Does it have something to do with fractions?" 
When I asked Daisy to tell me the meaning of additive identity, she responded, "I don't know actually know what it means, but what it makes me think of is, like, knowing how to add like numbers together." When I asked her to tell me the meaning of multiplicative identity, she replied, "Again, for me, it will mean knowing how to multiply, like your multiplication facts. But I don't know the answer." When I asked her for the meaning of additive inverse, she gave an analogous response: “Is that like subtraction? It's what it makes me think of, but I don't think that's correct." Lastly, when I asked her for the meaning of multiplicative inverse, she again responded in an analogous manner: "Again, it makes me think of dividing instead of multiplying." 